\title{
Synthesis of Vitisins A \& D Enabled by a Persistent Radical Equilibrium
}

Kevin J. Romero, ${ }^{1}$ Mitchell H. Keylor, ${ }^{1}$ Markus Griesser, ${ }^{2}$ Xu Zhu, ${ }^{1}$ Ethan J. Strobel, ${ }^{1}$ Derek A. Pratt ${ }^{2, *}$ and Corey R. J. Stephenson ${ }^{1, *}$

${ }^{I}$ Department of Chemistry, University of Michigan, 930 North University Avenue, Ann Arbor, MI 48109, USA

${ }^{2}$ Department of Chemistry and Biomolecular Sciences, University of Ottawa, Ottawa, Ontario, Canada KIN 6 N5

\section{Supporting Information}

\section{Table of Contents}

1. General Procedures $\quad$ S2

2. Reaction Materials $\quad$ S2

3. Product Analysis $r$

4. Figure S1. Biosynthetic Hypothesis for Resveratrol Oligomerization $\quad$ S3

5. Prior Biomimetic C3-C8' dimerization efforts $\quad$ S3

a. Table S1. Prior examples of oxidative conversion of resveratrol (S1) to $\delta$-viniferin $(\mathbf{1 1 A}) \quad$ S3

b. Figure S2. Sako and co-worker's semisynthesis of vitisin B (5A) from isolated (+)-E-viniferin $\quad$ S4

6. Figure S3. Van't Hoff Analysis of the 8A-9A-10A equilibrium $\quad$ S4

7. Experimental Procedures and Characterization Data $\quad$ S5

$\begin{array}{lll}\text { a. } & \delta \text {-viniferin core } 11 & \text { S5 }\end{array}$

b. Figure S4. Crossover experiment between $\mathbf{8 A}$ and S5 $\quad$ S6

c. Figure S5. Preparation of acetophenone 14A and 14B and alternate preparation of $16 \quad$ S6

$\begin{array}{ll}\text { d. Compound S10 } & \text { S7 }\end{array}$

e. Compound $\mathbf{S 1 2}$ S8

f. Compound S11 S9

g. Compound 14A S10

h. Compound S13 $\quad$ S11

i. Compound S14 $\quad$ S12

j. Compound S15 $\quad$ S13

k. Compound 14B S14

1. Compound 15A $\quad$ S15

m. Compound S19 $\quad$ S17

n. Compound S17 $\quad$ S19

o. Compound S18 $\quad$ S21

p. Compound $\mathbf{1 6} \quad$ S23

q. Compound 15B $\quad$ S24

r. Compound $\mathbf{S 2 1} \quad$ S26

$\begin{array}{lr}\text { s. Compound 13B } & \text { S27 }\end{array}$

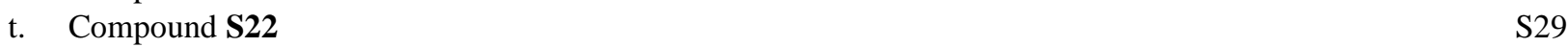

u. Compound $\mathbf{1 6} \quad$ S31

v. Compound $\mathbf{1 7} \quad$ S33

w. Compound 12A $\quad$ S35

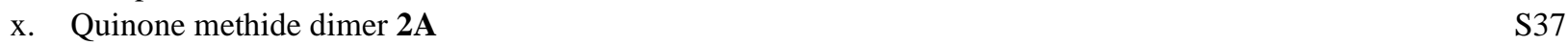

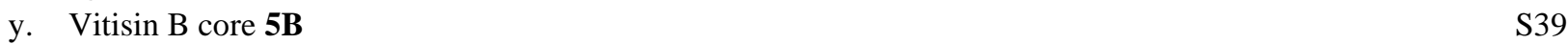

Z. Compounds 19/20 S42

aa. Crossover experiment with $\mathbf{2 A}$ and $\mathbf{2 B} \quad$ S44

bb. Vitisin A (6) $\quad$ S45

cc. Vitisin D (7) $\quad$ S52

dd. Compounds 20/21 $\quad$ S59

i. Table S6 - Attempts at C5c-desilylation $\quad$ S61

8. Computational Data $r$ S62

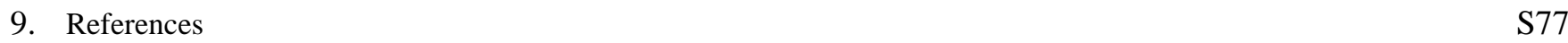




\section{General Procedures:}

Unless specifically noted otherwise, all glassware was flame-dried under vacuum ( 0.5 Torr) and cooled under inert atmosphere $\left(\mathrm{N}_{2}\right.$ or Ar) prior to use. Each reaction container was charged with a Teflon/PTFE-coated magnetic stir bar and sealed with a rubber septum to maintain a positive pressure of inert atmosphere $\left(\mathrm{N}_{2}\right.$ or Ar). Reagents sensitive to the atmosphere were transferred via syringe or cannula as necessary. Reactions that required microwave irradiation were conducted in a Biotage Initiator+ Microwave Synthesizer. Reaction conversion was evaluated using analytical thin-layer chromatography (TLC) using Merck silica gel 60 F254 TLC plates. TLC plates were visualized under a dual short wave/long wave UV lamp and/or stained using solutions of $p$-anisaldehyde or potassium permanganate or ceric ammonium molybdate. Stained plates were developed over a heat gun as needed. Either sodium sulfate or magnesium sulfate were utilized to exclude water from worked up reactions, and the solvent was removed on Büchi rotary evaporators and/or a Welch vacuum pump. Reactions were purified via flash column chromatography either with Biotage SNAP Ultra chromatography cartidges using a Biotage Isolera automated purification system or manually using 230-400 mesh silica gel. Final purification of natural products was achieved using reverse phase preparative HPLC on an Agilent 1290 Infinity II Preparative LC/MSD System. All electrochemical experiments were acquired using either a $\mathrm{CH} 1620 \mathrm{E}$ electrochemical analyzer (from $\mathrm{CH}$ Instruments) or a uSTAT4000 4-Channel Potentiostat/Galvanostat (from Metrohm USA). Bulk electrolysis experiments were performed on discovery scale in open $10-\mathrm{mL}$ vials and in a beaker of the appropriate size $(15-40 \mathrm{~mL})$ for the subsequent scale-up experiments. These reactions used RVC panels (reticulated vitreous carbon, 100 ppi, 0.25-inch thickness, 3\% relative density, from McMaster Carr) as the working or counter/auxiliary electrodes and a $\mathrm{Ag} / \mathrm{AgCl}(3 \mathrm{M}$ $\mathrm{KCl})$ reference electrode.

\section{Reaction Materials:}

Commercially available reagents were used without further purification unless specified. Organic solvents (acetonitrile, dichloromethane, diethyl ether, dimethylformamide, dimethyl sulfoxide, methanol, tetrahydrofuran, and toluene) and amine bases (triethylamine, pyridine, N,N-diisopropylethylamine, and diisopropylamine) were purified prior to use with a Phoenix Solvent Drying System from JC-Meyer Solvent Systems and PureSolv Micro amine drying columns from Innovative Technology, respectively, and kept under a pressure of argon. Solutions of organolithium reagents and Grignard reagents were purchased from Acros Organics and titrated prior to use.

\section{Product Analysis:}

Product names were obtained using ChemDraw Professional 16.0 from Perkin Elmer. For racemic compounds, the name corresponds to the depicted structure. Nuclear magnetic resonance (NMR) spectra were obtained using an internal deuterium lock on Varian Inova 500 or Varian VNMR 500 and 700 spectrometers. For ${ }^{1} \mathrm{H}$ spectra, chemical shifts were referenced to the center line of the residual solvent signal $\left(\mathrm{CDCl}_{3}: \delta 7.26\right.$; acetone- $d_{6}: \delta 2.05$; methanol- $\left.d_{4}: \delta 3.31\right)$ and are reported in parts per million (ppm). Signal multiplicity is reported as follows: $(\mathrm{br}=$ broad, $\mathrm{s}=$ singlet, $\mathrm{d}=$ doublet, $\mathrm{t}=$ triplet, $\mathrm{dd}=$ doublet of doublets, $\mathrm{ddd}=$ doublet of doublet of doublets, $\mathrm{m}=$ multiplet $)$, and the associated coupling constants are given in Hertz. For ${ }^{13} \mathrm{C}$ spectra, experiments were completely heterodecoupled (broadband) and chemical shifts are reported as ppm using the center line of the solvent signal as reference $\left(\mathrm{CDCl}_{3}: \delta 77.16\right.$; acetone- $d_{6}: \delta 29.96$; methanol- $\left.d_{4}: \delta 49.00\right)$. The following resveratrol numbering scheme was used for the assignment of ${ }^{1} \mathrm{H}$ and ${ }^{13} \mathrm{C}$ NMR signals. High-resolution mass spectra (HRMS) were acquired using a Micromass AutoSpec Ultima Magnetic Sector mass spectrometer using electrospray ionization

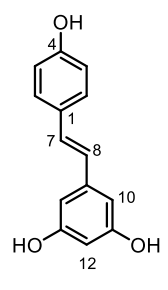
(ESI), positive ion mode. We thank James Windak and Paul Lennon at the University of Michigan, Department of Chemistry Instrumentation Facility for conducting the HRMS experiments. Infrared spectra were acquired using a Perkin-Elmer Spectrum BX FT-IR spectrophotometer using an ATR mount with a ZnSe crystal. UV-Vis spectra were recorded on an Agilent Cary $100 \mathrm{UV}$-Vis spectrophotometer equipped with a 6x6 thermostated multi-cell holder and a Cary temperature controller unit. Electron paramagnetic resonance (EPR) spectra were recorded on a Bruker EMXplus (X-band) equipped with an ER $4119 \mathrm{HS}$ cavity in a temperature range from 10 to $50{ }^{\circ} \mathrm{C}$. Samples were typically $50 \mu \mathrm{M}$ in benzene and the radical concentration was determined using the quantitative EPR package of the Bruker Xenon software. 


\section{Biosynthetic Hypothesis for Resveratrol Oligomerization:}

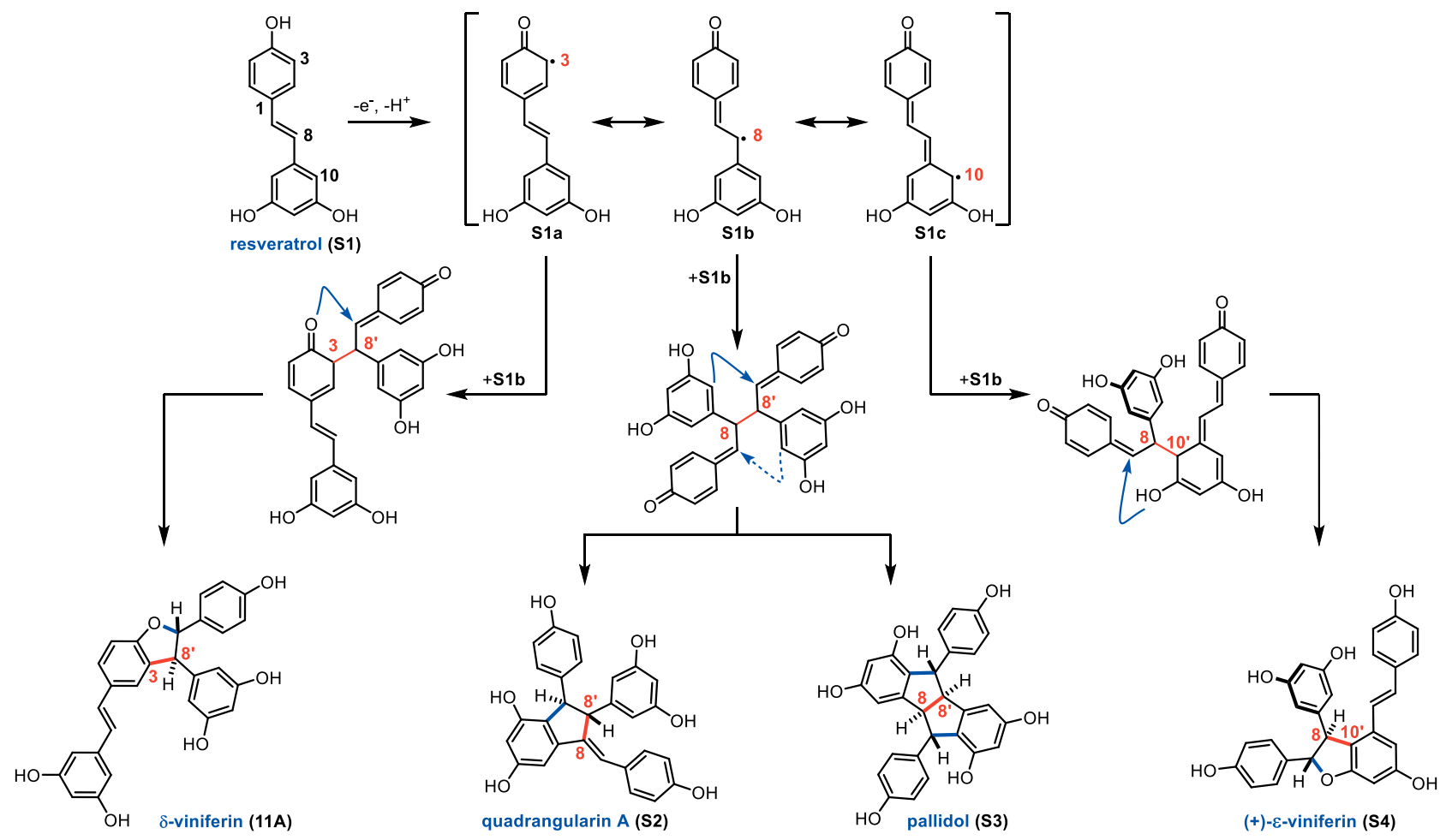

Figure S1. Resveratrol (S1) oligomerization is proposed to occur via radical intermediates S1a-c to afford the C3-C8', $\mathrm{C} 8-\mathrm{C} 8$ ', and $\mathrm{C} 8-\mathrm{C} 10^{\prime}$ bond connections exhibited across the natural product class.

\section{Prior Biomimetic C3-C8' dimerization efforts:}

Table S1. Prior examples of oxidative conversion of resveratrol (S1) to $\delta$-viniferin $(\mathbf{1 1 A}) .{ }^{1}$

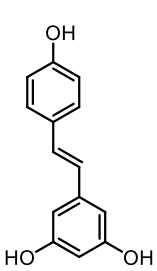

resveratrol (S1)

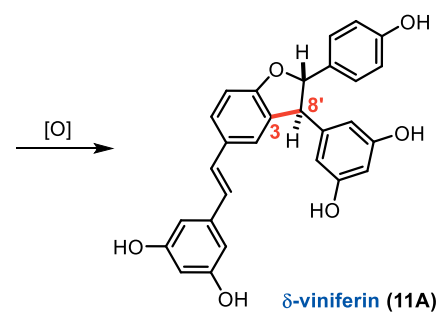

Yield

$16 \%$

$18 \%$

$$
\mathrm{DPPH}, \mathrm{MeOH}
$$

Various Peroxidases, Acetone/EtOH

Laccase, $n-\mathrm{BuOH}$

$13-21 \%$

$31 \%$

Galvinoxyl radical, ethanol, $\mathrm{rt}$

Graphitic carbon nitride ( $h v, 410$ $\mathrm{nm})$, lutidine, air, $\mathrm{MeCN}, \mathrm{rt}$

8

9

10

11
AgOAc, $\mathrm{MeOH}, 50{ }^{\circ} \mathrm{C}$

$\mathrm{MnO}_{2}, \mathrm{CH}_{2} \mathrm{Cl}_{2}$, rt

$\mathrm{HRP} / \mathrm{H}_{2} \mathrm{O}_{2}, \mathrm{H}_{2} \mathrm{O} /$ Acetone, $\mathrm{pH} 8$

$\mathrm{FeCl}_{3}$, Acetone, $\mathrm{rt}$

\section{$41 \%$}

$85 \%$

$86 \%$

$91 \%$

$93 \%$

$97 \%$
Reference

Zhou, B. et. al. Bioorg. Med. Chem. 2009, 17, 2360.

Ho, C.-T. et. al. J. Agric. Food Chem. 1999, 47, 3974.

Niwa, M. et. al. Tetrahedron 2005, 61, 10285.

Forti, L. et. al. Tetrahedron 2004, 60, 595.

Zhou, B. et. al. J. Org. Chem. 2009, 74, 5025.

Wang, Y.et. al. Chem.-Eur. J. 2014, 20, 678.

Iinuma, M. et. al. J. Org. Chem. 2004, 69, 2598.

Niwa, M. et. al. Tetrahedron 2005, 61, 10285.

Pan, Y. et. al. Green Chem. 2012, 14, 3281.

Niwa, M. et. al. Tetrahedron 2005, 61, 10285.

*Entries are organized by reaction yield. 


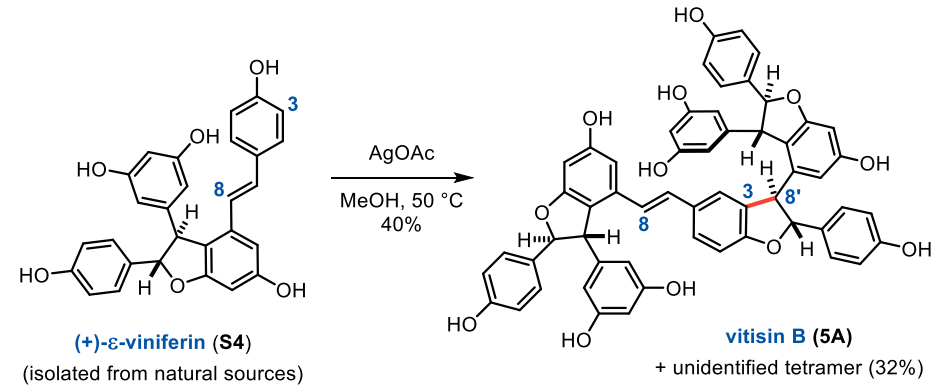

Figure S2. Sako and co-worker's semisynthesis of vitisin B (5A) from isolated (+)-ع-viniferin. ${ }^{2}$

\section{Van't Hoff Analysis of the 8A-9A-10A equilibrium}
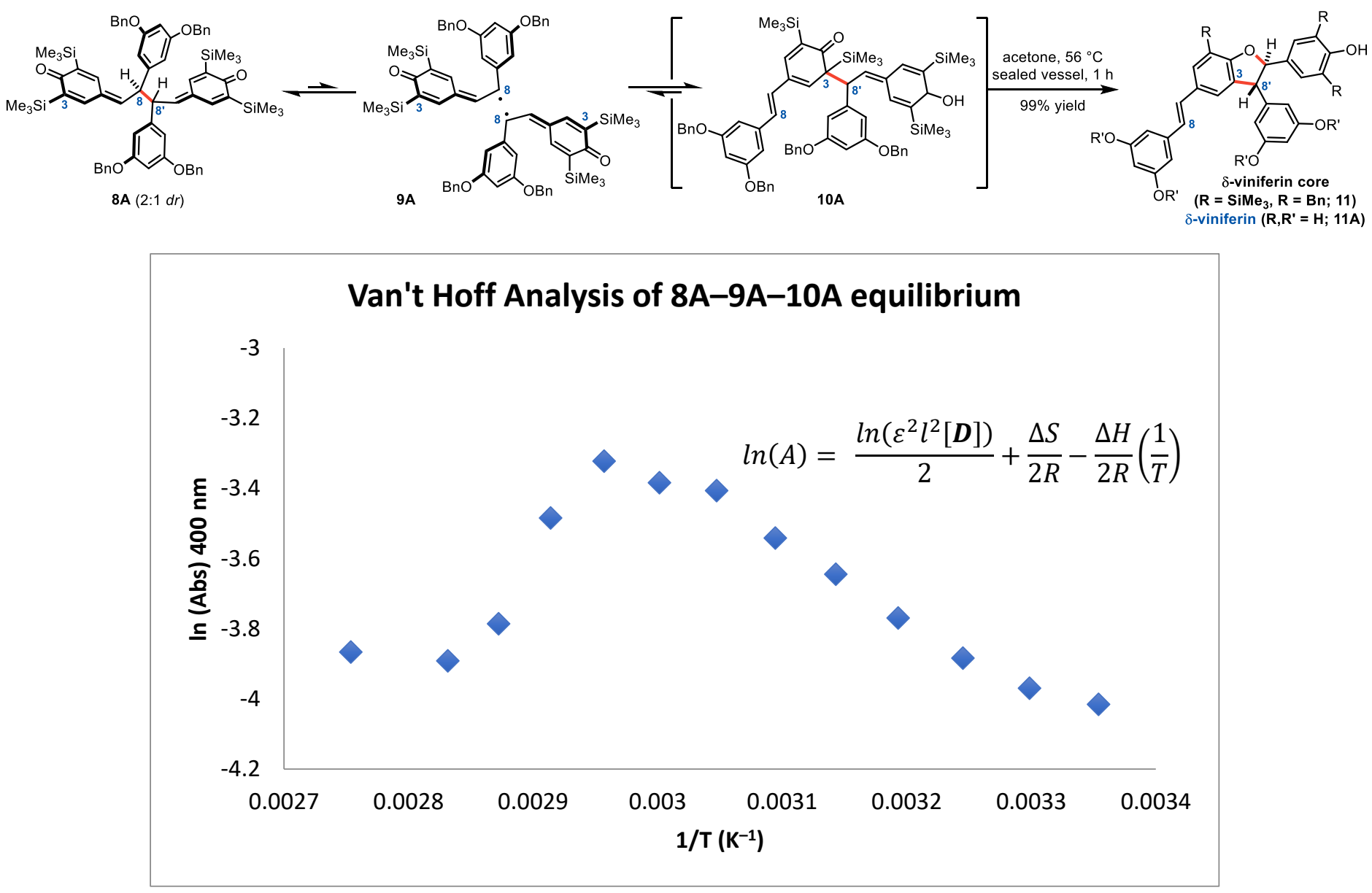

Figure S3. Van't Hoff analysis of the 8A-9A-10A equilibrium from differential temperature UV-Vis spectrometry.

UV-Vis/EPR: The temperature dependent UV-Vis measurements were performed as follows: To a $3.5 \mathrm{~mL}$ quartz cuvette was added $2.475 \mathrm{~mL}$ of 1,2-dichlorobenzene (dried over sieves) and $25 \mu \mathrm{L}$ of a $5 \mathrm{mM}$ stock of substrate in 1,2dichlorobenzene. The cuvette was capped with a rubber septum and purged with nitrogen for 10 minutes. The cuvette was connected to a nitrogen balloon to maintain a pressure of nitrogen throughout the experiment. A cuvette containing only solvent was used as a baseline throughout the experiment to record baseline-corrected data at each temperature. The cuvette was loaded into the multi-cell holder and allowed to equilibrate to each recorded temperature for at least 5 minutes before the absorbance reading. To determine the radical's extinction coefficient, a UV-Vis spectrum of a $50 \mu \mathrm{M}$ sample in benzene was measured and the solution then transferred into an EPR tube where the radical concentration was determined by EPR at the same temperature. Substitution of Beer's law into the Van't Hoff equation affords an inverse relationship between the natural $\log$ of UV-Vis absorbance and temperature. The inflection point in Figure S3 suggests that the 8A-9A-10A equilibrium is no longer reversible at elevated temperature. 
Experimental Procedures and Characterization Data:

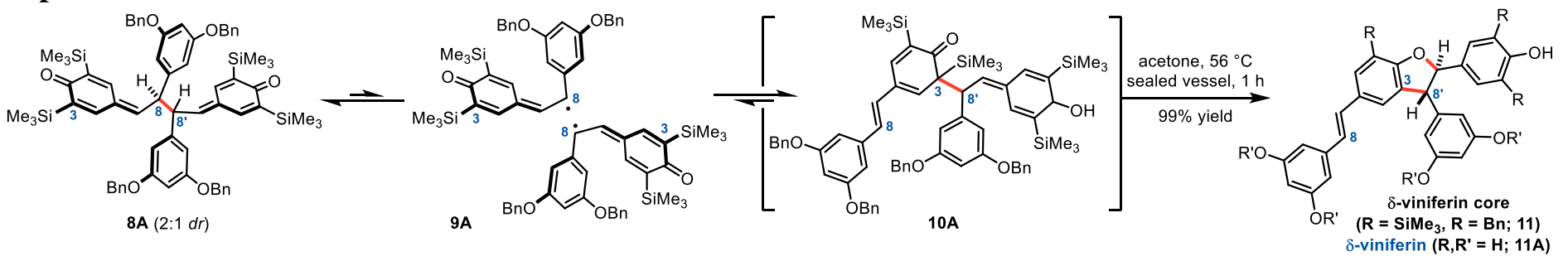

11 - 4-((2R)-3-(3,5-bis(benzyloxy)phenyl)-5-((E)-3,5-bis(benzyloxy)styryl)-7-(trimethylsilyl)-2,3dihydrobenzofuran-2-yl)-2,6-bis(trimethylsilyl)phenol

Quinone methide dimer 8A $\left(80 \mathrm{mg}, 0.0725 \mathrm{mmol}\right.$; obtained from our prior report $\left.{ }^{3}\right)$ was dissolved in acetone $(1.5 \mathrm{~mL}, \mathrm{HPLC}$ grade) in a 2-dram vial charged with a stir bar. The yellow solution was vigorously sparged with a balloon of argon for $>5$ min to exclude oxygen, and the reaction vial was capped and sealed with parafilm. The vial was heated at $56{ }^{\circ} \mathrm{C}$ for 1 hour, during which the solution transitioned from yellow to colorless. After 1 hour had passed, the reaction was cooled to room temperature, the stir bar was removed, and the solvent was evaporated under reduced pressure to afford $\delta$-viniferin core 11 (74 mg, 99\% yield) as an off-white foam. Note: The key to excellent yield for this reaction is the exclusion of oxygen. If not rigorously degassed, disproportionation of $\mathbf{8 A}$ occurs as a minor reaction pathway to return half of the dimeric material to the stilbene precursor and resulting in decomposition of the other half. Compound $\mathbf{1 1}$ was characterized in a prior report from our group. ${ }^{4}$ This same protocol was followed for the crossover experiment between 8A and S5 (see Figure S4).

${ }^{1} \mathrm{H}$ NMR (500 MHz, Chloroform- $d$ ) $\delta 7.47-7.28$ (m, 20H), 7.39 (s, overlap, 1H, C6-H), 7.27 (s, 2H, C2'-H), 7.19 (br s, $1 \mathrm{H}, \mathrm{C} 2-\mathrm{H}), 7.03(\mathrm{~d}, J=16.3 \mathrm{~Hz}, 1 \mathrm{H}, \mathrm{C} 7-\mathrm{H}), 6.82(\mathrm{~d}, J=16.3 \mathrm{~Hz}, \mathrm{C} 8-\mathrm{H}), 6.74(\mathrm{~d}, J=2.2 \mathrm{~Hz}, 2 \mathrm{H}, \mathrm{C} 10-\mathrm{H}), 6.60(\mathrm{t}, J=2.2$ $\left.\mathrm{Hz}, 1 \mathrm{H}, \mathrm{C} 12^{\prime}-\mathrm{H}\right), 6.52(\mathrm{t}, J=2.2 \mathrm{~Hz}, 1 \mathrm{H}, \mathrm{C} 12-\mathrm{H}), 6.50\left(\mathrm{~d}, J=2.2 \mathrm{~Hz}, 2 \mathrm{H}, \mathrm{C} 10^{\prime}-\mathrm{H}\right), 5.51\left(\mathrm{~d}, J=9.2 \mathrm{~Hz}, 1 \mathrm{H}, \mathrm{C} 7^{\prime}-\mathrm{H}\right), 5.07$ $\left(\mathrm{s}, 4 \mathrm{H}, \mathrm{C} 11 \mathrm{a}-\mathrm{OCH}_{2} \mathrm{C}_{6} \mathrm{H}_{5}\right), 5.03\left(\mathrm{~s}, 1 \mathrm{H}, \mathrm{C}^{\prime}-\mathrm{OH}\right), 5.00\left(\mathrm{~s}, 4 \mathrm{H}, \mathrm{C} 11^{\prime}-\mathrm{H}\right), 4.35\left(\mathrm{~d}, J=9.2 \mathrm{~Hz}, 1 \mathrm{H}, \mathrm{C} 8^{\prime}-\mathrm{H}\right), 0.39(\mathrm{~s}, 9 \mathrm{H}, \mathrm{C} 5-$ $\left.\mathrm{Si}\left(\mathrm{CH}_{3}\right)_{3}\right), 0.31\left(\mathrm{~s}, 18 \mathrm{H}, \mathrm{C} 3 / 5^{\prime}-\mathrm{Si}\left(\mathrm{CH}_{3}\right)_{3}\right)$

${ }^{13} \mathrm{C}$ NMR (126 MHz, Chloroform- $d$ ) $\delta 165.24$ (C4), 165.19 (C4'), 160.5 (C11'), 160.4 (C11), 144.3 (C9'), 140.2 (C9), 137.2 $\left(-\mathrm{OCH}_{2} \mathrm{C}_{6} \mathrm{H}_{5}\right), 136.9\left(-\mathrm{OCH}_{2} \mathrm{C}_{6} \mathrm{H}_{5}\right), 133.8\left(\mathrm{C}^{\prime}\right), 133.6(\mathrm{C} 6), 132.8\left(\mathrm{Cl}^{\prime}\right), 130.4(\mathrm{C} 1), 129.7(\mathrm{C} 7), 129.6(\mathrm{C} 3), 128.8(-$ $\left.\mathrm{OCH}_{2} \mathrm{C}_{6} \mathrm{H}_{5}\right), 128.3\left(-\mathrm{OCH}_{2} \mathrm{C}_{6} \mathrm{H}_{5}\right), 128.2\left(-\mathrm{OCH}_{2} \mathrm{C}_{6} \mathrm{H}_{5}\right), 127.9\left(-\mathrm{OCH}_{2} \mathrm{C}_{6} \mathrm{H}_{5}\right), 127.8\left(-\mathrm{OCH}_{2} \mathrm{C}_{6} \mathrm{H}_{5}\right), 126.0(\mathrm{C} 8), 124.4\left(\mathrm{C} 3^{\prime}\right)$, 124.0 (C2), 120.3 (C5), $108.1\left(\mathrm{C} 10^{\prime}\right), 105.6(\mathrm{C} 10), 101.4(\mathrm{C} 12), 100.8\left(\mathrm{C} 12^{\prime}\right), 92.6\left(\mathrm{C}^{\prime}\right), 70.4\left(\mathrm{C}_{1} 1^{\prime}-\mathrm{OCH}_{2} \mathrm{C}_{6} \mathrm{H}_{5}\right), 70.3$ $\left(\mathrm{C} 11-\mathrm{OCH}_{2} \mathrm{C}_{6} \mathrm{H}_{5}\right), 58.3\left(\mathrm{C} 8^{\prime}\right),-0.3\left(\mathrm{C} 3 / 5^{\prime}-\mathrm{Si}\left(\mathrm{CH}_{3}\right)_{3}\right),-0.9\left(\mathrm{C} 5-\mathrm{Si}\left(\mathrm{CH}_{3}\right)_{3}\right)$

IR (Neat): 3606, 2951, 1586, 1497, 1454, 1398, 1294, 1244, 1149, 1057, 1029, 960, 905, 834, 733, $694 \mathrm{~cm}^{-1}$.

HRMS (ESI) $m / z$ calculated for $\mathrm{C}_{65} \mathrm{H}_{71} \mathrm{O}_{6} \mathrm{Si}_{3}{ }^{+}\left([\mathrm{M}+\mathrm{H}]^{+}\right)$1031.4533, found 1031.4528 . 

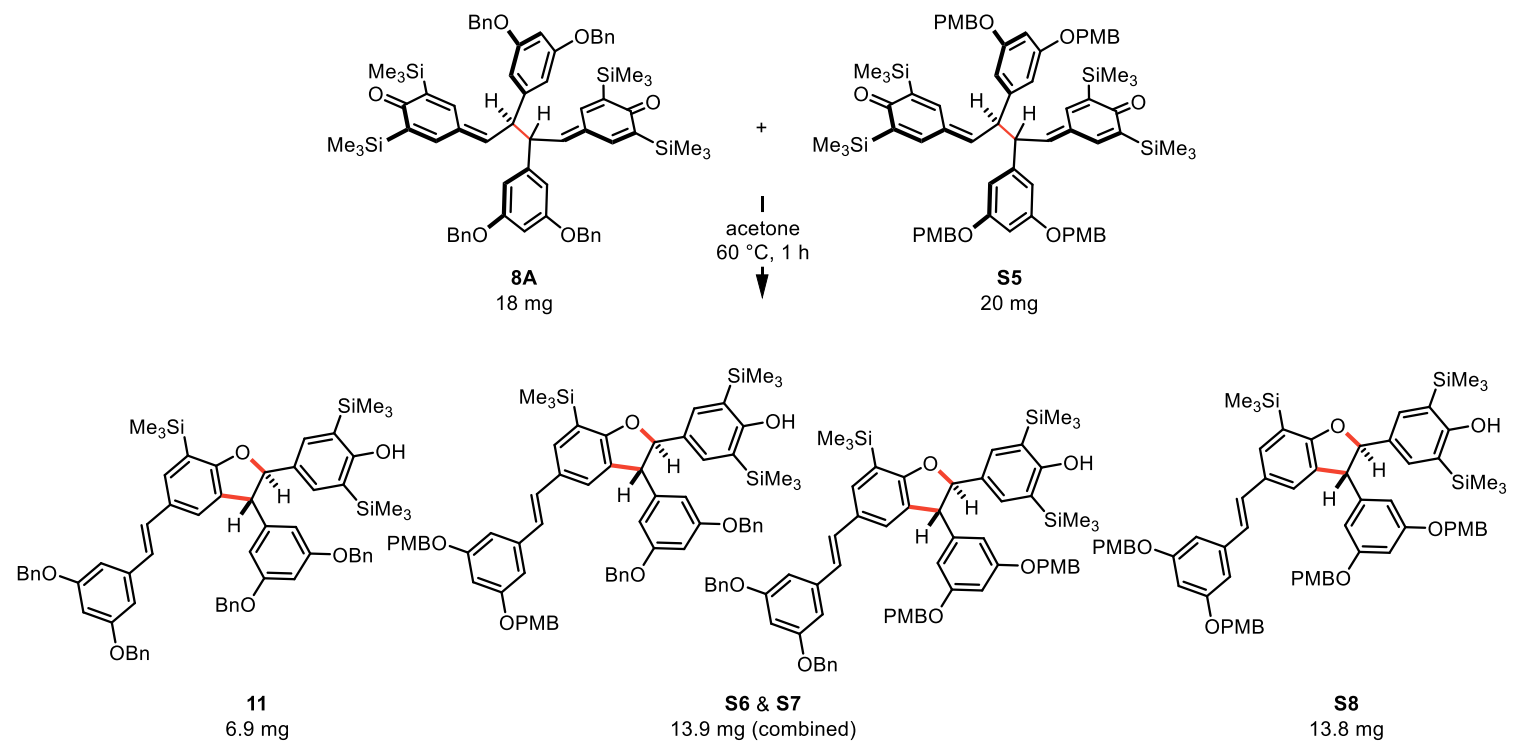

Figure S4. Crossover experiment between $\mathbf{8 A}$ and $\mathbf{S 5}$ demonstrates that the persistent radicals escape the solvent cage during the isomerization reaction.

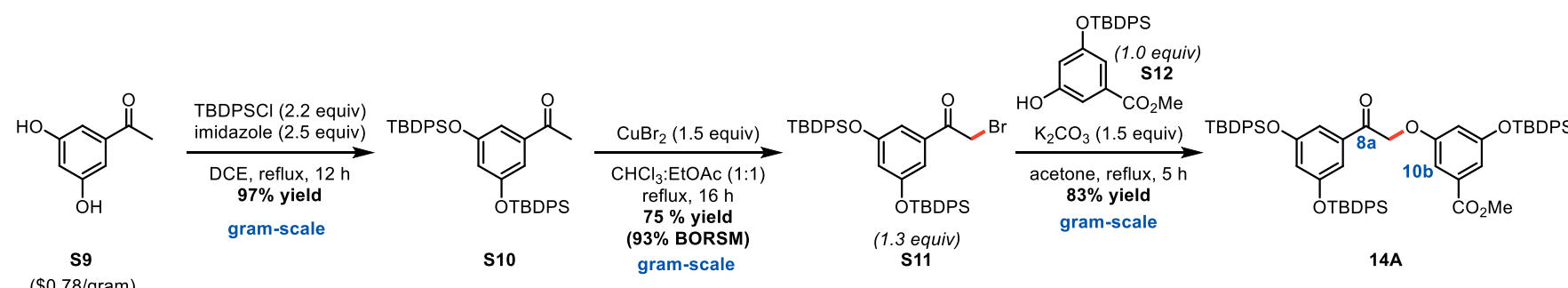

(\$0.78/gram)

gram-scale

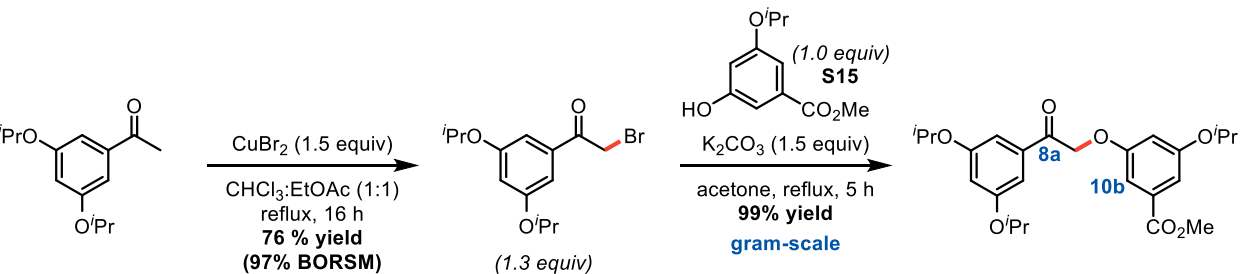

S13

gram-scale

S14

14B

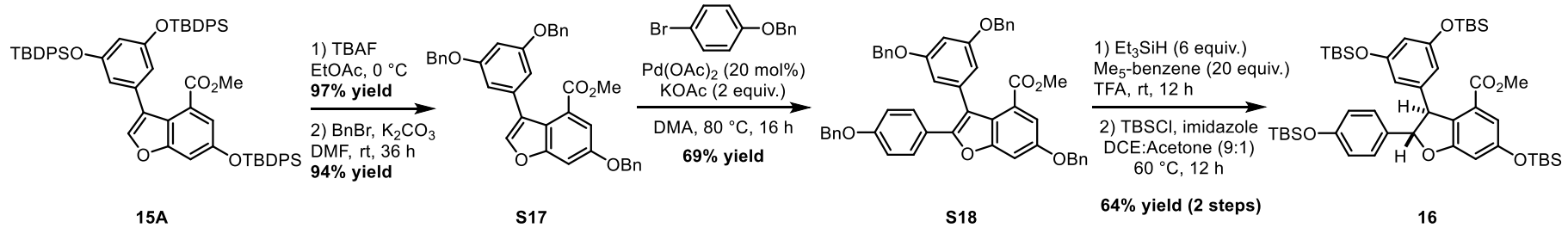

Figure S5. Preparation of acetophenone 14A and 14B, and alternate preparation of 16. 


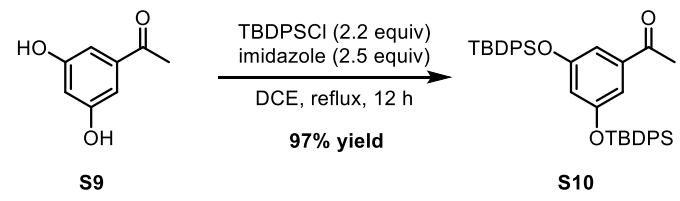

\section{S10 - 1-(3,5-bis((tert-butyldiphenylsilyl)oxy)phenyl)ethan-1-one}

The starting acetophenone $\mathbf{S 9}(5.0 \mathrm{~g}, 32.9 \mathrm{mmol})$ and imidazole $(5.0 \mathrm{~g}, 73.9 \mathrm{mmol}, 2.25$ equiv) were added to a flame-dried round bottom flask fitted with a reflux condenser and charged with a stir bar under inert atmosphere. The solids were suspended in 1,2-dichloroethane $(115 \mathrm{~mL})$, and to the stirring suspension was added TBDPSCl (18.8 $\mathrm{mL}, 72.3 \mathrm{mmol}, 2.2$ equiv) via syringe. The reaction mixture was heated at reflux for 12 hours; after cooling to room temperature, the heterogeneous reaction mixture was filtered over Celite, concentrated, and purified by column chromatography $(0-20 \%$ EtOAc in Hexanes) to afford the desired product $\mathbf{S 1 0}$ as a colorless solid (20 g, 97\% yield).

${ }^{1} \mathrm{H}$ NMR $(700 \mathrm{MHz}$, Chloroform- $d$ ) $\delta 7.57(\mathrm{~d}, J=7.6 \mathrm{~Hz}, 8 \mathrm{H}), 7.40(\mathrm{t}, J=7.4 \mathrm{~Hz}, 4 \mathrm{H}), 7.30(\mathrm{t}, J=7.0 \mathrm{~Hz}, 8 \mathrm{H}), 6.89(\mathrm{~d}, J$ $=2.5 \mathrm{~Hz}, 2 \mathrm{H}), 6.39(\mathrm{~d}, J=2.3 \mathrm{~Hz}, 1 \mathrm{H}), 2.19(\mathrm{~s}, 3 \mathrm{H}), 1.04(\mathrm{~s}, 18 \mathrm{H})$.

${ }^{13} \mathrm{C}$ NMR (176 MHz, Chloroform- $d$ ) $\delta$ 197.7, 156.5, 138.6, 135.6, 132.5, 130.0, 127.9, 116.4, 113.2, 26.6 (2C), 19.6.

IR (Neat): 2977, 1684, 1589, 1556, 1364, 1298, 1187, 1151, $1110 \mathrm{~cm}^{-1}$.

HRMS (ESI) $m / z$ calculated for $\mathrm{C}_{40} \mathrm{H}_{45} \mathrm{O}_{3} \mathrm{Si}_{2}{ }^{+}\left([\mathrm{M}+\mathrm{H}]^{+}\right)$629.2902, found 629.2901.

${ }^{1} \mathrm{H}$ NMR, $700 \mathrm{MHz}$, Chloroform-d, Compound S10
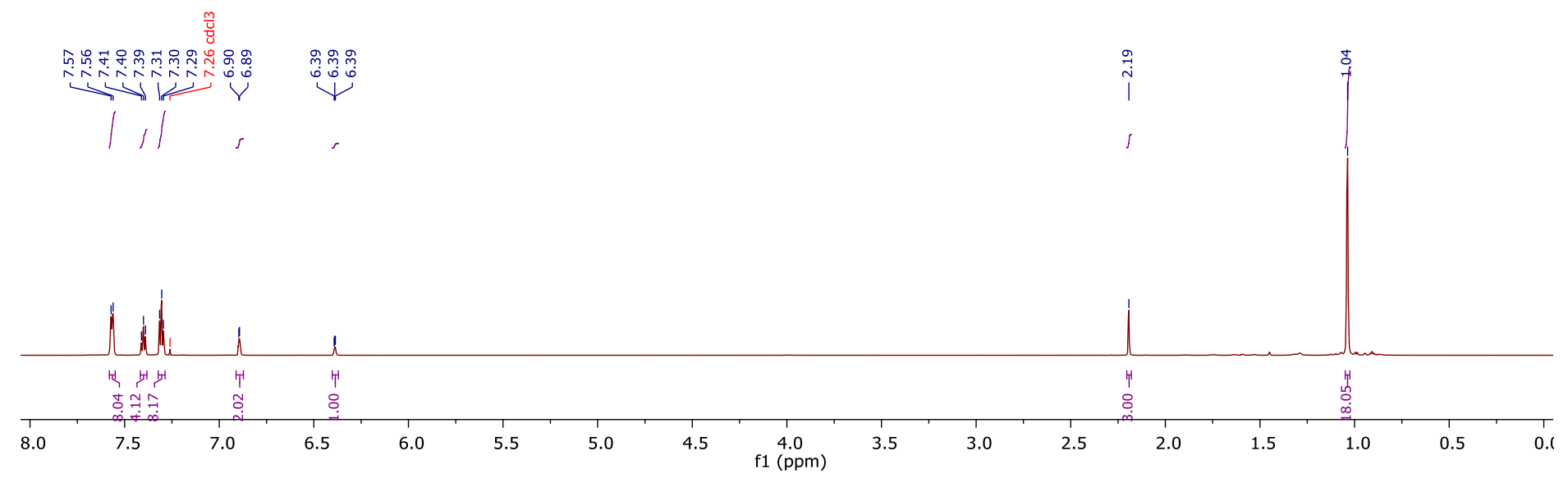

${ }^{13} \mathrm{C}$ NMR, $176 \mathrm{MHz}$, Chloroform- $d$, Compound S10

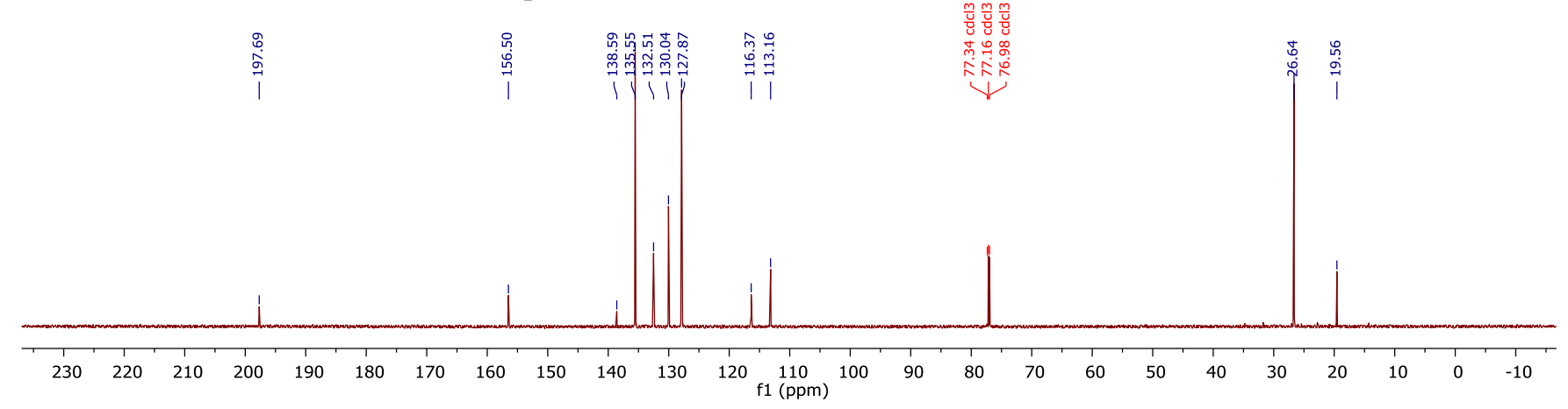




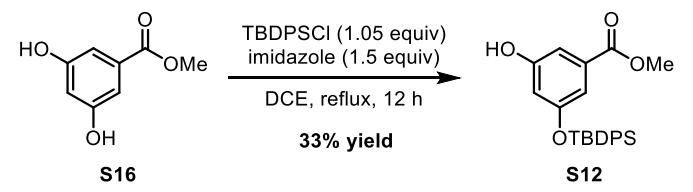

\section{S12 - methyl 3-((tert-butyldiphenylsilyl)oxy)-5-hydroxybenzoate}

The starting acetophenone $\mathbf{S 1 6}$ (2.0 g, $11.9 \mathrm{mmol})$ and imidazole (1.2 g, $1.8 \mathrm{mmol}, 1.5$ equiv) were added to a flame-dried round bottom flask fitted with a reflux condenser and charged with a stir bar under inert atmosphere. The solids were suspended in 1,2-dichloroethane $(100 \mathrm{~mL})$, and to the stirring suspension was added TBDPSCl (3.25 mL, $1.25 \mathrm{mmol}, 1.05$ equiv) via syringe. The reaction mixture was heated at reflux for 12 hours; after cooling to room temperature, the heterogeneous reaction mixture was filtered over Celite, concentrated, and purified by column chromatography $(0-40 \%$ EtOAc in Hexanes) to afford the desired product $\mathbf{S 1 2}$ as a white solid (1.63 g, 33\% yield).

${ }^{1} \mathrm{H}$ NMR $(500 \mathrm{MHz}$, Chloroform- $d$ ) $\delta 7.70(\mathrm{~d}, J=6.6 \mathrm{~Hz}, 4 \mathrm{H}), 7.43(\mathrm{t}, J=7.3 \mathrm{~Hz}, 2 \mathrm{H}), 7.37(\mathrm{t}, J=7.2 \mathrm{~Hz}, 4 \mathrm{H}), 7.10(\mathrm{dd}$, $J=2.2,1.4 \mathrm{~Hz}, 1 \mathrm{H}), 7.05(\mathrm{dd}, J=2.3,1.4 \mathrm{~Hz}, 1 \mathrm{H}), 6.36(\mathrm{t}, J=2.3 \mathrm{~Hz}, 1 \mathrm{H}), 5.06(\mathrm{~s}, 1 \mathrm{H}), 3.81(\mathrm{~s}, 3 \mathrm{H}), 1.10(\mathrm{~s}, 9 \mathrm{H})$.

${ }^{13} \mathrm{C}$ NMR (126 MHz, Chloroform- $d$ ) $\delta 166.9,156.9,156.3,135.6,132.5,132.0,130.2,128.0,114.0,111.8,109.6,52.3$, 26.6, 19.6 .

IR (Neat): 3423 (br), 2974, 1724, 1586, 1364, 1298, 1182, 1156, $1124 \mathrm{~cm}^{-1}$.

HRMS (ESI) $m / z$ calculated for $\mathrm{C}_{24} \mathrm{H}_{27} \mathrm{O}_{4} \mathrm{Si}^{+}\left([\mathrm{M}+\mathrm{H}]^{+}\right)$407.1673, found 407.1675.

${ }^{1} \mathrm{H}$ NMR, $500 \mathrm{MHz}$, Chloroform- $d$, Compound S12
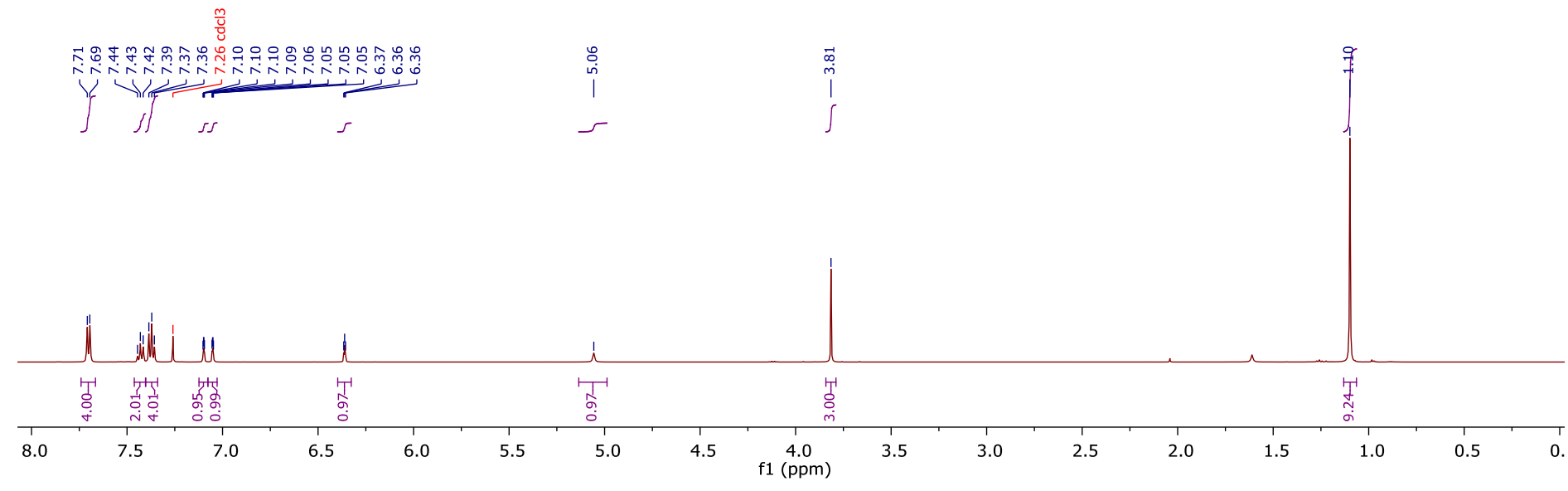

${ }^{13} \mathrm{C}$ NMR, $126 \mathrm{MHz}$, Chloroform- $d$, Compound $\mathbf{S 1 2}$

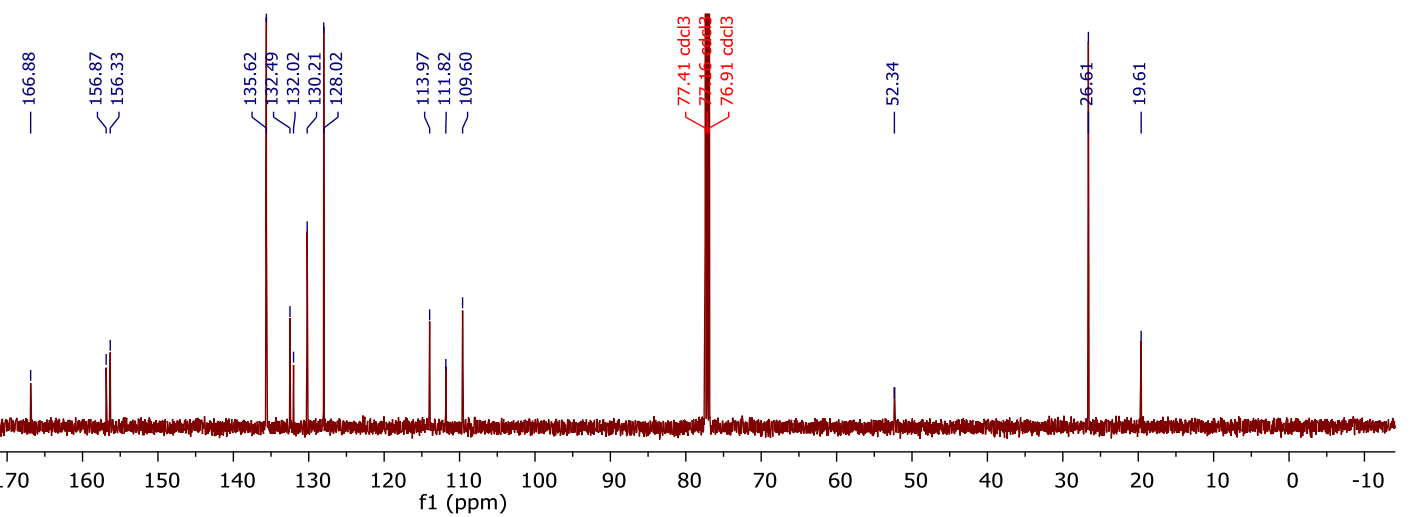




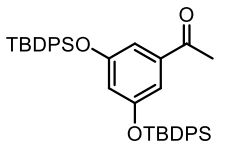

S10

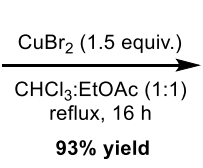

$93 \%$ yield

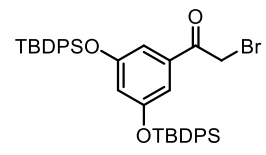

s11

\section{S11 - 1-(3,5-bis((tert-butyldiphenylsilyl)oxy)phenyl)-2-bromoethan-1-one}

The starting acetophenone $\mathbf{S 1 0}(7.50 \mathrm{~g}, 11.9 \mathrm{mmol})$ was added to a flame-dried round bottom flask fitted with a reflux condenser and charged with a stir bar under inert atmosphere. The starting material was dissolved in a 1:1 solution of $\mathrm{CHCl}_{3}$ and EtOAc $(100 \mathrm{~mL})$, and $\mathrm{CuBr}_{2}(4.00 \mathrm{~g}, 17.9 \mathrm{mmol}, 1.5$ equiv $)$ was added to the reaction solution. The reaction was heated at reflux overnight $(\sim 16 \mathrm{~h})$, at which point it was cooled to rt and filtered through a plug of Celite in the fume hood, eluting with DCM $(\sim 100 \mathrm{~mL})$. The filtrate was transferred to a separatory funnel containing sat'd aq. $\mathrm{NaHCO}_{3}(100 \mathrm{~mL})$. The layers were separated, and the organic layer was washed with aqueous solution of $10 \%$ sodium thiosulphate $(100 \mathrm{~mL})$. The fully quenched organic layer was subsequently washed with brine, dried over $\mathrm{Na}_{2} \mathrm{SO}_{4}$, and concentrated under reduced pressure. The crude product was purified by flash column chromatography (5\% to 40\% DCM/Hexanes) to afford the desired brominated product $\mathbf{S 1 1}$ as a white solid (6.3 g, 75\% yield) in addition to unreacted $\mathbf{S 1 0}$ as a clear, colorless oil (1.50 g, $2.38 \mathrm{mmol}$ ). The yield based on recovered $\mathbf{S 1 0}$ is $93 \%$.

${ }^{1} \mathrm{H}$ NMR $(500 \mathrm{MHz}$, Chloroform- $d$ ) $\delta 7.55(\mathrm{~d}, J=7.3 \mathrm{~Hz}, 8 \mathrm{H}), 7.40(\mathrm{t}, J=7.1 \mathrm{~Hz}, 4 \mathrm{H}), 7.30(\mathrm{t}, J=7.4 \mathrm{~Hz}, 8 \mathrm{H}), 6.84(\mathrm{~d}, J$ $=2.2 \mathrm{~Hz}, 2 \mathrm{H}), 6.42(\mathrm{~d}, J=2.3 \mathrm{~Hz}, 1 \mathrm{H}), 3.96(\mathrm{~s}, 2 \mathrm{H}), 1.02(\mathrm{~s}, 18 \mathrm{H})$.

${ }^{13} \mathrm{C}$ NMR (126 MHz, Chloroform- $d$ ) $\delta$ 190.6, 156.8, 135.6, 135.3, 132.4, 130.2, 128.0, 117.4, 113.6, 31.5, 26.6, 19.6.

IR (Neat): 2977, 1684, 1589, 1556, 1364, 1298, 1187, 1151, 1110, $742 \mathrm{~cm}^{-1}$.

HRMS (ESI) $m / z$ calculated for $\mathrm{C}_{40} \mathrm{H}_{44} \mathrm{BrO}_{3} \mathrm{Si}_{2}{ }^{+}\left([\mathrm{M}+\mathrm{H}]^{+}\right)$707.2007, found 707.2001

${ }^{1} \mathrm{H}$ NMR, $500 \mathrm{MHz}$, Chloroform- $d$, Compound S11
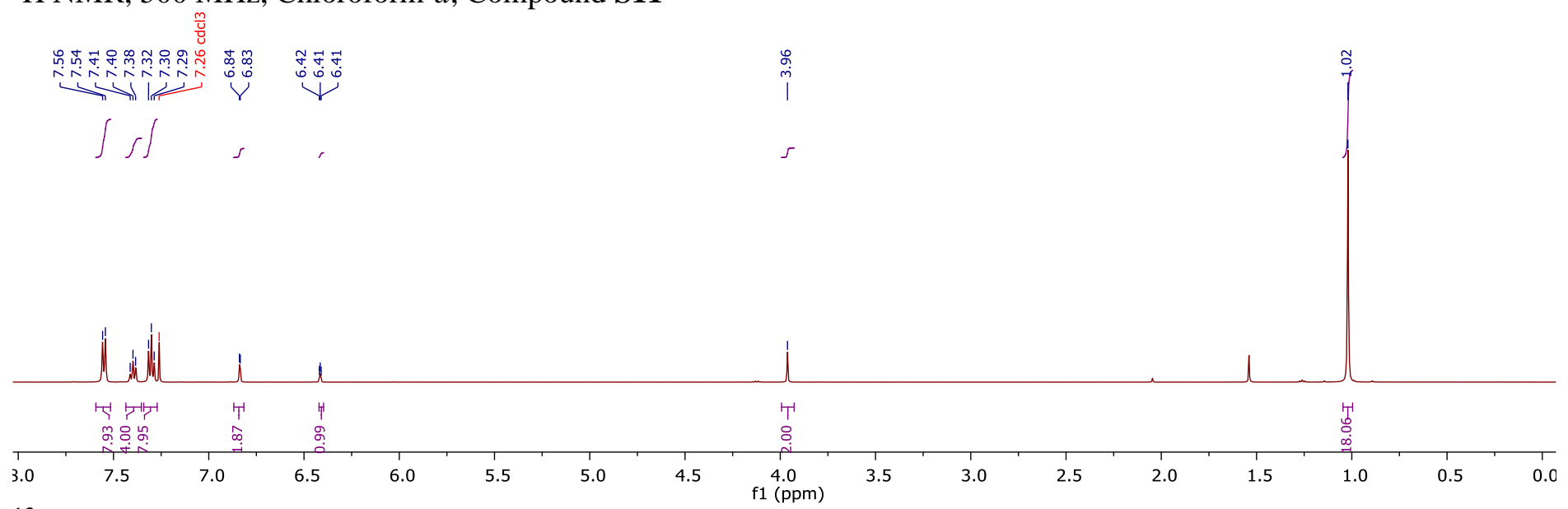

${ }^{13} \mathrm{C}$ NMR, $126 \mathrm{MHz}$, Chloroform- $d$, Compound S11

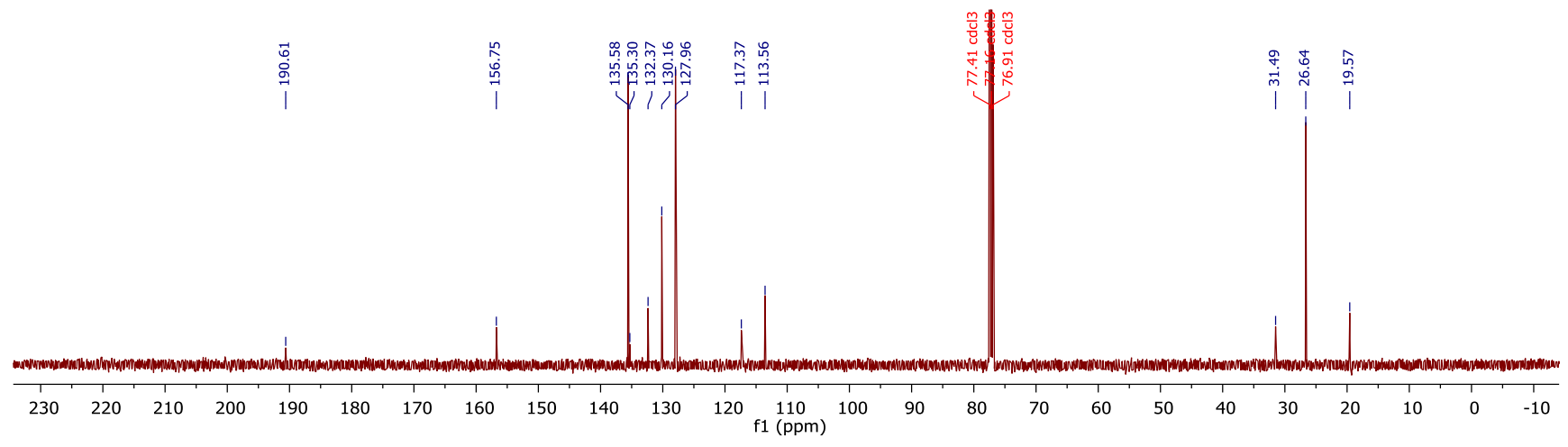




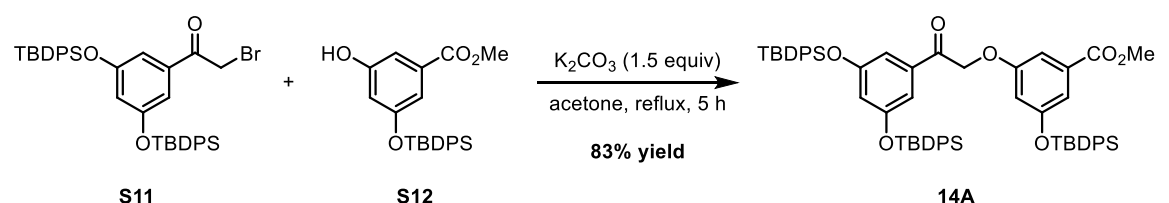

\section{A - methyl 3-(2-(3,5-bis((tert-butyldiphenylsilyl)oxy)phenyl)-2-oxoethoxy)-5-((tert- butyldiphenylsilyl)oxy)benzoate}

A solution of the starting phenol $\mathbf{S 1 2}(0.65 \mathrm{~g}, 1.60 \mathrm{mmol}, 1.0$ equiv) and bromide $\mathbf{S 1 1}$ (1.30 g, $1.84 \mathrm{mmol}, 1.15$ equiv) in acetone $(30 \mathrm{~mL})$ was prepared in a flame-dried, $100 \mathrm{~mL}$ rb flask fitted with reflux condenser under inert atmosphere. To the stirring solution was added $\mathrm{K}_{2} \mathrm{CO}_{3}(0.331 \mathrm{~g}, 2.40 \mathrm{mmol}, 1.5$ equiv) in a single portion, and the reaction was heated to reflux for $5 \mathrm{~h}$. Upon cooling to $\mathrm{rt}$, the crude reaction was concentrated under reduced pressure. The residue was dissolved in EtOAc and added to a sep. funnel containing DI $\mathrm{H}_{2} \mathrm{O}$. The layers were separated, and the organic layer was washed with brine, dried over $\mathrm{MgSO}_{4}$, and concentrated. The crude product was purified by flash column chromatography $(1 \%, 2 \%, 3 \%, 5 \%$, $8 \%, 12 \%, 18 \%$ EtOAc in Hexanes, $1.5 \mathrm{CV}$ per step, $\mathrm{CV}=200 \mathrm{~mL})$ to afford the product $14 \mathrm{~A}$ as a white foam $(1.37 \mathrm{~g}, 1.33$ mmol, $83 \%$ yield).

${ }^{1} \mathrm{H}$ NMR $(500 \mathrm{MHz}$, Chloroform- $d$ ) $\delta 7.66(\mathrm{~d}, J=6.4 \mathrm{~Hz}, 4 \mathrm{H}), 7.53(\mathrm{~d}, J=6.5 \mathrm{~Hz}, 8 \mathrm{H}), 7.37(\mathrm{t}, J=7.3 \mathrm{~Hz}, 6 \mathrm{H}), 7.32(\mathrm{~d}, J$ $=7.7 \mathrm{~Hz}, 4 \mathrm{H}), 7.28(\mathrm{t}, J=7.5 \mathrm{~Hz}, 8 \mathrm{H}), 7.12(\mathrm{t}, J=1.7 \mathrm{~Hz}, 1 \mathrm{H}), 7.02-6.96(\mathrm{~m}, 1 \mathrm{H}), 6.73(\mathrm{~d}, J=2.2 \mathrm{~Hz}, 2 \mathrm{H}), 6.40(\mathrm{t}, J=$ $2.2 \mathrm{~Hz}, 1 \mathrm{H}), 6.36(\mathrm{t}, J=2.4 \mathrm{~Hz}, 1 \mathrm{H}), 4.53(\mathrm{~s}, 2 \mathrm{H}), 3.82(\mathrm{~s}, 3 \mathrm{H}), 1.10(\mathrm{~s}, 9 \mathrm{H}), 1.02(\mathrm{~s}, 18 \mathrm{H})$.

${ }^{13} \mathrm{C}$ NMR $(126 \mathrm{MHz}$, Chloroform- $d$ ) $\delta$ 192.7, 166.7, 158.7, 156.7, 156.7, 135.6, 135.6, 132.5, 132.3, 131.9, 130.20, 130.18, 128.0, 127.9, 117.2, 114.8, 113.8, 112.6, 111.7, 108.7, 70.3, 52.2, 26.70, 26.66, 19.64, 19.57.

IR (Neat): 2977, 1724, 1684, 1589, 1556, 1364, 1298, 1187, 1151, $1110 \mathrm{~cm}^{-1}$.

HRMS (ESI) $m / z$ calculated for $\mathrm{C}_{64} \mathrm{H}_{69} \mathrm{O}_{7} \mathrm{Si}_{3}{ }^{+}\left([\mathrm{M}+\mathrm{H}]^{+}\right)$1033.4346, found 1033.4336 .

${ }^{1} \mathrm{H}$ NMR, $500 \mathrm{MHz}$, Chloroform- $d$, Compound 14A

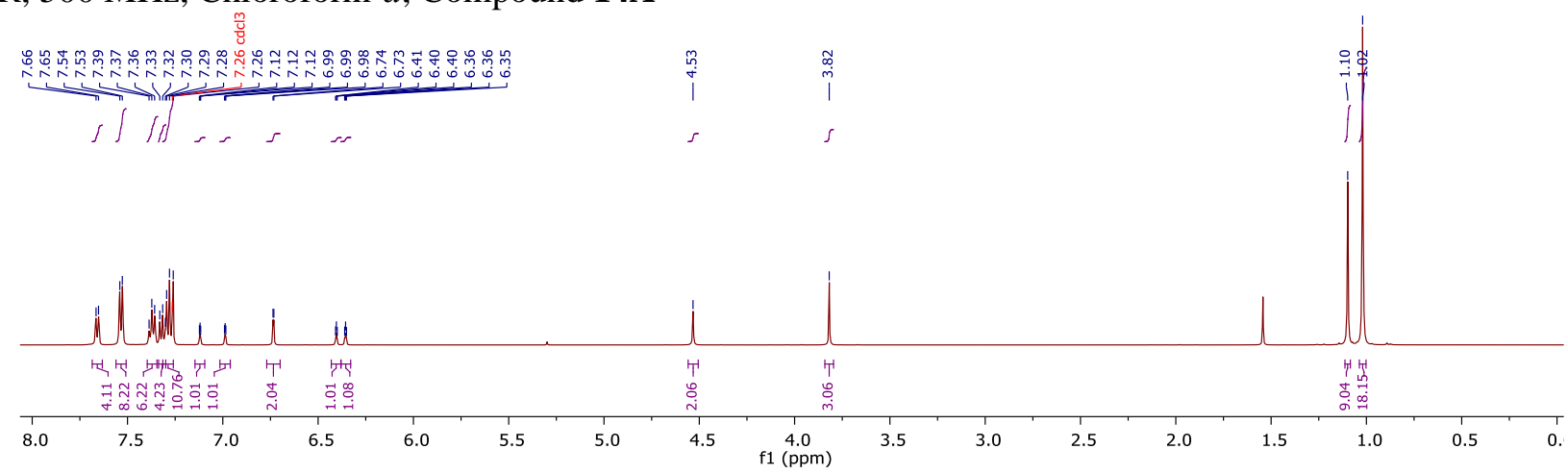

${ }^{13} \mathrm{C}$ NMR, $126 \mathrm{MHz}$, Chloroform- $d$, Compound 14A

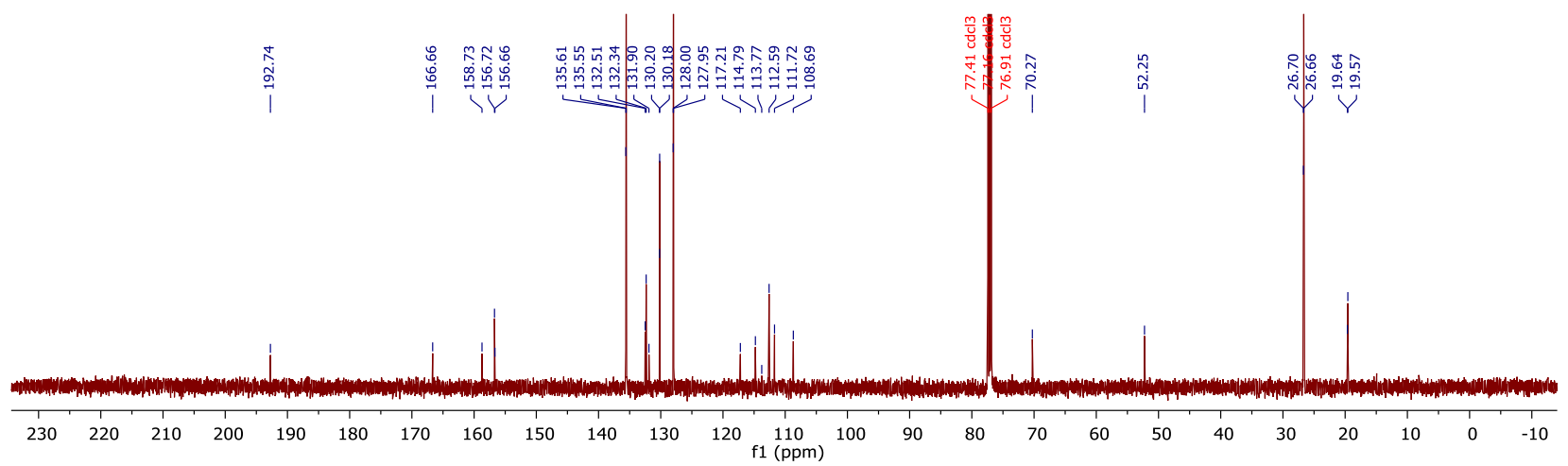




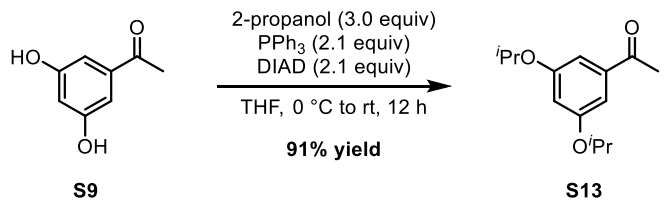

\section{S13 - 1-(3,5-diisopropoxyphenyl)ethan-1-one}

The starting acetophenone $\mathbf{S 9}$ (3.00 g, $19.7 \mathrm{mmol})$, 2-propanol (4.52 mL, $59.2 \mathrm{mmol}, 3.0$ equiv) and $\mathrm{PPh}_{3}(10.9 \mathrm{~g}, 41.4$ mmol, 2.10 equiv) were added to a flame-dried $250-\mathrm{mL}$ round bottom flask charged with a stir bar under inert atmosphere. The starting materials were dissolved in THF $(60 \mathrm{~mL})$, and to the stirring solution was cooled to $0{ }^{\circ} \mathrm{C}$ in an ice bath. To the cold solution was added DIAD $(8.15 \mathrm{~mL}, 41.4 \mathrm{mmol}, 2.10$ equiv) via syringe pump over 20 minutes. The reaction mixture was allowed to warm to room temperature over 12 hours, at which point the reaction was concentrated onto Celite and purified by column chromatography (0-15\% EtOAc in Hexanes) to afford the desired product $\mathbf{S 1 3}$ as a colorless oil (4.26 g, $91 \%$ yield).

${ }^{1} \mathrm{H}$ NMR $(500 \mathrm{MHz}$, Chloroform- $d$ ) $\delta 7.05(\mathrm{~d}, J=2.3 \mathrm{~Hz}, 2 \mathrm{H}), 6.62(\mathrm{t}, J=2.3 \mathrm{~Hz}, 1 \mathrm{H}), 4.58$ (hept, $J=6.1 \mathrm{~Hz}, 2 \mathrm{H}), 2.55(\mathrm{~s}$, $3 \mathrm{H}), 1.34(\mathrm{~d}, J=6.0 \mathrm{~Hz}, 12 \mathrm{H})$.

${ }^{13} \mathrm{C}$ NMR (126 MHz, Chloroform- $d$ ) $\delta$ 198.1, 159.2, 139.2, 108.6, 107.9, 70.3, 26.9, 22.1.

IR (Neat): 2977, 1684, 1589, 1362, 1295, 1184, 1156, $1114 \mathrm{~cm}^{-1}$.

HRMS (ESI) $m / z$ calculated for $\mathrm{C}_{14} \mathrm{H}_{21} \mathrm{O}_{3}{ }^{+}\left([\mathrm{M}+\mathrm{H}]^{+}\right)$237.1485, found 237.1496.

${ }^{1} \mathrm{H}$ NMR, $500 \mathrm{MHz}$, Chloroform-d, Compound S13

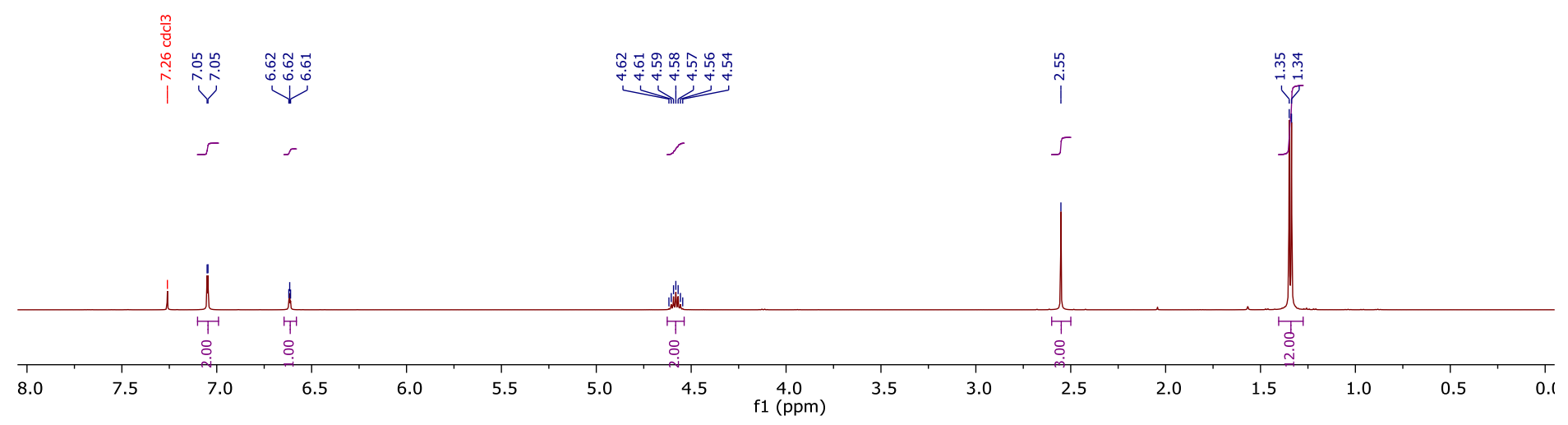

${ }^{13} \mathrm{C}$ NMR, $126 \mathrm{MHz}$, Chloroform- $d$, Compound S13

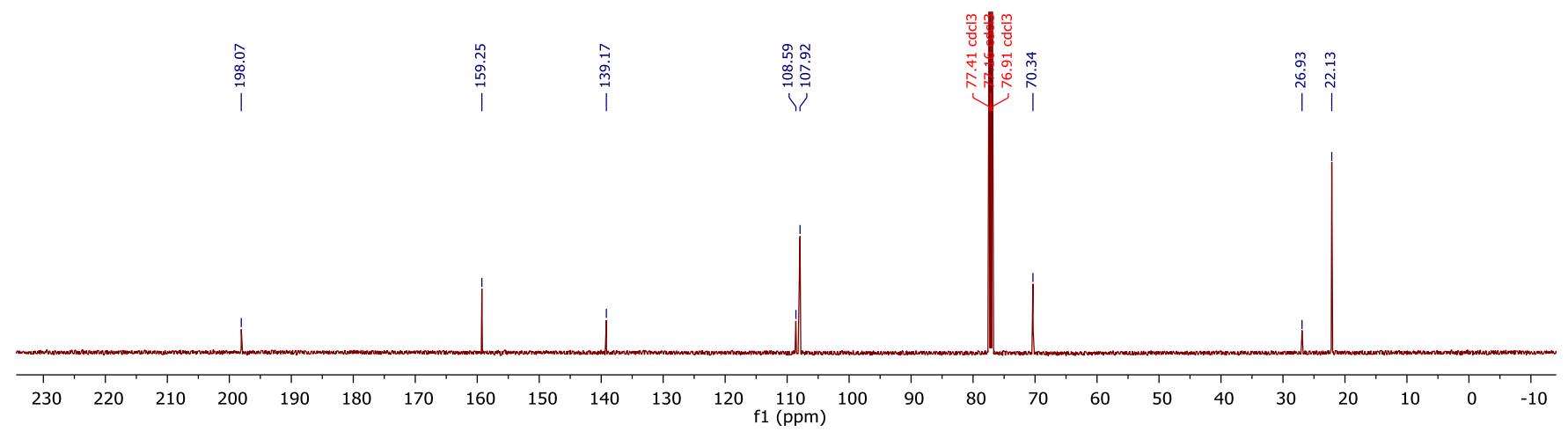




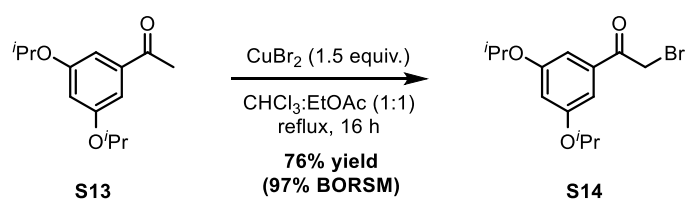

\section{S14 - 2-bromo-1-(3,5-diisopropoxyphenyl)ethan-1-one}

The starting acetophenone $\mathbf{S 1 3}(5.70 \mathrm{~g}, 24.1 \mathrm{mmol})$ was added to a flame-dried 500-mL round bottom flask fitted with a reflux condenser and charged with a stir bar under inert atmosphere. The starting material was dissolved in a 1:1 solution of $\mathrm{CHCl}_{3}$ and EtOAc (185 mL), and $\mathrm{CuBr}_{2}(8.08 \mathrm{~g}, 36.2 \mathrm{mmol}, 1.5$ equiv) was added to the reaction solution. The reaction was heated at reflux overnight $(\sim 16 \mathrm{~h})$, at which point it was cooled to $\mathrm{rt}$ and filtered through a plug of Celite in the fume hood, eluting with $\mathrm{CH}_{2} \mathrm{Cl}_{2}(\sim 100 \mathrm{~mL})$. The filtrate was transferred to a separatory funnel containing sat'd aq. $\mathrm{NaHCO}_{3}(100$ $\mathrm{mL}$ ). The layers were separated, and the organic layer was washed with aqueous solution of $10 \%$ sodium thiosulphate (100 $\mathrm{mL}$ ). The fully quenched organic layer was subsequently washed with brine, dried over $\mathrm{Na}_{2} \mathrm{SO}_{4}$, and concentrated under reduced pressure. The crude product was purified by flash column chromatography (1.5\% to $9 \%$ EtOAc/Hexanes over 6 steps) to afford the desired brominated product $\mathbf{S 1 4}$ as a colorless oil (5.75 g, 76\% yield) in addition to unreacted $\mathbf{S 1 3}$ as a clear, colorless oil (1.25 g, 22\% RSM). The yield based on recovered S13 is 97\%. At higher conversions, $\mathbf{S 1 4}$ reacts further to give the undesired dibrominated material, thus holding the reaction at $\sim 80 \%$ conversion was found to be optimal.

${ }^{1} \mathrm{H}$ NMR (401 MHz, Chloroform-d) $\delta 7.05$ (d, $\left.J=2.2 \mathrm{~Hz}, 2 \mathrm{H}\right), 6.65$ (t, $\left.J=2.2 \mathrm{~Hz}, 1 \mathrm{H}\right), 4.58$ (hept, $\left.J=6.1 \mathrm{~Hz}, 2 \mathrm{H}\right), 4.41$ (s, $2 \mathrm{H}), 1.35(\mathrm{~d}, J=6.0 \mathrm{~Hz}, 12 \mathrm{H})$.

${ }^{13} \mathrm{C}$ NMR (126 MHz, Chloroform-d) $\delta 191.2,159.5,135.9,109.5,108.4,70.5,31.2,22.1$.

IR (Neat): 2976, 1700, 1599, 1587, 1315, 1298, 1184, 1157, $1112 \mathrm{~cm}^{-1}$.

HRMS (ESI) $m / z$ calculated for $\mathrm{C}_{14} \mathrm{H}_{20} \mathrm{BrO}_{3}{ }^{+}\left([\mathrm{M}+\mathrm{H}]^{+}\right)$315.0590, found 315.0604.

${ }^{1} \mathrm{H}$ NMR, $401 \mathrm{MHz}$, Chloroform- $d$, Compound S14

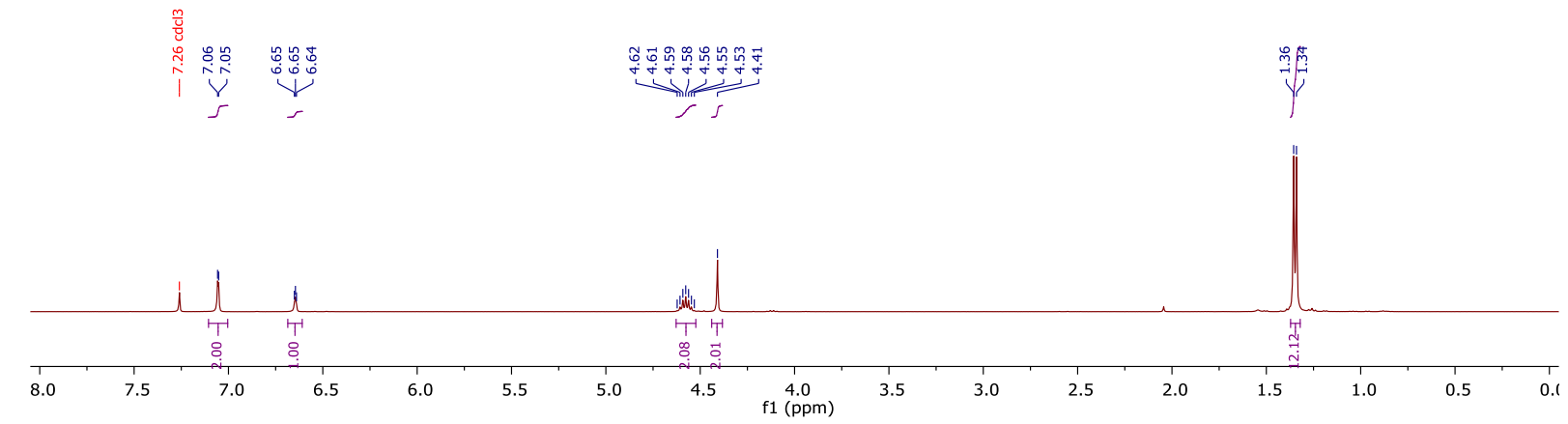

${ }^{13} \mathrm{C}$ NMR, $126 \mathrm{MHz}$, Chloroform- $d$, Compound S14

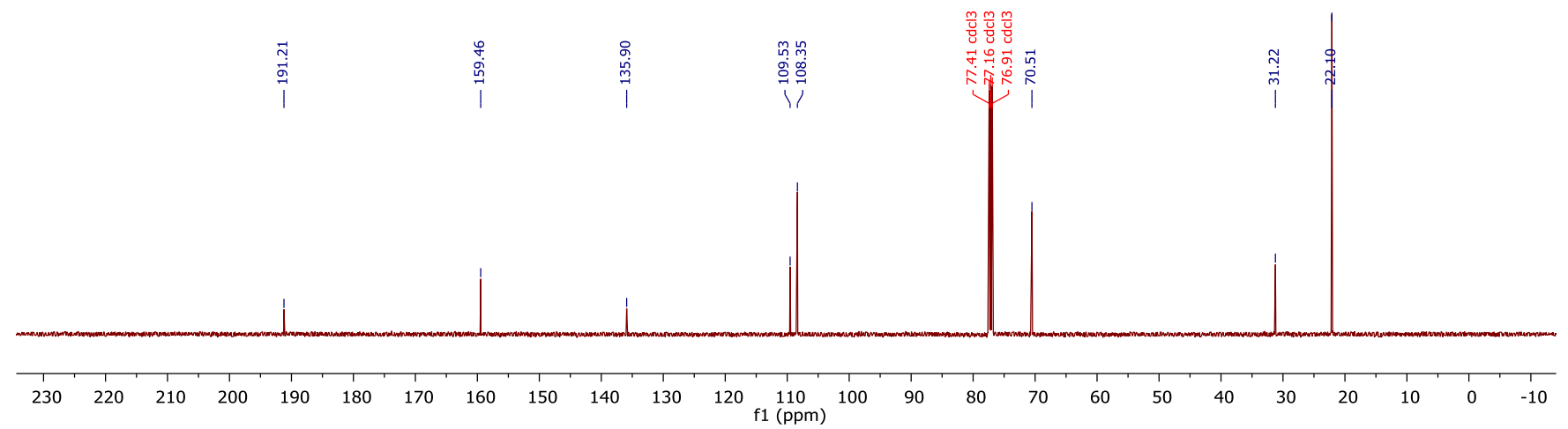




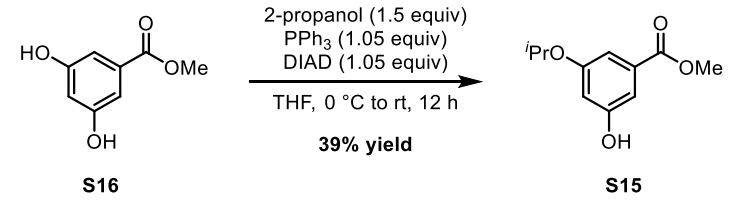

\section{S15 - methyl 3-hydroxy-5-isopropoxybenzoate}

The starting ester $\mathbf{S 1 6}$ (6.00 g, $35.7 \mathrm{mmol})$, 2-propanol (4.10 mL, $53.5 \mathrm{mmol}, 1.5$ equiv) and $\mathrm{PPh}_{3}(9.83 \mathrm{~g}, 37.5 \mathrm{mmol}, 1.05$ equiv) were added to a flame-dried $250-\mathrm{mL}$ round bottom flask charged with a stir bar under inert atmosphere. The starting materials were dissolved in THF $(120 \mathrm{~mL})$, and to the stirring solution was cooled to $0{ }^{\circ} \mathrm{C}$ in an ice bath. To the cold solution was added DIAD (7.38 mL, $37.5 \mathrm{mmol}, 1.05$ equiv) via syringe pump over 10 minutes. The reaction mixture was allowed to warm to room temperature over 12 hours, at which point the reaction was concentrated onto Celite and purified by column chromatography (5-30\% EtOAc in Hexanes) to afford the desired product $\mathbf{S 1 5}$ as a white solid (2.95 g, 39\% yield). The ${ }^{1} \mathrm{H}$ and ${ }^{13} \mathrm{C}$ NMR data for this compound were consistent with prior literature reports. ${ }^{5}$

${ }^{1} \mathrm{H}$ NMR (400 MHz, Chloroform- $d$ ) $\delta 7.15(\mathrm{dd}, J=2.3,1.3 \mathrm{~Hz}, 1 \mathrm{H}), 7.10(\mathrm{dd}, J=2.4,1.3 \mathrm{~Hz}, 1 \mathrm{H}), 6.59(\mathrm{t}, J=2.3 \mathrm{~Hz}, 1 \mathrm{H})$, $5.10(\mathrm{~s}, 1 \mathrm{H}), 4.57$ (hept, $J=6.1 \mathrm{~Hz}, 1 \mathrm{H}), 3.89(\mathrm{~s}, 3 \mathrm{H}), 1.33(\mathrm{~d}, J=6.0 \mathrm{~Hz}, 6 \mathrm{H})$.

${ }^{13} \mathrm{C}$ NMR (176 MHz, Chloroform- $\left.d\right) \delta 167.0,159.4,156.8,132.2,109.3,109.0,108.4,70.5,52.4,22.1$.

${ }^{1} \mathrm{H}$ NMR, 400 MHz, Chloroform-d, Compound S15
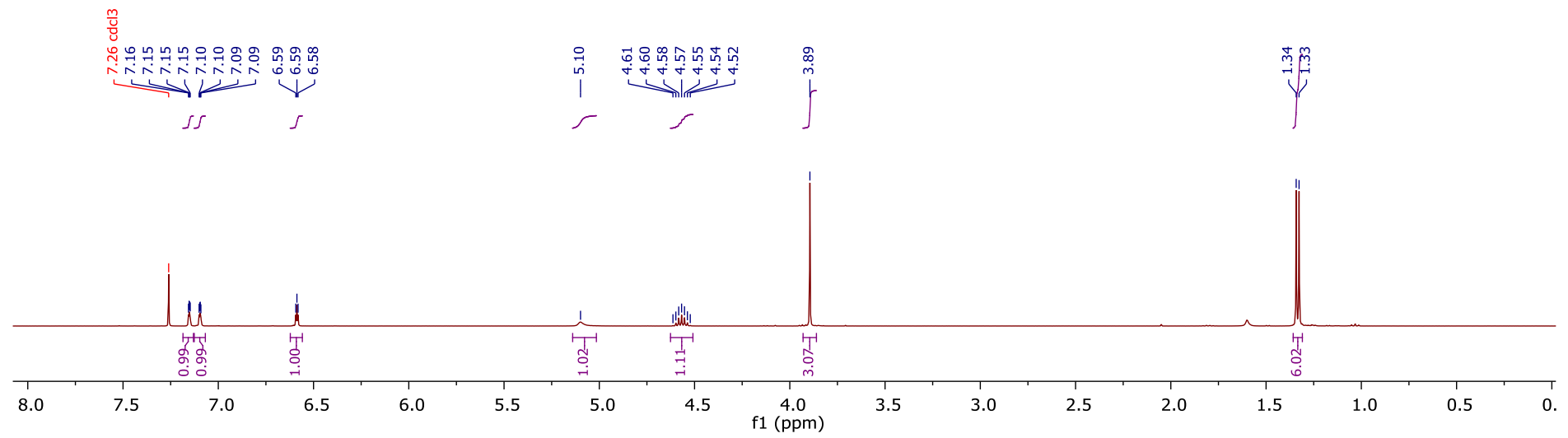

${ }^{13} \mathrm{C}$ NMR, $176 \mathrm{MHz}$, Chloroform- $d$, Compound $\mathbf{S 1 5}$

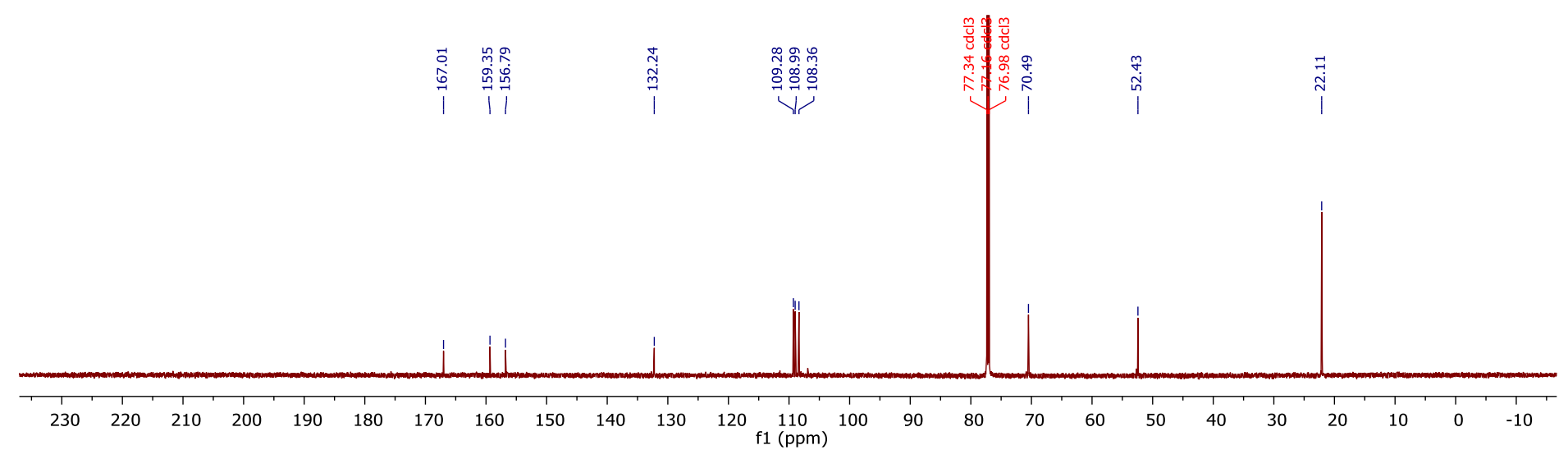




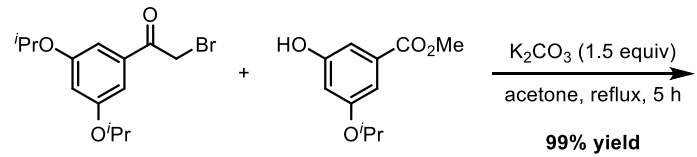

S14

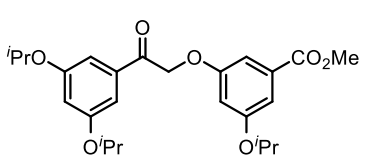

14B

\section{B - methyl 3-(2-(3,5-diisopropoxyphenyl)-2-oxoethoxy)-5-isopropoxybenzoate}

A solution of the starting phenol $\mathbf{S 1 5}$ (2.95 g, $14.0 \mathrm{mmol}, 1.0$ equiv) and bromide $\mathbf{S 1 4}$ (5.75 g, $18.2 \mathrm{mmol}, 1.30$ equiv) in acetone $(140 \mathrm{~mL})$ was prepared in a flame-dried, $500 \mathrm{~mL} \mathrm{rb}$ flask fitted with reflux condenser under inert atmosphere. To the stirring solution was added $\mathrm{K}_{2} \mathrm{CO}_{3}(2.91 \mathrm{~g}, 21.0 \mathrm{mmol}, 1.50$ equiv) in a single portion, and the reaction was heated to reflux for $5 \mathrm{~h}$. Upon cooling to rt, the crude reaction was concentrated under reduced pressure. The residue was dissolved in EtOAc and added to a sep. funnel containing DI $\mathrm{H}_{2} \mathrm{O}$. The layers were separated, and the organic layer was washed with brine, dried over $\mathrm{MgSO}_{4}$, and concentrated. The crude product was purified by flash column chromatography $(5 \%$ to $40 \%$ EtOAc in Hexanes over 10 column volumes) to afford the product 14B as a colorless oil (6.20 g, $13.9 \mathrm{mmol}, 99 \%$ yield $)$.

${ }^{1} \mathrm{H}$ NMR $(500 \mathrm{MHz}$, Chloroform-d) $\delta 7.21$ (dt, $J=2.3,1.1 \mathrm{~Hz}, 1 \mathrm{H}), 7.15(\mathrm{dt}, J=2.3,1.1 \mathrm{~Hz}, 1 \mathrm{H}), 7.06(\mathrm{~d}, J=1.9 \mathrm{~Hz}, 2 \mathrm{H})$, $6.71(\mathrm{~d}, J=2.5 \mathrm{~Hz}, 1 \mathrm{H}), 6.66(\mathrm{~d}, J=2.4 \mathrm{~Hz}, 1 \mathrm{H}), 5.25(\mathrm{~s}, 2 \mathrm{H}), 4.59(\mathrm{hept}, J=6.2 \mathrm{~Hz}, 3 \mathrm{H}), 3.89(\mathrm{~s}, 3 \mathrm{H}), 1.35(\mathrm{~d}, J=6.0$ $\mathrm{Hz}, 12 \mathrm{H}), 1.33(\mathrm{~d}, J=6.1 \mathrm{~Hz}, 6 \mathrm{H})$.

${ }^{13} \mathrm{C}$ NMR (126 MHz, Chloroform-d) $\delta 193.5,166.8,159.5,159.23,159.17,136.3,132.2,110.3,109.3,108.4,107.48$, $107.43,70.8,70.5,52.4,22.11,22.10$.

IR (Neat): 2976, 1717, 1700, 1590, 1457, 1437, 1296, 1156, $1112 \mathrm{~cm}^{-1}$.

HRMS (ESI) $m / z$ calculated for $\mathrm{C}_{25} \mathrm{H}_{33} \mathrm{O}_{7}{ }^{+}\left([\mathrm{M}+\mathrm{H}]^{+}\right) 445.2221$, found 445.2224

${ }^{1} \mathrm{H}$ NMR, $500 \mathrm{MHz}$, Chloroform- $d$, Compound 14B
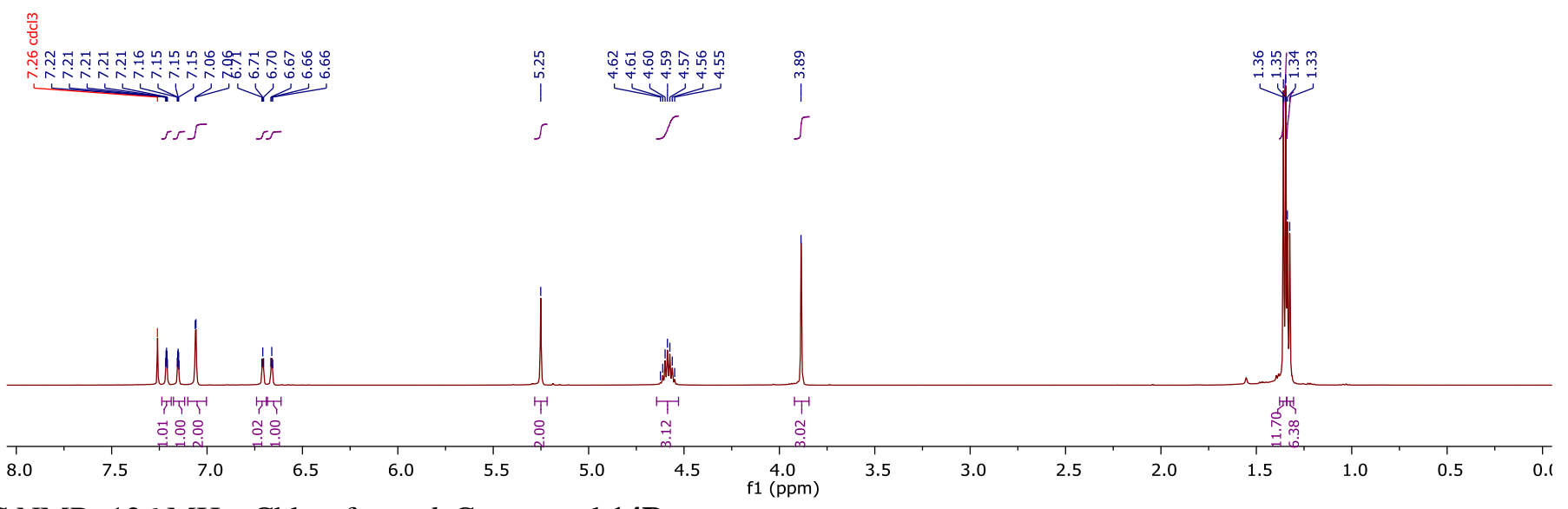

${ }^{13} \mathrm{C}$ NMR, $126 \mathrm{MHz}$, Chloroform- $d$, Compound 14B

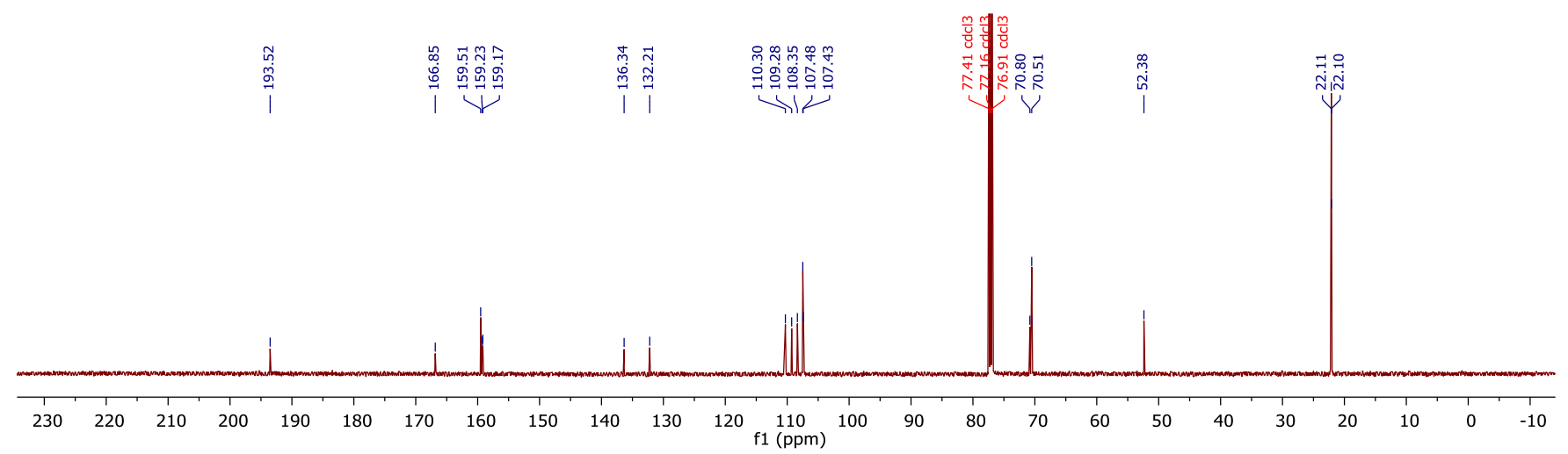



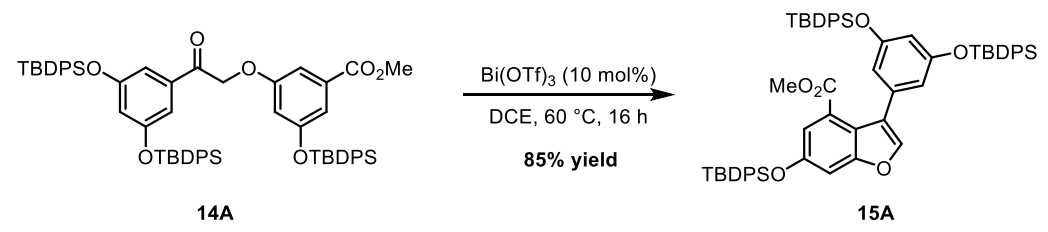

\section{A - methyl 3-(3,5-bis((tert-butyldiphenylsilyl)oxy)phenyl)-6-((tert-butyldiphenylsilyl)oxy)benzofuran-4- carboxylate}

The starting acetophenone 14A $(2.70 \mathrm{~g}, 2.61 \mathrm{mmol})$ was added to a flame-dried round bottom flask charged with a stir bar and fitted with a reflux condenser under inert atmosphere. The starting material was dissolved in 1,2-dichloroethane (DCE, $55 \mathrm{~mL})$ and $\mathrm{Bi}(\mathrm{OTf})_{3}(171 \mathrm{mg}, 0.261 \mathrm{mmol}, 10 \mathrm{~mol} \%)$ was added to the reaction under a stream of nitrogen. The reaction was heated at $60{ }^{\circ} \mathrm{C}$ for 16 hours; after cooling to room temperature, the reaction was filtered through a plug of Celite, eluting with DCM $(\sim 150 \mathrm{~mL})$. The filtrate was concentrated and purified by flash column chromatography $(0-10 \%$ EtOAc in Hexanes) to afford the desired product $(2.25 \mathrm{~g}, 85 \%$ yield $)$.

${ }^{1} \mathrm{H}$ NMR $(700 \mathrm{MHz}$, Chloroform- $d$ ) $\delta 7.73(\mathrm{~d}, J=8.0 \mathrm{~Hz}, 4 \mathrm{H}), 7.51(\mathrm{~d}, J=7.3 \mathrm{~Hz}, 8 \mathrm{H}), 7.44(\mathrm{t}, J=7.4 \mathrm{~Hz}, 2 \mathrm{H}), 7.38(\mathrm{t}, J$ $=7.7 \mathrm{~Hz}, 4 \mathrm{H}), 7.37-7.34(\mathrm{~m}, 5 \mathrm{H}), 7.24(\mathrm{t}, J=7.5 \mathrm{~Hz}, 8 \mathrm{H}), 7.13(\mathrm{~s}, 1 \mathrm{H}), 6.82(\mathrm{~d}, J=2.3 \mathrm{~Hz}, 1 \mathrm{H}), 6.32(\mathrm{~d}, J=2.2 \mathrm{~Hz}, 2 \mathrm{H})$, $6.12(\mathrm{t}, J=2.2 \mathrm{~Hz}, 1 \mathrm{H}), 3.11(\mathrm{~s}, 3 \mathrm{H}), 1.12(\mathrm{~s}, 9 \mathrm{H}), 0.99(\mathrm{~s}, 18 \mathrm{H})$.

${ }^{13} \mathrm{C}$ NMR (126 MHz, Chloroform- $d$ ) $\delta 167.4,156.4,155.9,153.0,135.64,135.60,134.8,132.8,132.4,130.2,129.9,128.1$, $127.8,125.2,122.6,118.9,118.3,113.6,106.1,51.2,26.7,26.6,19.6,19.5$.

IR (Neat): 2978, 1734, 1589, 1556, 1364, 1298, 1193, 1154, $1110 \mathrm{~cm}^{-1}$.

HRMS (ESI) $m / z$ calculated for $\mathrm{C}_{64} \mathrm{H}_{67} \mathrm{O}_{6} \mathrm{Si}_{3}{ }^{+}\left([\mathrm{M}+\mathrm{H}]^{+}\right)$1015.4240, found 1015.4238. 
${ }^{1} \mathrm{H}$ NMR, $700 \mathrm{MHz}$, Chloroform- $d$, Compound 15A
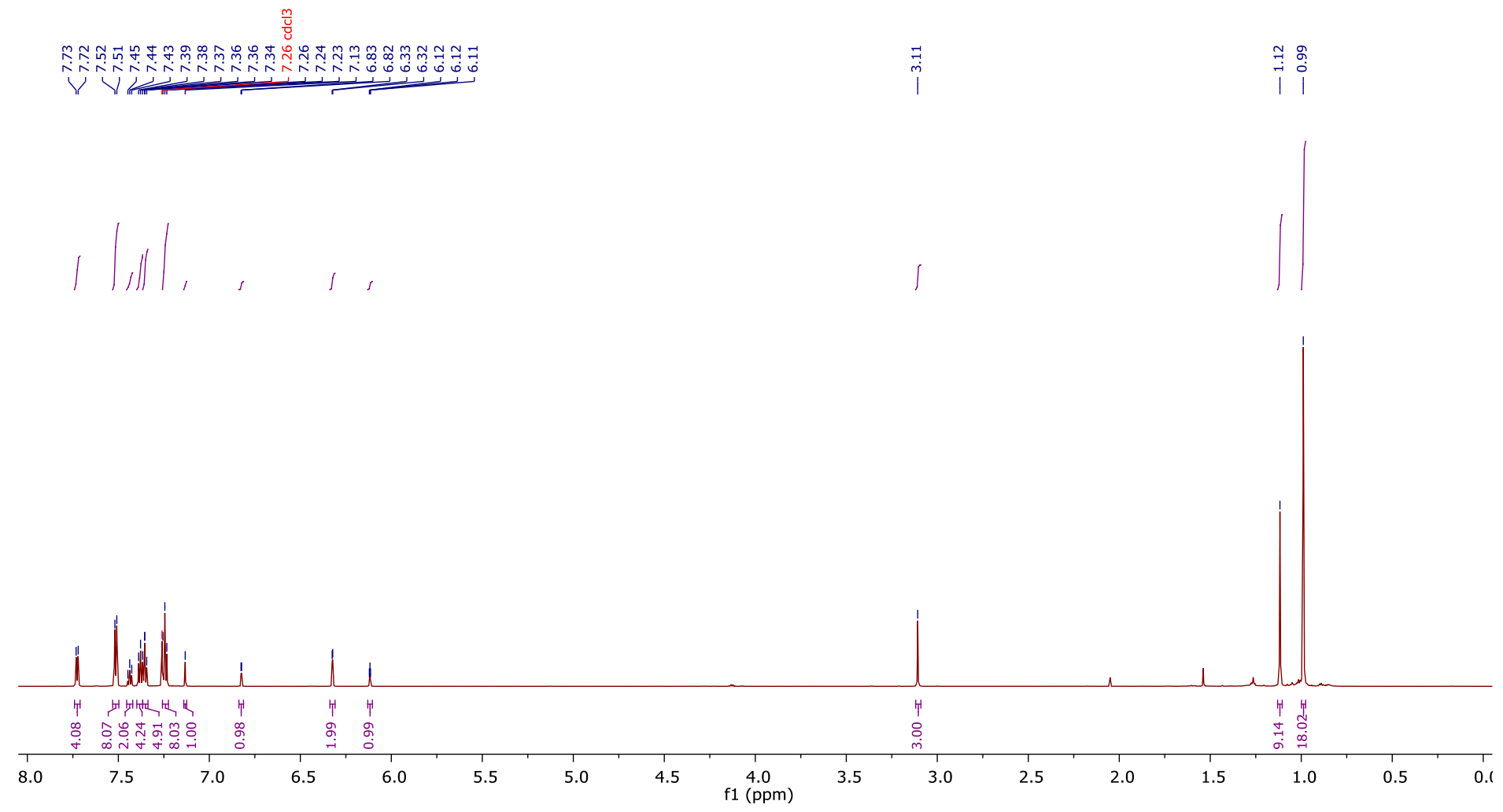

${ }^{13} \mathrm{C}$ NMR, $126 \mathrm{MHz}$, Chloroform- $d$, Compound 15A

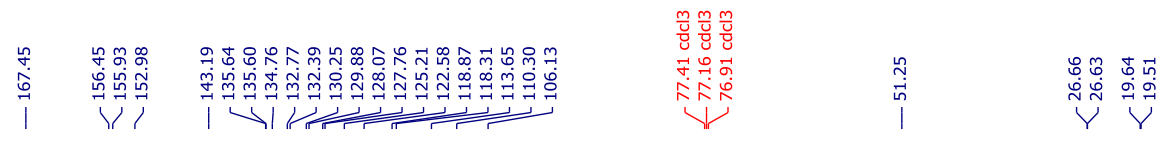

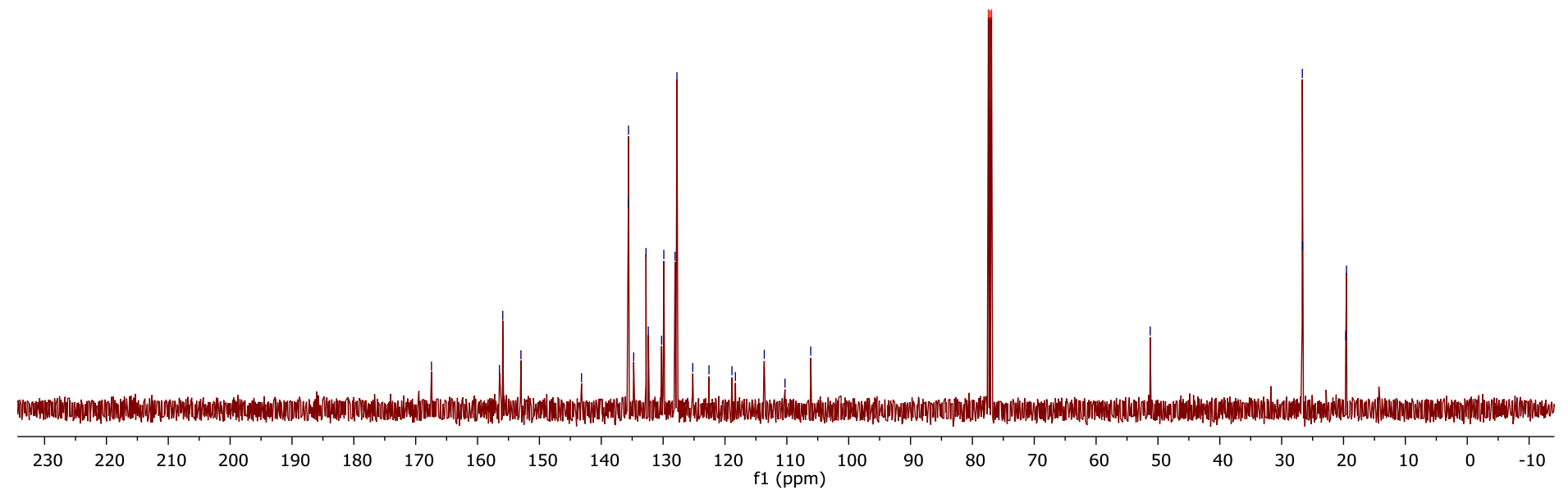




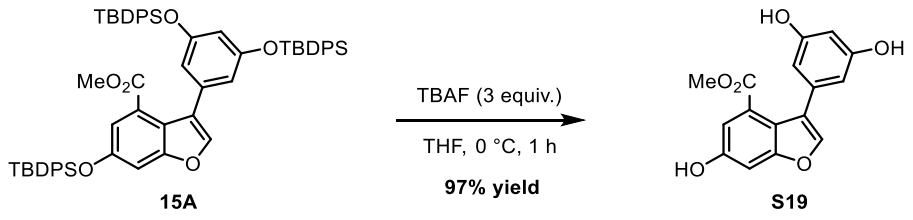

\section{S19 - methyl 3-(3,5-dihydroxyphenyl)-6-hydroxybenzofuran-4-carboxylate}

A solution of the starting benzofuran $\mathbf{1 5 A}$ (1.25 g, $1.23 \mathrm{mmol}, 1.0$ equiv.) in EtOAc (30 mL) was prepared in a flame-dried round bottom flask under inert atmosphere. The solution was cooled to $0{ }^{\circ} \mathrm{C}$ in an ice-bath, at which point a solution of TBAF (3.69 mL, $3.69 \mathrm{mmol}, 1.0 \mathrm{M}$ in THF, 3 equiv.) was added dropwise to the reaction mixture. After stirring for 1 hour at temperature, the reaction was quenched with $4 \mathrm{~N} \mathrm{HCl}(30 \mathrm{~mL})$, and this mixture was rapidly stirred for 5 minutes, at which point it was added to a separatory funnel containing a solution of saturated aqueous sodium bicarbonate $(\sim 100 \mathrm{~mL})$. The layers were separated, and the aqueous layer was extracted with additional EtOAc. The combined organic layers were dried over $\mathrm{MgSO}_{4}$ and concentrated under reduced pressure. The crude product was purified by flash column chromatography (25\% to $100 \%$ EtOAc in $\mathrm{CH}_{2} \mathrm{Cl}_{2}$ ) to afford the desired product $\mathbf{S 1 9}$ as an off-white solid (360 $\mathrm{mg}, 97 \%$ yield).

${ }^{1} \mathrm{H}$ NMR $\left(500 \mathrm{MHz}\right.$, Acetone- $\left.d_{6}\right) \delta 8.86(\mathrm{~s}, 1 \mathrm{H}), 8.30(\mathrm{~s}, 2 \mathrm{H}), 7.76(\mathrm{~s}, 1 \mathrm{H}), 7.20(\mathrm{~d}, J=1.8 \mathrm{~Hz}, 1 \mathrm{H}), 7.18(\mathrm{~d}, J=1.9 \mathrm{~Hz}$, $1 \mathrm{H}), 6.37$ (t, $J=2.1 \mathrm{~Hz}, 1 \mathrm{H}), 6.31(\mathrm{~d}, J=1.8 \mathrm{~Hz}, 2 \mathrm{H}), 3.34(\mathrm{~s}, 3 \mathrm{H})$.

${ }^{13} \mathrm{C}$ NMR $\left(126 \mathrm{MHz}\right.$, Acetone- $\left.d_{6}\right) \delta 168.1,159.3,158.0,156.0,143.5,136.0,127.1,124.0,118.4,114.1,107.9,102.3,102.0$, 51.5 .

IR (Neat): 3328 (br), 2927, 1684, 1613, 1440, 1341, 1266, 1157, 1135, $1002 \mathrm{~cm}^{-1}$.

HRMS (ESI) $m / z$ calculated for $\mathrm{C}_{16} \mathrm{H}_{13} \mathrm{O}_{6}{ }^{+}\left([\mathrm{M}+\mathrm{H}]^{+}\right)$301.0707, found 301.0697 . 
${ }^{1} \mathrm{H}$ NMR, $500 \mathrm{MHz}$, Acetone- $d_{6}$, Compound S19

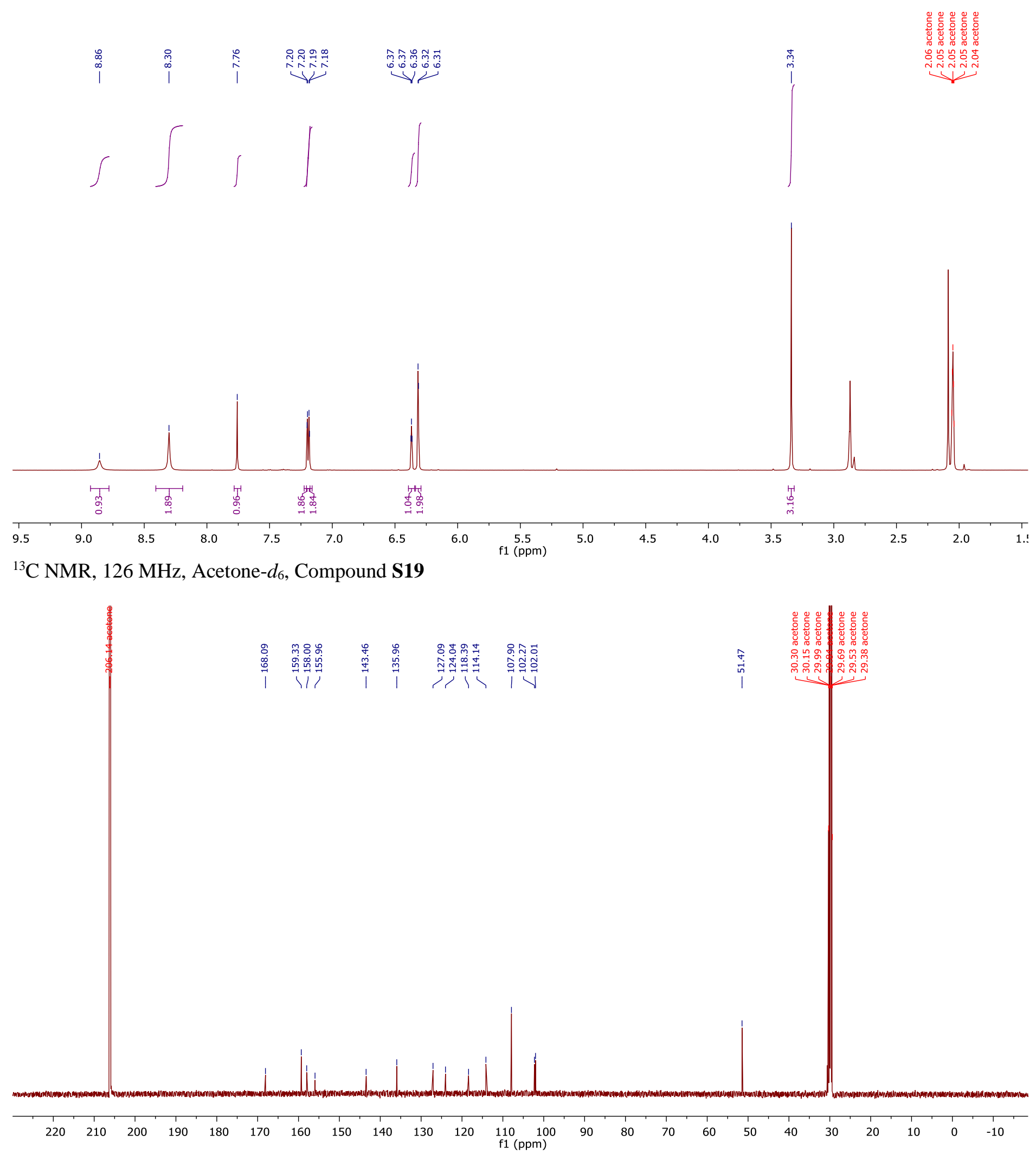




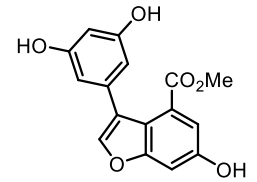

S19

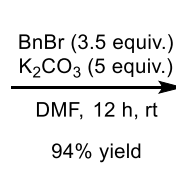

$94 \%$ yield

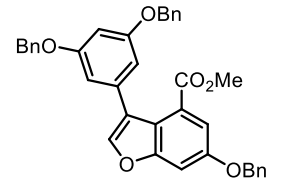

S17

S17 - methyl 6-(benzyloxy)-3-(3,5-bis(benzyloxy)phenyl)benzofuran-4-carboxylate

A flame-dried reaction vial was charged with the starting phenol S19 (100 $\mathrm{mg}, 0.333 \mathrm{mmol})$ and a stir bar under inert atmosphere. The starting material was dissolved in DMF $(3.0 \mathrm{~mL})$, and to the resulting solution was added potassium carbonate (5.0 equiv., $230 \mathrm{mg}, 1.67 \mathrm{mmol}$ ). The resulting reaction mixture was allowed to stir for $30 \mathrm{~min}$, at which point benzyl bromide ( 3.5 equiv., $1.4 \mathrm{~mL}, 1.17 \mathrm{mmol}$ ) was added dropwise via syringe. The resulting reaction mixture was allowed to stir for 12 hours, at which point the reaction was diluted with EtOAc $(\sim 15 \mathrm{~mL})$ and added to a separatory funnel containing water $(\sim 50 \mathrm{~mL})$. The layers were separated, and the aqueous layer was extracted with EtOAc $(2 \times 15 \mathrm{~mL})$, and the combined organic layers were washed with water $(3 \times 50 \mathrm{~mL})$, washed with brine $(\sim 50 \mathrm{~mL})$, dried over magnesium sulfate, and concentrated under reduced pressure. The crude material was purified via flash column chromatography $(0 \%$ to $40 \% \mathrm{EtOAc/Hexanes)}$ to afford the desired product $\mathbf{S 1 7}$ as a clear, colorless oil (178 $\mathrm{mg}, 94 \%$ yield).

${ }^{1} \mathrm{H}$ NMR $(700 \mathrm{MHz}$, Chloroform- $d$ ) $\delta 7.60(\mathrm{~s}, 1 \mathrm{H}), 7.46(\mathrm{~d}, J=7.6 \mathrm{~Hz}, 2 \mathrm{H}), 7.45(\mathrm{~d}, J=2.3 \mathrm{~Hz}, 1 \mathrm{H}), 7.43(\mathrm{~d}, J=7.2 \mathrm{~Hz}$, $4 \mathrm{H}), 7.39(\mathrm{~m}, 6 \mathrm{H}), 7.34(\mathrm{~m}, 3 \mathrm{H}), 7.27(\mathrm{~d}, J=2.3 \mathrm{~Hz}, 1 \mathrm{H}), 6.63(\mathrm{~d}, J=2.3 \mathrm{~Hz}, 1 \mathrm{H}), 6.59(\mathrm{~d}, J=2.2 \mathrm{~Hz}, 2 \mathrm{H}), 5.17(\mathrm{~s}, 2 \mathrm{H})$, $5.05(\mathrm{~s}, 4 \mathrm{H}), 3.29(\mathrm{~s}, 3 \mathrm{H})$.

${ }^{13} \mathrm{C}$ NMR $(176 \mathrm{MHz}$, Chloroform- $d$ ) $\delta 167.8,159.9,157.0,156.6,143.2,136.9,136.6,135.4,128.8,128.7,128.3,128.2$, $127.7,127.6,125.7,122.9,118.9,114.0,107.8,101.44,101.42,71.0,70.2,51.6$.

IR (Neat): 3024, 2927, 1684, 1610, 1424, 1339, 1268, 1159, 1135, $1002 \mathrm{~cm}^{-1}$.

HRMS (ESI) $m / z$ calculated for $\mathrm{C}_{37} \mathrm{H}_{31} \mathrm{O}_{6}{ }^{+}\left([\mathrm{M}+\mathrm{H}]^{+}\right)$571.2115, found 571.2111. 
${ }^{1} \mathrm{H}$ NMR, 700 MHz, Chloroform-d, Compound S17

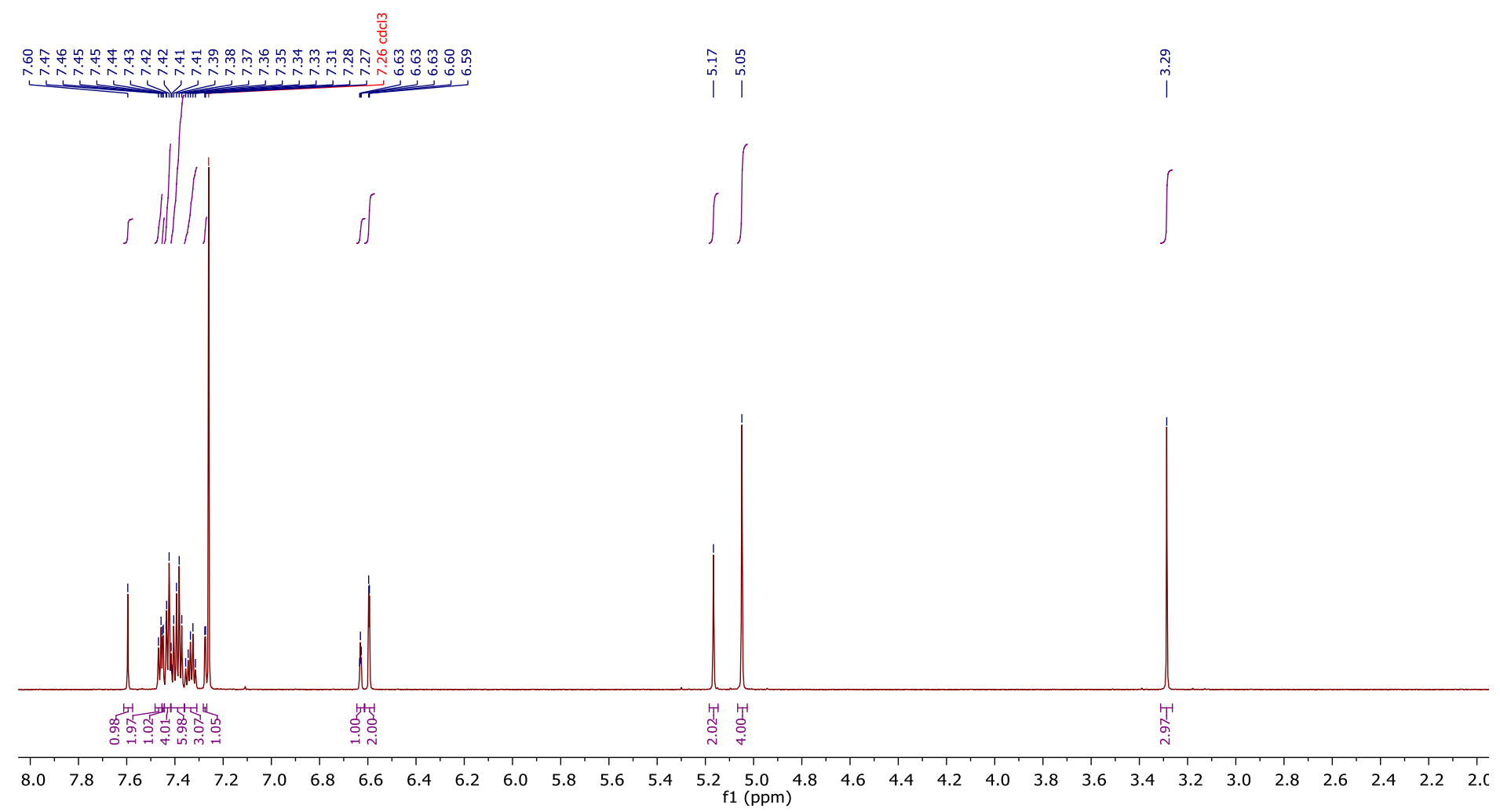

${ }^{13} \mathrm{C}$ NMR, $176 \mathrm{MHz}$, Chloroform- $d$, Compound $\mathbf{S 1 7}$
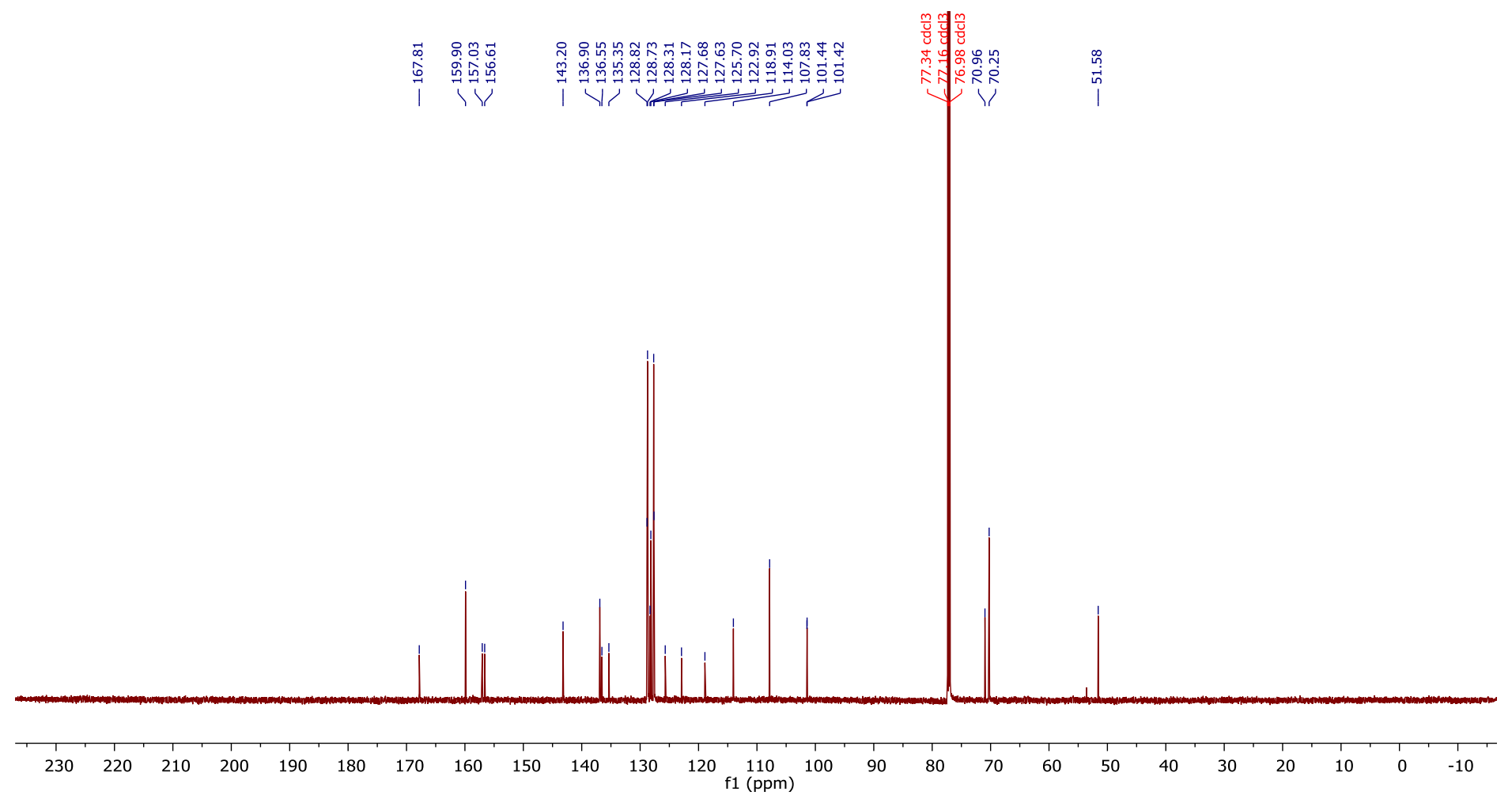


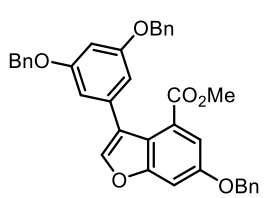

S17

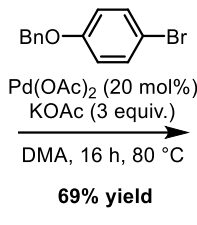

$69 \%$ yield

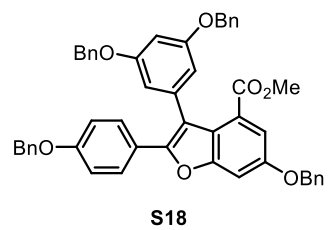

S18 - methyl 6-(benzyloxy)-2-(4-(benzyloxy)phenyl)-3-(3,5-bis(benzyloxy)phenyl)benzofuran-4-carboxylate

A flame-dried round botton flask was charged with the starting benzofuran S17 (590 $\mathrm{mg}, 1.03 \mathrm{mmol}$ ), 4benzyloxybromobenzene ( $816 \mathrm{mg}, 3.10 \mathrm{mmol}), \mathrm{Pd}(\mathrm{OAc})_{2}(46 \mathrm{mg}, 0.21 \mathrm{mmol})$, and KOAc (304 mg, $\left.3.10 \mathrm{mmol}\right)$ with a stir bar under inert atmosphere. The solids were dissolved/suspended in DMA $(18 \mathrm{~mL})$, and the reaction mixture was sparged with $\mathrm{N}_{2}$ for $>30 \mathrm{~min}$. The resulting reaction mixture was heated at $80{ }^{\circ} \mathrm{C}$ for 16 hours, at which point the reaction was diluted with EtOAc $(\sim 30 \mathrm{~mL})$ and filtered over Celite, eluting with EtOAc $(\sim 100 \mathrm{~mL})$. The filtrate was added to a separatory funnel containing water $(\sim 100 \mathrm{~mL})$. The layers were separated, and the organic layer was washed with water $(3 \mathrm{x} 100 \mathrm{~mL})$, washed with brine $(\sim 50 \mathrm{~mL})$, dried over magnesium sulfate, and concentrated under reduced pressure. The crude material was purified via flash column chromatography ( $4 \%$ to $32 \%, 8$ steps in $4 \%$ increments, $1.5 \mathrm{CV}$ ser step, polar phase $-2: 1$ $\mathrm{CH}_{2} \mathrm{Cl}_{2} / \mathrm{EtOAc}$, nonpolar phase - Hexanes) to afford the desired product as a clear, colorless oil (539 mg, 69\% yield).

${ }^{1} \mathrm{H}$ NMR $(500 \mathrm{MHz}$, Chloroform- $d$ ) $\delta 7.49-7.27(\mathrm{~m}, 24 \mathrm{H}), 6.87(\mathrm{~d}, J=8.5 \mathrm{~Hz}, 2 \mathrm{H}), 6.67(\mathrm{t}, J=2.4 \mathrm{~Hz}, 1 \mathrm{H}), 6.61(\mathrm{~d}, J=$ $2.2 \mathrm{~Hz}, 2 \mathrm{H}), 5.18$ (s, 2H), 5.07 (s, 2H), 5.03 (s, 4H), 3.17 (s, 3H).

${ }^{13} \mathrm{C}$ NMR (126 MHz, Chloroform- $d$ ) $\delta 167.9,160.3,159.0,156.2,155.3,152.0,136.89,136.85,136.82,136.7,128.80$, 128.76, 128.7, 128.4, 128.3, 128.2, 128.1, 127.63, 127.62, 127.58, 125.3, 123.3, 122.0, 115.9, 114.9, 113.6, 109.0, 102.1, $100.9,70.9,70.2,70.1,51.6$.

IR (Neat): 3024, 2927, 1684, 1610, 1424, 1339, 1268, 1159, 1135, $1002 \mathrm{~cm}^{-1}$.

HRMS (ESI) $m / z$ calculated for $\mathrm{C}_{50} \mathrm{H}_{41} \mathrm{O}_{7}{ }^{+}\left([\mathrm{M}+\mathrm{H}]^{+}\right)$753.2847, found 753.2830. 
${ }^{1} \mathrm{H}$ NMR, $500 \mathrm{MHz}$, Chloroform- $d$, Compound S18
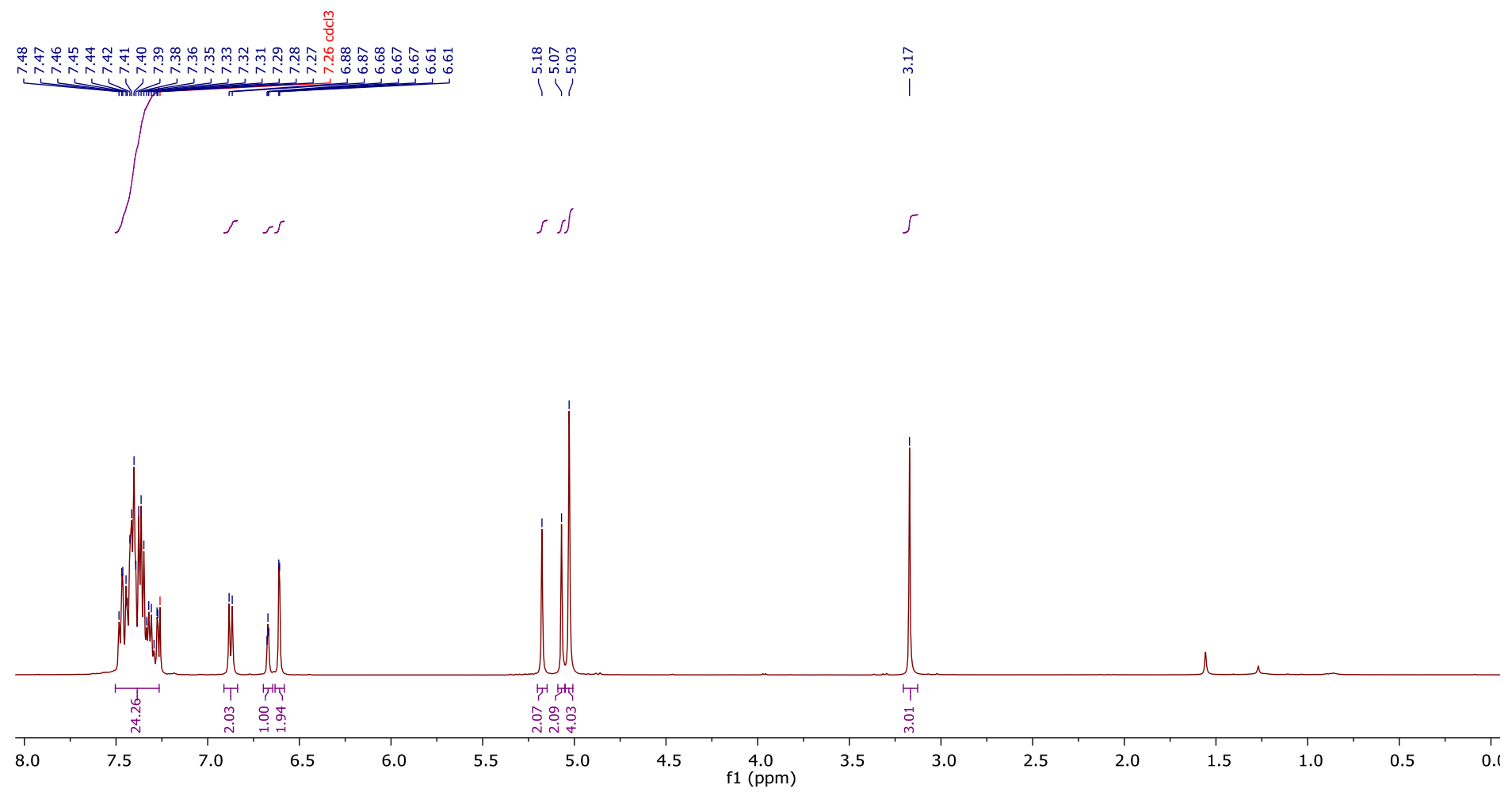

${ }^{13} \mathrm{C}$ NMR, $126 \mathrm{MHz}$, Chloroform- $d$, Compound S18

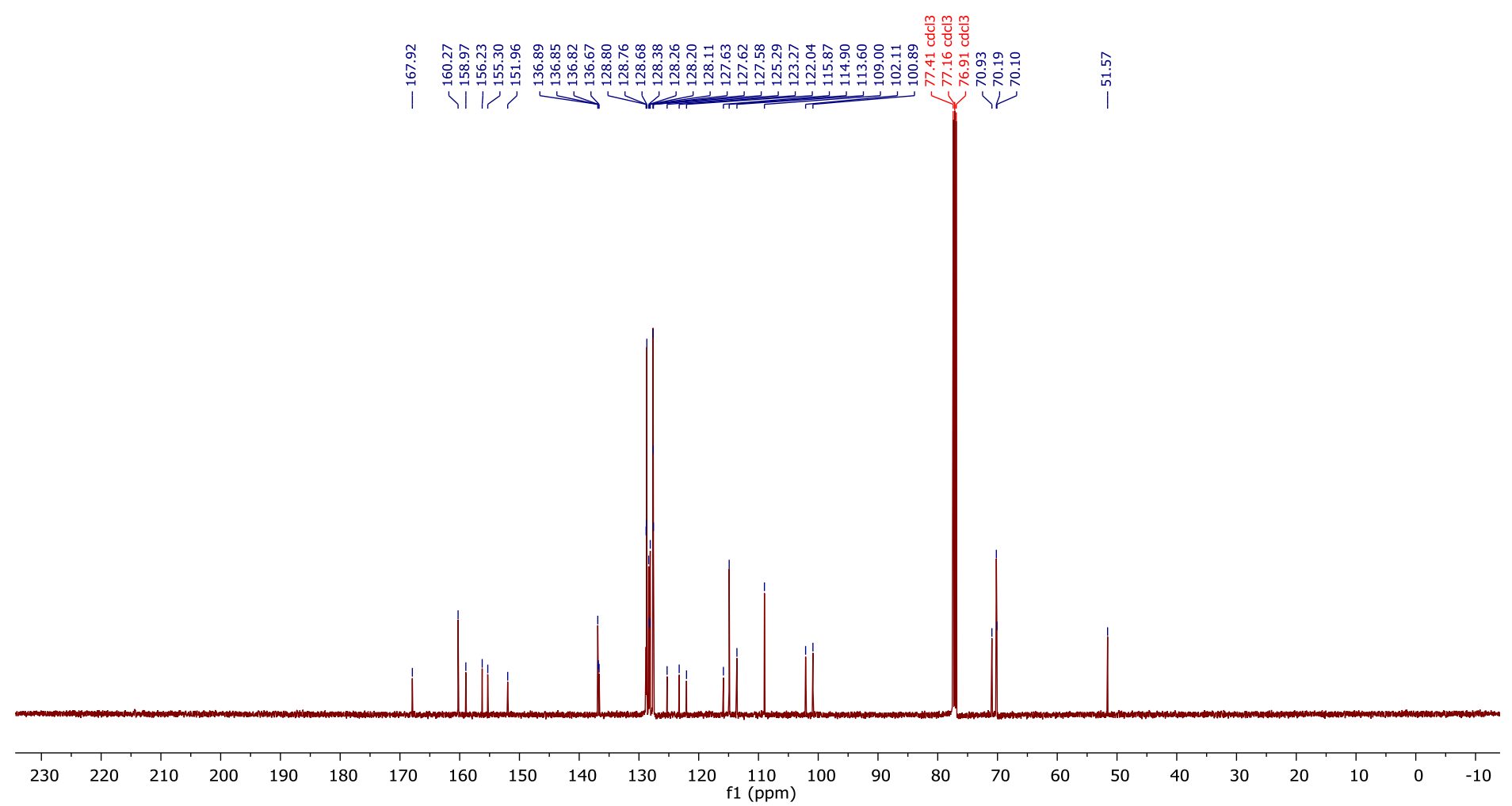



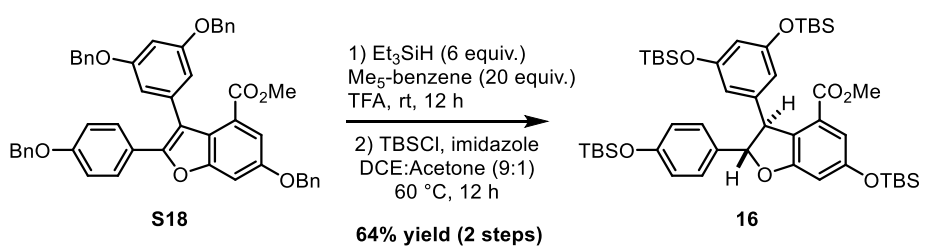

$64 \%$ yield ( 2 steps)

\section{6 - methyl (2R,3R)-3-(3,5-bis((tert-butyldimethylsilyl)oxy)phenyl)-6-((tert-butyldimethylsilyl)oxy)-2-(4-((tert- butyldimethylsilyl)oxy)phenyl)-2,3-dihydrobenzofuran-4-carboxylate}

Triethylsilane (197 $\mu \mathrm{l}, 1.24 \mathrm{mmol})$ was added to a stirred solution of $\mathbf{S 1 8}(155 \mathrm{mg}, 0.206 \mathrm{mmol})$ in trifluoroacetic acid (2 $\mathrm{mL}$ ). The reaction mixture was allowed to react at room temperature overnight. Upon reaction completion, the solution was cooled to $0^{\circ} \mathrm{C}$ and quenched with sat'd aq. $\mathrm{NaHCO}$. The quenched reaction was then extracted with ethyl acetate $(3 \times$ $10 \mathrm{~mL}$ ), and the combined organic layer was dried over $\mathrm{MgSO}_{4}$ and concentrated under reduced pressure. The crude residue was dissolved in minimal acetone $(\sim 200 \mu \mathrm{L})$, and the resulting solution was diluted with DCE $(1.8 \mathrm{~mL})$. Imidazole $(77 \mathrm{mg}$, $1.13 \mathrm{mmol})$ and $\mathrm{TBSCl}(155 \mathrm{mg}, 1.03 \mathrm{mmol})$ were added to the reaction solution, and the reaction mixture was heated at $60{ }^{\circ} \mathrm{C}$ for 12 hours. Upon completion of the reaction, the reaction was concentrated and purified by flash column chromatography (0 to $10 \%$ EtOAc/Hexanes) to afford the desired product as a colorless oil (111 mg, $64 \%$ yield). See page $\mathrm{S} 32$ for ${ }^{1} \mathrm{H}$ and ${ }^{13} \mathrm{C}$ NMR spectra for compound $\mathbf{1 6}$.

${ }^{1} \mathrm{H}$ NMR $(700 \mathrm{MHz}$, Chloroform- $d$ ) $\delta 7.16(\mathrm{~d}, J=8.5 \mathrm{~Hz}, 2 \mathrm{H}), 7.05(\mathrm{~d}, J=2.2 \mathrm{~Hz}, 1 \mathrm{H}), 6.81(\mathrm{~d}, J=8.5 \mathrm{~Hz}, 2 \mathrm{H}), 6.66(\mathrm{~d}, J$ $=2.2 \mathrm{~Hz}, 1 \mathrm{H}), 6.21(\mathrm{t}, J=2.2 \mathrm{~Hz}, 1 \mathrm{H}), 6.13(\mathrm{~d}, J=2.2 \mathrm{~Hz}, 2 \mathrm{H}), 5.39(\mathrm{~d}, J=5.1 \mathrm{~Hz}, 1 \mathrm{H}), 4.71(\mathrm{~d}, J=5.1 \mathrm{~Hz}, 1 \mathrm{H}), 3.54(\mathrm{~s}$, $3 \mathrm{H}), 1.01(\mathrm{~s}, 9 \mathrm{H}), 0.99(\mathrm{~s}, 9 \mathrm{H}), 0.93(\mathrm{~s}, 18 \mathrm{H}), 0.25(\mathrm{~s}, 6 \mathrm{H}), 0.20(\mathrm{~s}, 6 \mathrm{H}), 0.12(\mathrm{~s}, 12 \mathrm{H})$.

${ }^{13} \mathrm{C}$ NMR (176 MHz, Chloroform- $d$ ) $\delta 166.1,162.1,156.8,155.8,146.4,134.3,128.2,126.9,123.6,120.4,114.6,112.5$, $110.7,106.3,93.3,57.4,51.6,25.85,25.83,18.41,18.39,18.35,-4.21,-4.25,-4.26,-4.28$.

IR (Neat): 2955, 2930, 2895, 2858, 1727, 1161, 1588, 1473, 1343, 1253, 1161, 1030, $833 \mathrm{~cm}^{-1}$.

HRMS (ESI) $m / z$ calculated for $\mathrm{C}_{46} \mathrm{H}_{75} \mathrm{O}_{7} \mathrm{Si}_{4}{ }^{+}\left([\mathrm{M}+\mathrm{H}]^{+}\right)$851.4584, found 851.4580. 


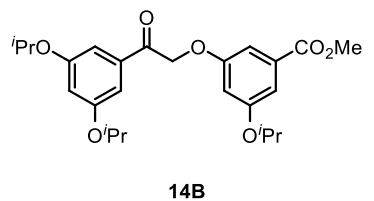

$\underset{\mathrm{DCE}, 60^{\circ} \mathrm{C}, 16 \mathrm{~h}}{\stackrel{\mathrm{BiTf})_{3}(10 \mathrm{~mol} \%)}{\longrightarrow}}$
$\mathbf{9 4 \%}$ yield

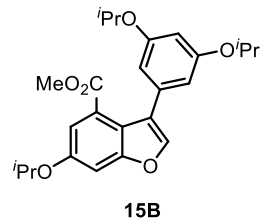

\section{B - methyl 3-(3,5-bis((tert-butyldiphenylsilyl)oxy)phenyl)-6-((tert-butyldiphenylsilyl)oxy)benzofuran-4- carboxylate}

The starting acetophenone 14B $(6.20 \mathrm{~g}, 13.9 \mathrm{mmol})$ was added to a flame-dried 500-mL round bottom flask charged with a stir bar and fitted with a reflux condenser under inert atmosphere. The starting material was dissolved in 1,2-dichloroethane (DCE, $200 \mathrm{~mL})$ and $\mathrm{Bi}(\mathrm{OTf})_{3}(915 \mathrm{mg}, 1.39 \mathrm{mmol}, 10 \mathrm{~mol} \%)$ was added to the reaction under a stream of nitrogen. The reaction was heated at $60{ }^{\circ} \mathrm{C}$ for 16 hours; after cooling to room temperature, the reaction was filtered through a plug of Celite, eluting with $\mathrm{CH}_{2} \mathrm{Cl}_{2}(\sim 200 \mathrm{~mL})$. The filtrate was concentrated and purified by flash column chromatography $(0-15 \%$ EtOAc in Hexanes) to afford the desired product 15B as a colorless oil (5.58 g, 94\% yield).

${ }^{1} \mathrm{H}$ NMR $(700 \mathrm{MHz}$, Chloroform-d) $\delta 7.58(\mathrm{~s}, 1 \mathrm{H}), 7.33(\mathrm{~d}, J=2.3 \mathrm{~Hz}, 1 \mathrm{H}), 7.20(\mathrm{~d}, J=2.3 \mathrm{~Hz}, 1 \mathrm{H}), 6.44(\mathrm{~d}, J=2.1 \mathrm{~Hz}$, $2 \mathrm{H}), 6.43(\mathrm{~d}, J=2.2 \mathrm{~Hz}, 1 \mathrm{H}), 4.62$ (hept, $J=6.0 \mathrm{~Hz}, 1 \mathrm{H}), 4.52$ (hept, $J=6.1 \mathrm{~Hz}, 2 \mathrm{H}), 3.32(\mathrm{~s}, 3 \mathrm{H}), 1.37(\mathrm{~d}, J=6.0 \mathrm{~Hz}, 6 \mathrm{H})$, $1.33(\mathrm{~d}, J=6.1 \mathrm{~Hz}, 12 \mathrm{H})$.

${ }^{13} \mathrm{C}$ NMR (176 MHz, Chloroform-d) $\delta 167.9,159.1,157.1,155.7,143.0,135.3,125.6,123.2,118.8,115.0,108.3,103.0$, $102.6,71.3,70.0,51.5,22.3,22.1$.

IR (Neat): 2976, 1734, 1718, 1592, 1436, 1317, 1248, 1154, 1136, $1104 \mathrm{~cm}^{-1}$.

HRMS (ESI) $m / z$ calculated for $\mathrm{C}_{25} \mathrm{H}_{31} \mathrm{O}_{6}{ }^{+}\left([\mathrm{M}+\mathrm{H}]^{+}\right) 427.2115$, found 427.2126. 
${ }^{1} \mathrm{H}$ NMR, $700 \mathrm{MHz}$, Chloroform- $d$, Compound 15B
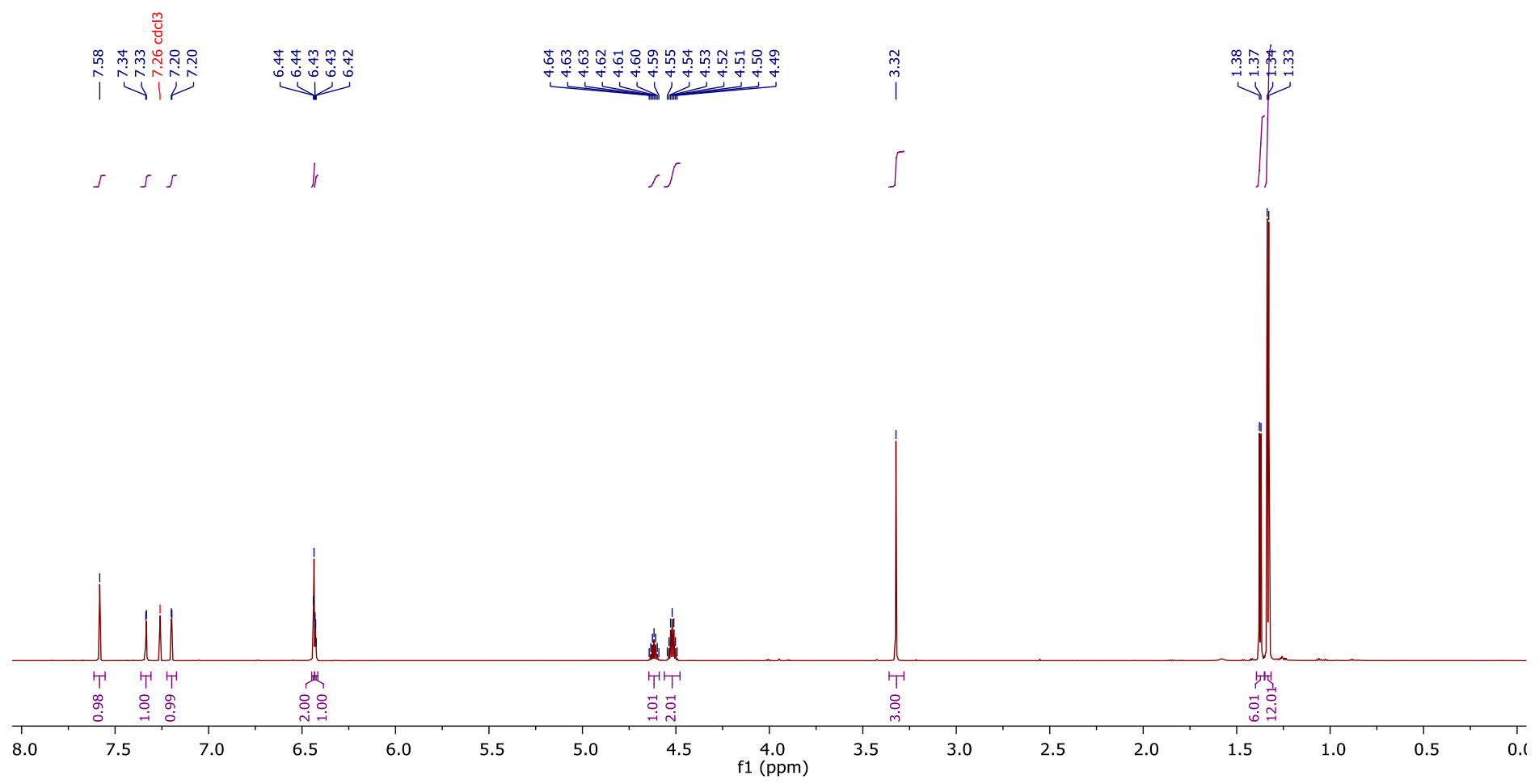

${ }^{13} \mathrm{C}$ NMR, $126 \mathrm{MHz}$, Chloroform- $d$, Compound 15B

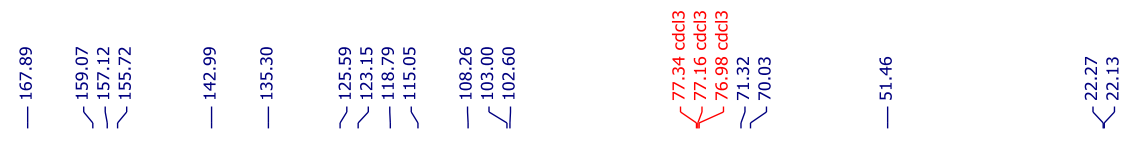

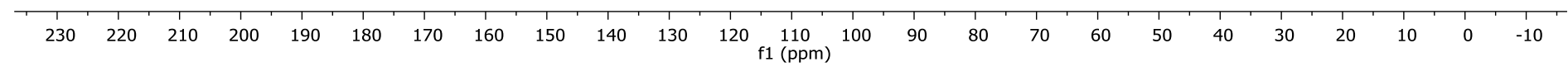




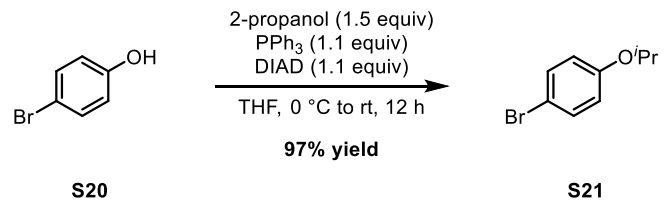

\section{S21 - 1-bromo-4-isopropoxybenzene}

The starting phenol S20 (5.00 g, $28.9 \mathrm{mmol})$, 2-propanol (3.31 mL, $43.4 \mathrm{mmol}, 1.5$ equiv) and $\mathrm{PPh}_{3}(8.34 \mathrm{~g}, 31.8 \mathrm{mmol}$, 1.1 equiv) were added to a flame-dried $250-\mathrm{mL}$ round bottom flask charged with a stir bar under inert atmosphere. The starting materials were dissolved in THF $(90 \mathrm{~mL})$, and to the stirring solution was cooled to $0{ }^{\circ} \mathrm{C}$ in an ice bath. To the cold solution was added diisopropyl azodicarboxylate (DIAD, $6.26 \mathrm{~mL}, 31.8 \mathrm{mmol}, 1.1$ equiv) via syringe pump over 10 minutes. The reaction mixture was allowed to warm to room temperature over 12 hours, at which point the reaction was concentrated onto Celite and purified by column chromatography $\left(0-15 \% \mathrm{CH}_{2} \mathrm{Cl}_{2}\right.$ in Hexanes) to afford the desired product $\mathbf{S 2 1}$ as a colorless oil (6.01 g, 97\% yield). The ${ }^{1} \mathrm{H}$ and ${ }^{13} \mathrm{C}$ NMR data for this compound were consistent with prior literature reports. ${ }^{6}$

${ }^{1} \mathrm{H}$ NMR (400 MHz, Chloroform- $d$ ) $\delta 7.35$ (d, $\left.J=9.0 \mathrm{~Hz}, 2 \mathrm{H}\right), 6.76(\mathrm{~d}, J=8.9 \mathrm{~Hz}, 2 \mathrm{H}), 4.49$ (hept, $\left.J=6.1 \mathrm{~Hz}, 1 \mathrm{H}\right), 1.32$ $(\mathrm{d}, J=6.1 \mathrm{~Hz}, 6 \mathrm{H})$.

${ }^{13} \mathrm{C}$ NMR (100 MHz, Chloroform- $d$ ) $\delta$ 157.1, 132.4, 117.8, 112.7, 70.4, 22.1.

${ }^{1} \mathrm{H}$ NMR, 400 MHz, Chloroform- $d$, Compound S21
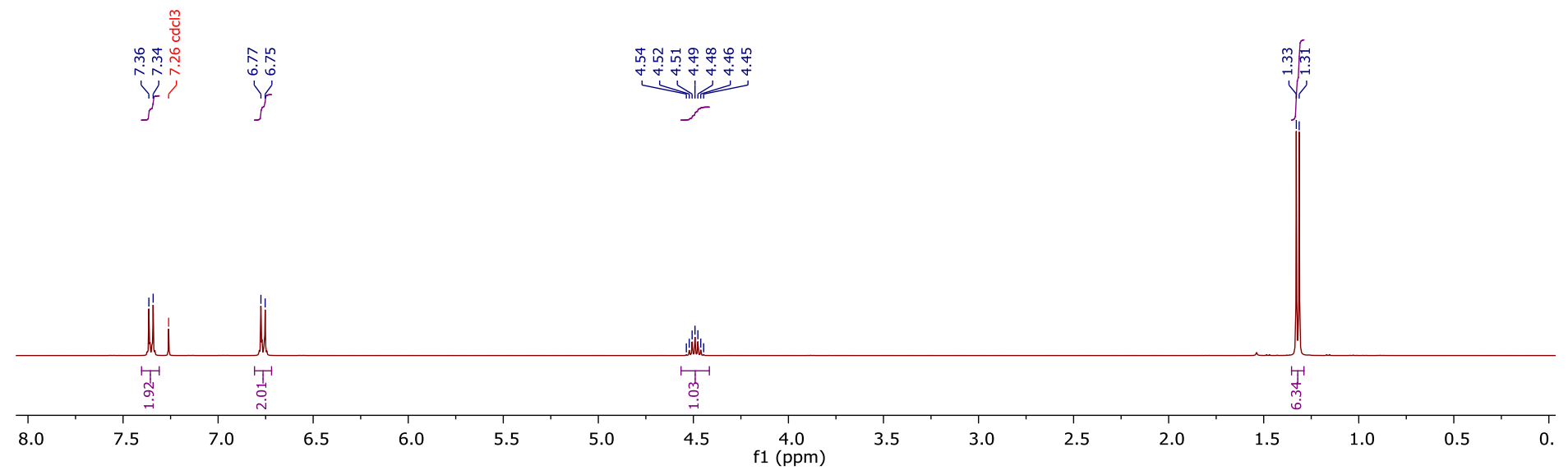

${ }^{13} \mathrm{C}$ NMR, $100 \mathrm{MHz}$, Chloroform- $d$, Compound S21

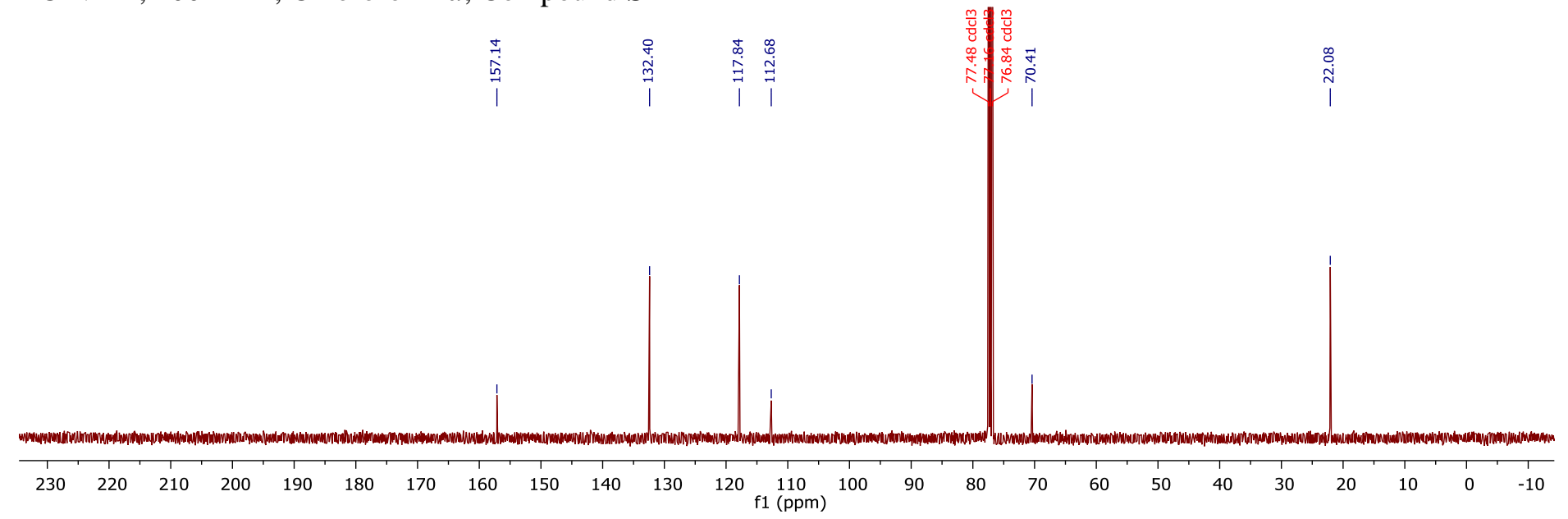



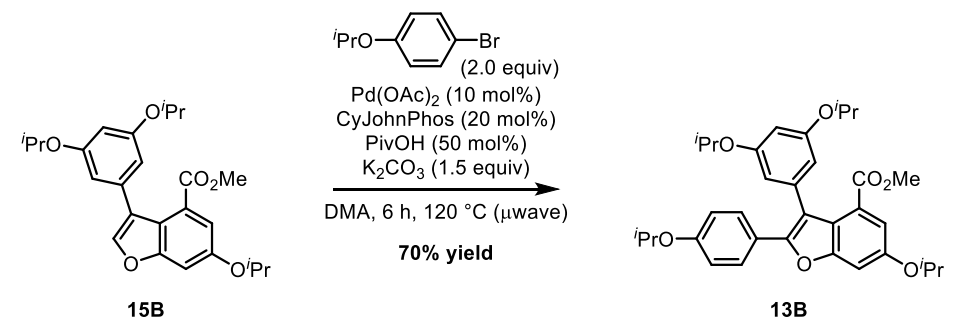

13B - methyl 3-(3,5-diisopropoxyphenyl)-6-isopropoxy-2-(4-isopropoxyphenyl)benzofuran-4-carboxylate

A flame-dried round heart-shaped flask was charged with the starting benzofuran $15 B$ (1.00 g, 2.34 mmol), 4isopropoxybromobenzene (S21, $1.01 \mathrm{~g}, 4.69 \mathrm{mmol}$ ), CyJohnPhos (164 mg, $0.469 \mathrm{mmol}$ ), pivalic acid (120 mg, $1.17 \mathrm{mmol}$ ), potassium carbonate $(486 \mathrm{mg}, 3.52 \mathrm{mmol})$, and $\mathrm{Pd}(\mathrm{OAc})_{2}(53 \mathrm{mg}, 0.234 \mathrm{mmol})$ with a stir bar under inert atmosphere. The solids were dissolved/suspended in degassed DMA (15 mL, degassed by Freeze-Pump-Thaw, 3 cycles), and the reaction mixture was degassed by Freeze-Pump-Thaw (3 cycles). The resulting reaction mixture was transferred to a $20-\mathrm{mL}$ microwave vial via cannula under a stream of $\mathrm{N}_{2}$. The microwave vial was capped under a stream of $\mathrm{N}_{2}$, and the reaction was irradiated at $120^{\circ} \mathrm{C}$ for 6 hours. Upon completion of microwave irradiation, the reaction was diluted with EtOAc $(\sim 30$ $\mathrm{mL})$ and filtered over Celite, eluting with EtOAc $(\sim 100 \mathrm{~mL})$. The filtrate was added to a separatory funnel containing water $(\sim 100 \mathrm{~mL})$. The layers were separated, and the organic layer was washed with water $(3 \mathrm{x} 100 \mathrm{~mL})$, washed with brine $(\sim 150$ $\mathrm{mL}$ ), dried over magnesium sulfate, and concentrated under reduced pressure. The crude material was purified via flash column chromatography ( $4 \%$ to $24 \%, 6$ steps in $4 \%$ increments, $2 \mathrm{CVs}$ per step, polar phase $-3: 1 \mathrm{CH}_{2} \mathrm{Cl}_{2} / \mathrm{EtOAc}$, nonpolar phase - Hexanes) to afford the desired product $\mathbf{1 3 B}$ as a clear, colorless oil (920 mg, 70\% yield).

${ }^{1} \mathrm{H}$ NMR $(500 \mathrm{MHz}$, Chloroform- $d) \delta 7.46(\mathrm{~d}, J=8.9 \mathrm{~Hz}, 2 \mathrm{H}), 7.25(\mathrm{~d}, J=2.3 \mathrm{~Hz}, 1 \mathrm{H}), 7.20(\mathrm{~d}, J=2.3 \mathrm{~Hz}, 1 \mathrm{H}), 6.78(\mathrm{~d}, J$ $=8.9 \mathrm{~Hz}, 2 \mathrm{H}), 6.46(\mathrm{t}, J=2.3 \mathrm{~Hz}, 1 \mathrm{H}), 6.45(\mathrm{~d}, J=2.3 \mathrm{~Hz}, 2 \mathrm{H}), 4.62$ (hept, $J=6.1 \mathrm{~Hz}, 1 \mathrm{H}), 4.53(\mathrm{hept}, J=6.1 \mathrm{~Hz}, 1 \mathrm{H})$, 4.48 (hept, $J=6.2 \mathrm{~Hz}, 2 \mathrm{H}), 3.25(\mathrm{~s}, 3 \mathrm{H}), 1.38(\mathrm{~d}, J=6.1 \mathrm{~Hz}, 6 \mathrm{H}), 1.32(\mathrm{~d}, J=6.1 \mathrm{~Hz}, 6 \mathrm{H}), 1.29(\mathrm{~d}, J=6.0 \mathrm{~Hz}, 12 \mathrm{H})$.

${ }^{13} \mathrm{C}$ NMR (126 MHz, Chloroform- $d$ ) $\delta 168.0,159.5,158.1,155.4,155.3,151.8,136.7,128.4,128.2,125.2,122.9,121.9$, 116.0, 115.7, 114.8, 114.6, 109.4, 109.3, 104.1, 104.0, 102.2, 102.0, 71.4, 71.3, 70.02, 70.01, 69.97, 69.93, 51.5, 22.2, 22.13, 22.07 .

IR (Neat): 2977, 1734, 1700, 1559, 1521, 1343, 1287, 1194, 1176, $1122 \mathrm{~cm}^{-1}$.

HRMS (ESI) $m / z$ calculated for $\mathrm{C}_{34} \mathrm{H}_{41} \mathrm{O}_{7}^{+}\left([\mathrm{M}+\mathrm{H}]^{+}\right)$561.2847, found 561.2849. 
${ }^{1} \mathrm{H}$ NMR, $500 \mathrm{MHz}$, Chloroform- $d$, Compound 13B
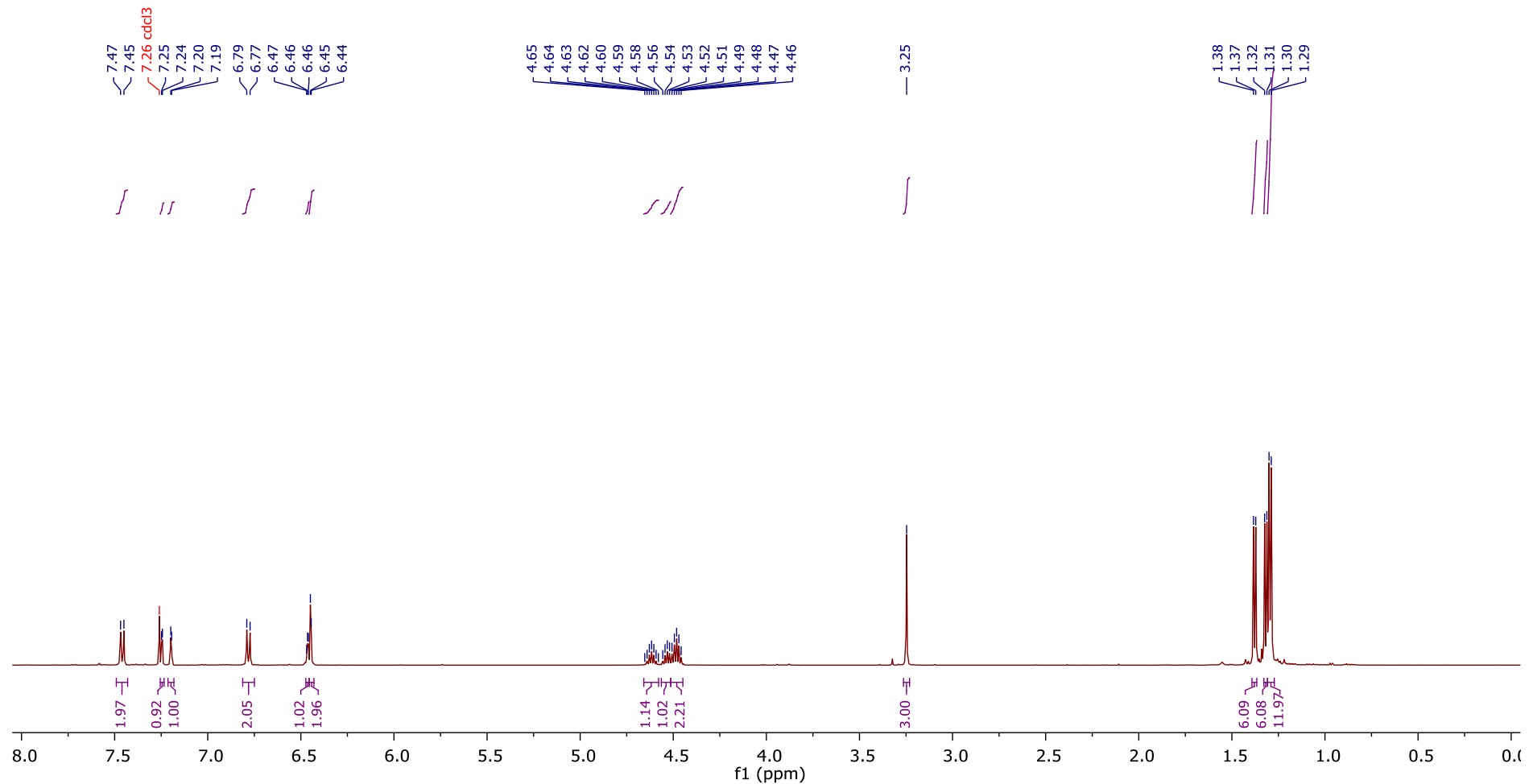

${ }^{13} \mathrm{C}$ NMR, $126 \mathrm{MHz}$, Chloroform- $d$, Compound 13B
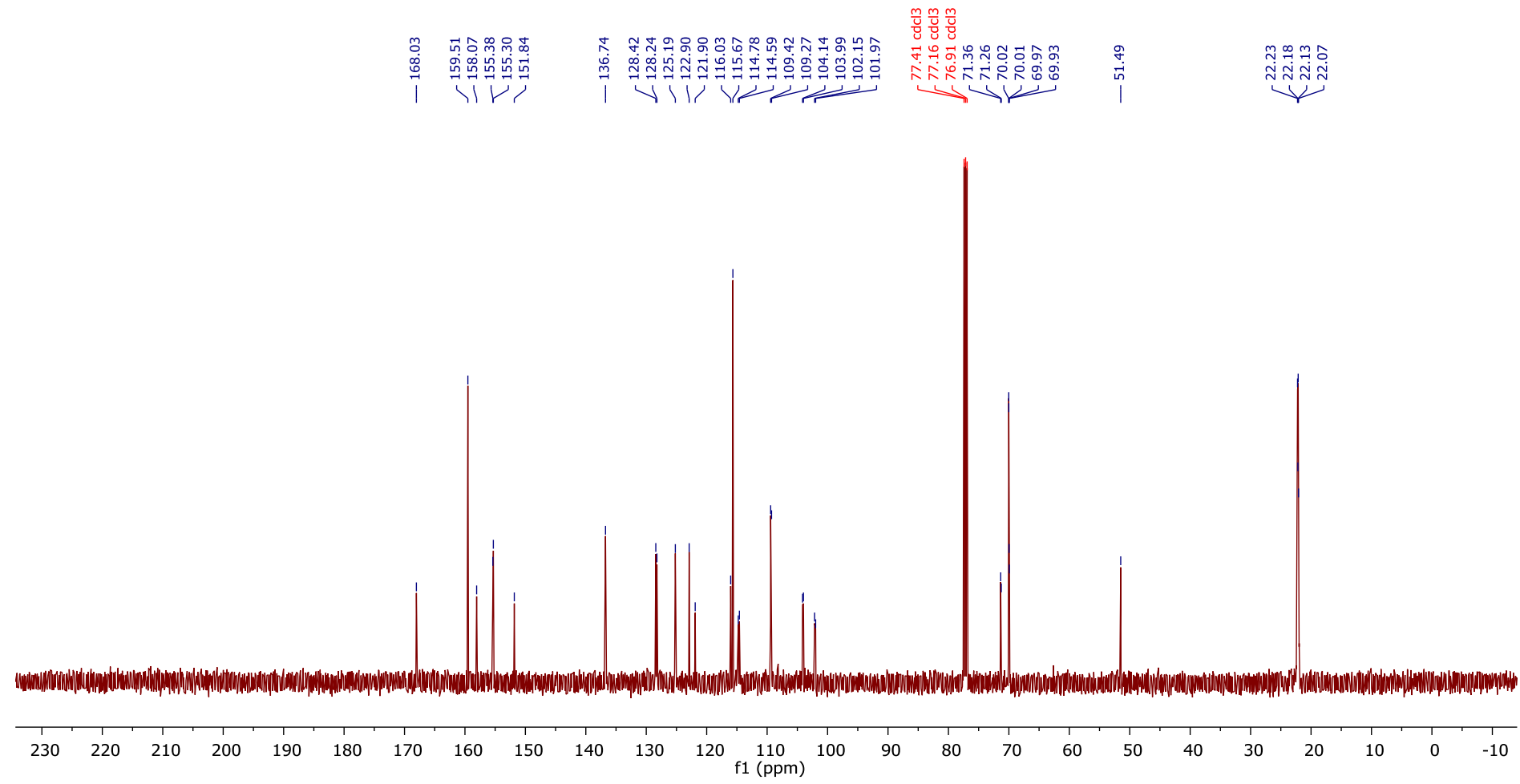

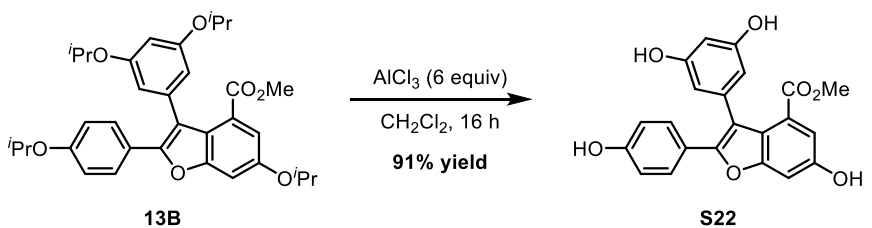

\section{S22 - methyl 3-(3,5-dihydroxyphenyl)-6-hydroxy-2-(4-hydroxyphenyl)benzofuran-4-carboxylate}

A flame-dried round bottom flask was charged with the starting benzofuran 13B (920 mg, $1.64 \mathrm{mmol})$ with a stir bar under inert atmosphere. The starting material was dissolved in $\mathrm{CH}_{2} \mathrm{Cl}_{2}(17 \mathrm{~mL})$ and $\mathrm{AlCl}_{3}(1.31 \mathrm{~g}, 9.85 \mathrm{mmol})$ was added to the reaction solution in a single portion under a stream of nitrogen. The reaction became yellow and then deep red as the deprotection occurred. After the reaction was complete, it was quenched with sat. aqueous $\mathrm{NH}_{4} \mathrm{Cl}(\sim 20 \mathrm{~mL})$ and transferred to a separatory funnel containing water $(\sim 100 \mathrm{~mL})$ and EtOAc $(\sim 100 \mathrm{~mL})$. The layers were separated, and the aqueous layer was washed with EtOAc $(3 \times 50 \mathrm{~mL})$. The combined organic layers were washed with brine $(\sim 200 \mathrm{~mL})$, dried over magnesium sulfate, and concentrated under reduced pressure. The crude material was purified via flash column chromatography ( $2 \%$ to $10 \%, 5$ steps in $2 \%$ increments, $2 \mathrm{CV}$ per step, polar phase $-\mathrm{MeOH}$, nonpolar phase $\left.-\mathrm{CH}_{2} \mathrm{Cl}_{2}\right)$ to afford the desired product as a white solid $(587 \mathrm{mg}, 91 \%$ yield).

${ }^{1} \mathrm{H}$ NMR $\left(500 \mathrm{MHz}\right.$, Acetone- $\left.d_{6}\right) \delta 8.49(\mathrm{~s}, 4 \mathrm{H}), 7.44(\mathrm{~d}, J=8.8 \mathrm{~Hz}, 2 \mathrm{H}), 7.18(\mathrm{~d}, J=2.2 \mathrm{~Hz}, 1 \mathrm{H}), 7.12(\mathrm{~d}, J=2.2 \mathrm{~Hz}, 1 \mathrm{H})$, $6.80(\mathrm{~d}, J=8.8 \mathrm{~Hz}, 2 \mathrm{H}), 6.42(\mathrm{t}, J=2.2 \mathrm{~Hz}, 1 \mathrm{H}), 6.30(\mathrm{~d}, J=2.2 \mathrm{~Hz}, 2 \mathrm{H}), 3.26(\mathrm{~s}, 3 \mathrm{H})$.

${ }^{13} \mathrm{C}$ NMR $\left(126 \mathrm{MHz}\right.$, Acetone- $\left.d_{6}\right) \delta 168.1,159.8,158.6,156.1,155.6,152.0,137.4,129.1,126.7,122.9,121.6,116.6,116.1$, $113.8,109.1,102.6,101.4,51.4$.

IR (Neat): 3326 (br), 2925, 1684, 1613, 1440, 1341, 1266, 1157, 1135, $1002 \mathrm{~cm}^{-1}$.

HRMS (ESI) $m / z$ calculated for $\mathrm{C}_{22} \mathrm{H}_{15} \mathrm{O}_{7}^{-}\left([\mathrm{M}-\mathrm{H}]^{-}\right)$391.0823, found 391.0830. 
${ }^{1} \mathrm{H}$ NMR, $500 \mathrm{MHz}$, Acetone- $d_{6}$, Compound S22

每

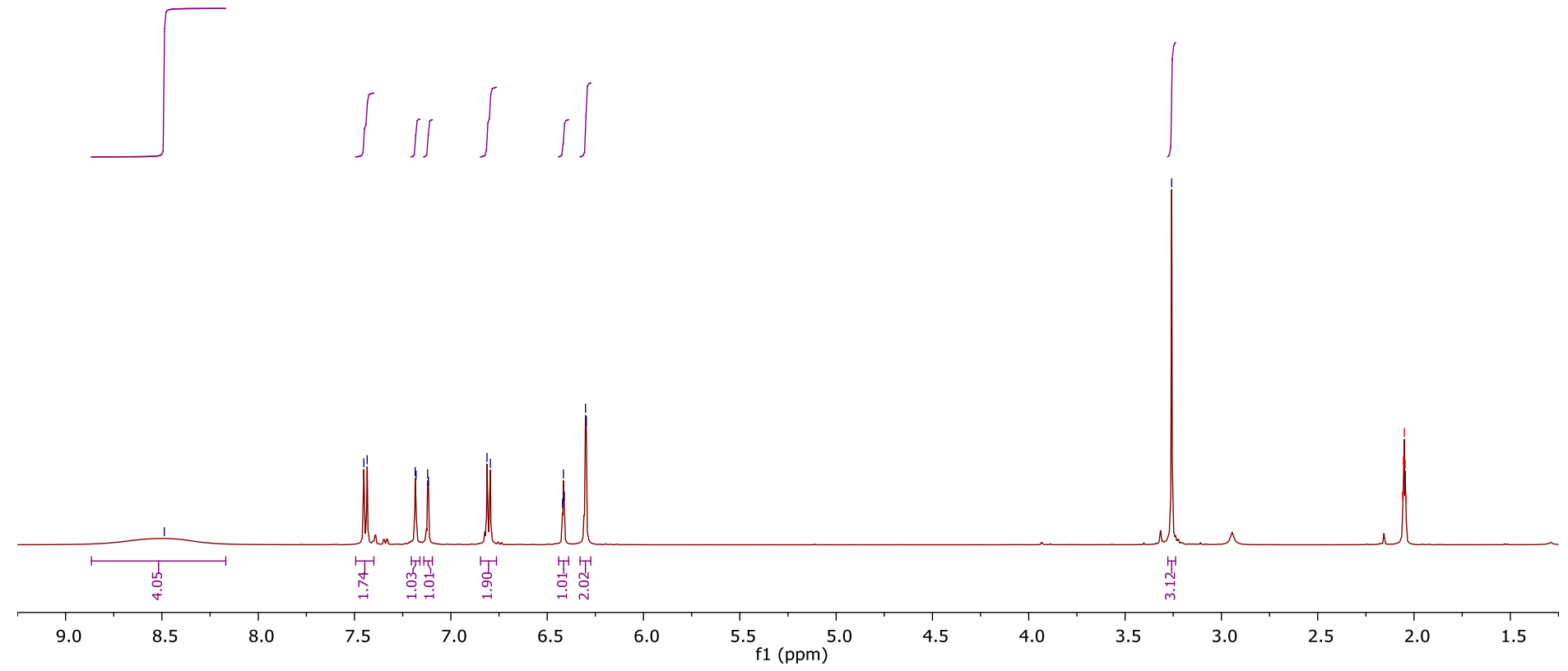

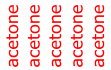

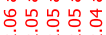

inivinis

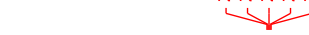

${ }^{13} \mathrm{C}$ NMR, $126 \mathrm{MHz}$, Acetone- $d_{6}$, Compound S22

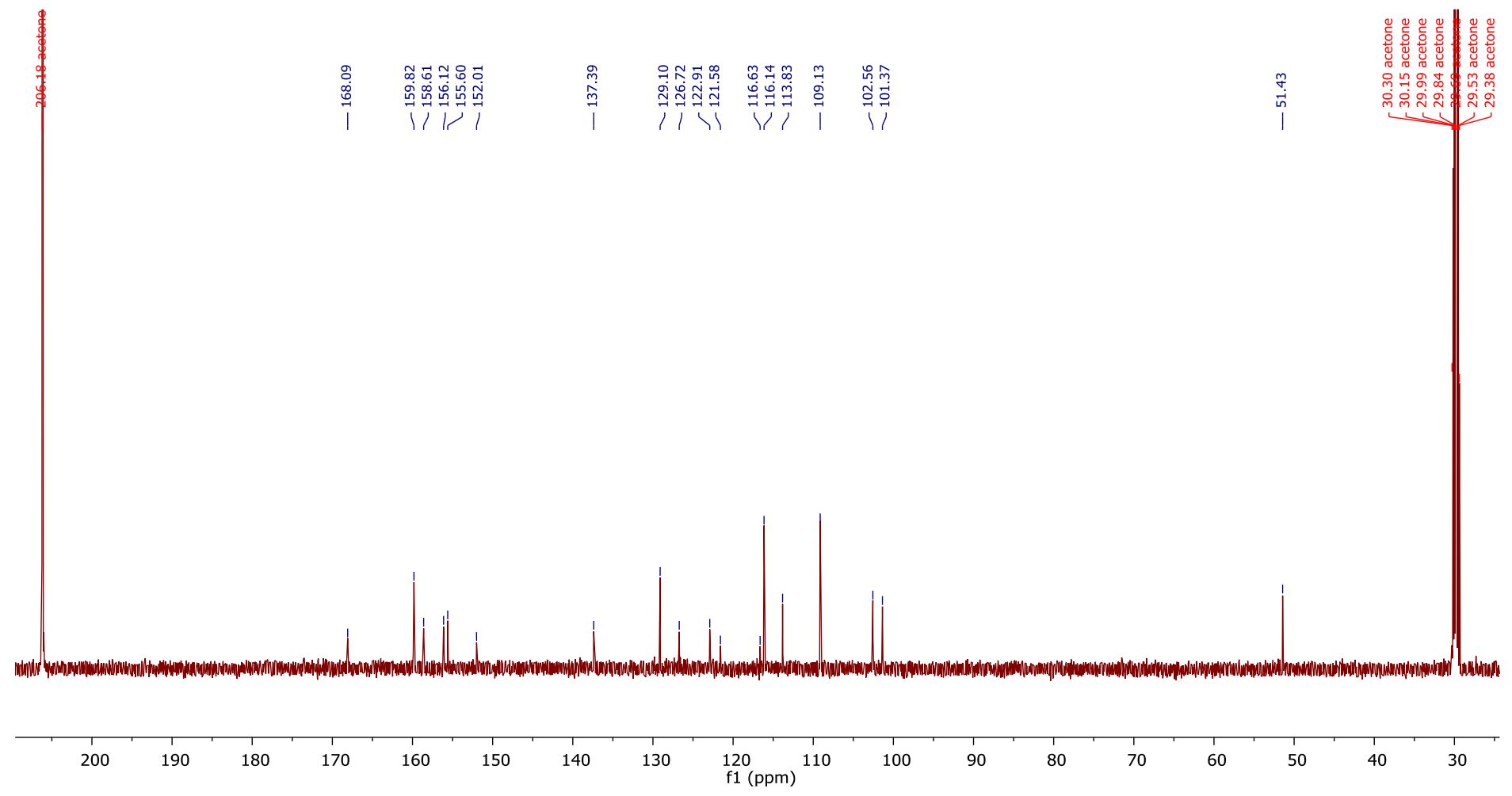



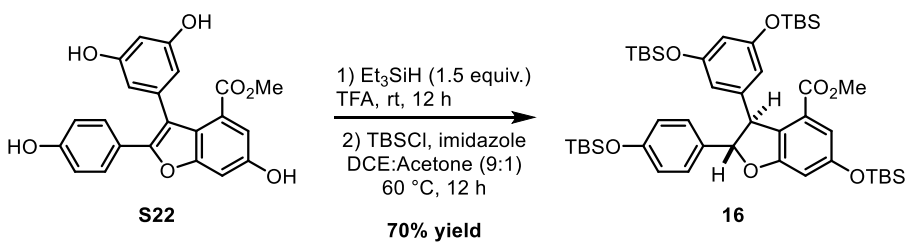

\section{6 - methyl (2R,3R)-3-(3,5-bis((tert-butyldimethylsilyl)oxy)phenyl)-6-((tert-butyldimethylsilyl)oxy)-2-(4-((tert- butyldimethylsilyl)oxy)phenyl)-2,3-dihydrobenzofuran-4-carboxylate}

Triethylsilane ( $305 \mu \mathrm{l}, 1.91 \mathrm{mmol})$ was added to a stirred solution of $\mathbf{S 2 2}(500 \mathrm{mg}, 1.27 \mathrm{mmol})$ in trifluoroacetic acid (10 $\mathrm{mL})$. The reaction mixture was stirred at room temperature overnight. Upon reaction completion, the solution was cooled to $0{ }^{\circ} \mathrm{C}$ and carefully quenched with sat'd aq. NaHCO3. The quenched reaction was then extracted with ethyl acetate $(3 \times$ $10 \mathrm{~mL}$ ), and the combined organic layer was dried over $\mathrm{MgSO}_{4}$ and concentrated under reduced pressure. The crude residue was dissolved in minimal acetone $(\sim 500 \mu \mathrm{L})$, and the resulting solution was diluted with DCE $(13 \mathrm{~mL})$. Imidazole $(476 \mathrm{mg}$, $6.99 \mathrm{mmol})$ and TBSCl $(957 \mathrm{mg}, 6.35 \mathrm{mmol})$ were added to the reaction solution, and the reaction mixture was heated at $60{ }^{\circ} \mathrm{C}$ for 12 hours. Upon completion of the reaction, the reaction was concentrated and purified by flash column chromatography (0 to 10\% EtOAc/Hexanes) to afford the desired product 16 as a colorless oil (758 $\mathrm{mg}, 70 \%$ yield).

${ }^{1} \mathrm{H}$ NMR $(700 \mathrm{MHz}$, Chloroform- $d$ ) $\delta 7.16(\mathrm{~d}, J=8.5 \mathrm{~Hz}, 2 \mathrm{H}), 7.05(\mathrm{~d}, J=2.2 \mathrm{~Hz}, 1 \mathrm{H}), 6.81(\mathrm{~d}, J=8.5 \mathrm{~Hz}, 2 \mathrm{H}), 6.66(\mathrm{~d}, J$ $=2.2 \mathrm{~Hz}, 1 \mathrm{H}), 6.21(\mathrm{t}, J=2.2 \mathrm{~Hz}, 1 \mathrm{H}), 6.13(\mathrm{~d}, J=2.2 \mathrm{~Hz}, 2 \mathrm{H}), 5.39(\mathrm{~d}, J=5.1 \mathrm{~Hz}, 1 \mathrm{H}), 4.71(\mathrm{~d}, J=5.1 \mathrm{~Hz}, 1 \mathrm{H}), 3.54(\mathrm{~s}$, $3 \mathrm{H}), 1.01$ (s, 9H), 0.99 (s, 9H), $0.93(\mathrm{~s}, 18 \mathrm{H}), 0.25(\mathrm{~s}, 6 \mathrm{H}), 0.20(\mathrm{~s}, 6 \mathrm{H}), 0.12(\mathrm{~s}, 12 \mathrm{H})$.

${ }^{13} \mathrm{C}$ NMR $(176 \mathrm{MHz}$, Chloroform- $d$ ) $\delta 166.1,162.1,156.8,155.8,146.4,134.3,128.2,126.9,123.6,120.4,114.6,112.5$, $110.7,106.3,93.3,57.4,51.6,25.85,25.83,18.41,18.39,18.35,-4.21,-4.25,-4.26,-4.28$.

IR (Neat): 2955, 2930, 2895, 2858, 1727, 1161, 1588, 1473, 1343, 1253, 1161, 1030, $833 \mathrm{~cm}^{-1}$.

HRMS (ESI) $m / z$ calculated for $\mathrm{C}_{46} \mathrm{H}_{75} \mathrm{O}_{7} \mathrm{Si}_{4}{ }^{+}\left([\mathrm{M}+\mathrm{H}]^{+}\right)$851.4584, found 851.4580 . 
${ }^{1} \mathrm{H}$ NMR, 700 MHz, Chloroform-d, Compound 16

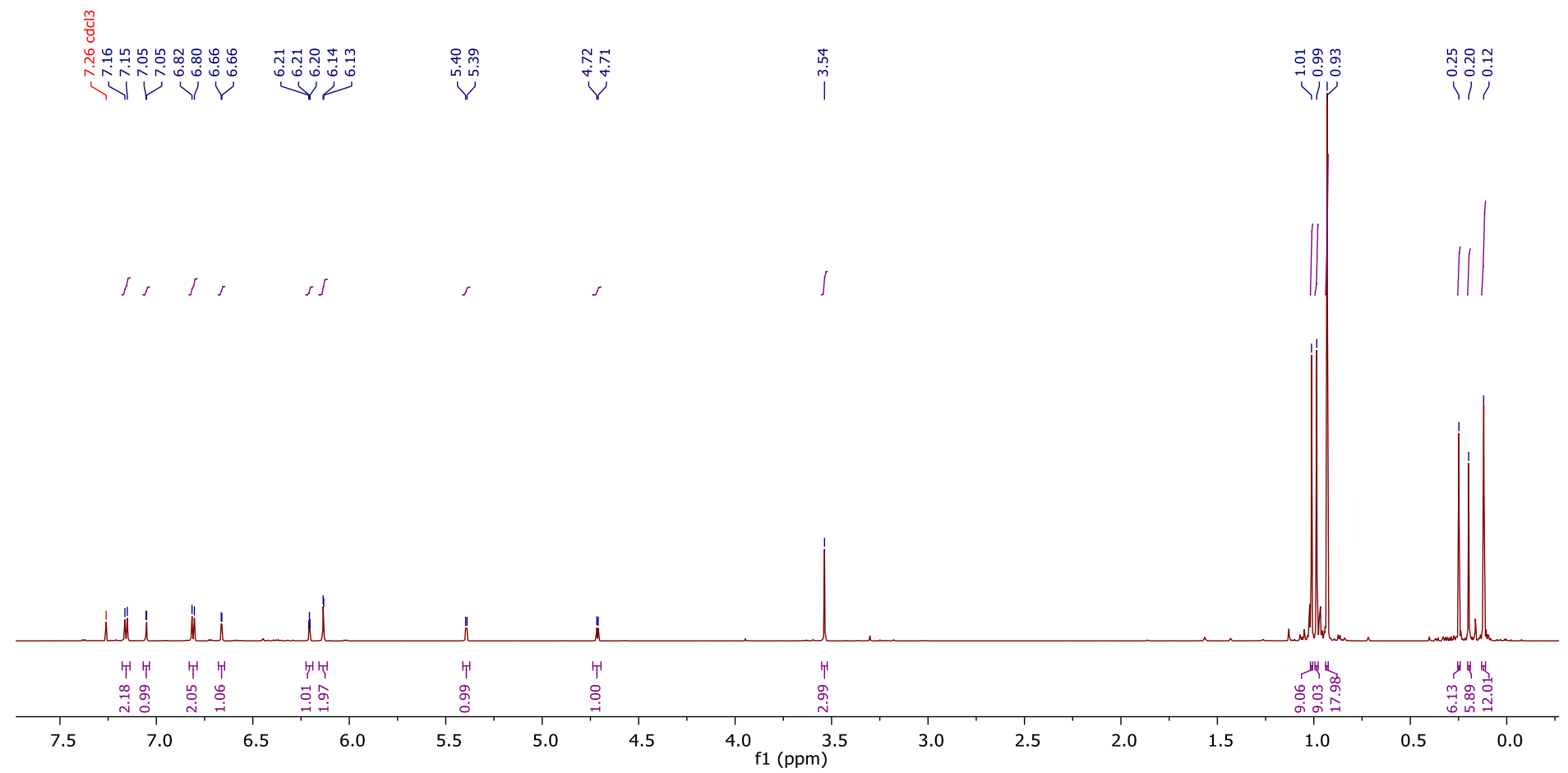

${ }^{13} \mathrm{C}$ NMR, $176 \mathrm{MHz}$, Chloroform- $d$, Compound 16

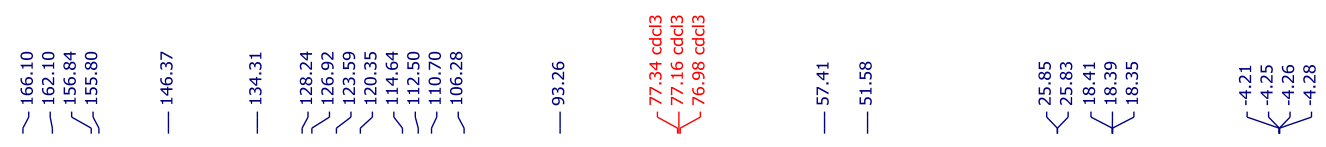

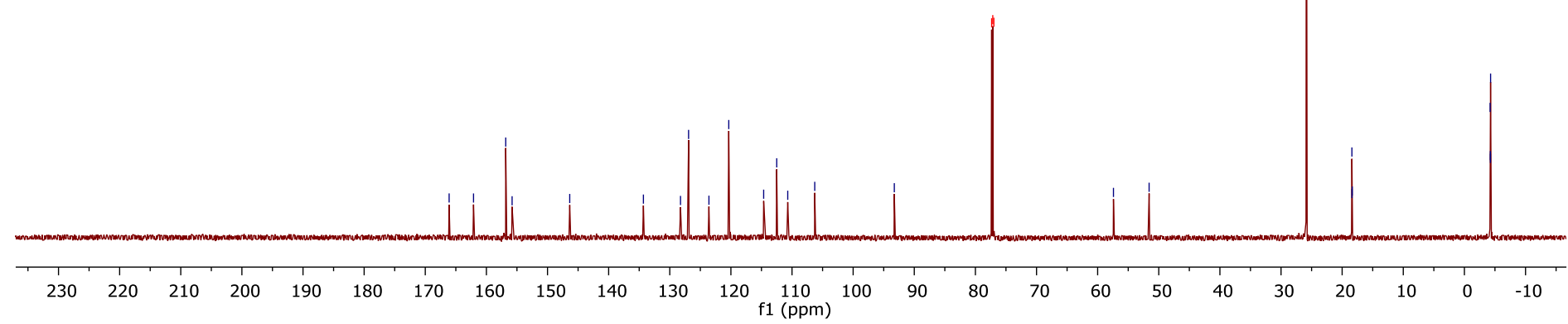



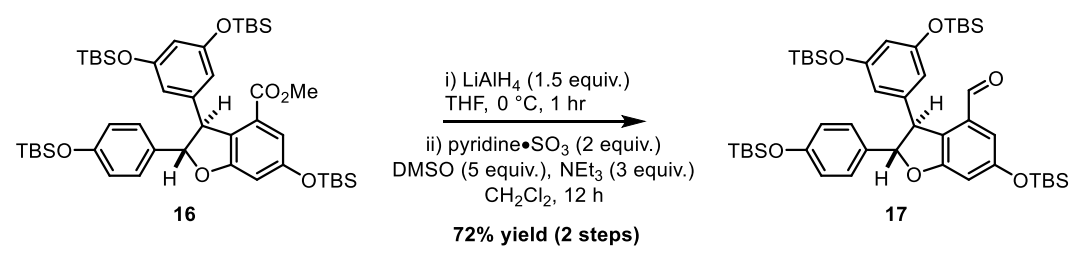

17 - (2R,3R)-3-(3,5-bis((tert-butyldimethylsilyl)oxy)phenyl)-6-((tert-butyldimethylsilyl)oxy)-2-(4-((tertbutyldimethylsilyl)oxy)phenyl)-2,3-dihydrobenzofuran-4-carbaldehyde

$\mathrm{LiAlH}_{4}$ (45 mg, $1.18 \mathrm{mmol}$ ) was added to a stirred solution of $\mathbf{1 6}(670 \mathrm{mg}, 0.787 \mathrm{mmol})$ in THF (15 mL) in an ice bath. The reaction mixture was allowed to react at $0{ }^{\circ} \mathrm{C}$ for 1 hour, at which point it was quenched with Glauber's salt. The precipitate was removed by filtration, and the filtrate was concentrated to afford the crude product, which was resuspended in $\mathrm{CH}_{2} \mathrm{Cl}_{2}$ $(12 \mathrm{~mL})$. To the reaction solution was added DMSO $(280 \mu \mathrm{L}, 3.93 \mathrm{mmol})$ and $\mathrm{NEt}_{3}(330 \mu \mathrm{L}, 2.36 \mathrm{mmol})$, and the solution was cooled to $0{ }^{\circ} \mathrm{C}$. Pyridine- $\mathrm{SO}_{3}(250 \mathrm{mg}, 1.57 \mathrm{mmol})$ was added to the reaction in a single portion, and the reaction was allowed to warm to room temperature over 12 hours. The solvent was removed by rotatory evaporation, and the crude residue was dissolved in $20 \mathrm{~mL}$ of EtOAc/ $\mathrm{H}_{2} \mathrm{O}(1: 1)$ and transferred to a separatory funnel. The layers were separated, and the aqueous layer was extracted with additional EtOAc. The combined organic layers were washed with $1 \mathrm{~N} \mathrm{HCl,} \mathrm{sodium}$ bicarbonate, and brine, then dried over magnesium sulfate and concentrated under reduced pressure. The crude product was purified by flash column chromatography ( 0 to $10 \%$ EtOAc in Hexanes) to afford the desired product $\mathbf{1 7}$ as a colorless oil (467 mg, $72 \%$ yield).

${ }^{1} \mathrm{H}$ NMR $(500 \mathrm{MHz}$, Chloroform- $d$ ) $\delta 9.70(\mathrm{~s}, 1 \mathrm{H}), 7.15(\mathrm{~d}, J=8.5 \mathrm{~Hz}, 2 \mathrm{H}), 6.89(\mathrm{~d}, J=2.2 \mathrm{~Hz}, 1 \mathrm{H}), 6.81(\mathrm{~d}, J=8.5 \mathrm{~Hz}$, $2 \mathrm{H}), 6.71(\mathrm{~d}, J=2.1 \mathrm{~Hz}, 1 \mathrm{H}), 6.23(\mathrm{t}, J=2.2 \mathrm{~Hz}, 1 \mathrm{H}), 6.21(\mathrm{~d}, J=2.2 \mathrm{~Hz}, 2 \mathrm{H}), 5.50(\mathrm{~d}, J=6.0 \mathrm{~Hz}, 1 \mathrm{H}), 4.70(\mathrm{~d}, J=6.0$ $\mathrm{Hz}, 1 \mathrm{H}), 1.01$ (s, 9H), 0.98 (s, 9H), 0.93 (s, 18H), 0.25 (s, 6H), 0.19 (s, 6H), 0.12 (s, 6H), 0.12 (s, 6H).

${ }^{13} \mathrm{C}$ NMR (126 MHz, Chloroform- $d$ ) $\delta 190.2,162.2,157.5,157.1,155.9,145.5,133.6,133.2,126.9,124.6,120.4,113.1$, $112.9,111.3,107.7,94.1,55.9,25.83,25.80,18.39,18.36,-4.25,-4.28$.

IR (Neat): 2955, 2929, 2858, 1700, 1608, 1589, 1338, 1254, 1163, 1030, $915 \mathrm{~cm}^{-1}$.

HRMS (ESI) $m / z$ calculated for $\mathrm{C}_{45} \mathrm{H}_{73} \mathrm{O}_{6} \mathrm{Si}_{4}{ }^{+}\left([\mathrm{M}+\mathrm{H}]^{+}\right)$821.4479, found 821.4474 . 
${ }^{1} \mathrm{H}$ NMR, 500 MHz, Chloroform- $d$, Compound 17

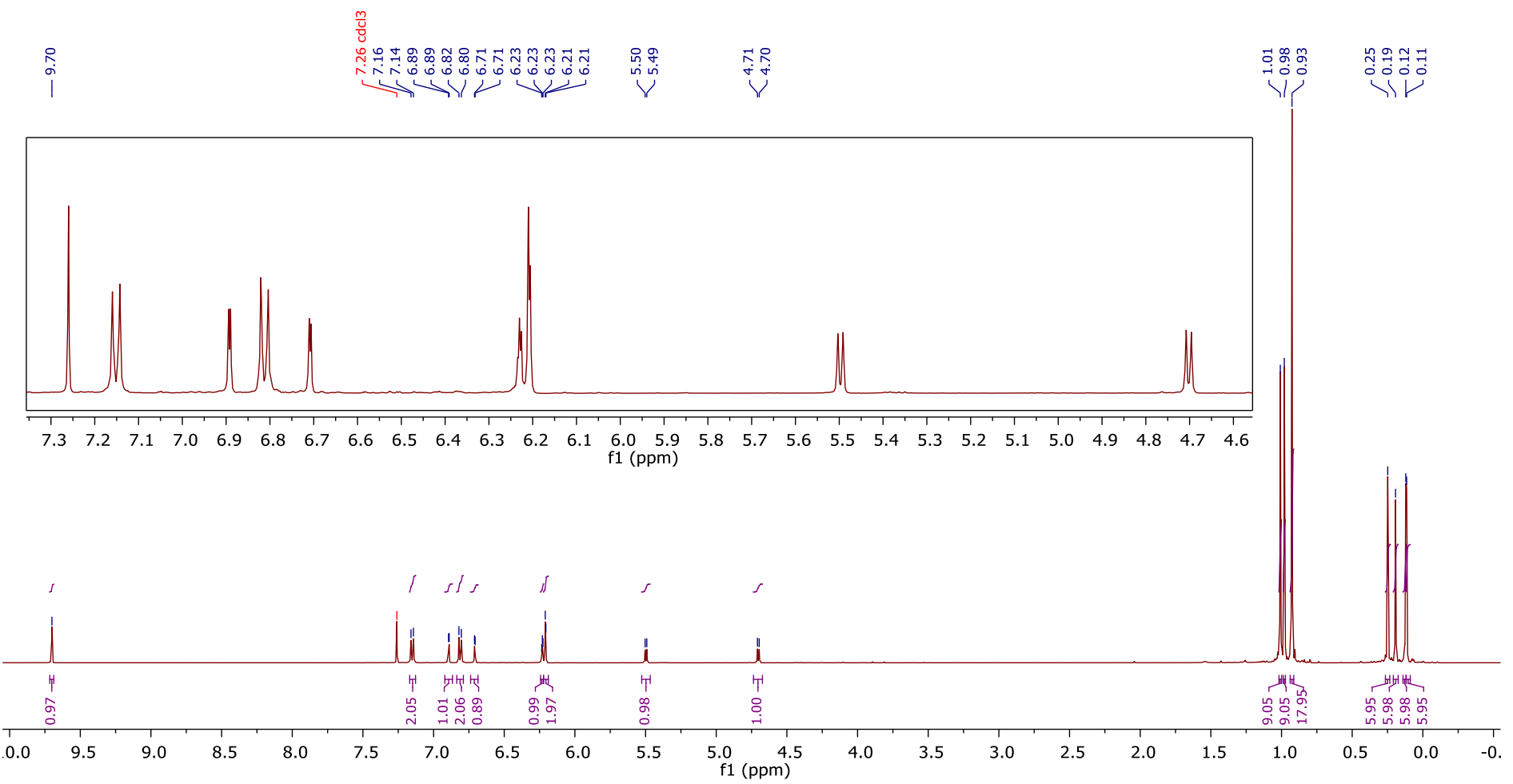

${ }^{13} \mathrm{C}$ NMR, $126 \mathrm{MHz}$, Chloroform- $d$, Compound 17

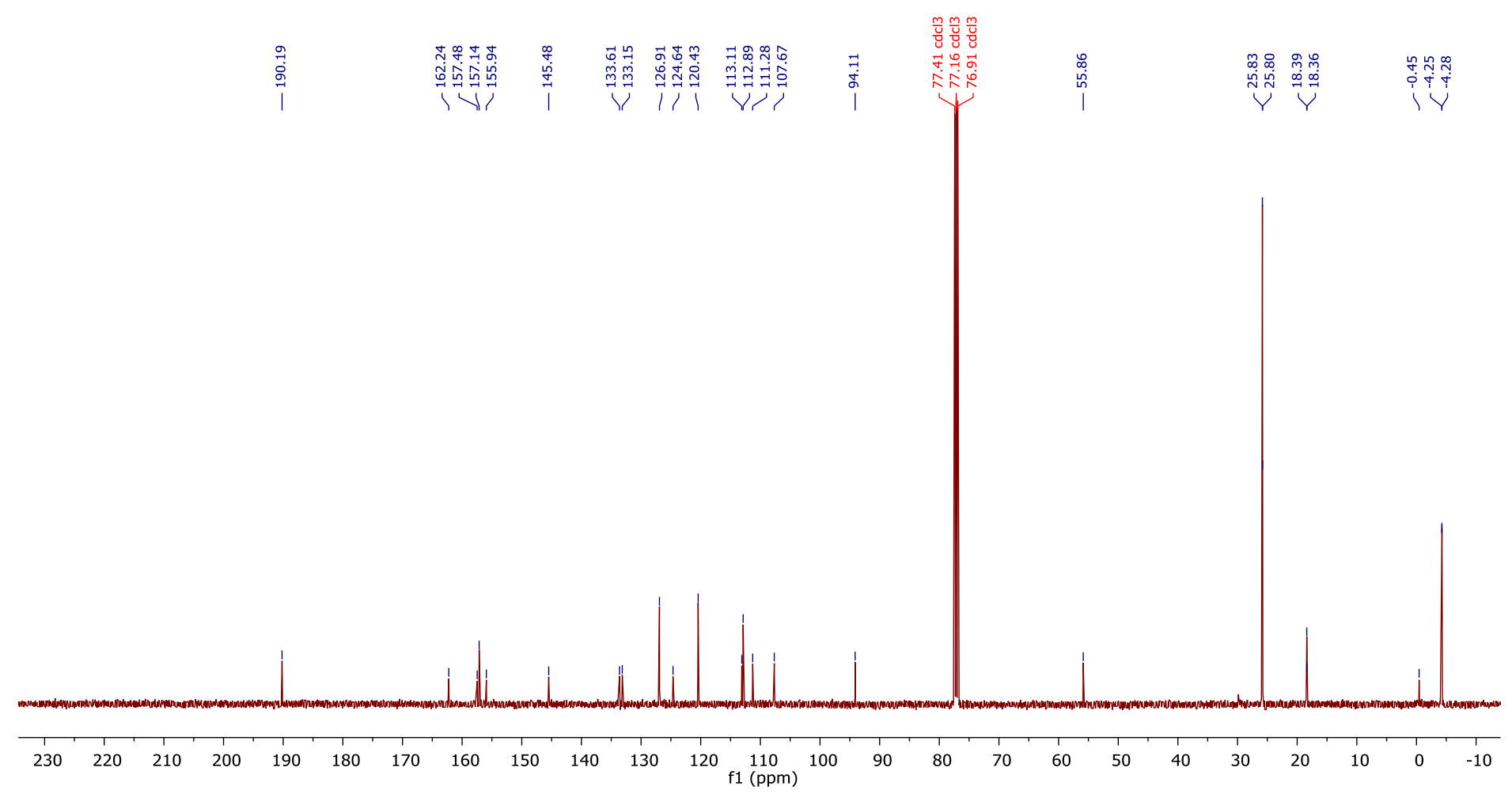



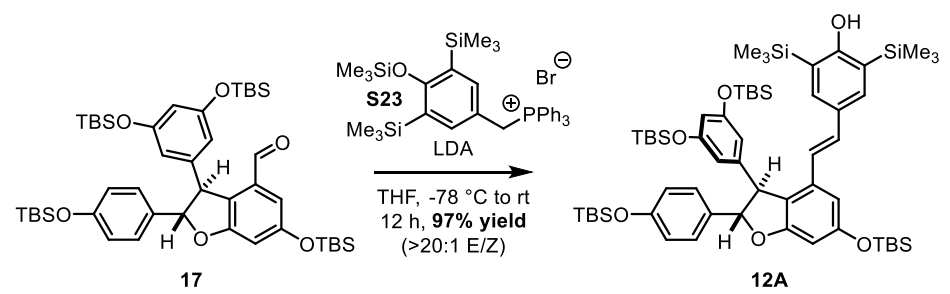

\section{A - 4-((E)-2-((2S,3S)-3-(3,5-bis((tert-butyldimethylsilyl)oxy)phenyl)-6-((tert-butyldimethylsilyl)oxy)-2-(4-((tert- butyldimethylsilyl)oxy)phenyl)-2,3-dihydrobenzofuran-4-yl)vinyl)-2,6-bis(trimethylsilyl)phenol}

\section{Preparation of LDA solution:}

Freshly distilled diisopropyl amine $(126 \mu \mathrm{L}, 0.901 \mathrm{mmol})$ was dissolved in freshly distilled THF $(6 \mathrm{~mL})$ in a flame-dried heart-shaped flask. The resulting solution was cooled to $-78{ }^{\circ} \mathrm{C}$, at which point $n \mathrm{BuLi}(351 \mu \mathrm{L}, 0.877 \mathrm{mmol}, 2.5 \mathrm{M}$ in hexanes) was added. The reaction solution was allowed to stir for $30 \mathrm{~min}$ at $-78{ }^{\circ} \mathrm{C}$ prior to using the LDA solution.

Preparation of the ylide:

Phosphonium salt $\mathbf{S 2 3}$ was prepared as previously reported by our group. ${ }^{4}$ A flame-dried three-neck round bottom flask was charged with $\mathbf{S 2 3}$ (584 mg, $0.877 \mathrm{mmol}$ ) under inert atmosphere, and $\mathbf{S 2 3}$ was suspended in freshly distilled THF (6 mL). The resulting suspension was cooled to $-78{ }^{\circ} \mathrm{C}$, at which point the LDA solution (vide supra) was added to the suspension via cannula (Note: Both solutions were held at $-78{ }^{\circ} \mathrm{C}$ for the duration of the cannulation). As deprotonation to form the ylide occurred, the reaction solution turned a brilliant red color. This solution was allowed to stir at $-78{ }^{\circ} \mathrm{C}$ for 1 hour prior to addition of the aldehyde.

\section{Wittig olefination between the ylide and aldehyde 17:}

Aldehyde 17 (400 mg, $0.487 \mathrm{mmol}$ ) was added to a flame-dried heart-shaped flask and dissolved in freshly distilled THF $(5 \mathrm{~mL})$. Upon completion of the ylide formation, the aldehyde solution was added to the ylide solution via cannula (Note: The aldehyde solution was at room temperature while the ylide solution was held at $-78{ }^{\circ} \mathrm{C}$ for the duration of the cannulation). Upon complete addition of the aldehyde solution, the reaction was allowed to slowly warm to room temperature overnight $(\sim 12 \mathrm{~h})$. The reaction was subsequently diluted with EtOAc $(\sim 25 \mathrm{~mL})$ and added to a separatory funnel containing DI water $(\sim 50 \mathrm{~mL})$. An aqueous solution of saturated $\mathrm{NH}_{4} \mathrm{Cl}(\sim 50 \mathrm{~mL})$ was added to the separatory funnel, and the layers were separated. The aqueous layer was extracted with additional EtOAc, and the combined organic layers were washed with aqueous saturated sodium bicarbonate, washed with brine, dried over $\mathrm{MgSO}_{4}$, and concentrated. The crude product was purified by flash column chromatography ( $5 \%$ to $35 \% \mathrm{CH}_{2} \mathrm{Cl}_{2}$ in $\mathrm{Hexanes} 7$ steps, 2 column volumes per step, $300 \mathrm{~mL}$ column volumes) to afford the desired product as a white foam (498 $\mathrm{mg}, 97 \%$ yield).

${ }^{1} \mathrm{H}$ NMR $(700 \mathrm{MHz}$, Chloroform-d) $\delta 7.17(\mathrm{~d}, J=6.6 \mathrm{~Hz}, 4 \mathrm{H}), 6.85-6.78(\mathrm{~m}, 3 \mathrm{H}), 6.70(\mathrm{~s}, 1 \mathrm{H}), 6.47(\mathrm{~d}, J=16.1 \mathrm{~Hz}, 1 \mathrm{H})$, $6.38(\mathrm{~s}, 1 \mathrm{H}), 6.25-6.20(\mathrm{~m}, 3 \mathrm{H}), 5.34(\mathrm{~d}, J=7.1 \mathrm{~Hz}, 1 \mathrm{H}), 4.99(\mathrm{~s}, 1 \mathrm{H}), 4.42(\mathrm{~d}, J=7.1 \mathrm{~Hz}, 1 \mathrm{H}), 1.03(\mathrm{~s}, 9 \mathrm{H}), 0.98(\mathrm{~s}, 9 \mathrm{H})$, $0.90(\mathrm{~s}, 18 \mathrm{H}), 0.29(\mathrm{~s}, 18 \mathrm{H}), 0.27(\mathrm{~s}, 6 \mathrm{H}), 0.19(\mathrm{~s}, 6 \mathrm{H}), 0.06(\mathrm{~s}, 6 \mathrm{H}), 0.05(\mathrm{~s}, 6 \mathrm{H})$.

${ }^{13} \mathrm{C}$ NMR (176 MHz, Chloroform- $d$ ) $\delta 165.2,161.5,157.1,157.0,155.8,145.0,135.7,135.1,134.1,129.9,129.5,127.2$, $124.3,123.2,120.3,120.0,113.0,111.4,108.9,100.9,93.6,57.0,25.9,25.8,18.4,18.37,18.35,-4.13,-4.15,-4.21,-4.24$, $-4.25,-4.4$.

IR (Neat): 3609, 2944, 2892, 2866, 1607, 1584, 1462, 1340, 1264, 1166, 1012, 960, $852 \mathrm{~cm}^{-1}$.

HRMS (ESI) $m / z$ calculated for $\mathrm{C}_{58} \mathrm{H}_{95} \mathrm{O}_{6} \mathrm{Si}_{6}{ }^{+}\left([\mathrm{M}+\mathrm{H}]^{+}\right) 1055.5739$, found 1055.5725 . 
${ }^{1} \mathrm{H}$ NMR, $700 \mathrm{MHz}$, Chloroform- $d$, Compound 12A
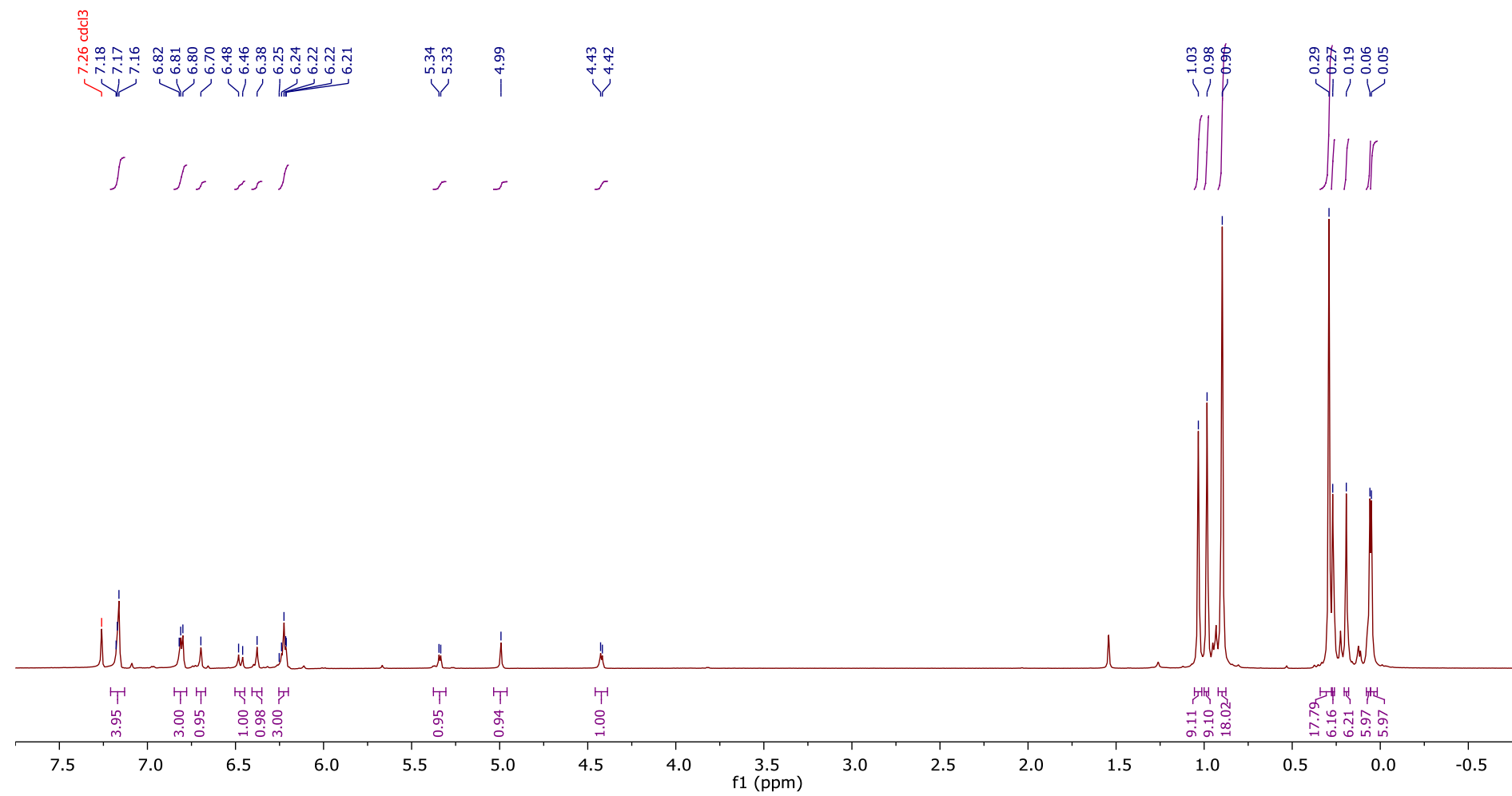

${ }^{13} \mathrm{C}$ NMR, $176 \mathrm{MHz}$, Chloroform- $d$, Compound 12A
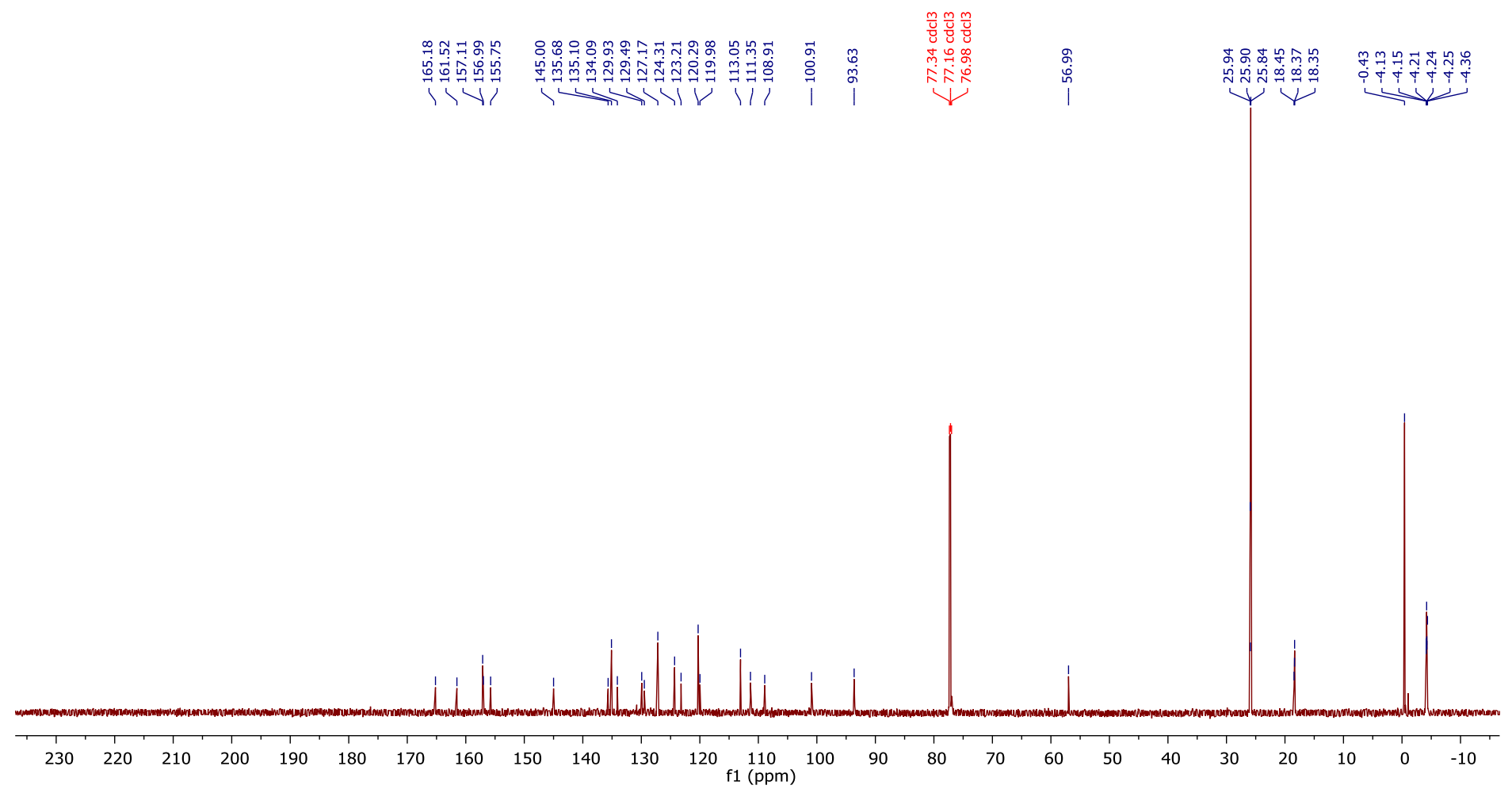


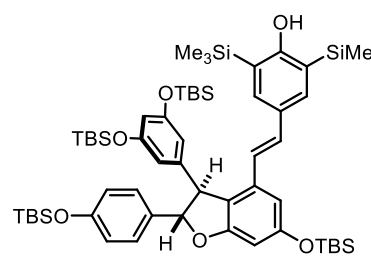

$12 \mathrm{~A}$

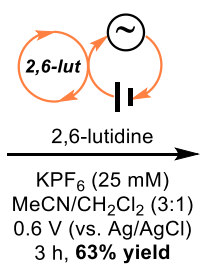

$\mathrm{h}, 63 \%$ yield

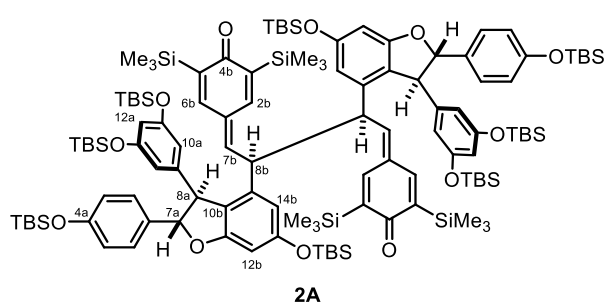

\section{$2 \mathrm{~A}$ - 4,4'-((2S,3S)-2,3-bis((2S,3S)-3-(3,5-bis((tert-butyldimethylsilyl)oxy)phenyl)-6-((tert-butyldimethylsilyl)oxy)-2- (4-((tert-butyldimethylsilyl)oxy)phenyl)-2,3-dihydrobenzofuran-4-yl)butane-1,4-diylidene)bis(2,6- bis(trimethylsilyl)cyclohexa-2,5-dien-1-one)}

Protected $\varepsilon$-viniferin analogue 12A ( $83 \mathrm{mg}, 0.079 \mathrm{mmol}$ ) was added to a $10-\mathrm{mL}$ reaction via charged with a stir bar and $\mathrm{KPF}_{6}\left(36 \mathrm{mg}, 0.2 \mathrm{mmol}, 2.5\right.$ equiv). The solids were dissolved in a 3:1 $\mathrm{MeCN} / \mathrm{CH}_{2} \mathrm{Cl}_{2}(8 \mathrm{~mL})$, and 2,6-lutidine $(2.3 \mu \mathrm{L}$, $0.0197 \mathrm{mmol}, 0.25$ equiv) was added to the reaction solution. Two pieces of $0.25 \mathrm{x} 2$-inch RVC panel ( 0.25 inch thickness) were cut. To each, a hole was made near one end, and copper wire was placed through the hole and wrapped around the top of each electrode. One end of the wire was left free in to connect to the alligator clips. These electrodes were carefully placed into the reaction vial along with the reference electrode $(\mathrm{Ag} / \mathrm{AgCl}$ in $3 \mathrm{M} \mathrm{KCl})$ and a divider (see image). The alligator clips were connected such that the reference (white) and working (green) electrodes were adjacent to each other, while the counter (red) electrode was opposite the divider. Care was taken to ensure the copper wire was not submerged in solvent, nor the active components of the alligator clips touching each other. The reaction was stirred at $750 \mathrm{rpm}$ for $3 \mathrm{~h}$ at a constant voltage of $0.6 \mathrm{~V}$. Upon completion of the reaction, the electrodes were removed and rinsed into a collection flask with DCM $(\sim 40 \mathrm{~mL})$. The contents of the reaction vial were also rinsed into the

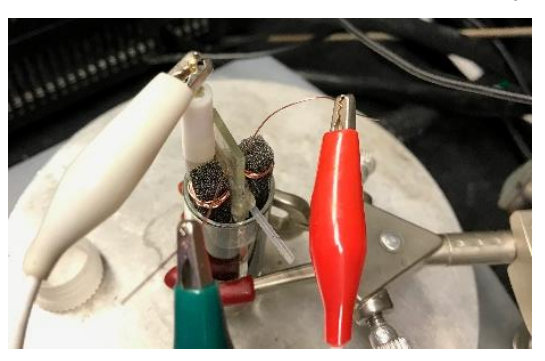
collection flask. The solvent was removed on the rotovap, the crude material was resuspended in DCM, and the electrolyte was filtered away with a plug of Celite. The filtrate was then concentrated and purified by column chromatography (10\% to $75 \%$ DCM in Hexanes) to afford the product as a yellow foam (52 mg, 63\% yield). On $500 \mathrm{mg}$ scale, the reaction was conducted with $1 \times 2$ inch RVC panels ( 0.25 inch thickness) in a $50 \mathrm{~mL}$ beaker in $40 \mathrm{~mL}$ of $\mathrm{MeCN} / \mathrm{CH}_{2} \mathrm{Cl}_{2}$. The same ratio of $\mathrm{KPF}_{6}$ and 2,6-lutidine was used relative to the starting material to yield $312 \mathrm{mg}$ of product. The spectroscopic data for this compound was consistent with our prior report for the benzyl-protected compound. ${ }^{4}$

${ }^{1} \mathrm{H}$ NMR (700 MHz, Chloroform- $d$ ) $\delta 6.94\left(\mathrm{~d}, J=8.5 \mathrm{~Hz}, 4 \mathrm{H}, \mathrm{C}_{2 \mathrm{a}}-\underline{\mathrm{H}}\right), 6.80\left(\mathrm{~d}, J=8.5 \mathrm{~Hz}, 4 \mathrm{H}, \mathrm{C}_{3 \mathrm{a}}-\underline{\mathrm{H}}\right), 6.63(\mathrm{~d}, J=2.6 \mathrm{~Hz}$, $\left.2 \mathrm{H}, \mathrm{C}_{2 / 6 \mathrm{~b}}-\underline{\mathrm{H}}\right), 6.54\left(\mathrm{~d}, J=2.7 \mathrm{~Hz}, 2 \mathrm{H}, \mathrm{C}_{2 / 6 \mathrm{~b}}-\underline{\mathrm{H}}\right), 6.51\left(\mathrm{~d}, J=2.0 \mathrm{~Hz}, 2 \mathrm{H}, \mathrm{C}_{14 \mathrm{~b}}-\underline{\mathrm{H}}\right), 6.25\left(\mathrm{~d}, J=1.9 \mathrm{~Hz}, 2 \mathrm{H}, \mathrm{C}_{12 \mathrm{~b}}-\underline{\mathrm{H}}\right), 5.99(\mathrm{t}$, $\left.J=2.2 \mathrm{~Hz}, 2 \mathrm{H}, \mathrm{C}_{12 \mathrm{a}}-\underline{\mathrm{H}}\right), 5.98-5.95\left(\mathrm{~m}, 2 \mathrm{H}, \mathrm{C}_{7 \mathrm{~b}}-\underline{\mathrm{H}}\right), 4.99\left(\mathrm{~d}, J=7.6 \mathrm{~Hz}, 2 \mathrm{H}, \mathrm{C}_{7 \mathrm{a}}-\underline{\mathrm{H}}\right), 4.01\left(\mathrm{~d}, J=7.6 \mathrm{~Hz}, 2 \mathrm{H}, \mathrm{C}_{8 \mathrm{a}}-\underline{\mathrm{H}}\right), 3.99$ $\left(\mathrm{dd}, J=7.4,2.9 \mathrm{~Hz}, 2 \mathrm{H}, \mathrm{C}_{8 \mathrm{~b}}-\underline{\mathrm{H}}\right), 1.01(\mathrm{~s}, 18 \mathrm{H}), 0.97(\mathrm{~s}, 18 \mathrm{H}), 0.86(\mathrm{~s}, 36 \mathrm{H}), 0.22(\mathrm{~s}, 6 \mathrm{H}), 0.21(\mathrm{~s}, 12 \mathrm{H}), 0.18(\mathrm{~s}, 6 \mathrm{H}), 0.16$ $(\mathrm{s}, 18 \mathrm{H}), 0.03(\mathrm{~s}, 18 \mathrm{H}),-0.06(\mathrm{~s}, 24 \mathrm{H})$.

${ }^{13} \mathrm{C}$ NMR (176 MHz, Chloroform- $d$ ) $\delta$ 192.7, 161.3, 157.3, 156.0, 148.3, 144.5, 142.0, 141.3, 139.8, 138.2, 133.1, 132.5, $127.6,121.7,120.3,111.1,101.1,94.2,55.9,49.4,25.9,25.8,25.8,18.4,18.31,18.28,-1.2,-1.3,-4.1,-4.17,-4.24$.

HRMS (ESI) $m / z$ calculated for $\mathrm{C}_{116} \mathrm{H}_{187} \mathrm{O}_{12} \mathrm{Si}_{12}{ }^{+}\left([\mathrm{M}+\mathrm{H}]^{+}\right)$2108.1248, found 2108.1228.

Note: The $\mathrm{C}_{10 \mathrm{a}}-\mathrm{H}$ protons are not visible due to extreme broadening in the ${ }^{1} \mathrm{H}$ NMR spectrum. This is presumably due to hindered/slow rotation in a sterically encumbered environment. In the ${ }^{13} \mathrm{C} N M R$ spectrum, the resonances for $\mathrm{C}_{9 \mathrm{a}}-\mathrm{C}_{12 \mathrm{a}}$ were very broadened, further corroborating that rotation of the bulky phenol is likely hindered in this sterically congested system. 
${ }^{1} \mathrm{H}$ NMR, $700 \mathrm{MHz}$, Chloroform- $d$, Compound 2A

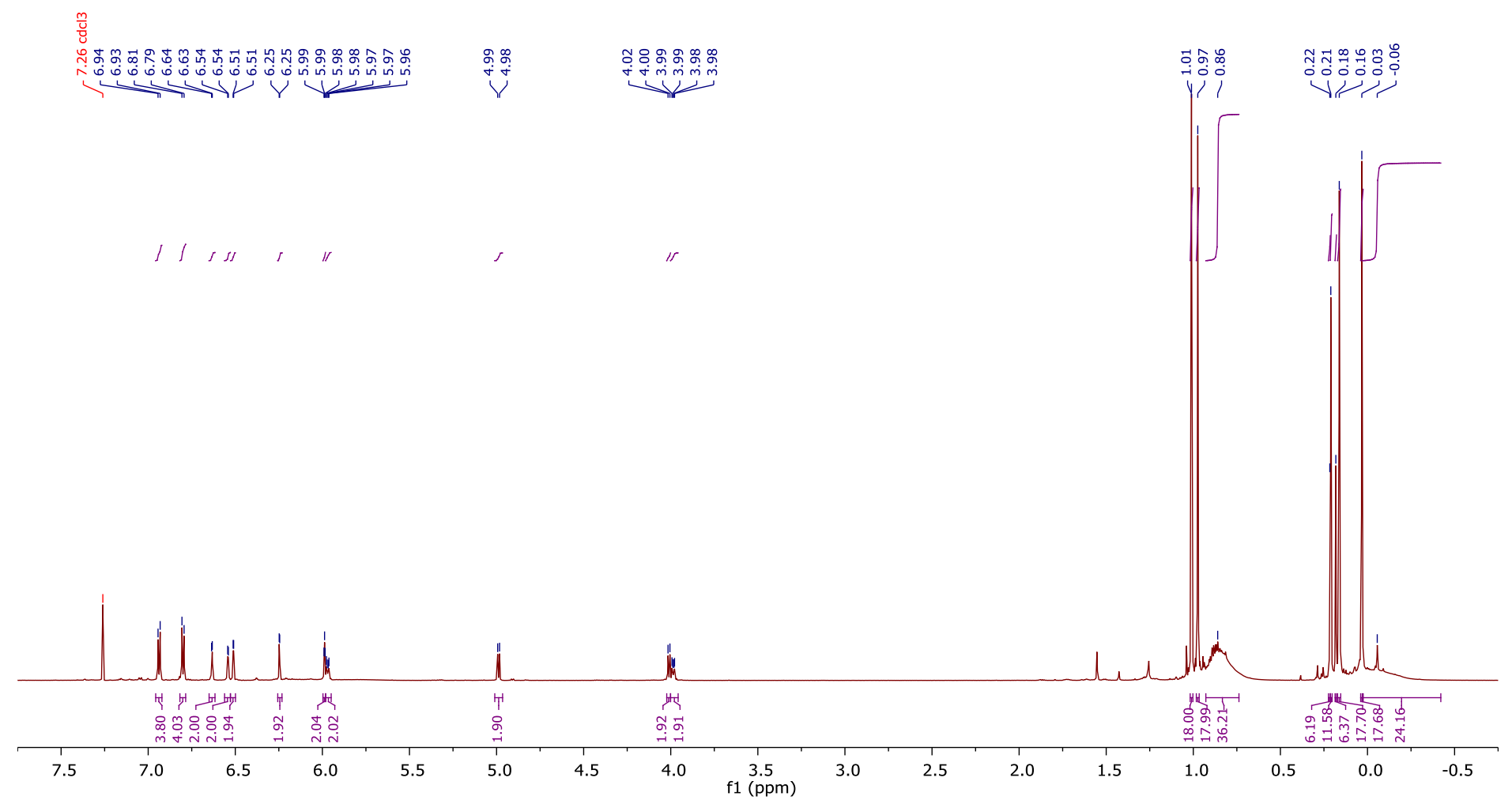

${ }^{13} \mathrm{C}$ NMR, $176 \mathrm{MHz}$, Chloroform- $d$, Compound 2A

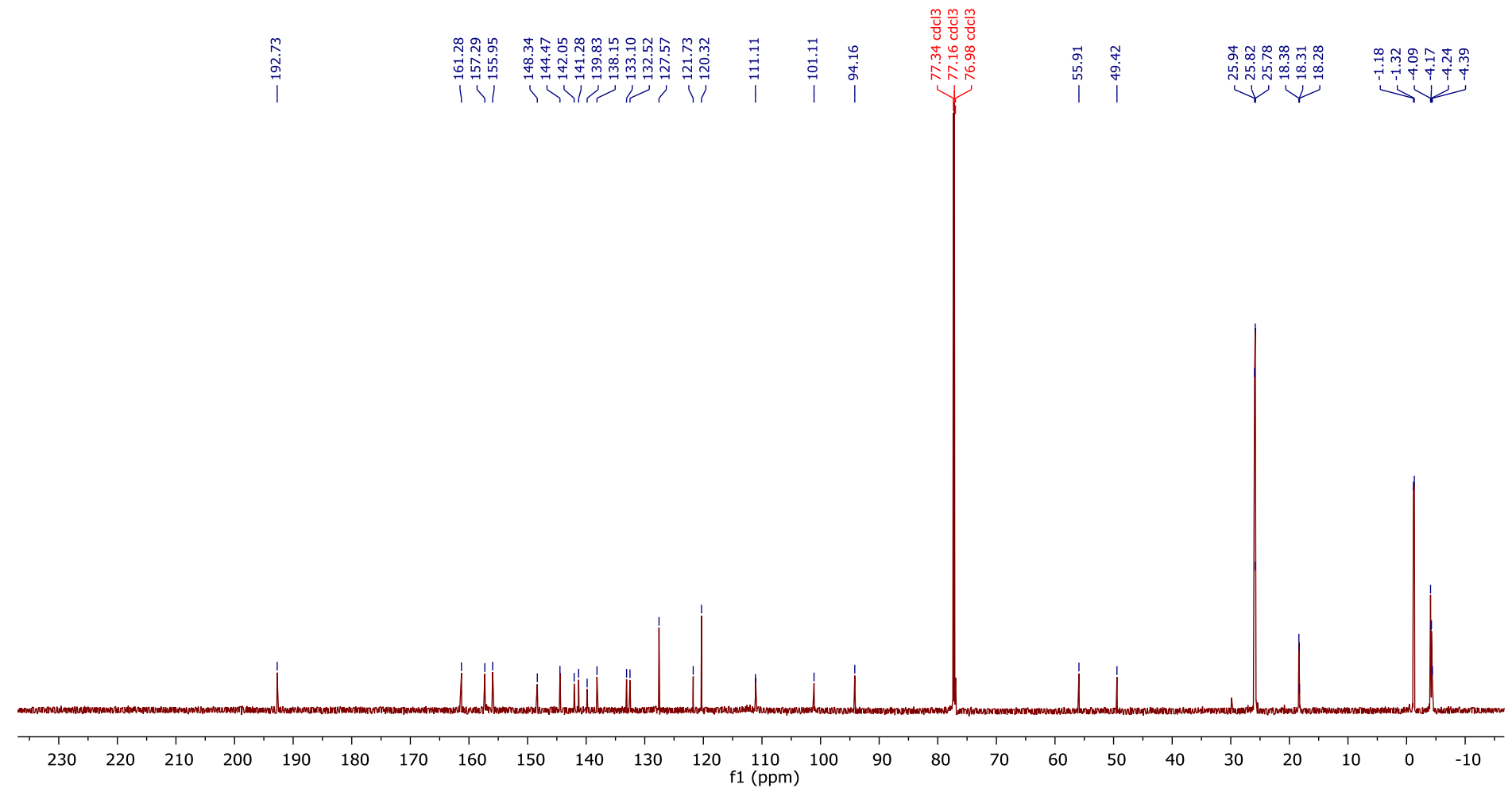




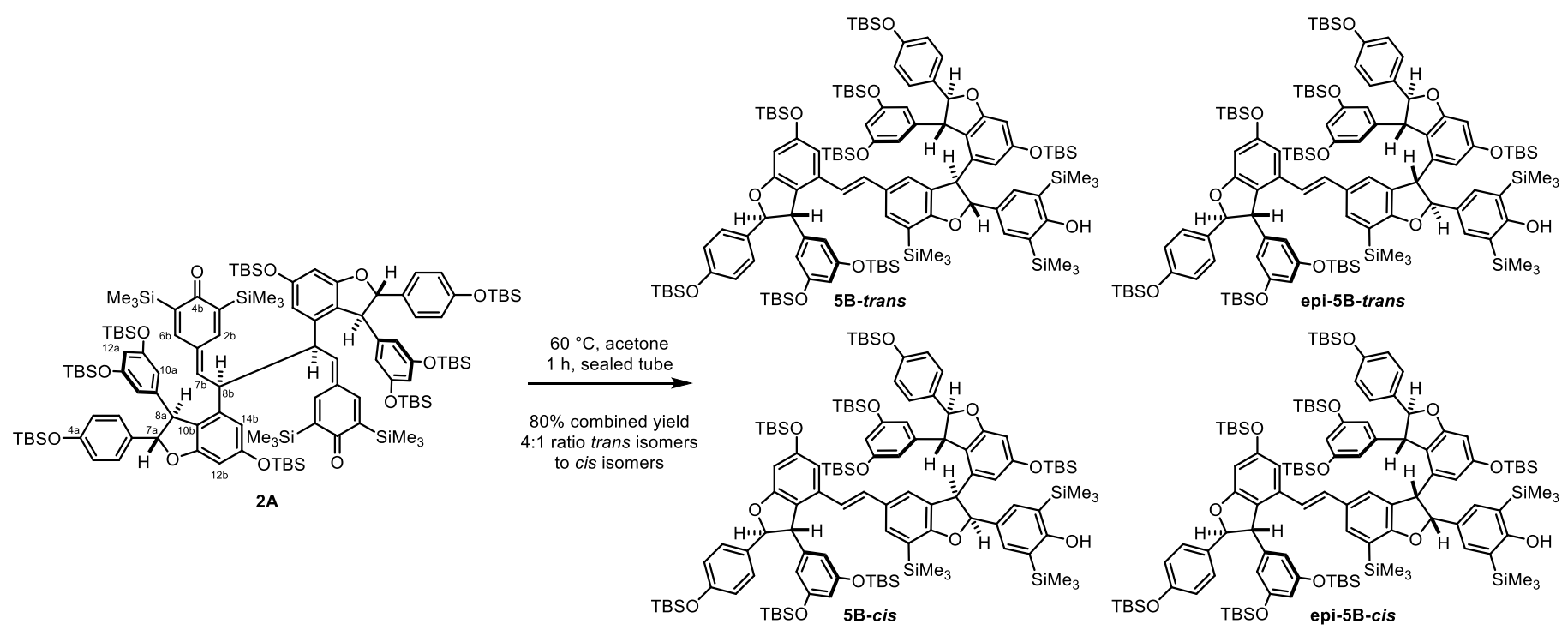

5B - 4-((2R,2'S,3R,3'S)-3'-(3,5-bis((tert-butyldimethylsilyl)oxy)phenyl)-5-((E)-2-((2S,3S)-3-(3,5-bis((tertbutyldimethylsilyl)oxy)phenyl)-6-((tert-butyldimethylsilyl)oxy)-2-(4-((tert-butyldimethylsilyl)oxy)phenyl)-2,3dihydrobenzofuran-4-yl)vinyl)-6' -((tert-butyldimethylsilyl)oxy)-2'-(4-((tert-butyldimethylsilyl)oxy)phenyl)-7(trimethylsilyl)-2,2',3,3'-tetrahydro-[3,4'-bibenzofuran]-2-yl)-2,6-bis(trimethylsilyl)phenol

Quinone-methide dimer $2 \mathrm{~A}$ (50 $\mathrm{mg}, 0.024 \mathrm{mmol}$ ) was added to a flame-dried reaction vial charged with a stir bar and dissolved in acetone (1.0 mL, HPLC grade). The reaction solution was sparged with a balloon of Ar for 5 minutes and then sealed under argon with parafilm. The reaction was heated to $60{ }^{\circ} \mathrm{C}$ for 1 hour, at which point it was cooled to room temperature, the stir bar was removed, and the solvent was evaporated under reduced pressure. The crude reaction was purified by flash column chromatography (4\% to $28 \%$ over 7 steps, then $33 \%, 50 \%, 66 \% \mathrm{CH}_{2} \mathrm{Cl}_{2}$ in $\mathrm{Hexanes}_{1} 1$ column volume per step, $30 \mathrm{~mL}$ column volume) to afford the inseparable mixture of four diastereomers (38.6 $\mathrm{mg}, 80 \%$ yield). Additional rounds of chromatography afforded modest resolution between the trans-DHB and cis-DHB isomers; however, 5B-trans and epi-5B-trans were not separable from each other, so for targeting the natural products the crude mixture was carried forward for desilylation without purification (see below).

\section{B-trans/epi-5B-trans mixture:}

${ }^{1} \mathrm{H}$ NMR $(700 \mathrm{MHz}$, Chloroform- $d$ ) $\delta 7.15(\mathrm{t}, J=8.7 \mathrm{~Hz}, 5 \mathrm{H}), 7.11(\mathrm{~d}, J=2.0 \mathrm{~Hz}, 1 \mathrm{H}), 7.10(\mathrm{~d}, J=2.9 \mathrm{~Hz}, 2 \mathrm{H}), 7.09$ (s, $1 \mathrm{H}), 6.96(\mathrm{~s}, 1 \mathrm{H}), 6.94(\mathrm{~s}, 1 \mathrm{H}), 6.90(\mathrm{~d}, J=3.0 \mathrm{~Hz}, 4 \mathrm{H}), 6.80(\mathrm{~d}, J=8.5 \mathrm{~Hz}, 2 \mathrm{H}), 6.78(\mathrm{~d}, J=2.3 \mathrm{~Hz}, 2 \mathrm{H}), 6.76(\mathrm{~d}, J=8.4$ $\mathrm{Hz}, 4 \mathrm{H}), 6.62(\mathrm{dd}, J=17.3,2.4 \mathrm{~Hz}, 5 \mathrm{H}), 6.57(\mathrm{~d}, J=16.2 \mathrm{~Hz}, 1 \mathrm{H}), 6.39(\mathrm{~d}, J=2.1 \mathrm{~Hz}, 2 \mathrm{H}), 6.37(\mathrm{~d}, J=16.1 \mathrm{~Hz}, 1 \mathrm{H}), 6.34$ $(\mathrm{d}, J=2.1 \mathrm{~Hz}, 2 \mathrm{H}), 6.31(\mathrm{~d}, J=16.2 \mathrm{~Hz}, 1 \mathrm{H}), 6.21(\mathrm{~d}, J=2.2 \mathrm{~Hz}, 2 \mathrm{H}), 6.20(\mathrm{~s}, 3 \mathrm{H}), 6.17(\mathrm{~s}, 3 \mathrm{H}), 5.99(\mathrm{t}, J=2.2 \mathrm{~Hz}, 1 \mathrm{H})$, $5.96(\mathrm{t}, J=2.0 \mathrm{~Hz}, 1 \mathrm{H}), 5.92(\mathrm{~s}, 4 \mathrm{H}), 5.36(\mathrm{~d}, J=5.2 \mathrm{~Hz}, 1 \mathrm{H}), 5.35(\mathrm{~d}, J=5.3 \mathrm{~Hz}, 1 \mathrm{H}), 5.32(\mathrm{~d}, J=6.7 \mathrm{~Hz}, 1 \mathrm{H}), 5.31(\mathrm{~d}, J$ $=6.8 \mathrm{~Hz}, 1 \mathrm{H}), 5.23(\mathrm{dd}, J=4.3,1.7 \mathrm{~Hz}, 2 \mathrm{H}), 4.84(\mathrm{~s}, 1 \mathrm{H}), 4.82(\mathrm{~s}, 1 \mathrm{H}), 4.39(\mathrm{~d}, J=6.6 \mathrm{~Hz}, 1 \mathrm{H}), 4.37(\mathrm{~d}, J=6.5 \mathrm{~Hz}, 1 \mathrm{H})$, $4.29(\mathrm{t}, J=4.7 \mathrm{~Hz}, 2 \mathrm{H}), 4.13(\mathrm{~d}, J=5.1 \mathrm{~Hz}, 1 \mathrm{H}), 4.12(\mathrm{~d}, J=5.0 \mathrm{~Hz}, 1 \mathrm{H}), 1.03(\mathrm{~s}, 9 \mathrm{H}), 1.01(\mathrm{~s}, 9 \mathrm{H}), 0.98(\mathrm{~s}, 9 \mathrm{H}), 0.97(\mathrm{~s}$, 9H), $0.96(\mathrm{~s}, 9 \mathrm{H}), 0.95(\mathrm{~s}, 9 \mathrm{H}), 0.94(\mathrm{~s}, 9 \mathrm{H}), 0.94(\mathrm{~s}, 9 \mathrm{H}), 0.88(\mathrm{~s}, 18 \mathrm{H}), 0.87(\mathrm{~d}, J=1.1 \mathrm{~Hz}, 36 \mathrm{H}), 0.87(\mathrm{~s}, 18 \mathrm{H}), 0.26(\mathrm{~s}$, $6 \mathrm{H}), 0.24(\mathrm{~s}, 3 \mathrm{H}), 0.24(\mathrm{~s}, 3 \mathrm{H}), 0.21(\mathrm{~s}, 18 \mathrm{H}), 0.20(\mathrm{~s}, 18 \mathrm{H}), 0.19(\mathrm{~s}, 9 \mathrm{H}), 0.18(\mathrm{~d}, J=1.2 \mathrm{~Hz}, 17 \mathrm{H}), 0.17(\mathrm{~s}, 9 \mathrm{H}), 0.16(\mathrm{~s}$, $6 \mathrm{H}), 0.15(\mathrm{~d}, J=1.5 \mathrm{~Hz}, 12 \mathrm{H}), 0.14(\mathrm{~s}, 6 \mathrm{H}), 0.13(\mathrm{~s}, 3 \mathrm{H}), 0.12(\mathrm{~d}, J=1.6 \mathrm{~Hz}, 3 \mathrm{H})$.

HRMS (ESI) $m / z$ calculated for $\mathrm{C}_{113} \mathrm{H}_{179} \mathrm{O}_{12} \mathrm{Si}_{11}{ }^{+}\left([\mathrm{M}+\mathrm{H}]^{+}\right)$2036.0853, found 2036.0849. 
${ }^{1} \mathrm{H}$ NMR, $500 \mathrm{MHz}$, Chloroform-d, 5B-trans/5B-cis/epi-5B-trans/epi-5B-cis mixture

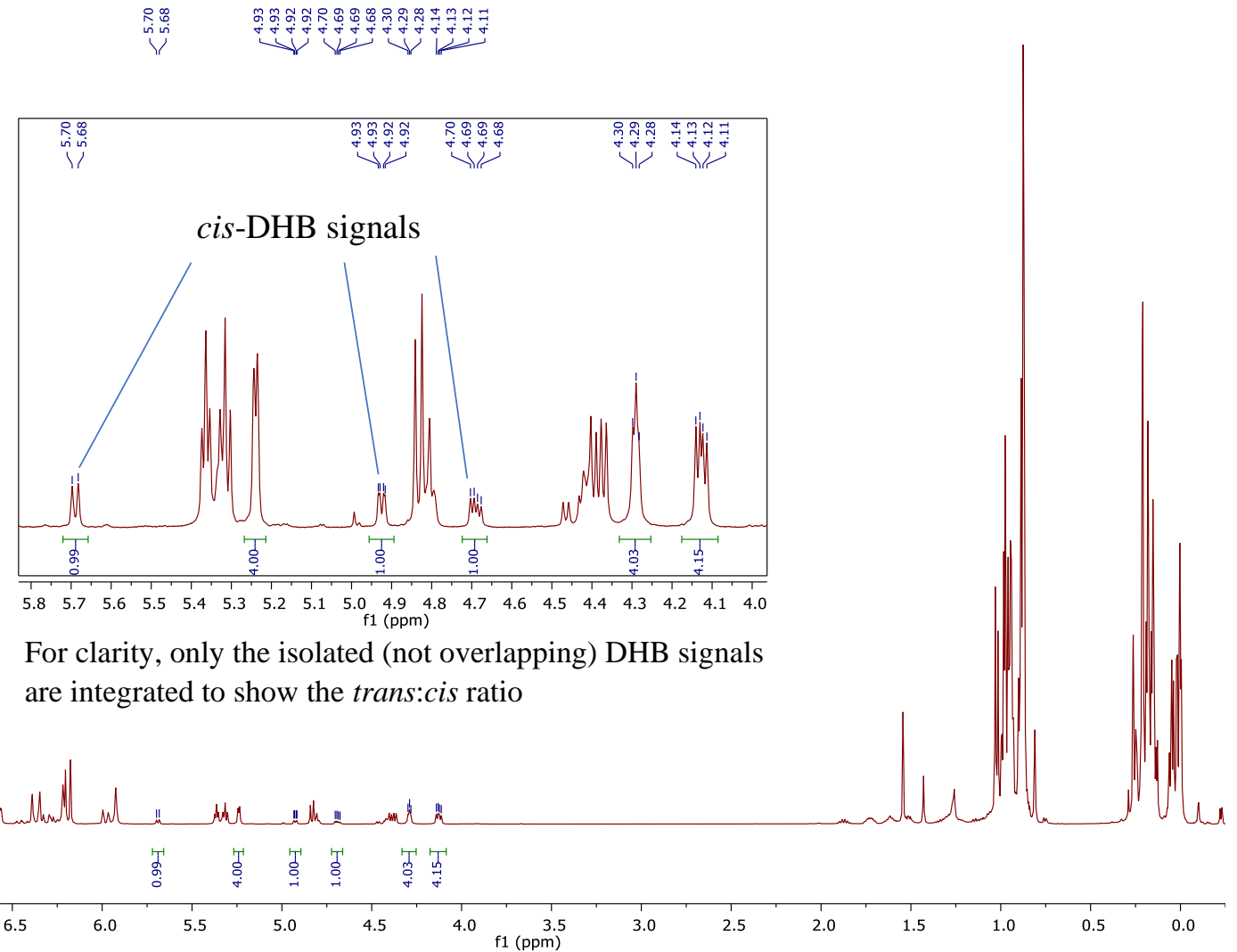

${ }^{1} \mathrm{H}$ NMR, $700 \mathrm{MHz}$, Chloroform-d, 5B-trans/epi-5B-trans mixture

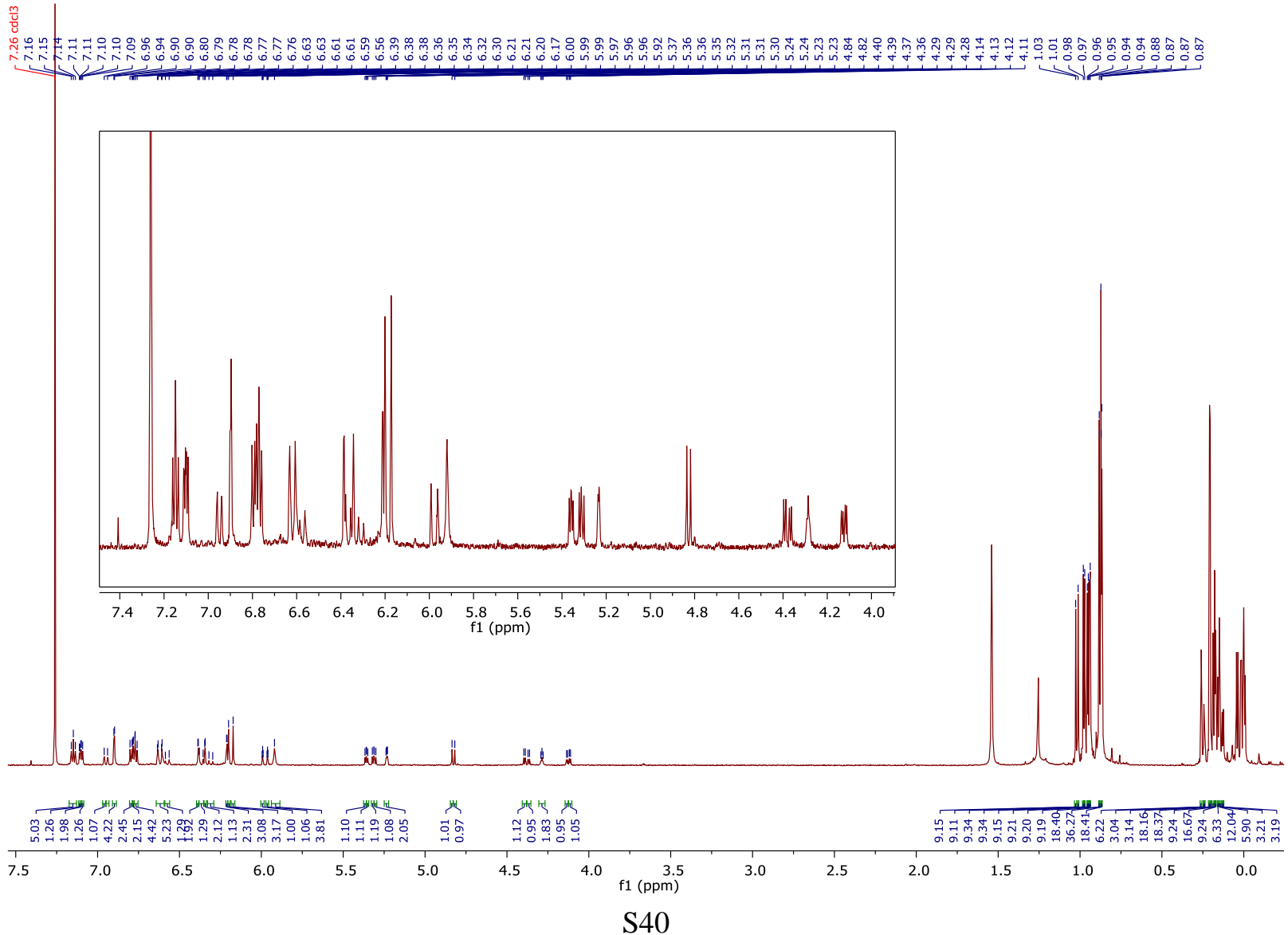



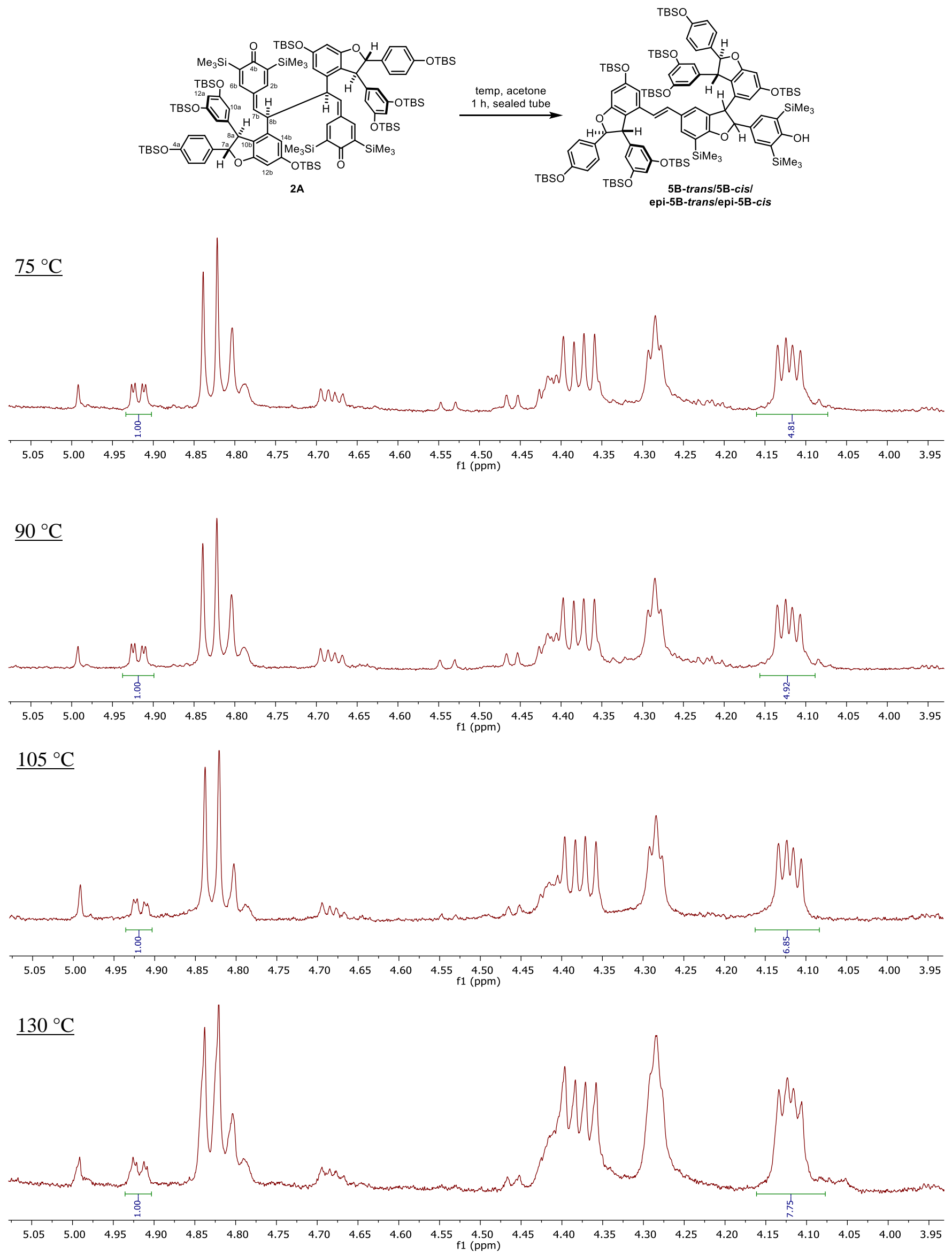

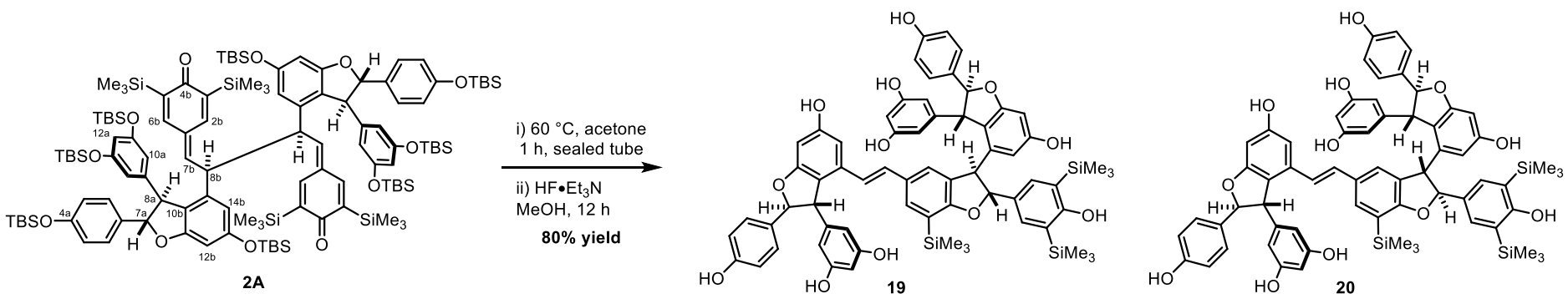

19 - 5-((2S,3S)-4-((E)-2-((2R,2'S,3R,3'S)-3'-(3,5-dihydroxyphenyl)-6'-hydroxy-2-(4-hydroxy-3,5bis(trimethylsilyl)phenyl)-2'-(4-hydroxyphenyl)-7-(trimethylsilyl)-2,2',3,3'-tetrahydro-[3,4'-bibenzofuran]-5yl)vinyl)-6-hydroxy-2-(4-hydroxyphenyl)-2,3-dihydrobenzofuran-3-yl)benzene-1,3-diol

Quinone-methide dimer 2A (100 mg, $0.047 \mathrm{mmol}$ ) was added to a flame-dried reaction vial charged with a stir bar and dissolved in acetone (1.5 mL, HPLC grade). The reaction solution was sparged with a balloon of Ar for 5 minutes and then sealed under argon with parafilm. The reaction was heated to $60{ }^{\circ} \mathrm{C}$ for 1 hour, at which point it was cooled to room temperature, and $\mathrm{HF}_{-} \mathrm{Et}_{3} \mathrm{~N}(0.48 \mathrm{~mL}, 2.94 \mathrm{mmol})$ was added followed by methanol $(1.5 \mathrm{~mL})$. The desilylation stirred for 12 hours, at which point it was transferred to a separatory funnel containing aqueous sat. sodium bicarbonate $(\sim 50 \mathrm{~mL})$, and the aqueous layer was extracted with portions EtOAc $(3 \times 20 \mathrm{~mL})$. The combined organic layers were washed with brine, dried over $\mathrm{MgSO}_{4}$, and concentrated. For characterization purposes this material was purified by flash column chromatography ( $10 \%$ to $40 \%$ in 4 step increments, 2 column volumes per step, Nonpolar solvent $-\mathrm{CH}_{2} \mathrm{Cl}_{2}$, Polar solvent - 3:1 Acetone/MeOH) to afford the 19/20 mixture (44 mg, 80\% yield). Otherwise when targeting vitisin D (7) and compounds 26, 27, and 28, this material was carried forward without purification.

${ }^{1} \mathrm{H}$ NMR $\left(700 \mathrm{MHz}\right.$, Acetone- $\left.d_{6}\right) \delta 8.28(\mathrm{~s}, 15 \mathrm{H}), 7.26-7.18(\mathrm{~m}, 10 \mathrm{H}), 7.16(\mathrm{~d}, J=7.4 \mathrm{~Hz}, 2 \mathrm{H}), 7.06(\mathrm{~d}, J=7.5 \mathrm{~Hz}, 4 \mathrm{H})$, $6.89(\mathrm{dd}, J=12.3,8.1 \mathrm{~Hz}, 4 \mathrm{H}), 6.86-6.81(\mathrm{~m}, 7 \mathrm{H}), 6.77-6.68(\mathrm{~m}, 5 \mathrm{H}), 6.60(\mathrm{~d}, J=6.4 \mathrm{~Hz}, 1 \mathrm{H}), 6.58(\mathrm{~d}, J=6.4 \mathrm{~Hz}, 1 \mathrm{H})$, $6.35(\mathrm{~d}, J=2.1 \mathrm{~Hz}, 1 \mathrm{H}), 6.31(\mathrm{ddd}, J=6.7,4.3,2.0 \mathrm{~Hz}, 4 \mathrm{H}), 6.28(\mathrm{~d}, J=2.0 \mathrm{~Hz}, 1 \mathrm{H}), 6.23(\mathrm{p}, J=1.8 \mathrm{~Hz}, 2 \mathrm{H}), 6.18(\mathrm{~d}, J=$ $2.5 \mathrm{~Hz}, 3 \mathrm{H}), 6.17-6.15(\mathrm{~m}, 2 \mathrm{H}), 6.01-5.98(\mathrm{~m}, 2 \mathrm{H}), 5.94(\mathrm{~d}, J=2.0 \mathrm{~Hz}, 2 \mathrm{H}), 5.92(\mathrm{~d}, J=2.1 \mathrm{~Hz}, 1 \mathrm{H}), 5.60(\mathrm{~d}, J=6.1$ $\mathrm{Hz}, 1 \mathrm{H}), 5.59(\mathrm{~d}, J=5.4 \mathrm{~Hz}, 1 \mathrm{H}), 5.36(\mathrm{t}, J=5.8 \mathrm{~Hz}, 2 \mathrm{H}), 5.31(\mathrm{t}, J=3.7 \mathrm{~Hz}, 2 \mathrm{H}), 4.49(\mathrm{~d}, J=4.0 \mathrm{~Hz}, 1 \mathrm{H}), 4.48-4.42(\mathrm{~m}$, $3 \mathrm{H}), 4.27(\mathrm{dd}, J=8.9,5.7 \mathrm{~Hz}, 2 \mathrm{H}), 0.23(\mathrm{~d}, J=1.1 \mathrm{~Hz}, 18 \mathrm{H}), 0.21(\mathrm{dd}, J=2.9,1.0 \mathrm{~Hz}, 27 \mathrm{H}), 0.18(\mathrm{~s}, 9 \mathrm{H})$.

${ }^{13} \mathrm{C}$ NMR (176 MHz, Acetone- $\left.d_{6}\right) \delta 166.4,164.8,162.8,162.7,160.2,159.91,159.87,159.61,159.57,158.3,158.12$, 158.07, 147.4, 147.3, 146.9, 146.8, 136.72, 136.67, 134.65, 134.60, 134.40, 134.36, 134.1, 133.9, 133.8, 132.2, 132.1, 131.6, $131.0,130.9,130.8,128.32,128.25,128.15,127.9,127.8,126.8,126.7,126.2,123.5,123.4,120.6,119.65,119.62,119.5$, 119.3, 116.5, 116.3, 116.2, 107.01, 106.98, 106.45, 106.42, 104.5, 102.4, 102.3, 96.8, 96.7, 94.25, 94.21, 94.18, 94.16, 91.4, $91.3,69.8,57.3,57.1,57.0,56.7,53.0,-0.3,-0.85,-0.86$.

IR (Neat): 3438 (br), 2925, 1613, 1589, 1524, 1442, 1336, 1269, 1154, 1135, $1012 \mathrm{~cm}^{-1}$.

HRMS (ESI) $m / z$ calculated for $\mathrm{C}_{65} \mathrm{H}_{67} \mathrm{O}_{12} \mathrm{Si}_{3}{ }^{+}\left([\mathrm{M}+\mathrm{H}]^{+}\right)$1123.3935, found 1123.3924. 
${ }^{1} \mathrm{H}$ NMR, $700 \mathrm{MHz}$, Acetone- $d_{6}$, Compounds 19/20

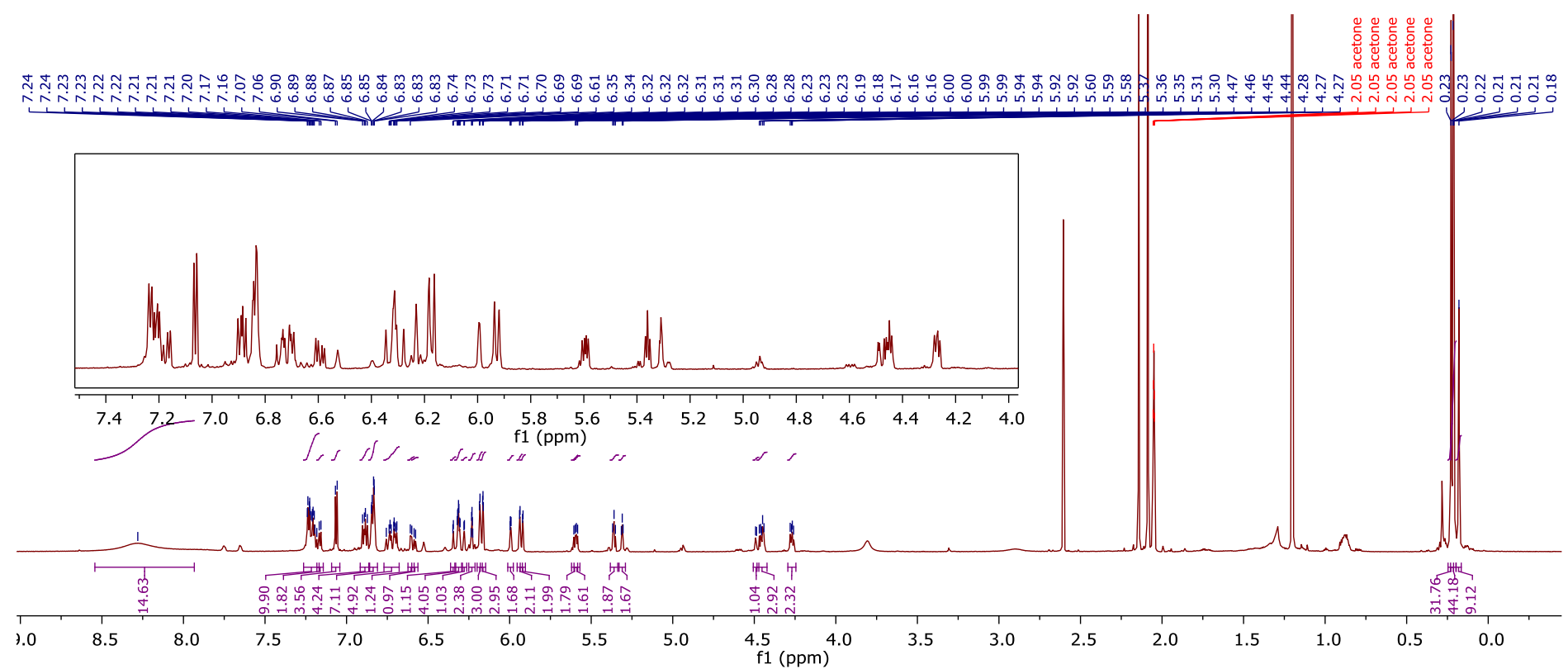

${ }^{13} \mathrm{C}$ NMR, $176 \mathrm{MHz}$, Acetone- $d_{6}$, Compounds 19/20

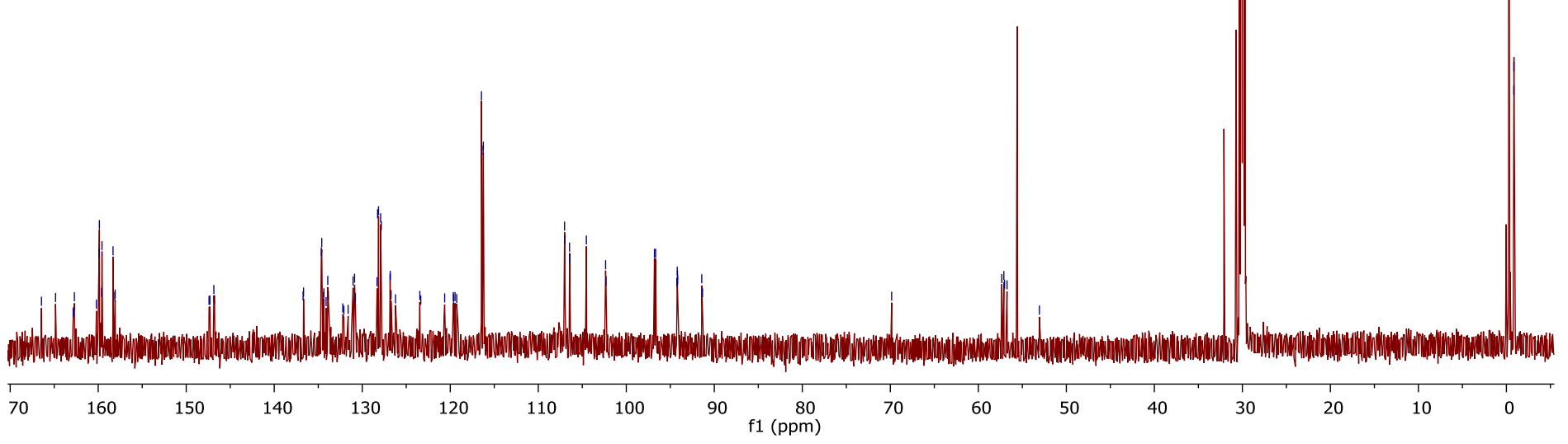



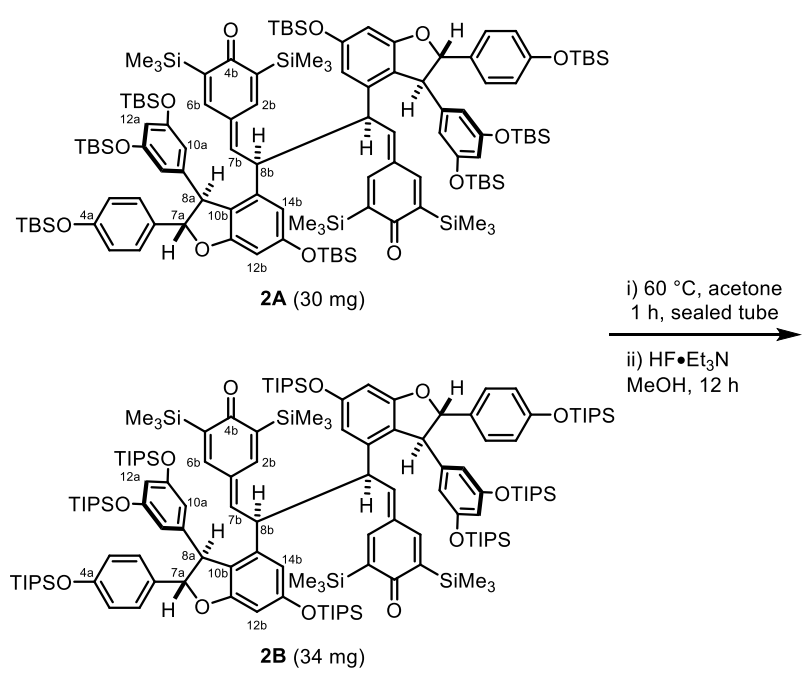
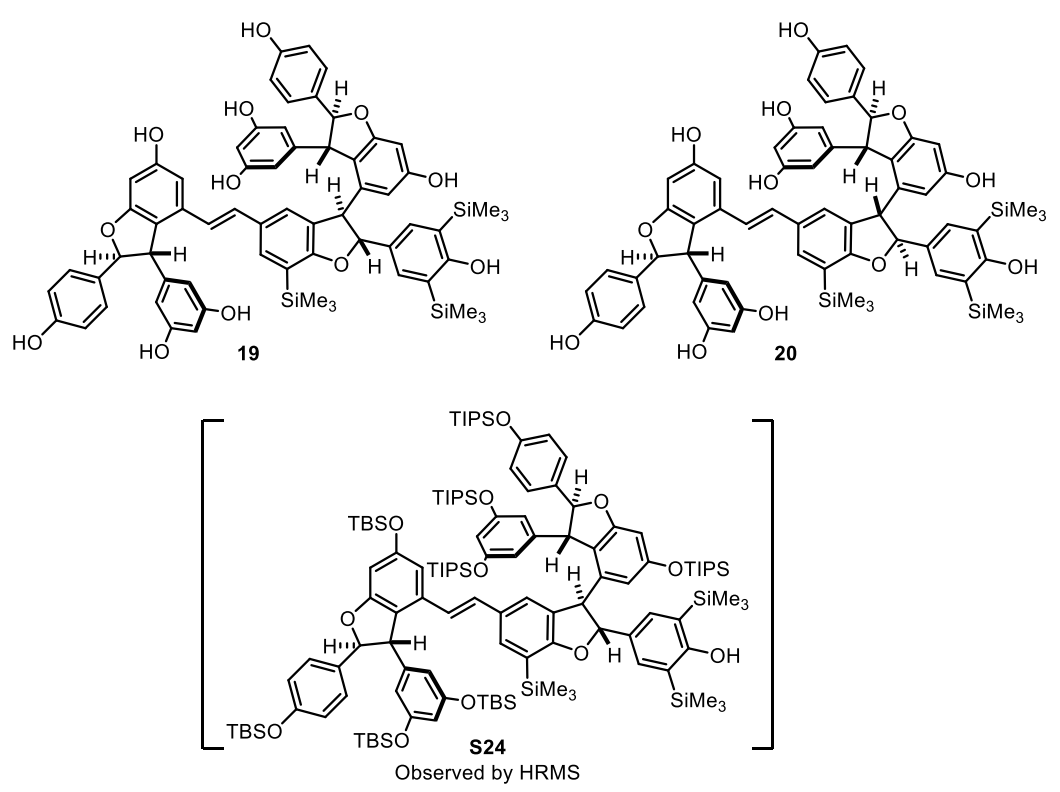

\section{Crossover Experiment with 2A and 2B}

Quinone-methide dimer 2A (30 mg, $0.014 \mathrm{mmol})$ and QMD 2B (34 mg, $0.014 \mathrm{mmol})$ were added to a flame-dried reaction vial charged with a stir bar and dissolved in acetone $(1.0 \mathrm{~mL}$, HPLC grade). The reaction solution was sparged with a balloon of Ar for 5 minutes and then sealed under argon with parafilm. The reaction was heated to $60{ }^{\circ} \mathrm{C}$ for 1 hour, at which point it was cooled to room temperature, and a $50 \mu \mathrm{L}$ sample for HRMS analysis was taken. To the remaining $\mathrm{HF}-\mathrm{Et}_{3} \mathrm{~N}(0.28 \mathrm{~mL}$, $1.71 \mathrm{mmol})$ was added followed by methanol $(1.0 \mathrm{~mL})$. The desilylation stirred for 12 hours, at which point it was transferred to a separatory funnel containing aqueous sat. sodium bicarbonate $(\sim 50 \mathrm{~mL})$, and the aqueous layer was extracted with portions EtOAc $(3 \times 20 \mathrm{~mL})$. The combined organic layers were washed with brine, dried over $\mathrm{MgSO}_{4}$, and concentrated. The crude product was purified by flash column chromatography (10\% to $40 \%$ in 4 step increments, 2 column volumes per step, Nonpolar solvent $-\mathrm{CH}_{2} \mathrm{Cl}_{2}$, Polar solvent $-3: 1$ Acetone/MeOH) to afford the $\mathbf{1 9 / 2 0}$ mixture (12 mg, $75 \%$ yield). See above for characterization of 19/20.

\section{Intermediate S24:}

HRMS (ESI) $m / z$ calculated for $\mathrm{C}_{125} \mathrm{H}_{203} \mathrm{O}_{12} \mathrm{Si}_{11}{ }^{+}\left([\mathrm{M}+\mathrm{H}]^{+}\right)$2204.2731, found 2204.2684.

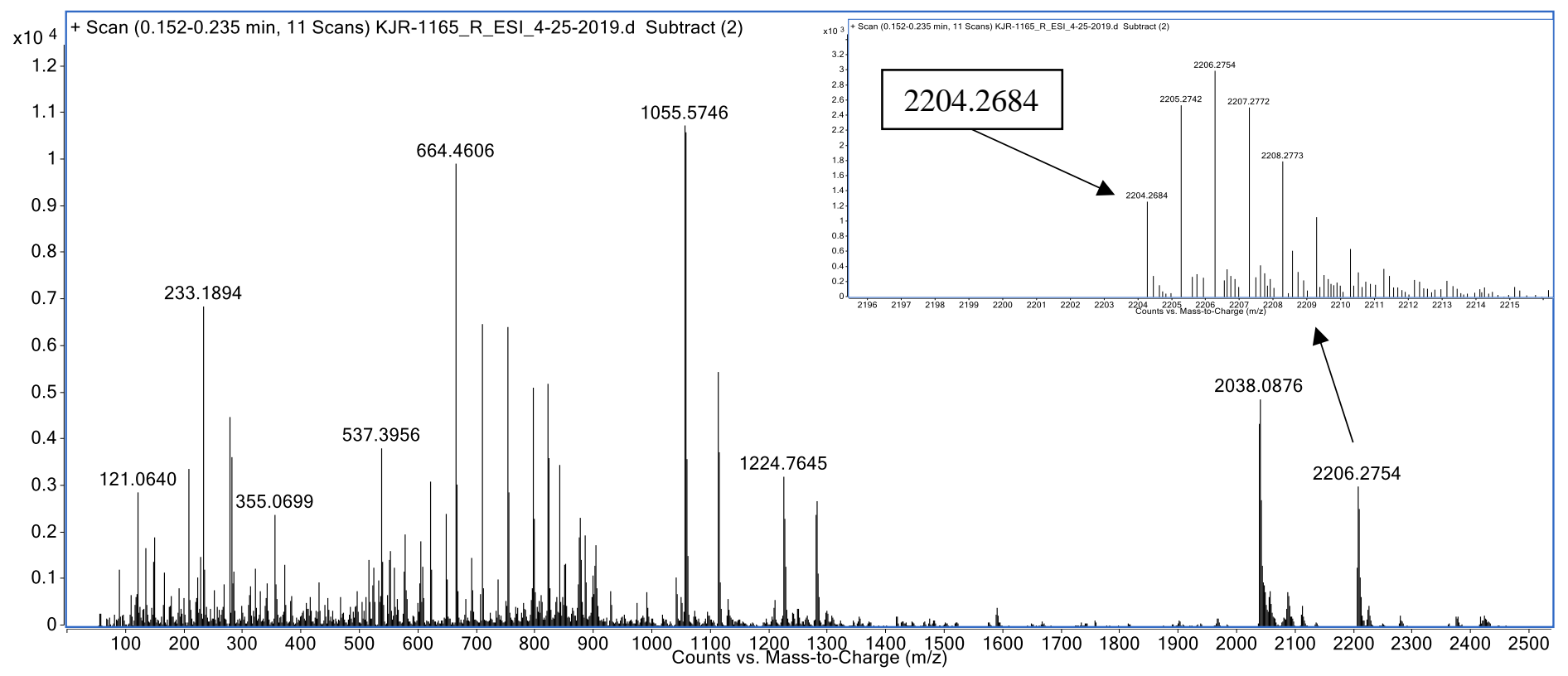



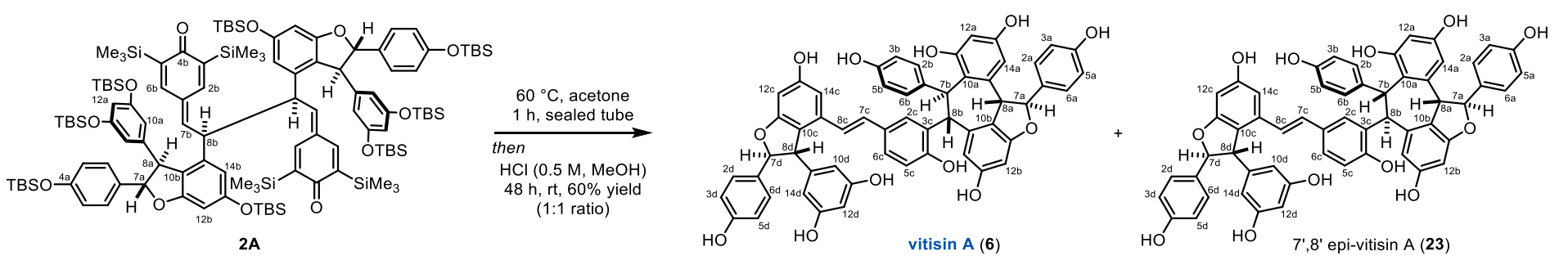

Vitisin A (6) - (4bS,5S,10S,11R)-10-(5-((E)-2-((2S,3S)-3-(3,5-dihydroxyphenyl)-6-hydroxy-2-(4-hydroxyphenyl)-2,3dihydrobenzofuran-4-yl)vinyl)-2-hydroxyphenyl)-5,11-bis(4-hydroxyphenyl)-4b,5,10,11tetrahydrobenzo[6,7]cyclohepta[1,2,3-cd]benzofuran-1,3,8-triol

Quinone-methide dimer 2A (140 mg, $0.066 \mathrm{mmol}$ ) was added to a flame-dried reaction vial charged with a stir bar and dissolved in acetone ( $2.0 \mathrm{~mL}$, HPLC grade). The reaction solution was sparged with a balloon of Ar for 5 minutes and then sealed under argon with parafilm. The reaction was heated to $60{ }^{\circ} \mathrm{C}$ for 1 hour, and upon cooling to ambient temperature, a solution of hydrochloric acid was added $(10 \mathrm{~mL}, 0.5 \mathrm{M}$ in $\mathrm{MeOH})$. The reaction was sealed under an argon atmosphere and allowed to stir at ambient temperature. After 48 hours, the stir bar was removed and the reaction mixture was directly concentrated on the rotovap and the crude residue was purified by flash column chromatography (8\% to $40 \%$ in 5 step increments, 2 column volumes per step, Nonpolar solvent $-\mathrm{CH}_{2} \mathrm{Cl}_{2}$, Polar solvent - 3:1 Acetone/MeOH) to afford a mixture of vitisin $\mathrm{A}(\mathbf{6})$ and its diastereomer $(\mathbf{2 3})$ in a ca. 1:1 ratio $(35.9 \mathrm{mg}, 60 \%$ combined yield). This mixture was further purified by prep-HPLC (10\% to $65 \% \mathrm{MeCN} / \mathrm{H}_{2} \mathrm{O}$ gradient over 1 hour) to afford pure samples of each compound. The ${ }^{1} \mathrm{H}$ and ${ }^{13} \mathrm{C}$ NMR data for vitisin A (6) are consistent with literature reports. ${ }^{7}$

\section{Vitisin A (6):}

${ }^{1} \mathrm{H}$ NMR $\left(700 \mathrm{MHz}\right.$, Acetone- $\left.d_{6}\right) \delta 8.24(\mathrm{~s}, 10 \mathrm{H}, \mathrm{ArOH}), 7.20\left(\mathrm{~d}, J=8.6 \mathrm{~Hz}, 2 \mathrm{H}, \mathrm{C}_{2 / 6 \mathrm{a}}-\underline{\mathrm{H}}\right), 7.15\left(\mathrm{~d}, J=8.5 \mathrm{~Hz}, 2 \mathrm{H}, \mathrm{C}_{2 / 6 \mathrm{~d}}-\right.$ $\underline{\mathrm{H}}), 7.04\left(\mathrm{~d}, J=8.1 \mathrm{~Hz}, 2 \mathrm{H}, \mathrm{C}_{2 / 6 \mathrm{~b}}-\underline{\mathrm{H}}\right), 6.87\left(\mathrm{dd}, J=8.4,2.2 \mathrm{~Hz}, 1 \mathrm{H}, \mathrm{C}_{6 \mathrm{c}}-\underline{\mathrm{H}}\right), 6.84\left(\mathrm{~d}, J=8.6 \mathrm{~Hz}, 2 \mathrm{H}, \mathrm{C}_{3 / 5 \mathrm{a}}-\underline{\mathrm{H}}\right), 6.77(\mathrm{~d}, J=$ $\left.8.6 \mathrm{~Hz}, 2 \mathrm{H}, \mathrm{C}_{3 / 5 \mathrm{~d}}-\underline{\mathrm{H}}\right), 6.69\left(\mathrm{~d}, J=8.4 \mathrm{~Hz}, 1 \mathrm{H}, \mathrm{C}_{5 \mathrm{c}}-\underline{\mathrm{H}}\right), 6.66\left(\mathrm{~d}, J=8.7 \mathrm{~Hz}, 2 \mathrm{H}, \mathrm{C}_{3 / 5 \mathrm{~b}}-\underline{\mathrm{H}}\right), 6.54\left(\mathrm{~d}, J=2.1 \mathrm{~Hz}, 1 \mathrm{H}, \mathrm{C}_{14 \mathrm{a}}-\underline{\mathrm{H}}\right)$, 6.39 (br s, $\left.2 \mathrm{H}, \mathrm{C}_{7 / 8 \mathrm{c}}-\underline{\mathrm{H}}\right), 6.28\left(\mathrm{~d}, J=2.3 \mathrm{~Hz}, 1 \mathrm{H}, \mathrm{C}_{12 \mathrm{a}}-\underline{\mathrm{H}}\right), 6.25\left(\mathrm{~d}, J=2.1 \mathrm{~Hz}, 1 \mathrm{H}, \mathrm{C}_{14 \mathrm{c}}-\underline{\mathrm{H}}\right), 6.21\left(\mathrm{t}, J=2.2 \mathrm{~Hz}, 1 \mathrm{H}, \mathrm{C}_{12 \mathrm{~d}}-\underline{\mathrm{H}}\right)$, $6.16\left(\mathrm{~d}, J=2.2 \mathrm{~Hz}, 2 \mathrm{H}, \mathrm{C}_{10 / 14 \mathrm{~d}}-\underline{\mathrm{H}}\right), 6.09\left(\mathrm{~d}, J=2.0 \mathrm{~Hz}, 1 \mathrm{H}, \mathrm{C}_{2 \mathrm{c}}-\underline{\mathrm{H}}\right), 6.08\left(\mathrm{~d}, J=2.1 \mathrm{~Hz}, 1 \mathrm{H}, \mathrm{C}_{12 \mathrm{~b}}-\underline{\mathrm{H}}\right), 6.05(\mathrm{~d}, J=2.1 \mathrm{~Hz}$, $\left.1 \mathrm{H}, \mathrm{C}_{14 \mathrm{~b}}-\underline{\mathrm{H}}\right), 6.04\left(\mathrm{~d}, J=2.3 \mathrm{~Hz}, 1 \mathrm{H}, \mathrm{C}_{12 \mathrm{c}}-\underline{\mathrm{H}}\right), 5.89\left(\mathrm{~d}, J=11.5 \mathrm{~Hz}, 1 \mathrm{H}, \mathrm{C}_{7 \mathrm{a}}-\underline{\mathrm{H}}\right), 5.49\left(\mathrm{~d}, J=2.4 \mathrm{~Hz}, 1 \mathrm{H}, \mathrm{C}_{8 \mathrm{~b}}-\underline{\mathrm{H}}\right), 5.38(\mathrm{~d}, J$ $\left.=3.9 \mathrm{~Hz}, 1 \mathrm{H}, \mathrm{C}_{7 \mathrm{~b}}-\underline{\mathrm{H}}\right), 5.35\left(\mathrm{~d}, J=5.3 \mathrm{~Hz}, 1 \mathrm{H}, \mathrm{C}_{7 \mathrm{~d}}-\underline{\mathrm{H}}\right), 4.41\left(\mathrm{~d}, J=5.3 \mathrm{~Hz}, 1 \mathrm{H}, \mathrm{C}_{8 \mathrm{~d}}-\underline{\mathrm{H}}\right), 4.24\left(\mathrm{~d}, J=11.5 \mathrm{~Hz}, 1 \mathrm{H}, \mathrm{C}_{8 \mathrm{a}}-\underline{\mathrm{H}}\right)$.

${ }^{13} \mathrm{C}$ NMR $\left(176 \mathrm{MHz}\right.$, Acetone- $\left.d_{6}\right) \delta 162.7,160.5,159.9,159.7,158.9,158.6,158.3,158.0,157.2,156.1,155.2,147.4,142.4$, $141.3,136.7,135.5,134.1,133.0,132.6,131.23,131.17,130.2,129.1,129.0,128.1,123.8,122.9,120.5,120.1,119.0$, $116.3,116.1,115.6,110.2,107.0,105.2,104.7,102.2,101.0,96.7,96.2,94.0,88.6,57.2,49.7,41.4,40.8$.

HRMS (ESI) $m / z$ calculated for $\mathrm{C}_{56} \mathrm{H}_{43} \mathrm{O}_{12}{ }^{+}\left([\mathrm{M}+\mathrm{H}]^{+}\right)$907.2749, found 907.2743. 
${ }^{1} \mathrm{H}$ NMR, $700 \mathrm{MHz}$, Acetone- $d_{6}$, Compound 6

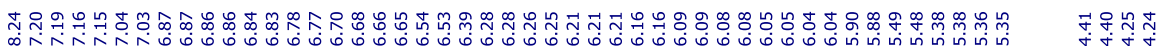
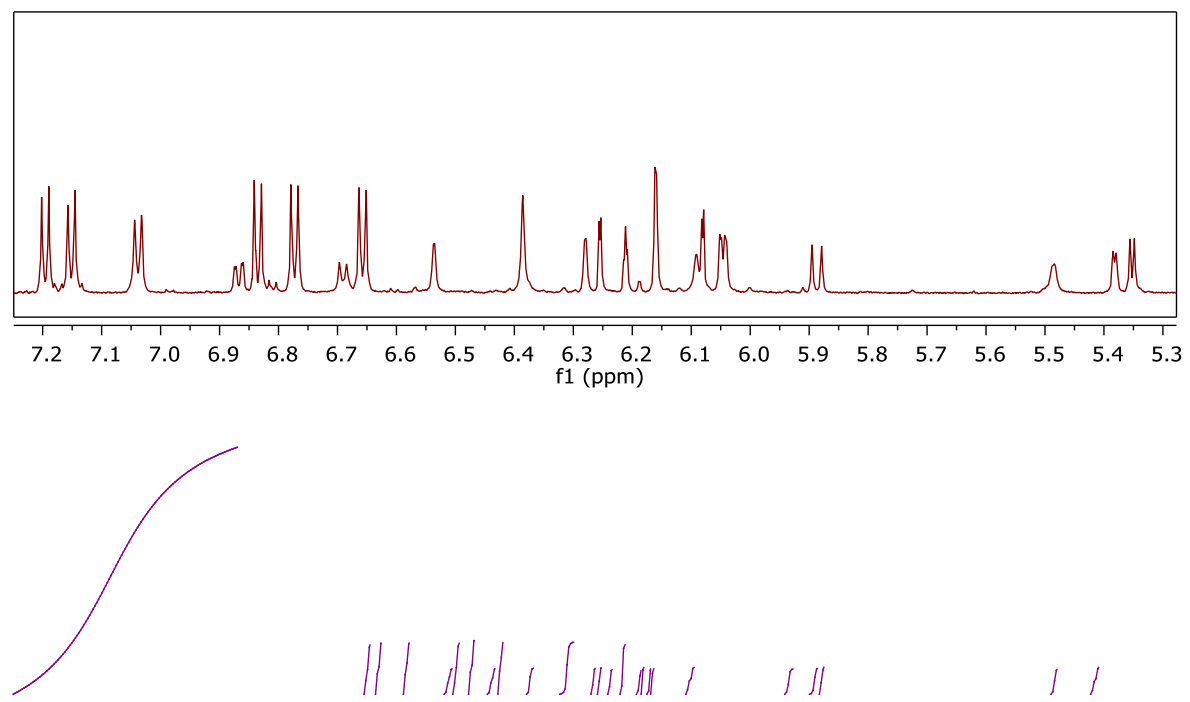

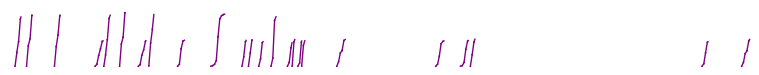

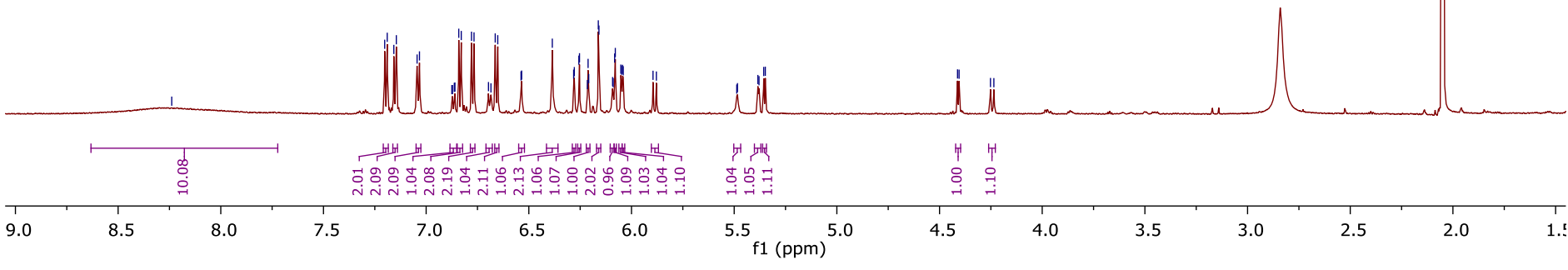

${ }^{13} \mathrm{C}$ NMR, $176 \mathrm{MHz}$, Acetone- $d_{6}$, Compound 6 (full spectrum)

V|r

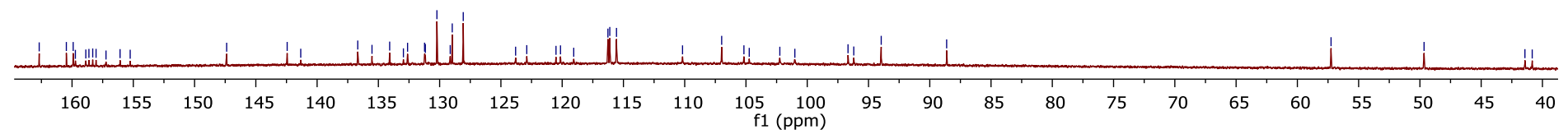




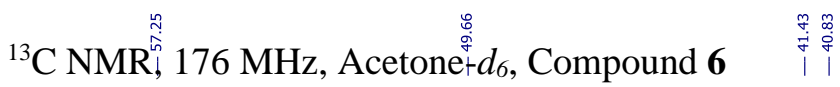

$(20-60 \mathrm{ppm})$
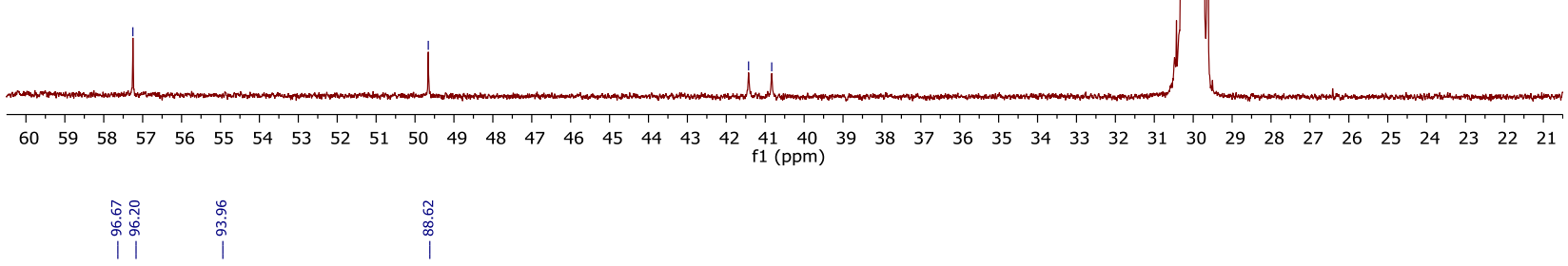

${ }^{13} \mathrm{C}$ NMR, $176 \mathrm{MHz}$, Acetone- $d_{6}$, Compound 6

$(60-100 \mathrm{ppm})$

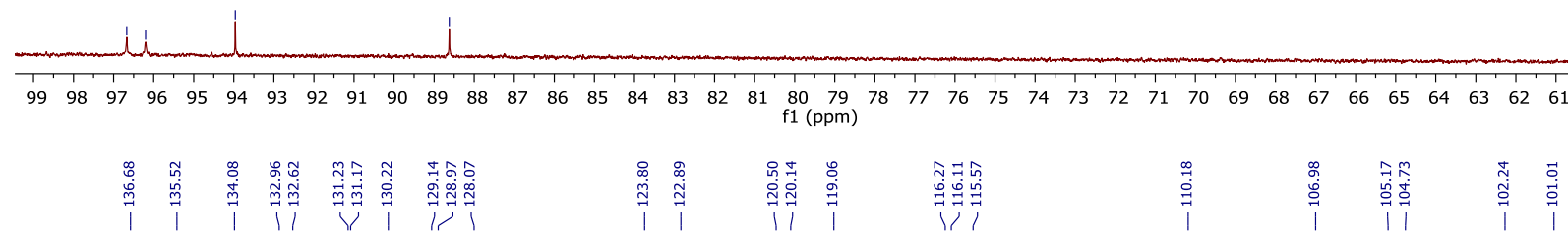

${ }^{13} \mathrm{C}$ NMR, $176 \mathrm{MHz}$, Acetone- $d_{6}$, Compound 6

$(100-140 \mathrm{ppm})$

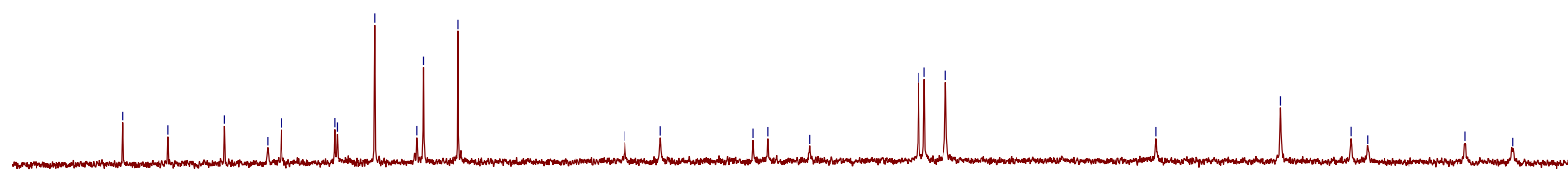

139138137136135134133132131130129128127126125124123122121120119118117116115114113112111110109108107106105104103102101100 f1 (ppm)

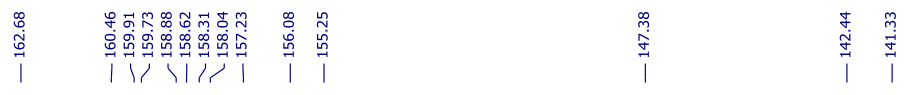

${ }^{13} \mathrm{C}$ NMR, $176 \mathrm{MHz}$, Acetone- $d_{6}$, Compound 6

$(140-180 \mathrm{ppm})$

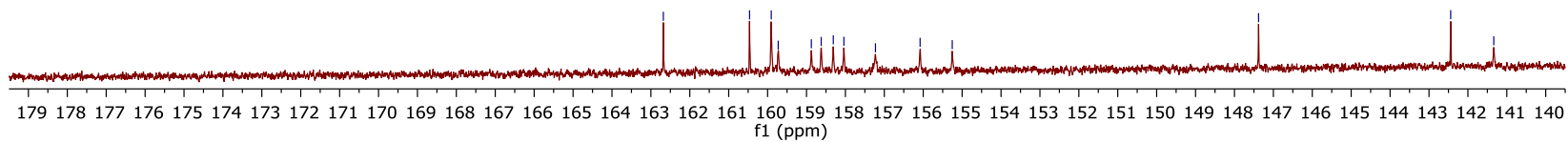


${ }^{1} \mathrm{H}$ NMR $\left(500 \mathrm{MHz}\right.$, Acetone- $\left.d_{6}\right) \delta 8.28(\mathrm{~s}, 10 \mathrm{H}), 7.17\left(\mathrm{~d}, J=8.6 \mathrm{~Hz}, 2 \mathrm{H}, \mathrm{C}_{2 / 6 \mathrm{a}}-\underline{\mathrm{H}}\right), 7.14\left(\mathrm{~d}, J=8.6 \mathrm{~Hz}, 2 \mathrm{H}, \mathrm{C}_{2 / 6 \mathrm{~d}}-\underline{\mathrm{H}}\right), 7.04$ $\left(\mathrm{d}, J=8.3 \mathrm{~Hz}, 2 \mathrm{H}, \mathrm{C}_{2 / 6 \mathrm{~b}}-\underline{\mathrm{H}}\right), 6.82\left(\mathrm{dd}, J=8.5,2.2 \mathrm{~Hz}, 1 \mathrm{H}, \mathrm{C}_{6 \mathrm{c}}-\underline{\mathrm{H}}\right), 6.81\left(\mathrm{~d}, J=8.5 \mathrm{~Hz}, 2 \mathrm{H}, \mathrm{C}_{3 / 5 \mathrm{a}}-\underline{\mathrm{H}}\right), 6.77(\mathrm{~d}, J=8.5 \mathrm{~Hz}$ $\left.2 \mathrm{H}, \mathrm{C}_{3 / 5 \mathrm{~d}}-\underline{\mathrm{H}}\right), 6.68\left(\mathrm{~d}, J=8.4 \mathrm{~Hz}, 1 \mathrm{H}, \mathrm{C}_{5 \mathrm{c}}-\underline{\mathrm{H}}\right), 6.66\left(\mathrm{~d}, J=8.6 \mathrm{~Hz}, 2 \mathrm{H}, \mathrm{C}_{3 / 5 \mathrm{~b}}-\underline{\mathrm{H}}\right), 6.56\left(\mathrm{~d}, J=2.1 \mathrm{~Hz}, 1 \mathrm{H}, \mathrm{C}_{14 \mathrm{a}}-\underline{\mathrm{H}}\right), 6.42(\mathrm{~d}$, $\left.J=16.3 \mathrm{~Hz}, 1 \mathrm{H}, \mathrm{C}_{7 \mathrm{c}}-\underline{\mathrm{H}}\right), 6.36\left(\mathrm{~d}, J=16.4 \mathrm{~Hz}, 1 \mathrm{H}, \mathrm{C}_{8 \mathrm{c}}-\underline{\mathrm{H}}\right), 6.31\left(\mathrm{~d}, J=2.3 \mathrm{~Hz}, 1 \mathrm{H}, \mathrm{C}_{14 \mathrm{c}}-\underline{\mathrm{H}}\right), 6.28\left(\mathrm{t}, J=2.2 \mathrm{~Hz}, 1 \mathrm{H}, \mathrm{C}_{12 \mathrm{~d}}-\right.$ $\underline{\mathrm{H}}), 6.25\left(\mathrm{~d}, J=2.0 \mathrm{~Hz}, 1 \mathrm{H}, \mathrm{C}_{12 \mathrm{a}}-\underline{\mathrm{H}}\right), 6.19\left(\mathrm{~d}, J=2.2 \mathrm{~Hz}, 2 \mathrm{H}, \mathrm{C}_{10 / 14 \mathrm{~d}}-\underline{\mathrm{H}}\right), 6.12\left(\mathrm{~d}, J=2.1 \mathrm{~Hz}, 1 \mathrm{H}, \mathrm{C}_{2 \mathrm{c}}-\underline{\mathrm{H}}\right), 6.09(\mathrm{~d}, J=2.2$ $\left.\mathrm{Hz}, 1 \mathrm{H}, \mathrm{C}_{12 \mathrm{~b}}-\underline{\mathrm{H}}\right), 6.08\left(\mathrm{~d}, J=2.3 \mathrm{~Hz}, 1 \mathrm{H}, \mathrm{C}_{14 \mathrm{~b}}-\underline{\mathrm{H}}\right), 6.00\left(\mathrm{~d}, J=2.2 \mathrm{~Hz}, 1 \mathrm{H}, \mathrm{C}_{12 \mathrm{c}}-\underline{\mathrm{H}}\right), 5.90\left(\mathrm{~d}, J=11.5 \mathrm{~Hz}, 1 \mathrm{H}, \mathrm{C}_{7 \mathrm{a}}-\underline{\mathrm{H}}\right), 5.50$ $\left(\mathrm{d}, J=3.9 \mathrm{~Hz}, 1 \mathrm{H}, \mathrm{C}_{8 \mathrm{~b}}-\underline{\mathrm{H}}\right), 5.36\left(\mathrm{~d}, J=4.6 \mathrm{~Hz}, 1 \mathrm{H}, \mathrm{C}_{7 \mathrm{~b}}-\underline{\mathrm{H}}\right), 5.34\left(\mathrm{~d}, J=3.8 \mathrm{~Hz}, 1 \mathrm{H}, \mathrm{C}_{7 \mathrm{~d}}-\underline{\mathrm{H}}\right), 4.39\left(\mathrm{~d}, J=4.6 \mathrm{~Hz}, 1 \mathrm{H}, \mathrm{C}_{8 \mathrm{~d}-}\right.$ $\underline{\mathrm{H}}), 4.25\left(\mathrm{~d}, J=11.5 \mathrm{~Hz}, 1 \mathrm{H}, \mathrm{C}_{8 \mathrm{a}}-\underline{\mathrm{H}}\right)$.

${ }^{13} \mathrm{C}$ NMR $\left(126 \mathrm{MHz}\right.$, Acetone- $\left.d_{6}\right) \delta 162.67,160.46,159.94,159.71,158.81,158.60,158.27,158.05,157.29,156.07,155.21$, $147.91,142.49,141.39,136.48,135.52,134.17,132.95,132.20,131.23,130.98,130.24,129.11,128.98,128.00,124.29$, $122.87,120.47,120.12,119.19,116.26,116.11,115.57,115.44,110.14,106.95,105.13,104.59,102.25,100.95,96.67$, $96.18,93.92,88.61,56.96,49.69,41.27,40.93$.

HRMS (ESI) $m / z$ calculated for $\mathrm{C}_{56} \mathrm{H}_{43} \mathrm{O}_{12}{ }^{+}\left([\mathrm{M}+\mathrm{H}]^{+}\right)$907.2749, found 907.2749.

${ }^{1} \mathrm{H}$ NMR, $500 \mathrm{MHz}$, Acetone- $d_{6}$, Compound 23

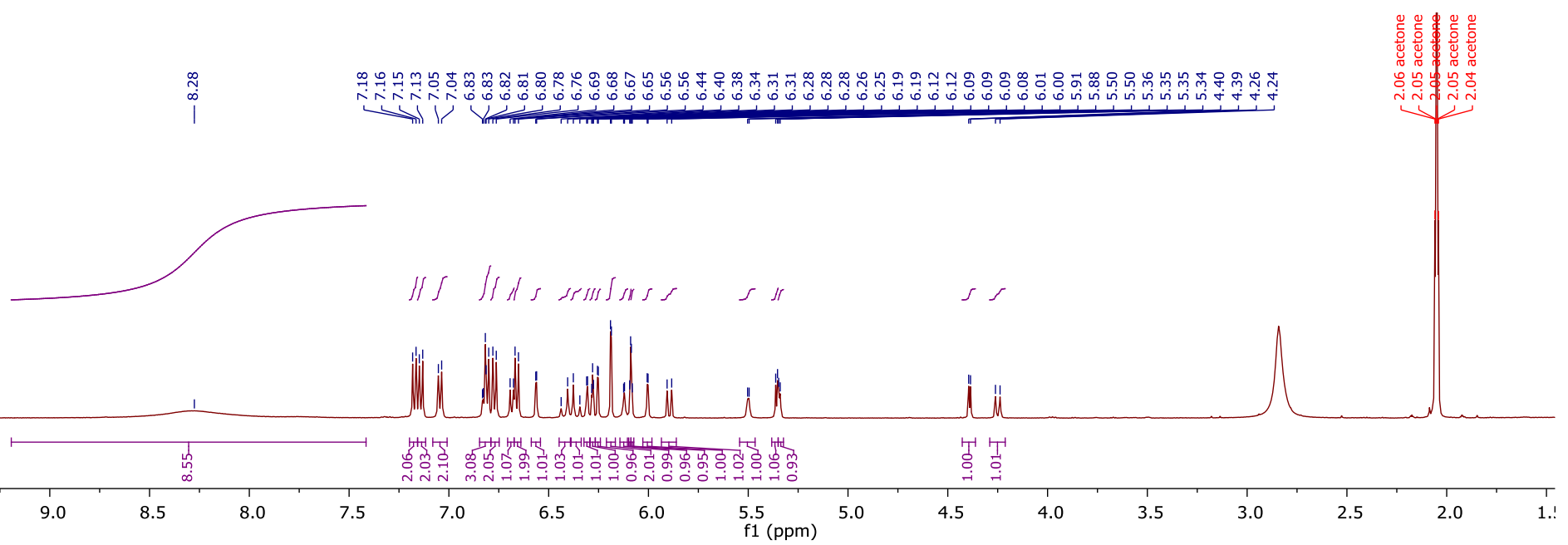

${ }^{13} \mathrm{C}$ NMR, $500 \mathrm{MHz}$, Acetone- $d_{6}$, Compound 23

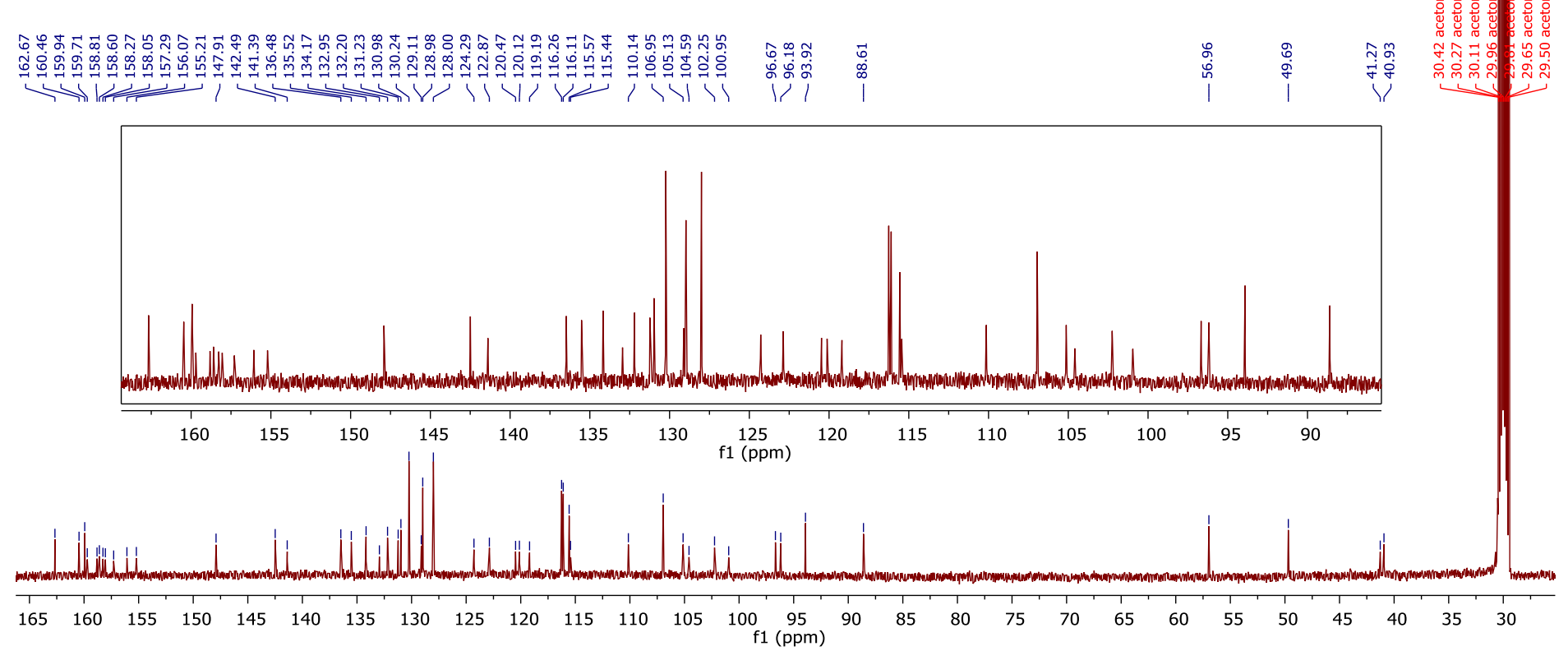




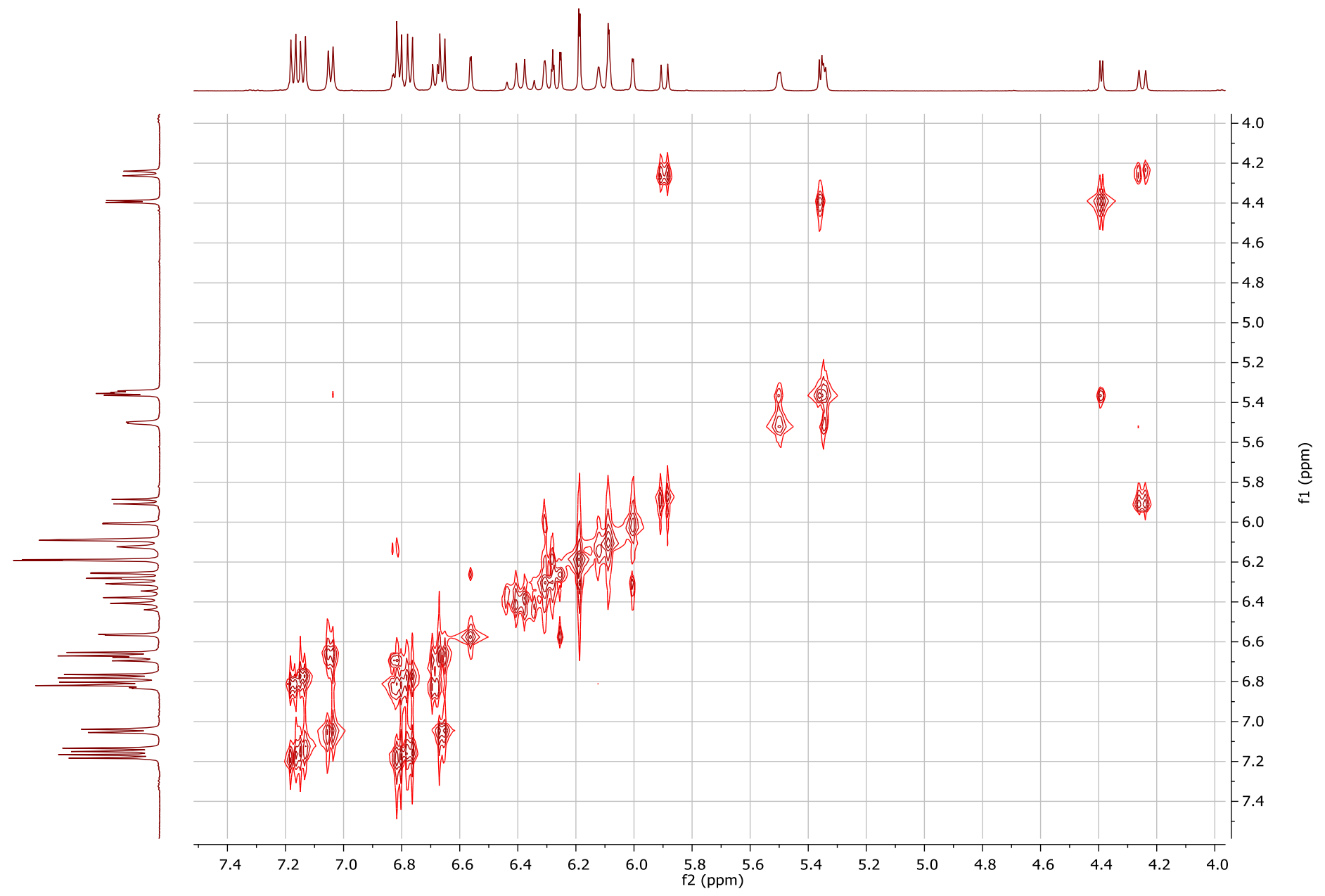


Table S2. Comparison of literature and observed ${ }^{1} \mathrm{H}$ NMR data for vitisin A (6).

\begin{tabular}{ccc} 
& Literature $^{7}$ & Observed \\
\hline Assignment & $500 \mathrm{MHz}$, Acetone- $d_{6}$ & $700 \mathrm{MHz}$, Acetone- $d_{6}$ \\
\hline $2 / 6 \mathrm{a}$ & $7.22(\mathrm{~d}, J=8.5 \mathrm{~Hz})$ & $7.20(\mathrm{~d}, J=8.6 \mathrm{~Hz}, 2 \mathrm{H})$ \\
$3 / 5 \mathrm{a}$ & $6.86(\mathrm{~d}, J=8.5 \mathrm{~Hz})$ & $6.84(\mathrm{~d}, J=8.6 \mathrm{~Hz}, 2 \mathrm{H})$ \\
$7 \mathrm{a}$ & $5.91(\mathrm{~d}, J=10.5 \mathrm{~Hz})$ & $5.89(\mathrm{~d}, J=11.5 \mathrm{~Hz}, 1 \mathrm{H})$ \\
$8 \mathrm{a}$ & $4.26(\mathrm{~d}, J=10.5 \mathrm{~Hz})$ & $4.24(\mathrm{~d}, J=11.5 \mathrm{~Hz}, 1 \mathrm{H})$ \\
$12 \mathrm{a}$ & $6.28(\mathrm{~d}, J=2.0 \mathrm{~Hz})$ & $6.28(\mathrm{~d}, J=2.3 \mathrm{~Hz}, 1 \mathrm{H})$, \\
$14 \mathrm{a}$ & $6.55(\mathrm{~d}, J=2.0 \mathrm{~Hz})$ & $6.54(\mathrm{~d}, J=2.1 \mathrm{~Hz}, 1 \mathrm{H})$ \\
$2 / 6 \mathrm{~b}$ & $7.06(\mathrm{~d}, J=8.5 \mathrm{~Hz})$ & $7.04(\mathrm{~d}, J=8.1 \mathrm{~Hz}, 2 \mathrm{H})$ \\
$3 / 5 \mathrm{~b}$ & $6.68(\mathrm{~d}, J=8.5 \mathrm{~Hz})$ & $6.66(\mathrm{~d}, J=8.7 \mathrm{~Hz}, 2 \mathrm{H})$ \\
$7 \mathrm{~b}$ & $5.41(\mathrm{~d}, J=3.0 \mathrm{~Hz})$ & $5.38(\mathrm{~d}, J=3.9 \mathrm{~Hz}, 1 \mathrm{H})$ \\
$8 \mathrm{~b}$ & $5.50(\mathrm{~d}, J=3.0 \mathrm{~Hz})$ & $5.49(\mathrm{~d}, J=2.4 \mathrm{~Hz}, 1 \mathrm{H})$ \\
$12 \mathrm{~b}$ & $6.11(\mathrm{~d}, J=2.0 \mathrm{~Hz})$ & $6.08(\mathrm{~d}, J=2.1 \mathrm{~Hz}, 1 \mathrm{H})$ \\
$14 \mathrm{~b}$ & $6.08(\mathrm{~d}, J=2.0 \mathrm{~Hz})$ & $6.05(\mathrm{~d}, J=2.1 \mathrm{~Hz}, 1 \mathrm{H})$ \\
$2 \mathrm{c}$ & $6.12(\mathrm{~d}, J=2.0 \mathrm{~Hz})$ & $6.09(\mathrm{~d}, J=2.0 \mathrm{~Hz}, 1 \mathrm{H})$ \\
$5 \mathrm{c}$ & $6.71(\mathrm{~d}, J=8.5 \mathrm{~Hz})$ & $6.69(\mathrm{~d}, J=8.4 \mathrm{~Hz}, 1 \mathrm{H})$ \\
$6 \mathrm{c}$ & $6.41(\mathrm{dbr})$ & $6.87(\mathrm{dd}, J=8.4,2.2 \mathrm{~Hz})$ \\
$7 \mathrm{c}$ & $6.41(\mathrm{br})$ & $6.39(\mathrm{br}, 2 \mathrm{H})$ \\
$8 \mathrm{c}$ & $6.06(\mathrm{~d}, J=2.0 \mathrm{~Hz})$ & $6.39(\mathrm{br}, 2 \mathrm{H})$ \\
$12 \mathrm{c}$ & $6.27(\mathrm{~d}, J=2.0 \mathrm{~Hz})$ & $6.04(\mathrm{~d}, J=2.3 \mathrm{~Hz}, 1 \mathrm{H})$ \\
$14 \mathrm{c}$ & $7.17(\mathrm{~d}, J=8.5 \mathrm{~Hz})$ & $6.25(\mathrm{~d}, J=2.1,1 \mathrm{H})$ \\
$2 / 6 \mathrm{~d}$ & $6.80(\mathrm{~d}, J=8.5 \mathrm{~Hz})$ & $7.15(\mathrm{~d}, J=8.5 \mathrm{~Hz}, 2 \mathrm{H})$ \\
$3 / 5 \mathrm{~d}$ & $5.38(\mathrm{~d}, J=5.0 \mathrm{~Hz})$ & $6.77(\mathrm{~d}, J=8.6 \mathrm{~Hz}, 2 \mathrm{H})$ \\
$7 \mathrm{~d}$ & $4.43(\mathrm{~d}, J=5.0 \mathrm{~Hz})$ & $5.35(\mathrm{~d}, J=5.3 \mathrm{~Hz}, 1 \mathrm{H})$ \\
$8 \mathrm{~d}$ & $6.19(\mathrm{~d}, J=2.0 \mathrm{~Hz})$ & $4.41(\mathrm{~d}, J=5.3 \mathrm{~Hz}, 1 \mathrm{H})$ \\
$10 / 14 \mathrm{~d}$ & $6.24(\mathrm{t}, J=2.0 \mathrm{~Hz})$ & $6.16(\mathrm{~d}, J=2.2 \mathrm{~Hz}, 2 \mathrm{H})$ \\
$12 \mathrm{~d}$ & $6.21(\mathrm{t}, J=2.2 \mathrm{~Hz}, 1 \mathrm{H})$ \\
\hline
\end{tabular}

Note: The $7 \mathrm{c} / 8 \mathrm{c}$ signals were anticipated to appear as an AB multiplet characteristic of a stilbene; instead, a broad singlet is observed, which presumably results from the doublets overlapping with each other such that the peripheral peak of each signal is indistinguishable from the baseline. 
Table S3. Comparison of literature and observed ${ }^{13} \mathrm{C}$ NMR data for vitisin A (6).

\begin{tabular}{|c|c|c|}
\hline & Literature $^{7}$ & Observed \\
\hline Assignment & $125 \mathrm{MHz}$, Acetone- $d_{6}$ & $176 \mathrm{MHz}$, Acetone- $d_{6}$ \\
\hline $1 \mathrm{a}$ & 131.3 & 131.2 \\
\hline $2 / 6 \mathrm{a}$ & 128.3 & 128.1 \\
\hline $3 / 5 \mathrm{a}$ & 116.5 & 116.3 \\
\hline $4 \mathrm{a}$ & 158.5 & 158.3 \\
\hline $7 \mathrm{a}$ & 88.8 & 88.6 \\
\hline $8 a$ & 57.4 & 57.2 \\
\hline $9 a$ & 142.6 & 142.4 \\
\hline $10 \mathrm{a}$ & 120.6 & 120.5 \\
\hline $11 \mathrm{a}$ & 158.3 & 158.0 \\
\hline $12 \mathrm{a}$ & 96.9 & 96.7 \\
\hline $13 \mathrm{a}$ & 156.2 & 156.1 \\
\hline $14 \mathrm{a}$ & 104.9 & 104.7 \\
\hline $1 \mathrm{~b}$ & 135.7 & 135.5 \\
\hline $2 / 6 b$ & 129.2 & 129.0 \\
\hline $3 / 5 b$ & 115.8 & 115.6 \\
\hline $4 b$ & 155.5 & 155.2 \\
\hline $7 b$ & 41.0 & 40.8 \\
\hline $8 b$ & 41.6 & 41.4 \\
\hline $9 b$ & 141.5 & 141.3 \\
\hline $10 \mathrm{~b}$ & 120.5 & 120.1 \\
\hline $11 \mathrm{~b}$ & 157.2 & 157.2 \\
\hline $12 \mathrm{~b}$ & 96.4 & 96.2 \\
\hline $13 b$ & 159.1 & 158.9 \\
\hline $14 \mathrm{~b}$ & 110.4 & 110.2 \\
\hline $1 \mathrm{c}$ & 129.2 & 129.1 \\
\hline $2 c$ & 132.7 & 132.6 \\
\hline $3 \mathrm{c}$ & 136.9 & 136.7 \\
\hline $4 c$ & 160.0 & 159.9 \\
\hline $5 c$ & 115.8 & 115.6 \\
\hline $6 \mathrm{c}$ & 124.1 & 123.8 \\
\hline $7 \mathrm{c}$ & 131.4 & 131.2 \\
\hline $8 \mathrm{c}$ & 123.0 & 122.9 \\
\hline $9 \mathrm{c}$ & 134.2 & 134.1 \\
\hline $10 \mathrm{c}$ & 119.4 & 119.1 \\
\hline $11 \mathrm{c}$ & 162.8 & 162.7 \\
\hline $12 \mathrm{c}$ & 101.3 & 101.0 \\
\hline $13 c$ & 159.8 & 159.7 \\
\hline $14 \mathrm{c}$ & 105.3 & 105.2 \\
\hline $1 \mathrm{~d}$ & 133.1 & 133.0 \\
\hline $2 / 6 \mathrm{~d}$ & 130.4 & 130.2 \\
\hline $3 / 5 \mathrm{~d}$ & 116.4 & 116.1 \\
\hline $4 d$ & 158.8 & 158.6 \\
\hline $7 \mathrm{~d}$ & 94.1 & 94.0 \\
\hline $8 d$ & 49.8 & 49.7 \\
\hline $9 \mathrm{~d}$ & 147.6 & 147.4 \\
\hline $10 / 14 d$ & 107.2 & 107.0 \\
\hline $11 / 13 \mathrm{~d}$ & 160.6 & 160.5 \\
\hline $12 \mathrm{~d}$ & 102.5 & 102.2 \\
\hline
\end{tabular}



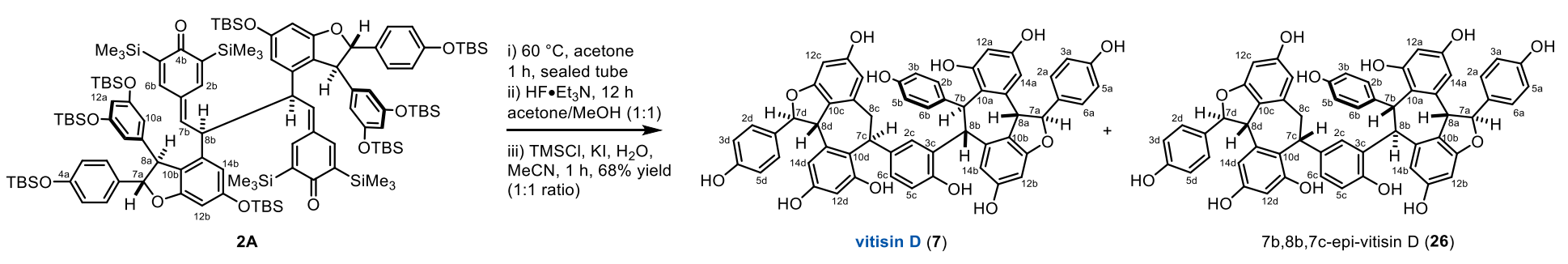

Vitisin D (7) - (1S,7S,11bS)-7-(4-hydroxy-3-((4bS,5S,10S,11R)-1,3,8-trihydroxy-5,11-bis(4-hydroxyphenyl)-
4b,5,10,11-tetrahydrobenzo[6,7]cyclohepta[1,2,3-cd]benzofuran-10-yl)phenyl)-1-(4-hydroxyphenyl)-1,6,7,11b-
tetrahydrobenzo[6,7]cyclohepta[1,2,3-cd]benzofuran-4,8,10-triol

Quinone-methide dimer 2A (100 mg, $0.047 \mathrm{mmol})$ was added to a flame-dried reaction vial charged with a stir bar and dissolved in acetone (1.5 mL, HPLC grade). The reaction solution was sparged with a balloon of Ar for 5 minutes and then sealed under argon with parafilm. The reaction was heated to $60{ }^{\circ} \mathrm{C}$ for 1 hour, and upon cooling to ambient temperature, a solution of HF-triethylamine was added $(0.48 \mathrm{~mL}, 2.94 \mathrm{mmol})$ followed by $\mathrm{MeOH}(1.5 \mathrm{~mL}$, HPLC grade). The reaction was sealed under an argon atmosphere and allowed to stir at ambient temperature. After 16 hours, the stir bar was removed, and the reaction mixture was added to a separatory funnel containing sat. aq. sodium bicarbonate $(\sim 20 \mathrm{~mL})$. The aqueous layer was extracted with portion of EtOAc $(3 \times 15 \mathrm{~mL})$, and the combined organic layers were washed with brine, dried over $\mathrm{MgSO}_{4}$, and concentrated under reduced pressure. The crude material was consistent with cleavage of the 8 TBS ethers, so it was directly carried forward for protodesilylation. The material was dissolved in MeCN (1.0 $\mathrm{mL}$ ) in a reaction vial, and potassium iodide $(25 \mathrm{mg}, 0.15 \mathrm{mmol})$, TMSCl $(19.4 \mu \mathrm{L}, 0.15 \mathrm{mmol})$, and water $(3 \mu \mathrm{L}, 0.16 \mathrm{mmol})$ were added. The reaction was allowed to stir at room temperature for 1 hour, at which point it was quenched with triethylamine $(0.5 \mathrm{~mL})$ and directly concentrated on the rotovap. The crude residue was purified by flash column chromatography (8\% to $40 \%$ in 5 step increments, 2 column volumes per step, Nonpolar solvent $-\mathrm{CH}_{2} \mathrm{Cl}_{2}$, Polar solvent - 3:1 Acetone/MeOH) to afford a mixture of vitisin D (7) and its diastereomer (26) in a ca. 1:1 ratio (28.8 mg, 68\% combined yield). This mixture was further purified by prep-HPLC (10\% to $65 \% \mathrm{MeCN} / \mathrm{H}_{2} \mathrm{O}$ gradient over 1 hour) to afford pure samples of each compound. The ${ }^{1} \mathrm{H}$ and ${ }^{13} \mathrm{C}$ NMR data for vitisin D (7) are consistent with literature reports. ${ }^{8,9}$

\section{Vitisin D (7):}

${ }^{1} \mathrm{H}$ NMR $\left(700 \mathrm{MHz}\right.$, Methanol- $\left.d_{4}\right) \delta 7.11\left(\mathrm{~d}, J=8.2 \mathrm{~Hz}, 2 \mathrm{H}, \mathrm{C}_{2 / 6 \mathrm{a}}-\underline{\mathrm{H}}\right), 7.06\left(\mathrm{~d}, J=8.2 \mathrm{~Hz}, 2 \mathrm{H}, \mathrm{C}_{2 / 6 \mathrm{~d}}-\underline{\mathrm{H}}\right), 6.94(\mathrm{~d}, J=8.2 \mathrm{~Hz}$, $\left.2 \mathrm{H}, \mathrm{C}_{2 / 6 \mathrm{~b}}-\underline{\mathrm{H}}\right), 6.83\left(\mathrm{~d}, J=8.1 \mathrm{~Hz}, 2 \mathrm{H}, \mathrm{C}_{3 / 5 \mathrm{~d}}-\underline{\mathrm{H}}\right), 6.70\left(\mathrm{~d}, J=8.2 \mathrm{~Hz}, 2 \mathrm{H}, \mathrm{C}_{3 / 5 \mathrm{a}}-\underline{\mathrm{H}}\right), 6.57\left(\mathrm{~d}, J=8.3 \mathrm{~Hz}, 2 \mathrm{H}, \mathrm{C}_{3 / 5 \mathrm{~b}}-\underline{\mathrm{H}}\right), 6.51(\mathrm{~d}$, $\left.J=8.4 \mathrm{~Hz}, 1 \mathrm{H}, \mathrm{C}_{6 \mathrm{c}}-\underline{\mathrm{H}}\right), 6.49\left(\mathrm{~d}, J=8.5 \mathrm{~Hz}, 1 \mathrm{H}, \mathrm{C}_{5 \mathrm{c}}-\underline{\mathrm{H}}\right), 6.15\left(\mathrm{~d}, J=2.2 \mathrm{~Hz}, 1 \mathrm{H}, \mathrm{C}_{12 \mathrm{~d}}-\underline{\mathrm{H}}\right), 6.03\left(\mathrm{~s}, 1 \mathrm{H}, \mathrm{C}_{14 \mathrm{c}}-\underline{\mathrm{H}}\right), 6.02(\mathrm{~s}, 1 \mathrm{H}$, $\left.\mathrm{C}_{12 \mathrm{c}}-\underline{\mathrm{H}}\right), 6.00\left(\mathrm{~s}, 1 \mathrm{H}, \mathrm{C}_{12 \mathrm{~b}}-\underline{\mathrm{H}}\right), 5.98\left(\mathrm{~d}, J=2.1 \mathrm{~Hz}, 1 \mathrm{H}, \mathrm{C}_{14 \mathrm{~d}}-\underline{\mathrm{H}}\right), 5.96\left(\mathrm{~s}, 1 \mathrm{H}, \mathrm{C}_{2 \mathrm{c}}-\underline{\mathrm{H}}\right), 5.87\left(\mathrm{~s}, 1 \mathrm{H}, \mathrm{C}_{14 \mathrm{a}}-\underline{\mathrm{H}}\right), 5.84(\mathrm{~d}, J=2.9$ $\left.\mathrm{Hz}, 1 \mathrm{H}, \mathrm{C}_{12 \mathrm{a}}-\underline{\mathrm{H}}\right), 5.83\left(\mathrm{~s}, 1 \mathrm{H}, \mathrm{C}_{14 \mathrm{~b}}-\underline{\mathrm{H}}\right), 5.73\left(\mathrm{~d}, J=11.9 \mathrm{~Hz}, 1 \mathrm{H}, \mathrm{C}_{7 \mathrm{a}}-\underline{\mathrm{H}}\right), 5.56\left(\mathrm{~d}, J=4.2 \mathrm{~Hz}, 1 \mathrm{H}, \mathrm{C}_{7 \mathrm{~b}}-\underline{\mathrm{H}}\right), 5.55(\mathrm{~d}, J=11.1$ $\left.\mathrm{Hz}, 1 \mathrm{H}, \mathrm{C}_{7 \mathrm{~d}}-\underline{\mathrm{H}}\right), 5.24\left(\mathrm{~d}, J=4.3 \mathrm{~Hz}, 1 \mathrm{H}, \mathrm{C}_{8 \mathrm{~b}}-\underline{\mathrm{H}}\right), 4.68\left(\mathrm{t}, J=4.0 \mathrm{~Hz}, 1 \mathrm{H}, \mathrm{C}_{7 \mathrm{c}}-\underline{\mathrm{H}}\right), 3.98\left(\mathrm{~d}, J=11.9 \mathrm{~Hz}, 1 \mathrm{H}, \mathrm{C}_{8 \mathrm{a}}-\underline{\mathrm{H}}\right), 3.91(\mathrm{~d}$, $\left.J=11.2 \mathrm{~Hz}, 1 \mathrm{H}, \mathrm{C}_{8 \mathrm{~d}}-\underline{\mathrm{H}}\right), 3.11\left(\mathrm{dd}, J=18.0,3.8 \mathrm{~Hz}, 1 \mathrm{H}, \mathrm{C}_{8 \mathrm{c}}-\underline{\mathrm{H}}\right), 2.90\left(\mathrm{dd}, J=17.8,3.7 \mathrm{~Hz}, 1 \mathrm{H}, \mathrm{C}_{8 \mathrm{c}}-\underline{\mathrm{H}}\right)$.

${ }^{13} \mathrm{C}$ NMR $\left(176 \mathrm{MHz}\right.$, Methanol- $\left.d_{4}\right) \delta 160.14,160.12,158.8,158.4,158.2,157.6,156.7,156.1,155.6,153.2,143.2,141.7$, 141.5, 138.3, 136.3, 134.6, 132.7, 131.8, 131.4, 131.3, 130.7, 130.6, 129.4, 125.1, 123.3, 121.05, 120.98, 119.6, 116.4, $116.1,115.4,114.2,110.5,108.8,106.6,105.1,101.6,101.1,96.2,95.9,89.2,89.0,49.4,49.3,43.9,40.5,36.2,33.6$. 
${ }^{1} \mathrm{H}$ NMR, $700 \mathrm{MHz}$, Methanol- $d_{4}$, Compound 7

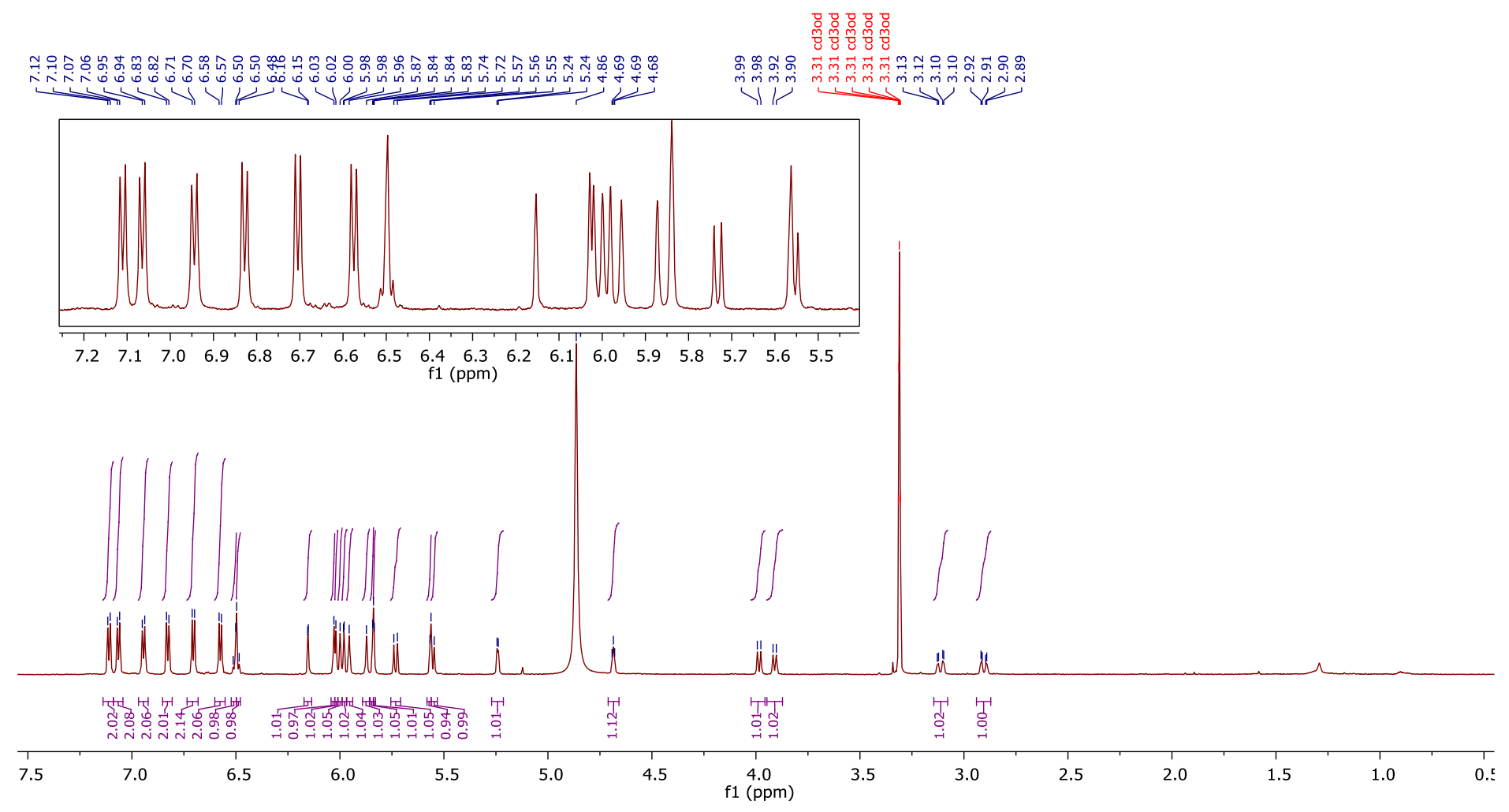

${ }^{13} \mathrm{C}$ NMR, $176 \mathrm{MHz}$, Methanol- $d_{4}$, Compound 7 (full spectrum)

V|
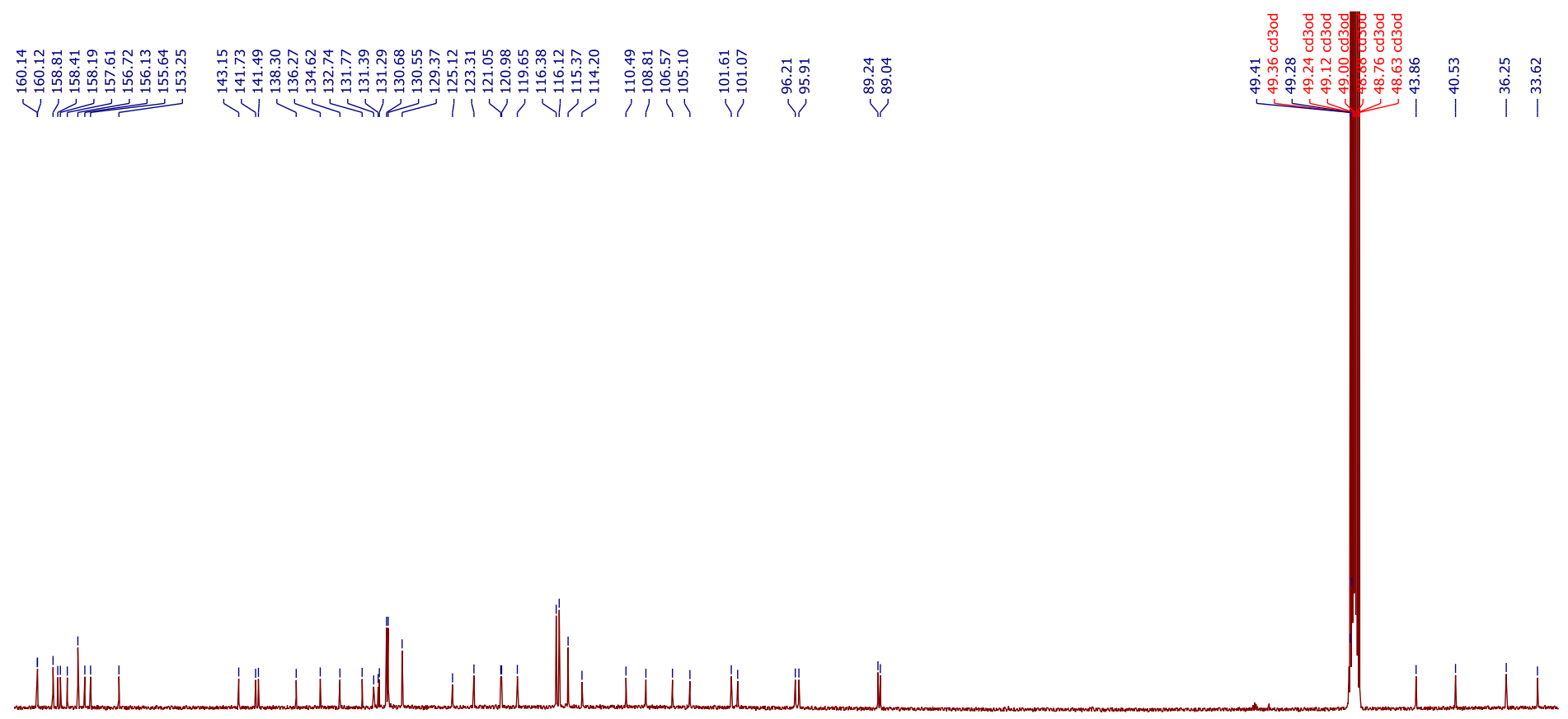

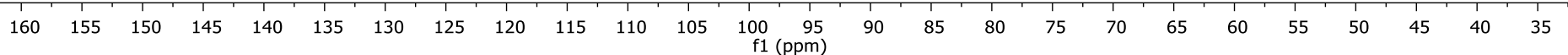


${ }^{13} \mathrm{C}$ NMR, $176 \mathrm{MHz}$, Methanol- $d_{4}$, Compound $7(30-60$ ppm)

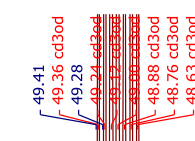

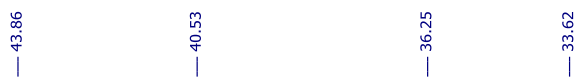

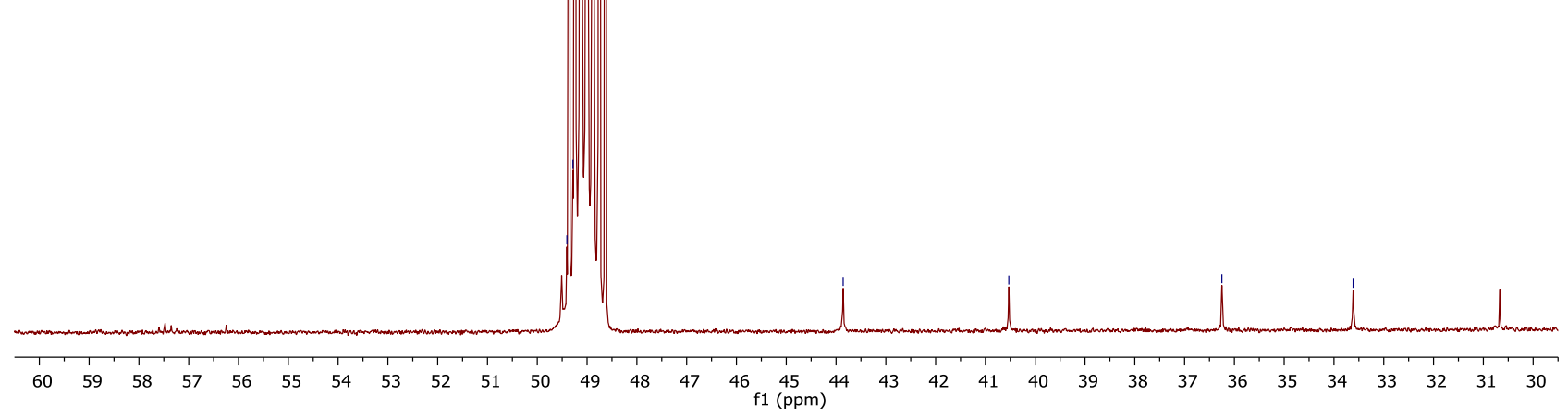

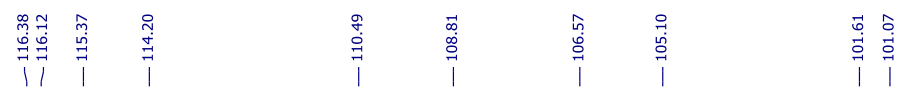

तี कू

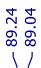

${ }^{13} \mathrm{C}$ NMR, $176 \mathrm{MHz}$, Methanol- $d_{4}$, Compound 7

$(90-118 \mathrm{ppm})$

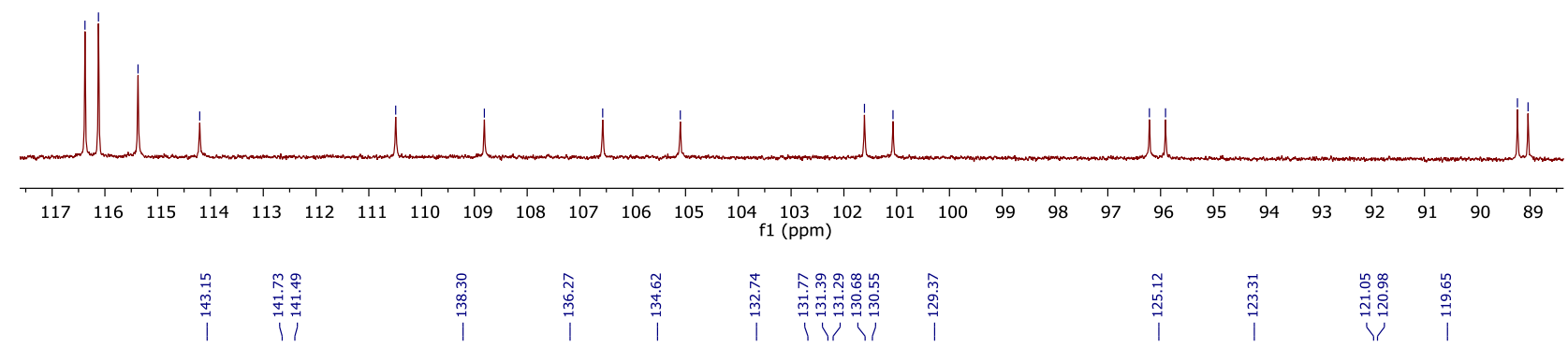

${ }^{13} \mathrm{C}$ NMR, $176 \mathrm{MHz}$, Methanol- $d_{4}$, Compound 7

$(118-146 \mathrm{ppm})$

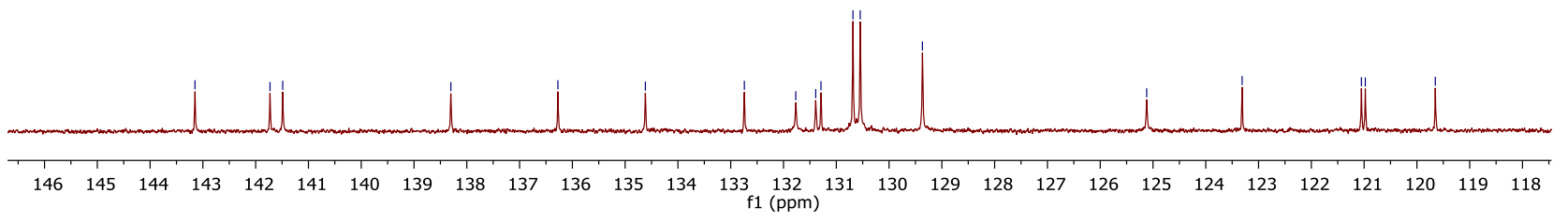

${ }^{13} \mathrm{C}$ NMR, $176 \mathrm{MHz}$, Methanol- $d_{4}$, Compound 7

$(147-175 \mathrm{ppm})$

草

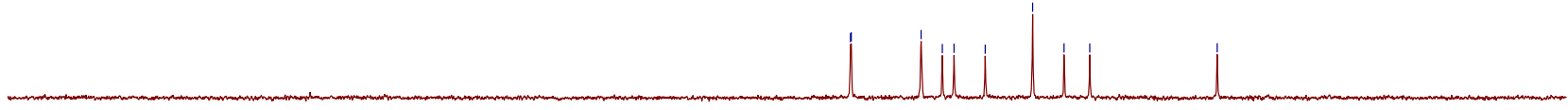

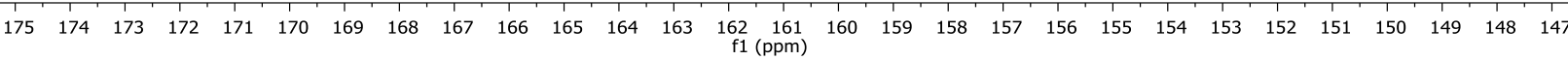


${ }^{1} \mathrm{H}$ NMR $\left(700 \mathrm{MHz}\right.$, Methanol- $\left.d_{4}\right) \delta 7.17\left(\mathrm{~d}, J=8.3 \mathrm{~Hz}, 2 \mathrm{H}, \mathrm{C}_{2 / 6 \mathrm{a}}-\underline{\mathrm{H}}\right), 7.07\left(\mathrm{~d}, J=8.3 \mathrm{~Hz}, 2 \mathrm{H}, \mathrm{C}_{2 / 6 \mathrm{~d}}-\underline{\mathrm{H}}\right), 7.02(\mathrm{~d}, J=8.2 \mathrm{~Hz}$, $\left.2 \mathrm{H}, \mathrm{C}_{2 / 6 \mathrm{~b}}-\underline{\mathrm{H}}\right), 6.73\left(\mathrm{~d}, J=8.4 \mathrm{~Hz}, 2 \mathrm{H}, \mathrm{C}_{3 / 5 \mathrm{~d}}-\underline{\mathrm{H}}\right), 6.72\left(\mathrm{~d}, J=8.4 \mathrm{~Hz}, 2 \mathrm{H}, \mathrm{C}_{3 / 5 \mathrm{a}}-\underline{\mathrm{H}}\right), 6.60\left(\mathrm{~d}, J=8.4 \mathrm{~Hz}, 2 \mathrm{H}, \mathrm{C}_{3 / 5 \mathrm{~b}}-\underline{\mathrm{H}}\right), 6.53(\mathrm{~d}$, $\left.J=8.5 \mathrm{~Hz}, 1 \mathrm{H}, \mathrm{C}_{5 \mathrm{c}}-\underline{\mathrm{H}}\right), 6.40\left(\mathrm{dd}, J=8.6,1.2 \mathrm{~Hz}, 1 \mathrm{H}, \mathrm{C}_{6 \mathrm{c}}-\underline{\mathrm{H}}\right), 6.23\left(\mathrm{~d}, J=2.3 \mathrm{~Hz}, 1 \mathrm{H}, \mathrm{C}_{12 \mathrm{~d}}-\underline{\mathrm{H}}\right), 6.20(\mathrm{~d}, J=2.3 \mathrm{~Hz}, 1 \mathrm{H}$, $\left.\mathrm{C}_{12 \mathrm{c}}-\underline{\mathrm{H}}\right), 6.11\left(\mathrm{~d}, J=2.3 \mathrm{~Hz}, 1 \mathrm{H}, \mathrm{C}_{14 \mathrm{~d}}-\underline{\mathrm{H}}\right), 6.06\left(\mathrm{~d}, J=2.3 \mathrm{~Hz}, 1 \mathrm{H}, \mathrm{C}_{14 \mathrm{c}}-\underline{\mathrm{H}}\right), 6.02\left(\mathrm{~d}, J=2.1 \mathrm{~Hz}, 1 \mathrm{H}, \mathrm{C}_{12 \mathrm{~b}}-\underline{\mathrm{H}}\right), 5.99(\mathrm{~d}, J=$ $\left.2.2 \mathrm{~Hz}, 1 \mathrm{H}, \mathrm{C}_{14 \mathrm{a}}-\underline{\mathrm{H}}\right), 5.95\left(\mathrm{~d}, J=1.2 \mathrm{~Hz}, 1 \mathrm{H}, \mathrm{C}_{2 \mathrm{c}}-\underline{\mathrm{H}}\right), 5.91\left(\mathrm{~d}, J=12.0 \mathrm{~Hz}, 1 \mathrm{H}, \mathrm{C}_{7 \mathrm{a}}-\underline{\mathrm{H}}\right), 5.90\left(\mathrm{~d}, J=2.4 \mathrm{~Hz}, 1 \mathrm{H}, \mathrm{C}_{12 \mathrm{a}}-\underline{\mathrm{H}}\right)$, $5.86\left(\mathrm{~d}, J=2.1 \mathrm{~Hz}, 1 \mathrm{H}, \mathrm{C}_{14 \mathrm{~b}}-\underline{\mathrm{H}}\right), 5.45\left(\mathrm{~d}, J=4.1 \mathrm{~Hz}, 1 \mathrm{H}, \mathrm{C}_{7 \mathrm{~b}}-\underline{\mathrm{H}}\right), 5.44\left(\mathrm{~d}, J=11.2 \mathrm{~Hz}, 1 \mathrm{H}, \mathrm{C}_{7 \mathrm{~d}}-\underline{\mathrm{H}}\right), 5.08(\mathrm{~d}, J=4.2 \mathrm{~Hz}, 1 \mathrm{H}$, $\left.\mathrm{C}_{8 \mathrm{~b}}-\underline{\mathrm{H}}\right), 4.74\left(\mathrm{t}, J=3.5 \mathrm{~Hz}, 1 \mathrm{H}, \mathrm{C}_{7 \mathrm{c}}-\underline{\mathrm{H}}\right), 4.03\left(\mathrm{~d}, J=11.9 \mathrm{~Hz}, 1 \mathrm{H}, \mathrm{C}_{8 \mathrm{a}}-\underline{\mathrm{H}}\right), 3.74\left(\mathrm{~d}, J=11.3 \mathrm{~Hz}, 1 \mathrm{H}, \mathrm{C}_{8 \mathrm{~d}}-\underline{\mathrm{H}}\right), 2.77(\mathrm{dd}, J=$ 17.1, $\left.2.7 \mathrm{~Hz}, 1 \mathrm{H}, \mathrm{C}_{8 \mathrm{c}}-\underline{\mathrm{H}}\right), 2.70\left(\mathrm{dd}, J=17.6,2.6 \mathrm{~Hz}, 1 \mathrm{H}, \mathrm{C}_{8 \mathrm{c}}-\underline{\mathrm{H}}\right)$.

${ }^{13} \mathrm{C}$ NMR $\left(126 \mathrm{MHz}\right.$, Methanol- $\left.d_{4}\right) \delta 160.1,159.9,158.7,158.47,158.43,158.35,157.5,156.9,156.6,156.5,155.8,152.2$, 143.5, 142.6, 141.6, 138.0, 136.3, 134.9, 131.7, 131.6, 131.4, 130.7, 130.6, 129.7, 129.3, 127.0, 124.3, 121.6, 119.7, 119.2, 116.1, 116.0, 115.4, 115.3, 109.8, 109.5, 106.0, 105.0, 101.2, 100.9, 96.2, 95.7, 89.3, 88.5, 49.5, 49.3, 41.8, 40.7, 36.2, 32.9.

HRMS (ESI) $m / z$ calculated for $\mathrm{C}_{56} \mathrm{H}_{43} \mathrm{O}_{12}{ }^{+}\left([\mathrm{M}+\mathrm{H}]^{+}\right)$907.2749, found 907.2741.

${ }^{1} \mathrm{H}$ NMR, $700 \mathrm{MHz}$, Methanol- $d_{4}$, Compound 26

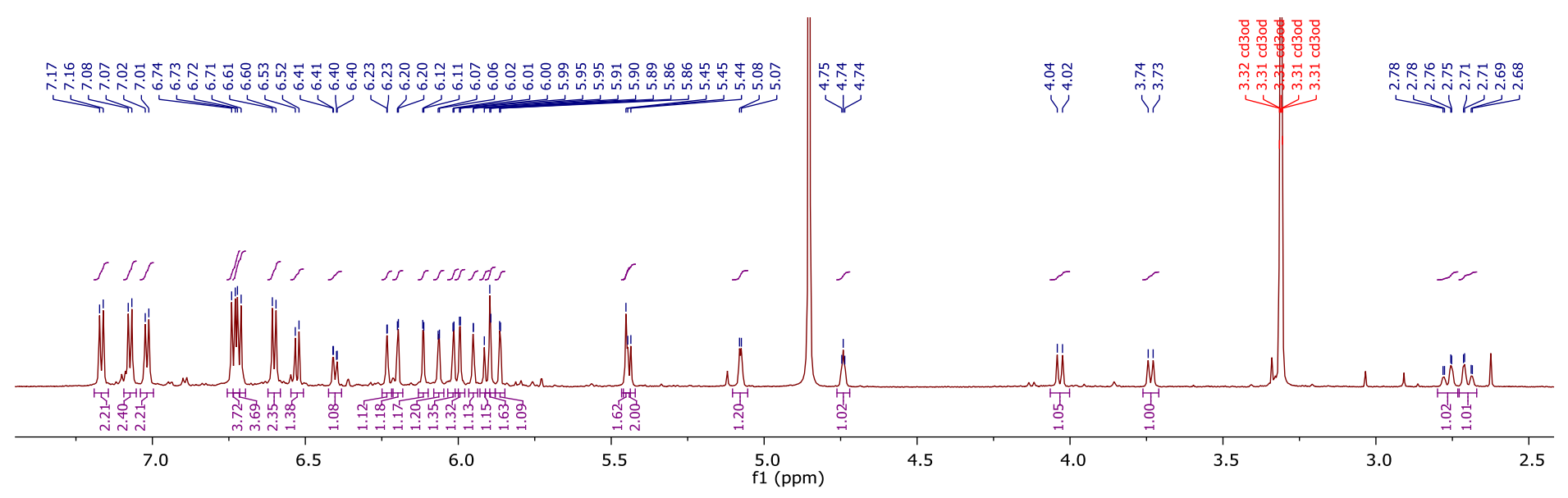

${ }^{13} \mathrm{C}$ NMR, $126 \mathrm{MHz}$, Methanol- $d_{4}$, Compound 26

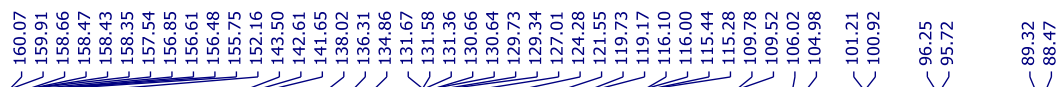

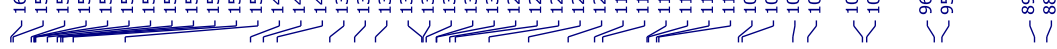

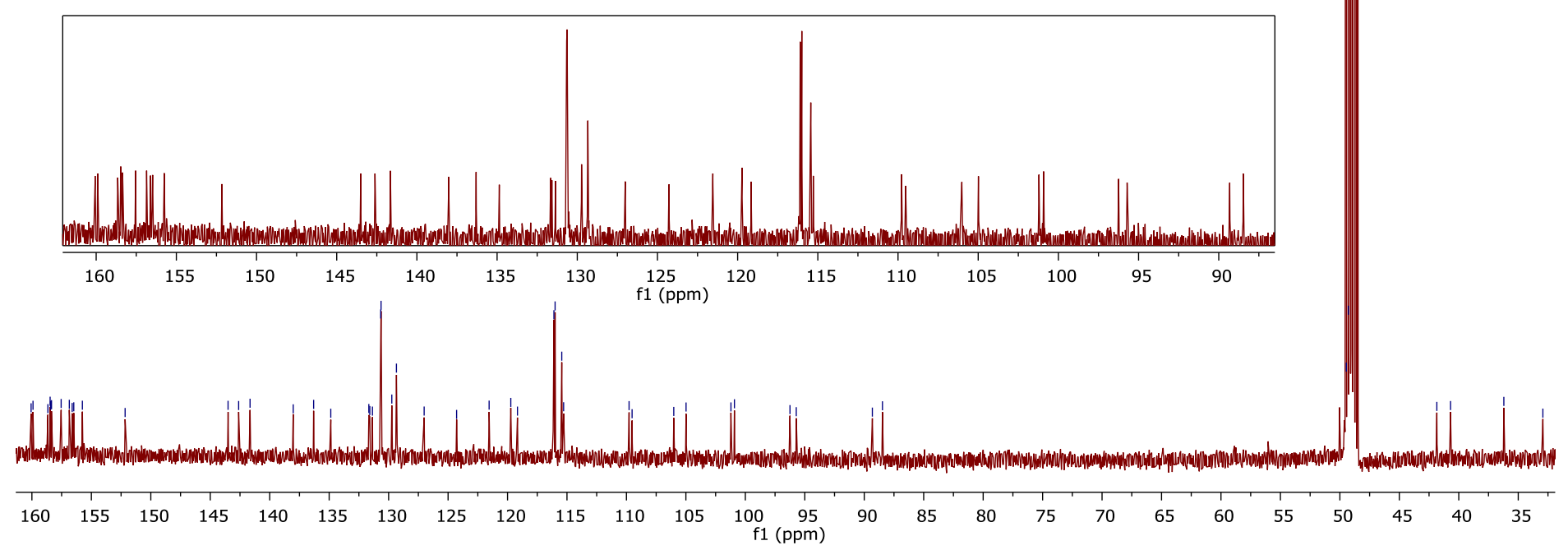


COSY, 700 MHz, Methanol- $d_{4}$, Compound 26

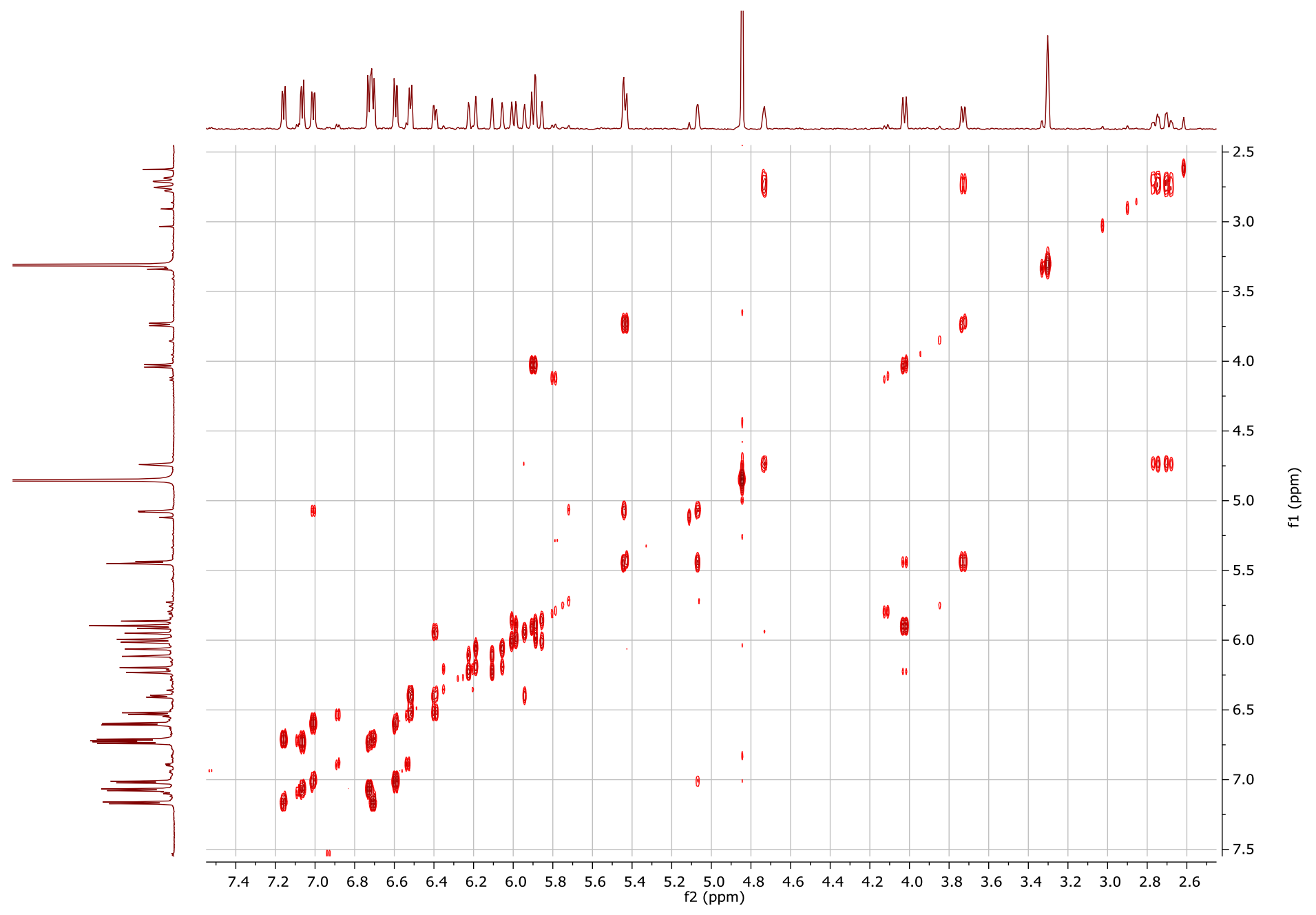


Table S4. Comparison of literature and observed ${ }^{1} \mathrm{H}$ NMR data for vitisin D (7).

\begin{tabular}{|c|c|c|c|}
\hline & \multicolumn{2}{|c|}{ Literature } & Observed \\
\hline Assignment & $300 \mathrm{MHz}, \mathrm{CDCl}: \mathrm{CD}_{3} \mathrm{OD}(7: 3)^{8}$ & $600 \mathrm{MHz}, \mathrm{CD}_{3} \mathrm{OD}^{9}$ & $700 \mathrm{MHz}, \mathrm{CD}_{3} \mathrm{OD}$ \\
\hline $8 \mathrm{c}$ & $2.98(1 \mathrm{H} \mathrm{br} \mathrm{d}, J=16.8 \mathrm{~Hz})$ & $2.93(\mathrm{br} \mathrm{d}, J=16.6 \mathrm{~Hz})$ & $2.90(\mathrm{dd}, J=17.8,3.7 \mathrm{~Hz}, 1 \mathrm{H})$ \\
\hline $8 c$ & $3.15(1 \mathrm{H}$, br d, $J=16.8 \mathrm{~Hz})$ & $3.14(\mathrm{br} \mathrm{d}, J=16.6 \mathrm{~Hz})$ & $3.11(\mathrm{dd}, J=18.0,3.8 \mathrm{~Hz}, 1 \mathrm{H})$ \\
\hline $8 \mathrm{~d}$ & $3.90(1 \mathrm{H}, \mathrm{d}, J=11.0 \mathrm{~Hz})$ & $3.39(\mathrm{~d}, J=11.1 \mathrm{~Hz})^{*}$ & $3.91(\mathrm{~d}, J=11.2 \mathrm{~Hz}, 1 \mathrm{H})$ \\
\hline $8 \mathrm{a}$ & $4.05(1 \mathrm{H}, \mathrm{d}, J=11.6 \mathrm{~Hz})$ & $4.01(\mathrm{~d}, J=11.9 \mathrm{~Hz})$ & $3.98(\mathrm{~d}, J=11.9 \mathrm{~Hz}, 1 \mathrm{H})$ \\
\hline $7 \mathrm{c}$ & $4.70(1 \mathrm{H}$, br t,$J=4.5 \mathrm{~Hz})$ & $4.66(\mathrm{br} \mathrm{t}, J=4.5 \mathrm{~Hz})$ & $4.68(\mathrm{t}, J=4.0 \mathrm{~Hz}, 1 \mathrm{H})$ \\
\hline $8 \mathrm{~b}$ & $5.26(1 \mathrm{H}, \mathrm{d}, J=3.7 \mathrm{~Hz})$ & $5.27(\mathrm{~d}, J=3.7 \mathrm{~Hz})$ & $5.24(\mathrm{~d}, J=4.3 \mathrm{~Hz}, 1 \mathrm{H})$ \\
\hline $7 b$ & $5.49(1 \mathrm{H}, \mathrm{d}, J=3.7 \mathrm{~Hz})$ & $5.59(\mathrm{~d}, J=3.7 \mathrm{~Hz})$ & $5.56(\mathrm{~d}, J=4.2 \mathrm{~Hz}, 1 \mathrm{H})$ \\
\hline $7 d$ & $5.63(1 \mathrm{H}, \mathrm{d}, J=11.0 \mathrm{~Hz})$ & $5.58(\mathrm{~d}, J=11.0 \mathrm{~Hz})$ & $5.55(\mathrm{~d}, J=11.1 \mathrm{~Hz}, 1 \mathrm{H})$ \\
\hline $7 \mathrm{a}$ & $5.70(1 \mathrm{H}, \mathrm{d}, J=11.6 \mathrm{~Hz})$ & $5.75(\mathrm{~d}, J=11.6 \mathrm{~Hz})$ & $5.73(\mathrm{~d}, J=11.9 \mathrm{~Hz}, 1 \mathrm{H})$ \\
\hline $14 b$ & $5.79(1 \mathrm{H}$, br s $)$ & $5.77($ br s $)$ & $5.83(\mathrm{~s}, 1 \mathrm{H})$ \\
\hline $12 \mathrm{a}$ & $5.82(1 \mathrm{H}$, br s $)$ & $5.86($ br s) & $5.84(\mathrm{~d}, J=2.9 \mathrm{~Hz}, 1 \mathrm{H})$ \\
\hline $14 \mathrm{a}$ & $5.89(1 \mathrm{H}$, br s $)$ & 5.87 (br s) & $5.87(\mathrm{~s}, 1 \mathrm{H})$ \\
\hline $2 c$ & $5.92(1 \mathrm{H}$, br s $)$ & 5.90 (br s) & $5.96(\mathrm{~s}, 1 \mathrm{H})$ \\
\hline $14 \mathrm{~d}$ & $5.98(1 \mathrm{H}$, br s $)$ & 5.99 (br s) & $5.98(\mathrm{~d}, J=2.1 \mathrm{~Hz}, 1 \mathrm{H})$ \\
\hline $12 b$ & $6.02(1 \mathrm{H}$, br s $)$ & 6.02 (br s) & $6.00(\mathrm{~s}, 1 \mathrm{H})$ \\
\hline $12 \mathrm{c}$ & $6.07(1 \mathrm{H}$, br s) & 6.06 (br s) & $6.02(\mathrm{~s}, 1 \mathrm{H})$ \\
\hline $14 \mathrm{c}$ & $6.07(1 \mathrm{H}$, br s $)$ & 6.06 (br s) & $6.03(\mathrm{~s}, 1 \mathrm{H})$ \\
\hline $12 \mathrm{~d}$ & $6.15(1 \mathrm{H}$, br s $)$ & $6.19(\mathrm{br} \mathrm{s})$ & $6.15(\mathrm{~d}, J=2.2 \mathrm{~Hz}, 1 \mathrm{H})$ \\
\hline $5 c$ & $6.52(1 \mathrm{H}, \mathrm{d}, J=7.4 \mathrm{~Hz})$ & $6.52(\mathrm{~d}, J=7.4 \mathrm{~Hz})$ & $6.49(\mathrm{~d}, J=8.5 \mathrm{~Hz}, 1 \mathrm{H})$ \\
\hline $6 c$ & $6.57(1 \mathrm{H}$, br d, $J=7.4 \mathrm{~Hz})$ & $6.53(\mathrm{brd}, J=7.4 \mathrm{~Hz})$ & $6.51(\mathrm{~d}, J=8.4 \mathrm{~Hz}, 1 \mathrm{H})$ \\
\hline $3 / 5 b$ & $6.60(2 \mathrm{H}, \mathrm{d}, J=8.4 \mathrm{~Hz})$ & $6.62(\mathrm{~d}, J=8.4 \mathrm{~Hz})$ & $6.57(\mathrm{~d}, J=8.3 \mathrm{~Hz}, 2 \mathrm{H})$ \\
\hline $3 / 5 \mathrm{a}$ & $6.73(2 \mathrm{H}, \mathrm{d}, J=8.4 \mathrm{~Hz})$ & $6.74(\mathrm{~d}, J=8.4 \mathrm{~Hz})$ & $6.70(\mathrm{~d}, J=8.2 \mathrm{~Hz}, 2 \mathrm{H})$ \\
\hline $3 / 5 \mathrm{~d}$ & $6.85(2 \mathrm{H}, \mathrm{d}, J=8.4 \mathrm{~Hz})$ & $6.86(\mathrm{~d}, J=8.4 \mathrm{~Hz})$ & $6.83(\mathrm{~d}, J=8.1 \mathrm{~Hz}, 2 \mathrm{H})$ \\
\hline $2 / 6 b$ & $6.98(2 \mathrm{H}, \mathrm{d}, J=8.4 \mathrm{~Hz})$ & $6.97(\mathrm{~d}, J=8.4 \mathrm{~Hz})$ & $6.94(\mathrm{~d}, J=8.2 \mathrm{~Hz}, 2 \mathrm{H})$ \\
\hline $2 / 6 \mathrm{~d}$ & $7.09(2 \mathrm{H}, \mathrm{d}, J=8.4 \mathrm{~Hz})$ & $7.09(\mathrm{~d}, J=8.4 \mathrm{~Hz})$ & $7.06(\mathrm{~d}, J=8.2 \mathrm{~Hz}, 2 \mathrm{H})$ \\
\hline $2 / 6 \mathrm{a}$ & $7.15(2 \mathrm{H}, \mathrm{d}, J=8.4 \mathrm{~Hz})$ & $7.14(\mathrm{~d}, J=8.4 \mathrm{~Hz})$ & $7.11(\mathrm{~d}, J=8.2 \mathrm{~Hz}, 2 \mathrm{H})$ \\
\hline
\end{tabular}

*We believe that this signal was incorrectly tabulated in ref. 9 and should instead be $3.93 \mathrm{ppm}$. 
Table S5. Comparison of literature and observed ${ }^{13} \mathrm{C}$ NMR data for vitisin D (7).

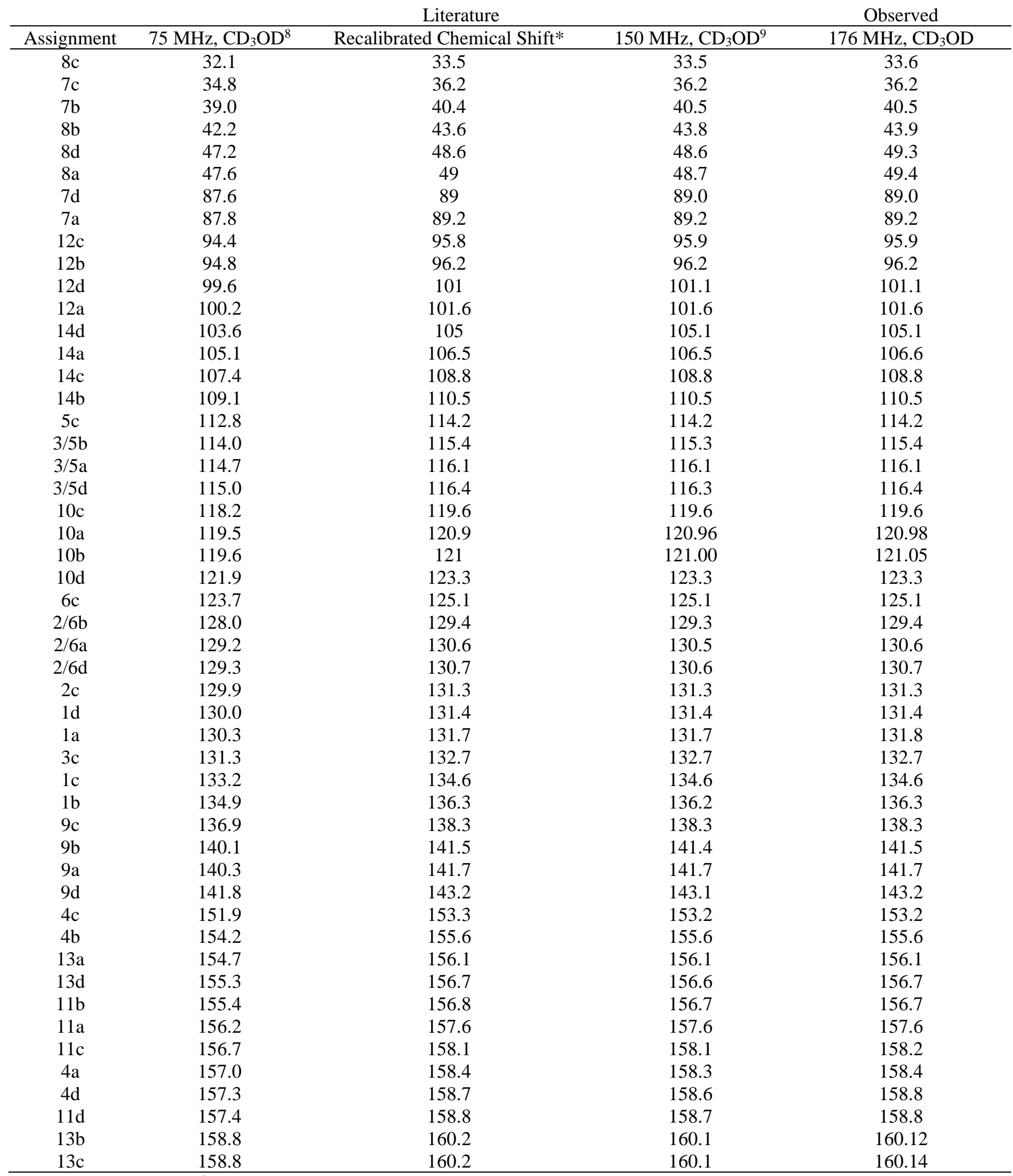

*We noticed that the ${ }^{13} \mathrm{C}$ NMR data from ref. 8 was consistently $\sim 1.4$ ppm lower than observed, perhaps due to incorrect referencing of the solvent signal. After recalibrating the data from ref. 8 by adding $1.4 \mathrm{ppm}$ to each signal, it is consistent with the observed data as well as ref. 9. 

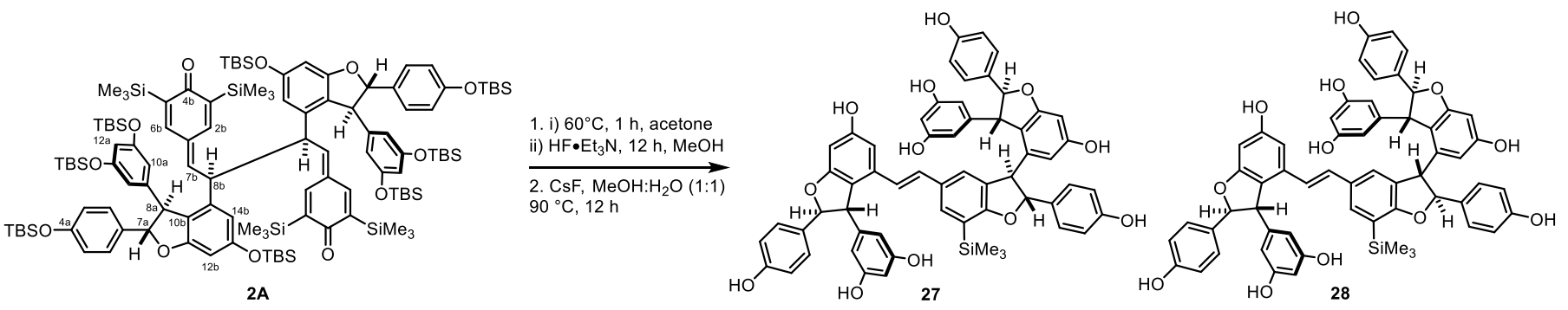

27 - 5-((2S,3S)-4-((E)-2-((2R,2'S,3R,3'S)-3'-(3,5-dihydroxyphenyl)-6'-hydroxy-2,2'-bis(4-hydroxyphenyl)-7(trimethylsilyl)-2,2',3,3'-tetrahydro-[3,4'-bibenzofuran]-5-yl)vinyl)-6-hydroxy-2-(4-hydroxyphenyl)-2,3dihydrobenzofuran-3-yl)benzene-1,3-diol

Quinone-methide dimer 2A (200 mg, $0.095 \mathrm{mmol}$ ) was added to a flame-dried reaction vial charged with a stir bar and dissolved in acetone ( $3.0 \mathrm{~mL}$, HPLC grade). The reaction solution was sparged with a balloon of Ar for 5 minutes and then sealed under argon with parafilm. The reaction was heated to $60{ }^{\circ} \mathrm{C}$ for 1 hour, and upon cooling to ambient temperature, a solution of HF-triethylamine was added $(0.93 \mathrm{~mL}, 5.68 \mathrm{mmol})$ followed by MeOH (3.0 mL, HPLC grade). The reaction was sealed under an argon atmosphere and allowed to stir at ambient temperature. After 16 hours, the stir bar was removed, and the reaction mixture was added to a separatory funnel containing sat. aq. sodium bicarbonate $(\sim 50 \mathrm{~mL})$. The aqueous layer was extracted with portion of EtOAc $(3 \times 25 \mathrm{~mL})$, and the combined organic layers were washed with brine, dried over $\mathrm{MgSO}_{4}$, and concentrated under reduced pressure. The crude material was dissolved in $\mathrm{MeOH} / \mathrm{H}_{2} \mathrm{O}(1: 1,3.0 \mathrm{~mL})$, and to the reaction mixture was added $\mathrm{CsF}(143 \mathrm{mg}, 0.944 \mathrm{mmol})$. The reaction was sealed under nitrogen and heated at $90{ }^{\circ} \mathrm{C}$ for 12 hours. Upon cooling to room temperature, the reaction mixture was added to separatory funnel containing saturated aqueous $\mathrm{NH}_{4} \mathrm{Cl}$, and the aqueous layer was extracted with portions of EtOAc $(3 \times 25 \mathrm{~mL})$. The combined organic layers were washed with brine, dried over $\mathrm{MgSO}_{4}$, and concentrated under reduced pressure. The crude material was purified by flash column chromatography (8\% to $40 \%$ in 5 step increments, 2 column volumes per step, Nonpolar solvent $-\mathrm{CH}_{2} \mathrm{Cl}_{2}$, Polar solvent - 3:1 Acetone/MeOH) to afford the $\mathbf{2 7 / 2 8}$ mixture (67.5 mg, $73 \%$ yield).

${ }^{1} \mathrm{H}$ NMR $\left(700 \mathrm{MHz}\right.$, Acetone- $\left.d_{6}\right) \delta 8.25(\mathrm{~s}, 20 \mathrm{H}), 7.26(\mathrm{~d}, J=8.3 \mathrm{~Hz}, 4 \mathrm{H}), 7.20(\mathrm{td}, J=6.7,3.1 \mathrm{~Hz}, 8 \mathrm{H}), 6.94-6.90(\mathrm{~m}$, $4 \mathrm{H}), 6.89(\mathrm{~d}, J=8.4 \mathrm{~Hz}, 2 \mathrm{H}), 6.86-6.80(\mathrm{~m}, 7 \mathrm{H}), 6.78(\mathrm{~d}, J=17.4 \mathrm{~Hz}, 2 \mathrm{H}), 6.69(\mathrm{~d}, J=2.0 \mathrm{~Hz}, 1 \mathrm{H}), 6.67(\mathrm{~d}, J=2.0 \mathrm{~Hz}$, $1 \mathrm{H}), 6.63(\mathrm{~d}, J=6.4 \mathrm{~Hz}, 2 \mathrm{H}), 6.62-6.57(\mathrm{~m}, 10 \mathrm{H}), 6.31(\mathrm{~d}, J=2.7 \mathrm{~Hz}, 3 \mathrm{H}), 6.30(\mathrm{~d}, J=2.0 \mathrm{~Hz}, 1 \mathrm{H}), 6.25(\mathrm{q}, J=2.3 \mathrm{~Hz}$, $2 \mathrm{H}), 6.24-6.21(\mathrm{~m}, 2 \mathrm{H}), 6.20(\mathrm{~d}, J=2.1 \mathrm{~Hz}, 1 \mathrm{H}), 6.18(\mathrm{~d}, J=2.2 \mathrm{~Hz}, 2 \mathrm{H}), 6.17(\mathrm{~d}, J=2.1 \mathrm{~Hz}, 1 \mathrm{H}), 6.16(\mathrm{~d}, J=2.2 \mathrm{~Hz}$, 2H), $6.13(\mathrm{dd}, J=5.0,2.2 \mathrm{~Hz}, 4 \mathrm{H}), 5.55(\mathrm{~d}, J=4.7 \mathrm{~Hz}, 1 \mathrm{H}), 5.54(\mathrm{~d}, J=4.2 \mathrm{~Hz}, 1 \mathrm{H}), 5.41(\mathrm{~d}, J=4.4 \mathrm{~Hz}, 1 \mathrm{H}), 5.40(\mathrm{~d}, J=$ $4.2 \mathrm{~Hz}, 1 \mathrm{H}), 5.37(\mathrm{~d}, J=4.8 \mathrm{~Hz}, 1 \mathrm{H}), 5.36(\mathrm{~s}, 1 \mathrm{H}), 4.56(\mathrm{~d}, J=4.4 \mathrm{~Hz}, 1 \mathrm{H}), 4.53(\mathrm{~d}, J=4.4 \mathrm{~Hz}, 1 \mathrm{H}), 4.45(\mathrm{~s}, 1 \mathrm{H}), 4.44(\mathrm{~d}$, $J=5.6 \mathrm{~Hz}, 1 \mathrm{H}), 4.25(\mathrm{~d}, J=4.6 \mathrm{~Hz}, 1 \mathrm{H}), 4.24(\mathrm{~d}, J=4.2 \mathrm{~Hz}, 1 \mathrm{H}), 0.29-0.28(\mathrm{~m}, 18 \mathrm{H})$.

${ }^{13} \mathrm{C}$ NMR $\left(176 \mathrm{MHz}\right.$, Acetone- $\left.d_{6}\right) \delta 164.92,164.86,162.71,162.69,162.47,162.45,160.3,160.2,159.9,159.8,159.5$, 158.3, 158.1, 158.0, 157.7, 147.63, 147.55, 147.4, 147.3, 142.8, 142.6, 136.63, 136.59, 134.4, 134.3, 133.89, 133.87, 133.1, $132.9,132.3,131.7,131.31,131.29,131.2,131.1,130.8,130.7,130.2,129.2,128.9,128.2,128.1,128.01,127.95,127.3$, $127.2,126.9,126.4,123.7,123.6,120.9,120.8,119.71,119.68,119.65,119.64,116.43,116.41,116.2,115.9,107.0,104.7$, 104.6, 102.5, 102.3, 96.8, 96.6, 94.2, 94.1, 90.84, 90.76, 57.3, 57.2, 56.9, 56.7, 52.5, 52.3, - $0.82,-0.84$.

IR (Neat): 3439 (br), 2923, 1617, 1586, 1525, 1444, 1328, 1269, 1158, 1135, $1015 \mathrm{~cm}^{-1}$.

HRMS (ESI) $m / z$ calculated for $\mathrm{C}_{59} \mathrm{H}_{51} \mathrm{O}_{12} \mathrm{Si}^{+}\left([\mathrm{M}+\mathrm{H}]^{+}\right)$979.3144, found 979.3130. 
${ }^{1} \mathrm{H}$ NMR, $700 \mathrm{MHz}$, Acetone-d6, Compounds 27/28

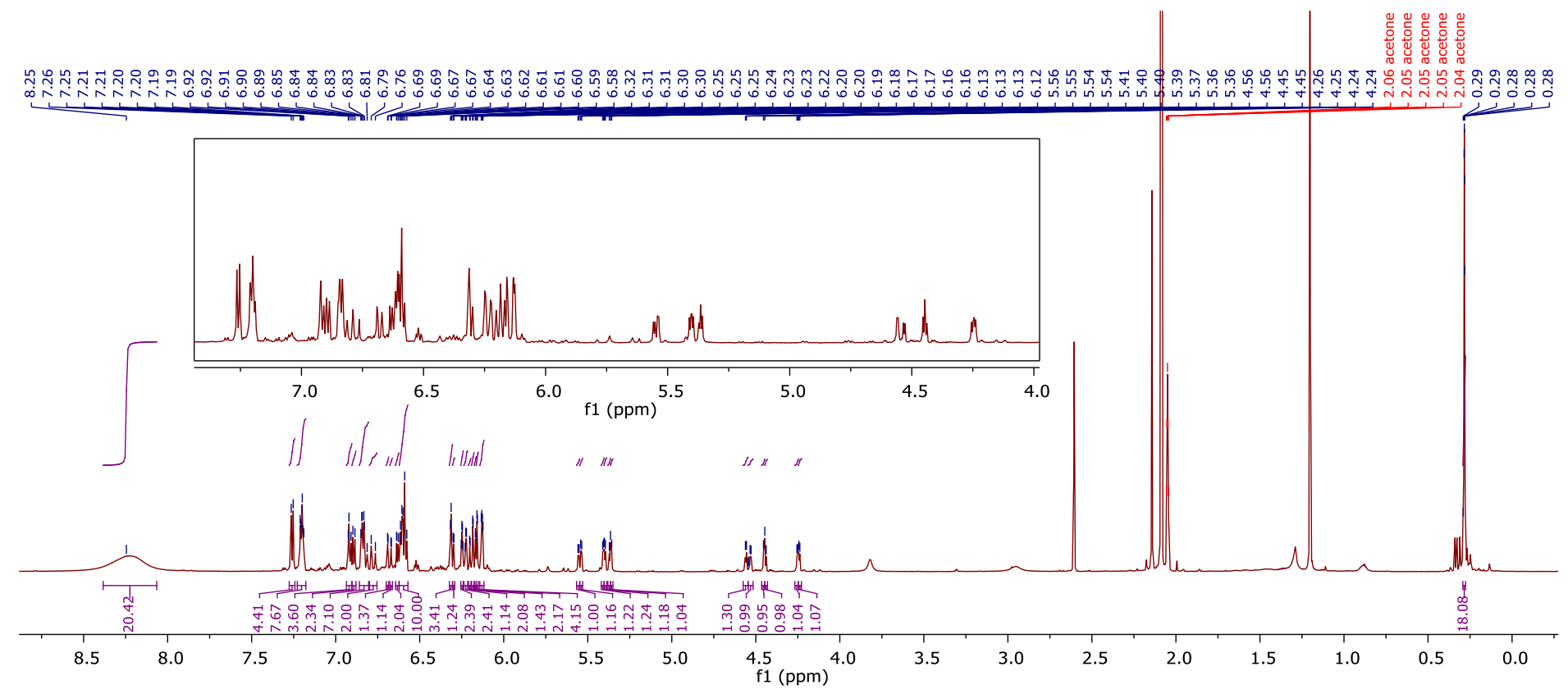

${ }^{13} \mathrm{C}$ NMR, $176 \mathrm{MHz}$, Acetone-d6, Compounds 27/28
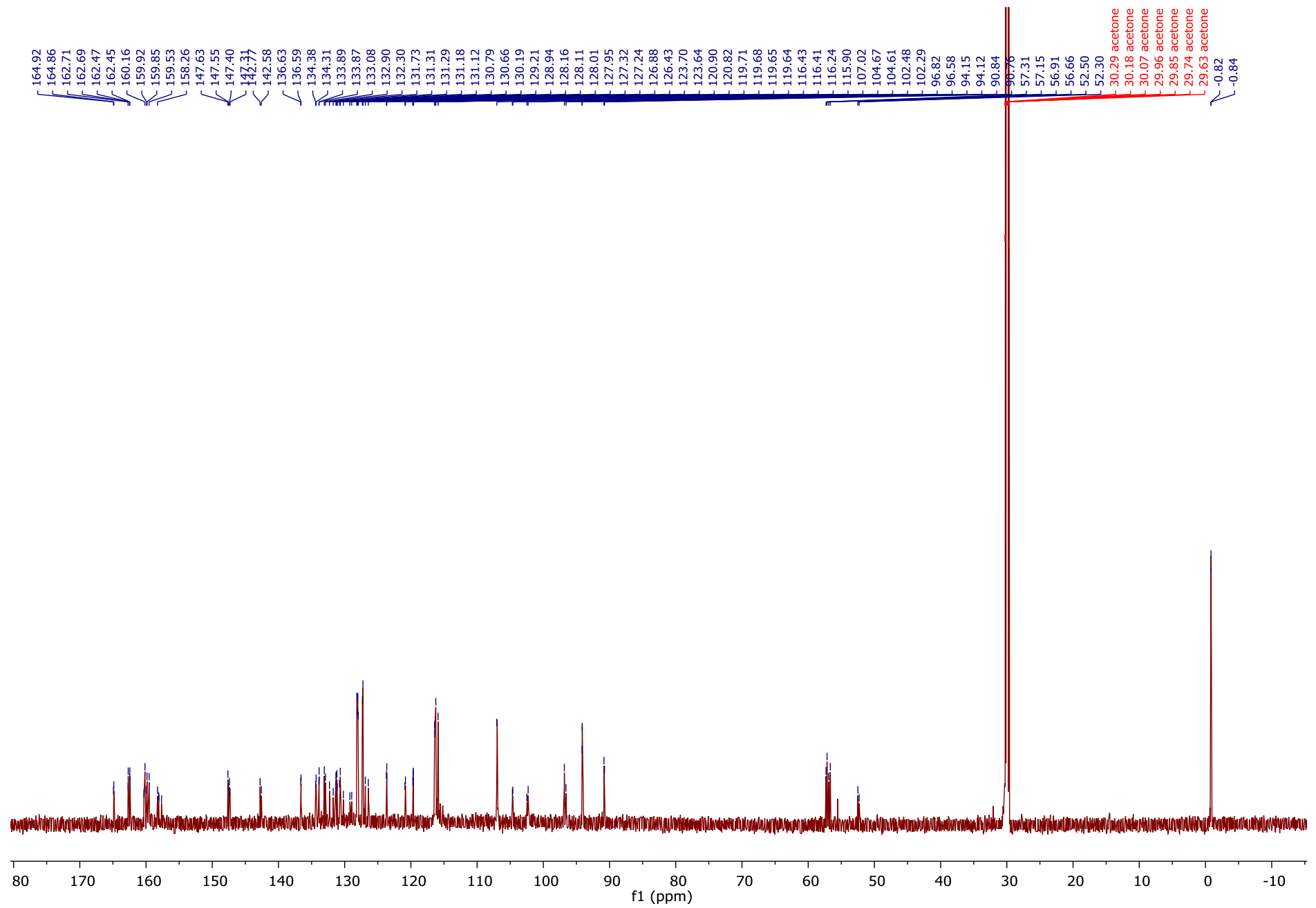
Table S6. Selected examples of C5c-desilylation efforts
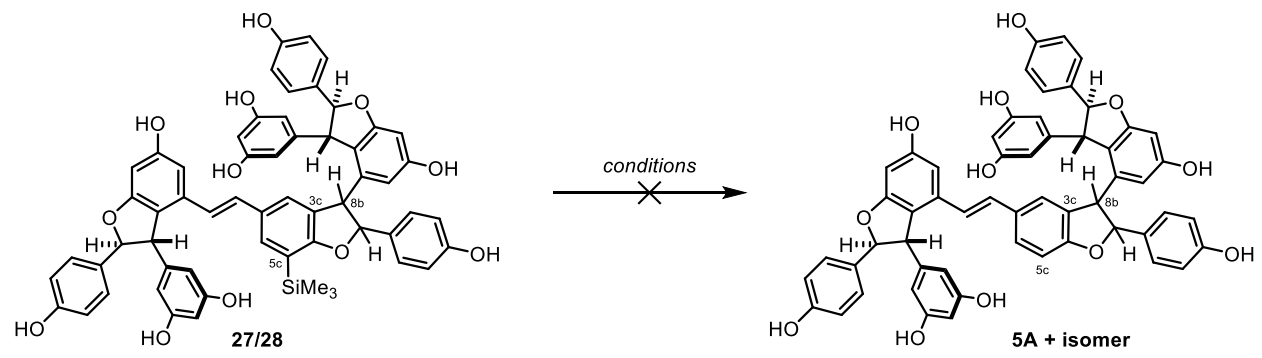

\begin{tabular}{|c|c|c|}
\hline Entry & Conditions & Result \\
\hline 1 & TBAF (1 M in THF), DMF, $90{ }^{\circ} \mathrm{C}, 16 \mathrm{~h}$ & Decomposition of DHB rings \\
\hline 2 & $\mathrm{KHF}_{2}, \mathrm{DMF}, 90^{\circ} \mathrm{C}, 16 \mathrm{~h}$ & No conversion \\
\hline 3 & HF-Et 3 N, DMF, $90^{\circ} \mathrm{C}, 16 \mathrm{~h}$ & No conversion \\
\hline 4 & TMAF, DMF, rt, $16 \mathrm{~h}$ & No conversion \\
\hline 5 & TMAF, DMF, $60^{\circ} \mathrm{C}, 16 \mathrm{~h}$ & No conversion \\
\hline 6 & TMAF, DMF, $90^{\circ} \mathrm{C}, 16 \mathrm{~h}$ & Full decomp. Of C3c-C8b DHB, $\sim 20 \%$ conversion to vitisin A \\
\hline 7 & DAST (1M in MeCN), MeCN, rt, $16 \mathrm{~h}$ & No conversion \\
\hline 8 & DAST $(1 \mathrm{M}$ in $\mathrm{MeCN}), \mathrm{DMF}, 0^{\circ} \mathrm{C}$ to $\mathrm{rt}, 3 \mathrm{~h}$ & Decomposition \\
\hline 9 & $\mathrm{CsF}, \mathrm{MeOH} / \mathrm{H}_{2} \mathrm{O}(1: 1), 120^{\circ} \mathrm{C}, 16 \mathrm{~h}$ & Decomposition \\
\hline 10 & $\mathrm{CsF}, \mathrm{MeOH} / \mathrm{H}_{2} \mathrm{O}(1: 1), 90^{\circ} \mathrm{C}, 6 \mathrm{~h}$ & No conversion \\
\hline 11 & $\mathrm{CsF}, \mathrm{MeOH} / \mathrm{H}_{2} \mathrm{O}(1: 1), 100{ }^{\circ} \mathrm{C}, 12 \mathrm{~h}$ & No conversion \\
\hline 12 & $\mathrm{CsF}, \mathrm{MeOH} / \mathrm{H}_{2} \mathrm{O}(1: 1), 110^{\circ} \mathrm{C}, 6 \mathrm{~h}$ & No conversion \\
\hline 13 & $\mathrm{CsF}, \mathrm{MeOH} / \mathrm{H}_{2} \mathrm{O}(1: 1), 120^{\circ} \mathrm{C}, 6 \mathrm{~h}$ & Decomposition \\
\hline 14 & TFA, $\mathrm{CH}_{2} \mathrm{Cl}_{2} / \mathrm{MeNO}_{2}(1: 1), \mathrm{rt}, 2 \mathrm{~h}$ & vitisin A (25\%) and vitisin D (7\%) \\
\hline
\end{tabular}




\section{Computational Data.}

Calculations were carried out at the B3LYP-D3 level of theory with the 6-311G(d,p) basis set as implemented in the Gaussian 16 suite of programs.

Table S7. Enthalpies and free energies (in $\mathrm{kcal} / \mathrm{mol}$ ) for the dimerization of resveratrol radicals to dimers.

\begin{tabular}{c|cc|c|cc|c|cc}
$\mathbf{R}=\mathbf{H}$ & $\boldsymbol{\Delta H}_{\mathbf{r}}$ & $\boldsymbol{\Delta} \mathbf{G}_{\mathbf{r}}$ & $\mathbf{R}={ }^{t} \mathbf{B u}$ & $\boldsymbol{\Delta} \mathbf{H}_{\mathbf{r}}$ & $\boldsymbol{\Delta} \mathbf{G}_{\mathbf{r}}$ & $\mathbf{R}=\mathbf{S i M e}_{\mathbf{3}}$ & $\boldsymbol{\Delta} \mathbf{H}_{\mathbf{r}}$ & $\boldsymbol{\Delta} \mathbf{G}_{\mathbf{r}}$ \\
\hline $\mathbf{9 C}$ & 0.0 & 0.0 & $\mathbf{9 B}$ & 0.0 & 0.0 & $\mathbf{9 A}$ & 0.0 & 0.0 \\
$\mathbf{8 C}$ & -16.2 & -1.7 & $\mathbf{8 B}$ & -23.4 & -5.1 & $\mathbf{8 A}$ & -25.3 & -6.1 \\
$\mathbf{1 0 C}$ & -12.6 & 1.1 & $\mathbf{1 0 B}$ & -9.6 & 7.5 & $\mathbf{1 0 A}$ & -17.9 & -1.9
\end{tabular}

Table S8. Relative enthalpies and free energies (in $\mathrm{kcal} / \mathrm{mol}$ ) for $\mathbf{R}={ }^{t} \mathrm{Bu}$ and $\mathrm{TMS}$.

\begin{tabular}{c|cc|c|cc}
$\mathbf{R}={ }^{\boldsymbol{t}} \mathbf{B u}$ & $\boldsymbol{\Delta} \boldsymbol{\Delta} \mathbf{H}_{\mathbf{r}}$ & $\boldsymbol{\Delta} \boldsymbol{\Delta} \mathbf{G}_{\mathbf{r}}$ & $\mathbf{R}=\mathbf{S i M e}_{\mathbf{3}}$ & $\boldsymbol{\Delta} \boldsymbol{\Delta} \mathbf{H}_{\mathbf{r}}$ & $\boldsymbol{\Delta} \boldsymbol{\Delta} \mathbf{G}_{\mathbf{r}}$ \\
\hline 8B & 0.0 & 0.0 & $8 \mathrm{~A}$ & -1.9 & -1.1 \\
10B & 13.9 & 12.6 & $10 \mathrm{~A}$ & 5.5 & 3.1
\end{tabular}

Optimized Structures and B3LYP-D3/6-311G(d,p) Energies

resveratrol radical<smiles>Oc1cc(O)cc(/C=C/c2ccc(Cl)cc2)c1</smiles>

Enthalpy $=\quad-765.728708 \quad$ Free Energy $=\quad-765.788163$

02

$\begin{array}{lrrr}\mathrm{C} & 4.11301400 & -1.28211300 & 0.00000700 \\ \mathrm{C} & 2.76489500 & -1.09696700 & 0.00000700 \\ \mathrm{C} & 2.19113900 & 0.21564700 & -0.00000100 \\ \mathrm{C} & 3.08432200 & 1.33160200 & -0.00000600 \\ \mathrm{C} & 4.43837800 & 1.17148200 & -0.00000700 \\ \mathrm{C} & 5.04462100 & -0.15566200 & -0.00000700 \\ \mathrm{O} & 6.27368500 & -0.31983900 & 0.00000300 \\ \mathrm{C} & 0.78165400 & 0.46213200 & -0.00000100 \\ \mathrm{C} & -0.21287900 & -0.47040700 & 0.00000300 \\ \mathrm{C} & -1.64492200 & -0.21869100 & 0.00000200 \\ \mathrm{C} & -2.50670500 & -1.32505300 & 0.00000200 \\ \mathrm{C} & -3.88976200 & -1.14198600 & 0.00000000 \\ \mathrm{C} & -4.43262400 & 0.13997900 & -0.00000100 \\ \mathrm{C} & -3.57506100 & 1.24592300 & -0.00000100 \\ \mathrm{C} & -2.19644600 & 1.07911000 & 0.00000000 \\ \mathrm{O} & -4.17269700 & 2.47182900 & -0.00000300 \\ \mathrm{O} & -4.66234800 & -2.26464600 & 0.00000000 \\ \mathrm{H} & 4.55384800 & -2.27215400 & 0.00001400\end{array}$




$\begin{array}{rrrr}\mathrm{H} & 2.11164200 & -1.96156700 & 0.00001400 \\ \mathrm{H} & 2.65749300 & 2.33008200 & -0.00000900 \\ \mathrm{H} & 5.11686100 & 2.01638400 & -0.00001000 \\ \mathrm{H} & 0.50273100 & 1.51207600 & -0.00000600 \\ \mathrm{H} & 0.05991000 & -1.52090200 & 0.00000700 \\ \mathrm{H} & -2.11773600 & -2.33548600 & 0.00000300 \\ \mathrm{H} & -5.50380100 & 0.30730200 & -0.00000300 \\ \mathrm{H} & -1.55710700 & 1.95421000 & 0.00000100 \\ \mathrm{H} & -3.49801500 & 3.15848500 & -0.00000300 \\ \mathrm{H} & -5.59268300 & -2.01717000 & -0.00000100\end{array}$

resveratrol dimer (C8-C8', meso)

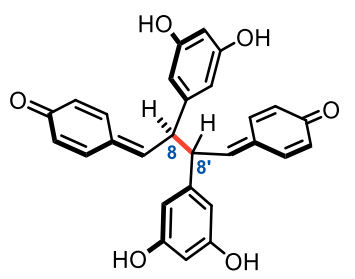

$\begin{array}{lrrr}\text { Enthalpy }= & -1531.483309 & \text { Free Energy= } \\ \text { O 1 } & & & \\ \mathrm{C} & -2.49402300 & 1.58114400 & 0.34129800 \\ \mathrm{C} & -1.19547500 & 1.35151600 & 0.65930800 \\ \mathrm{C} & -0.12294700 & 0.76074100 & -0.20442800 \\ \mathrm{C} & 0.12294900 & -0.76074700 & 0.20443200 \\ \mathrm{C} & 1.19547300 & -1.35152200 & -0.65930900 \\ \mathrm{C} & 2.49402400 & -1.58114500 & -0.34130700 \\ \mathrm{H} & -0.44450400 & 0.75371100 & -1.24646600 \\ \mathrm{C} & -3.07629300 & 1.31140400 & -0.96796100 \\ \mathrm{C} & -4.38875800 & 1.48675100 & -1.20769200 \\ \mathrm{C} & -5.31794700 & 1.96890000 & -0.16795900 \\ \mathrm{C} & -4.71037400 & 2.27650200 & 1.14043900 \\ \mathrm{C} & -3.39650600 & 2.09723600 & 1.36654600 \\ \mathrm{C} & 3.39650000 & -2.09724400 & -1.36655700 \\ \mathrm{C} & 4.71037000 & -2.27650800 & -1.14045700 \\ \mathrm{C} & 5.31795200 & -1.96889200 & 0.16793300 \\ \mathrm{C} & 4.38876800 & -1.48674300 & 1.20767100 \\ \mathrm{C} & 3.07630200 & -1.31139800 & 0.96794800 \\ \mathrm{O} & -6.51662700 & 2.10697000 & -0.38075800 \\ \mathrm{O} & 6.51663100 & -2.10697500 & 0.38072900 \\ \mathrm{C} & 1.17745500 & 1.54405500 & -0.11465300 \\ \mathrm{C} & 1.80419300 & 1.73964200 & 1.11319200 \\ \mathrm{C} & 3.05620500 & 2.35472400 & 1.16531000 \\ \mathrm{C} & 3.67622500 & 2.80442000 & 0.00283000 \\ \mathrm{C} & 3.03319600 & 2.62695600 & -1.22147800 \\ \mathrm{C} & 1.78909900 & 1.99550600 & -1.28515900 \\ \mathrm{O} & 3.63233900 & 2.46944600 & 2.39524600 \\ \mathrm{C} & -1.17745400 & -1.54406000 & 0.11466200 \\ \mathrm{C} & -1.78909300 & -1.99551400 & 1.28517000 \\ \mathrm{C} & -3.03319200 & -2.62695900 & 1.22149300 \\ \mathrm{C} & -3.67622900 & -2.80441500 & -0.00281200\end{array}$




$\begin{array}{lrrr}\mathrm{C} & -3.05621400 & -2.35471600 & -1.16529400 \\ \mathrm{C} & -1.80419900 & -1.73963900 & -1.11318000 \\ \mathrm{O} & -3.63235700 & -2.46942900 & -2.39522700 \\ \mathrm{O} & -3.68169600 & -3.08762700 & 2.32756900 \\ \mathrm{H} & 0.44451000 & -0.75371700 & 1.24646800 \\ \mathrm{H} & 0.88973500 & -1.58247800 & -1.67636300 \\ \mathrm{O} & 3.68170500 & 3.08762000 & -2.32755200 \\ \mathrm{H} & -0.88974400 & 1.58246500 & 1.67636600 \\ \mathrm{H} & -2.43390700 & 0.94668100 & -1.75817200 \\ \mathrm{H} & -2.96992000 & 2.32326800 & 2.33943800 \\ \mathrm{H} & 2.96990800 & -2.32328400 & -2.33944400 \\ \mathrm{H} & 2.43392100 & -0.94667100 & 1.75816100 \\ \mathrm{H} & 1.36801000 & 1.39656000 & 2.04253000 \\ \mathrm{H} & 4.64909900 & 3.28206900 & 0.02250900 \\ \mathrm{H} & 1.30857200 & 1.84347600 & -2.24655700 \\ \mathrm{H} & 4.50498900 & 2.86689800 & 2.30798100 \\ \mathrm{H} & -1.30855900 & -1.84349000 & 2.24656500 \\ \mathrm{H} & -4.64910600 & -3.28205900 & -0.02248700 \\ \mathrm{H} & -1.36802100 & -1.39655500 & -2.04251900 \\ \mathrm{H} & -4.50500900 & -2.86687500 & -2.30795800 \\ \mathrm{H} & -3.15123200 & -2.90727800 & 3.11027600 \\ \mathrm{H} & 3.15124900 & 2.90726500 & -3.11026100 \\ \mathrm{H} & -5.38289500 & 2.64733100 & 1.90514300 \\ \mathrm{H} & -4.82611500 & 1.27479100 & -2.17642300 \\ \mathrm{H} & 5.38288600 & -2.64734400 & -1.90516300 \\ \mathrm{H} & 4.82613100 & -1.27477900 & 2.17639800\end{array}$

resveratrol dimer (C3-C8')<smiles></smiles>

$\begin{array}{lrrc}\text { Enthalpy }= & -1531.477449 & \text { Free Energy }= \\ 01 & & & \\ \mathrm{H} & 3.51207300 & 0.62267400 & 1.74214200 \\ \mathrm{C} & 3.36634000 & -0.15119400 & 0.99417700 \\ \mathrm{C} & 2.11483700 & -0.52442400 & 0.67355100 \\ \mathrm{H} & 1.97704400 & -1.31639600 & -0.05701400 \\ \mathrm{C} & 4.60958200 & -0.69835700 & 0.44254400 \\ \mathrm{C} & 7.05543300 & -1.69289400 & -0.52247800 \\ \mathrm{C} & 4.63319400 & -1.59528900 & -0.63208200 \\ \mathrm{C} & 5.82439100 & -0.29702800 & 1.02408800 \\ \mathrm{C} & 7.03381400 & -0.79519300 & 0.54364900 \\ \mathrm{C} & 5.84879300 & -2.08508700 & -1.10333400 \\ \mathrm{H} & 3.72891500 & -1.91943200 & -1.12846000 \\ \mathrm{H} & 5.82117400 & 0.39993300 & 1.85677200 \\ \mathrm{H} & 8.00898900 & -2.06447300 & -0.88050700 \\ \mathrm{C} & 0.86966200 & 0.01876700 & 1.20924500\end{array}$




$\begin{array}{lrrr}\mathrm{C} & -1.57451600 & 1.12557900 & 2.35741100 \\ \mathrm{C} & 0.87025700 & 1.12517900 & 2.17133300 \\ \mathrm{C} & -0.32045400 & -0.48963900 & 0.81523000 \\ \mathrm{C} & -1.63561600 & 0.02026600 & 1.29765800 \\ \mathrm{C} & -0.25188000 & 1.63602600 & 2.71363100 \\ \mathrm{H} & 1.82417000 & 1.55327100 & 2.45688900 \\ \mathrm{H} & -0.34676100 & -1.30954100 & 0.10351300 \\ \mathrm{H} & -0.22648000 & 2.45309900 & 3.42496400 \\ \mathrm{O} & -2.60512000 & 1.56358500 & 2.84433600 \\ \mathrm{O} & 8.24075900 & -0.44158000 & 1.07460000 \\ \mathrm{H} & 8.10124000 & 0.17377200 & 1.80144900 \\ \mathrm{O} & 5.79922900 & -2.95490300 & -2.15380500 \\ \mathrm{H} & 6.69273000 & -3.22066600 & -2.39347400 \\ \mathrm{H} & -2.18179500 & -0.81070900 & 1.76161800 \\ \mathrm{C} & -2.57781500 & 0.53361900 & 0.12532500 \\ \mathrm{H} & -3.46483900 & 0.88782300 & 0.65261000 \\ \mathrm{C} & -2.96998100 & -0.58937000 & -0.78910100 \\ \mathrm{H} & -2.42448300 & -0.67550800 & -1.72369000 \\ \mathrm{C} & -1.96148500 & 1.72518400 & -0.59072000 \\ \mathrm{C} & -0.79444300 & 3.97444200 & -1.78597300 \\ \mathrm{C} & -0.94758500 & 1.56779000 & -1.53262700 \\ \mathrm{C} & -2.39384000 & 3.00890500 & -0.24327600 \\ \mathrm{C} & -1.80726600 & 4.12540900 & -0.83909700 \\ \mathrm{C} & -0.36976600 & 2.69253900 & -2.12584400 \\ \mathrm{H} & -0.56923100 & 0.59466400 & -1.81456500 \\ \mathrm{H} & -3.16402800 & 3.13528700 & 0.51019100 \\ \mathrm{H} & -0.35833500 & 4.85860400 & -2.23747900 \\ \mathrm{C} & -3.93643900 & -1.51047200 & -0.54280200 \\ \mathrm{C} & -5.93720500 & -3.54813100 & -0.08078700 \\ \mathrm{C} & -4.19576400 & -2.56543900 & -1.51701700 \\ \mathrm{C} & -4.74454100 & -1.51850100 & 0.67285700 \\ \mathrm{C} & -5.67348200 & -2.46783700 & 0.89223600 \\ \mathrm{C} & -5.12376800 & -3.51756200 & -1.31039900 \\ \mathrm{H} & -3.60551700 & -2.55989900 & -2.42871000 \\ \mathrm{H} & -4.58479900 & -0.74180200 & 1.41266500 \\ \mathrm{H} & -6.27682000 & -2.48302200 & 1.79259100 \\ \mathrm{H} & -5.31666900 & -4.30310700 & -2.03164600 \\ \mathrm{O} & -2.18228300 & 5.40143400 & -0.53579900 \\ \mathrm{H} & -2.87863100 & 5.37812300 & 0.12876000 \\ \mathrm{O} & 0.61910800 & 2.46723300 & -3.03859500 \\ \mathrm{H} & 0.94780500 & 3.30869700 & -3.37091100 \\ \mathrm{O} & -6.78150400 & -4.41140900 & 0.12400200\end{array}$

di-ortho-tert-butyl resveratrol radical

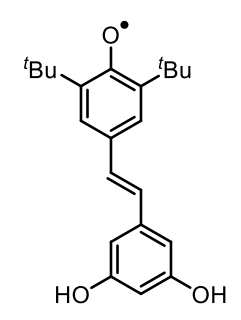

$\begin{array}{llll}\text { Enthalpy }=\quad-1080.122264 \quad \text { Free Energy }= & \begin{array}{l}-1080.204399 \\ \text { S65 }\end{array}\end{array}$ 


\begin{tabular}{|c|c|c|c|}
\hline & & & \\
\hline $\mathrm{C}$ & -2.23772700 & 1.28927800 & 0.00013900 \\
\hline $\mathrm{C}$ & -0.89426400 & 1.04300400 & 0.00004500 \\
\hline $\mathrm{C}$ & -0.35194100 & -0.27458700 & 0.00003200 \\
\hline $\mathrm{C}$ & -1.24973700 & -1.37741100 & 0.00012100 \\
\hline $\mathrm{C}$ & -2.61096500 & -1.23388100 & 0.00021800 \\
\hline $\mathrm{C}$ & -3.17194500 & 0.13655300 & 0.00022900 \\
\hline $\mathrm{O}$ & -4.40125400 & 0.31815400 & 0.00032000 \\
\hline $\mathrm{C}$ & 1.05580100 & -0.54141900 & -0.00006600 \\
\hline $\mathrm{C}$ & 2.06461300 & 0.37481400 & -0.00016000 \\
\hline $\mathrm{C}$ & 3.49288200 & 0.10002600 & -0.00025900 \\
\hline $\mathrm{C}$ & 4.37296900 & 1.19187000 & -0.00035900 \\
\hline $\mathrm{C}$ & 5.75274000 & 0.98612800 & -0.00045900 \\
\hline $\mathrm{C}$ & 6.27516200 & -0.30433000 & -0.00045900 \\
\hline $\mathrm{C}$ & 5.39934600 & -1.39568200 & -0.00035900 \\
\hline $\mathrm{C}$ & 4.02364900 & -1.20631900 & -0.00025900 \\
\hline $\mathrm{O}$ & 5.97620500 & -2.63202700 & -0.00036400 \\
\hline $\mathrm{O}$ & 6.54409600 & 2.09625300 & -0.00055400 \\
\hline $\mathrm{C}$ & -2.80721700 & 2.71410300 & 0.00015200 \\
\hline $\mathrm{C}$ & -1.69342100 & 3.77861200 & 0.00004400 \\
\hline $\mathrm{C}$ & -3.66900100 & 2.93431200 & 1.26779700 \\
\hline $\mathrm{C}$ & -3.66919400 & 2.93425800 & -1.26737100 \\
\hline $\mathrm{C}$ & -3.56340200 & -2.43605100 & 0.00031400 \\
\hline $\mathrm{C}$ & -2.80002900 & -3.77427100 & 0.00028500 \\
\hline $\mathrm{C}$ & -4.45276000 & -2.40040400 & -1.26696800 \\
\hline $\mathrm{C}$ & -4.45257300 & -2.40035100 & 1.26772500 \\
\hline $\mathrm{H}$ & -0.20287000 & 1.87268800 & -0.00002100 \\
\hline $\mathrm{H}$ & -0.80829800 & -2.36587900 & 0.00010900 \\
\hline $\mathrm{H}$ & 1.31869000 & -1.59564500 & -0.00006300 \\
\hline $\mathrm{H}$ & 1.80917300 & 1.42968200 & -0.00016500 \\
\hline $\mathrm{H}$ & 4.00049000 & 2.20853100 & -0.00036200 \\
\hline $\mathrm{H}$ & 7.34349900 & -0.48895900 & -0.00053500 \\
\hline $\mathrm{H}$ & 3.37001400 & -2.07084900 & -0.00018100 \\
\hline $\mathrm{H}$ & 5.28922100 & -3.30633400 & -0.00029000 \\
\hline $\mathrm{H}$ & 7.46993300 & 1.83260800 & -0.00061400 \\
\hline $\mathrm{H}$ & -1.05908500 & 3.70845700 & -0.88844000 \\
\hline $\mathrm{H}$ & -2.14791700 & 4.77230600 & 0.00005900 \\
\hline $\mathrm{H}$ & -1.05894800 & 3.70849400 & 0.88843300 \\
\hline $\mathrm{H}$ & -4.51571200 & 2.25176500 & 1.29098500 \\
\hline $\mathrm{H}$ & -4.04659500 & 3.96133800 & 1.28126000 \\
\hline $\mathrm{H}$ & -3.07010000 & 2.78312000 & 2.17091800 \\
\hline $\mathrm{H}$ & -4.04678800 & 3.96128400 & -1.28082200 \\
\hline $\mathrm{H}$ & -3.07042900 & 2.78302500 & -2.17057700 \\
\hline $\mathrm{H}$ & -4.51590900 & 2.25171200 & -1.29040100 \\
\hline $\mathrm{H}$ & -2.17173800 & -3.88775900 & 0.88854400 \\
\hline $\mathrm{H}$ & -3.51933800 & -4.59689900 & 0.00035600 \\
\hline $\mathrm{H}$ & -2.17187000 & -3.88779700 & -0.88806300 \\
\hline $\mathrm{H}$ & -5.06819200 & -1.50369300 & -1.28953200 \\
\hline $\mathrm{H}$ & -5.10882500 & -3.27619400 & -1.27996900 \\
\hline $\mathrm{H}$ & -3.83618700 & -2.42693900 & -2.17045100 \\
\hline $\mathrm{H}$ & -5.10863500 & -3.27614200 & 1.28086000 \\
\hline $\mathrm{H}$ & -3.83586700 & -2.42684700 & 2.17111800 \\
\hline $\mathrm{H}$ & -5.06800300 & -1.50364100 & 1.29034100 \\
\hline
\end{tabular}


simplified tert-butyl resveratrol dimer 5A (C8-C8', meso)

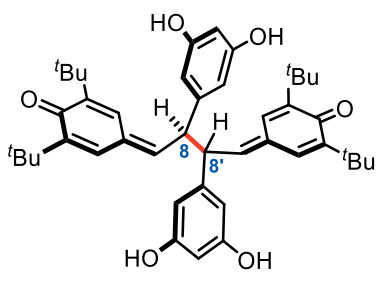

Enthalpy $=\quad-2160.281829 \quad$ Free Energy $=\quad-2160.416858$

01

$\begin{array}{llll}\text { C } & -2.70905000 & -0.71620600 & 0.84926300\end{array}$

$\begin{array}{llll}\mathrm{C} & -1.39572500 & -1.05406500 & 0.77481800\end{array}$

$\begin{array}{llll}\text { C } & -0.21123500 & -0.14493200 & 0.76998500\end{array}$

$\begin{array}{llll}\text { C } & 0.21123600 & 0.14493400 & -0.76998800\end{array}$

$\begin{array}{lllll}\mathrm{C} & 1.39572800 & 1.05406200 & -0.77483300\end{array}$

$\begin{array}{llll}\mathrm{C} & 2.70905200 & 0.71619600 & -0.84927000\end{array}$

$\begin{array}{llll}\mathrm{H} & -0.47544100 & 0.82701300 & 1.18955100\end{array}$

$\begin{array}{llll}\text { C } & -3.18566200 & 0.62924000 & 1.09494900\end{array}$

$\begin{array}{llll}\text { C } & -4.49346100 & 0.96573800 & 1.04731600\end{array}$

$\begin{array}{llll}\text { C } & -5.49760400 & -0.08951800 & 0.70904700\end{array}$

$\begin{array}{llll}\text { C } & -5.01646400 & -1.47547200 & 0.42685900\end{array}$

$\begin{array}{llll}\text { C } & -3.69905800 & -1.73670000 & 0.56045000\end{array}$

$\begin{array}{llll}\mathrm{C} & 3.69906500 & 1.73669500 & -0.56048900\end{array}$

$\begin{array}{llll}\mathrm{C} & 5.01647100 & 1.47546700 & -0.42689900\end{array}$

$\begin{array}{llll}\mathrm{C} & 5.49760300 & 0.08949900 & -0.70903200\end{array}$

$\begin{array}{llll}\mathrm{C} & 4.49345700 & -0.96575800 & -1.04728700\end{array}$

$\begin{array}{llll}\text { C } & 3.18565900 & -0.62925600 & -1.09492600\end{array}$

$\begin{array}{lllll}\mathrm{O} & -6.69779500 & 0.17709600 & 0.66775100\end{array}$

$\begin{array}{llll}\mathrm{O} & 6.69779100 & -0.17712400 & -0.66770200\end{array}$

$\begin{array}{llll}\text { C } & 0.98372800 & -0.69426300 & 1.53160300\end{array}$

$\begin{array}{llll}\mathrm{C} & 1.36878000 & -2.02753200 & 1.41392000\end{array}$

$\begin{array}{llll}\mathrm{C} & 2.54445500 & -2.46909400 & 2.02270100\end{array}$

$\begin{array}{llll}\text { C } & 3.35658800 & -1.59136800 & 2.73247600\end{array}$

$\begin{array}{llll}\text { C } & 2.96844900 & -0.25680500 & 2.84578600\end{array}$

$\begin{array}{llll}\text { C } & 1.78110600 & 0.18813400 & 2.26556200\end{array}$

$\begin{array}{llll}\mathrm{O} & 2.86191600 & -3.78662600 & 1.84994700\end{array}$

$\begin{array}{lllll}\mathrm{C} & & -0.98372900 & 0.69427500 & -1.53159700\end{array}$

$\begin{array}{llll}\text { C } & -1.78110300 & -0.18810800 & -2.26557700\end{array}$

$\begin{array}{lllll}\mathrm{C} & -2.96844800 & 0.25684000 & -2.84578900\end{array}$

$\begin{array}{lllll}\text { C } & -3.35659200 & 1.59139800 & -2.73244700\end{array}$

$\begin{array}{llll}\text { C } & -2.54446400 & 2.46911100 & -2.02265100\end{array}$

$\begin{array}{lllll}\mathrm{C} & -1.36878800 & 2.02753900 & -1.41387900\end{array}$

$\begin{array}{llll}\mathrm{O} & -2.86193100 & 3.78663700 & -1.84986800\end{array}$

O $\quad-3.80719700 \quad-0.57063500 \quad-3.53435000$

$\mathrm{H} \quad 0.47543300 \quad-0.82701200 \quad-1.18955700$

$\mathrm{H} \quad \begin{array}{llll}\mathrm{H} & 1.17828500 & 2.09925500 & -0.57581200\end{array}$

$\begin{array}{llll}\mathrm{O} & 3.80720200 & 0.57068200 & 3.53432800\end{array}$

$\begin{array}{lllll}\text { C } & 4.98883300 & -2.39110500 & -1.33363200\end{array}$

C $\quad 5.90751400 \quad-2.37772500-2.57906400$

$\begin{array}{llll}\text { C } & 3.82081600 & -3.35660400 & -1.61307300\end{array}$ 


\begin{tabular}{|c|c|c|c|}
\hline $\mathrm{C}$ & 5.76885300 & -2.94183400 & -0.11488900 \\
\hline $\mathrm{C}$ & 6.02960400 & 2.53441400 & 0.02793800 \\
\hline $\mathrm{C}$ & 6.70753900 & 2.05465100 & 1.33540100 \\
\hline $\mathrm{C}$ & 7.09433000 & 2.77142900 & -1.06869700 \\
\hline $\mathrm{C}$ & 5.34751100 & 3.88367900 & 0.32199800 \\
\hline $\mathrm{C}$ & -6.02959000 & -2.53440800 & -0.02802300 \\
\hline $\mathrm{C}$ & -5.34748500 & -3.88365100 & -0.32215300 \\
\hline $\mathrm{C}$ & -7.09430800 & -2.77148900 & 1.06860400 \\
\hline $\mathrm{C}$ & -6.70753500 & -2.05459000 & -1.33546100 \\
\hline $\mathrm{C}$ & -4.98884300 & 2.39107800 & 1.33368700 \\
\hline $\mathrm{C}$ & -5.76888100 & 2.94181700 & 0.11495900 \\
\hline $\mathrm{C}$ & -3.82082900 & 3.35658200 & 1.61312400 \\
\hline $\mathrm{C}$ & -5.90750900 & 2.37767600 & 2.57912900 \\
\hline $\mathrm{H}$ & -1.17827900 & -2.09925200 & 0.57576900 \\
\hline $\mathrm{H}$ & -2.44581600 & 1.38215100 & 1.31914800 \\
\hline $\mathrm{H}$ & -3.31462200 & -2.73415100 & 0.38658200 \\
\hline $\mathrm{H}$ & 3.31463400 & 2.73415300 & -0.38664900 \\
\hline $\mathrm{H}$ & 2.44581100 & -1.38217100 & -1.31910600 \\
\hline $\mathrm{H}$ & 0.79672000 & -2.74795000 & 0.84532200 \\
\hline $\mathrm{H}$ & 4.28671500 & -1.91175200 & 3.18680000 \\
\hline $\mathrm{H}$ & 1.50174700 & 1.23357600 & 2.34765400 \\
\hline $\mathrm{H}$ & 3.70841600 & -3.97695700 & 2.26736500 \\
\hline $\mathrm{H}$ & -1.50173900 & -1.23354700 & -2.34769300 \\
\hline $\mathrm{H}$ & -4.28672000 & 1.91178900 & -3.18676600 \\
\hline $\mathrm{H}$ & -0.79673200 & 2.74794500 & -0.84526200 \\
\hline $\mathrm{H}$ & -3.70844400 & 3.97696800 & -2.26726000 \\
\hline $\mathrm{H}$ & -3.47233400 & -1.47239900 & -3.49822200 \\
\hline $\mathrm{H}$ & 3.47235000 & 1.47244800 & 3.49816500 \\
\hline $\mathrm{H}$ & 6.78309000 & -1.75222700 & -2.41674100 \\
\hline $\mathrm{H}$ & 6.24277600 & -3.39567300 & -2.80069900 \\
\hline $\mathrm{H}$ & 5.36459900 & -2.00354900 & -3.45213000 \\
\hline $\mathrm{H}$ & 4.22285800 & -4.35053300 & -1.82549400 \\
\hline $\mathrm{H}$ & 3.15562200 & -3.45074800 & -0.75137500 \\
\hline $\mathrm{H}$ & 3.23636500 & -3.04933500 & -2.48537600 \\
\hline $\mathrm{H}$ & 5.13149800 & -2.93450000 & 0.77172800 \\
\hline $\mathrm{H}$ & 6.65776000 & -2.34992100 & 0.08885700 \\
\hline $\mathrm{H}$ & 6.07016100 & -3.97566600 & -0.30956300 \\
\hline $\mathrm{H}$ & 5.95677900 & 1.82823000 & 2.09881400 \\
\hline $\mathrm{H}$ & 7.36515900 & 2.83963100 & 1.72097900 \\
\hline $\mathrm{H}$ & 7.30101000 & 1.15894300 & 1.16409200 \\
\hline $\mathrm{H}$ & 7.64649900 & 1.85924500 & -1.28348900 \\
\hline $\mathrm{H}$ & 7.80135400 & 3.53836700 & -0.73740500 \\
\hline $\mathrm{H}$ & 6.62306200 & 3.12259000 & -1.99162500 \\
\hline $\mathrm{H}$ & 4.60349400 & 3.79965900 & 1.12042300 \\
\hline $\mathrm{H}$ & 6.10095700 & 4.60383300 & 0.64999500 \\
\hline $\mathrm{H}$ & 4.85953600 & 4.30005500 & -0.56399100 \\
\hline $\mathrm{H}$ & -4.85950300 & -4.30006800 & 0.56381400 \\
\hline $\mathrm{H}$ & -6.10092400 & -4.60379700 & -0.65018400 \\
\hline $\mathrm{H}$ & -4.60347100 & -3.79958300 & -1.12057600 \\
\hline $\mathrm{H}$ & -7.64648700 & -1.85932200 & 1.28344200 \\
\hline $\mathrm{H}$ & -7.80132400 & -3.53842000 & 0.73727800 \\
\hline $\mathrm{H}$ & -6.62303200 & -3.12268800 & 1.99151300 \\
\hline $\mathrm{H}$ & -5.95678000 & -1.82812800 & -2.09886700 \\
\hline $\mathrm{H}$ & -7.30101500 & -1.15889600 & -1.16410800 \\
\hline
\end{tabular}




$\begin{array}{lrrr}\mathrm{H} & -7.36514900 & -2.83955800 & -1.72107200 \\ \mathrm{H} & -6.07018900 & 3.97564600 & 0.30964800 \\ \mathrm{H} & -5.13153900 & 2.93449300 & -0.77166700 \\ \mathrm{H} & -6.65779000 & 2.34990400 & -0.08878000 \\ \mathrm{H} & -3.15564800 & 3.45074100 & 0.75141700 \\ \mathrm{H} & -4.22287500 & 4.35050500 & 1.82556400 \\ \mathrm{H} & -3.23636300 & 3.04930700 & 2.48541500 \\ \mathrm{H} & -6.24277800 & 3.39561800 & 2.80077900 \\ \mathrm{H} & -6.78308000 & 1.75217100 & 2.41680900 \\ \mathrm{H} & -5.36458000 & 2.00349500 & 3.45218400\end{array}$

simplified tert-butyl resveratrol dimer 5B (C3-C8')

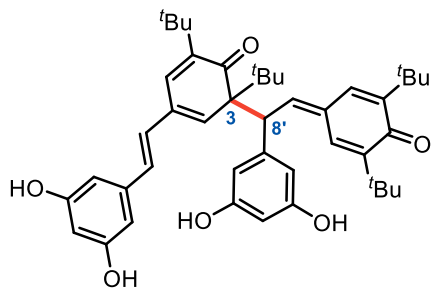

\begin{tabular}{|c|c|c|c|}
\hline Enthalpy= & \multicolumn{2}{|c|}{-2160.259756} & Free Energy= \\
\hline 01 & & & \\
\hline $\mathrm{C}$ & 2.71055100 & -2.41548300 & -1.79167000 \\
\hline $\mathrm{C}$ & 1.76856500 & -1.45514700 & -1.68007200 \\
\hline $\mathrm{C}$ & 1.97730000 & -0.21205700 & -0.95988400 \\
\hline $\mathrm{C}$ & 3.28257000 & 0.01315700 & -0.37539200 \\
\hline $\mathrm{C}$ & 4.28204300 & -0.89250000 & -0.43818000 \\
\hline $\mathrm{C}$ & 4.05046800 & -2.17379500 & -1.17243600 \\
\hline $\mathrm{O}$ & 4.94263700 & -3.01614100 & -1.26180800 \\
\hline $\mathrm{C}$ & 0.92913600 & 0.63687700 & -0.80745800 \\
\hline $\mathrm{C}$ & 0.88688400 & 1.92226400 & -0.02298600 \\
\hline $\mathrm{C}$ & 0.49764100 & 3.07188000 & -0.94513600 \\
\hline $\mathrm{C}$ & 1.34323000 & 4.17472800 & -1.03188300 \\
\hline $\mathrm{C}$ & 1.02761200 & 5.24121600 & -1.87864400 \\
\hline $\mathrm{C}$ & -0.12737700 & 5.21417500 & -2.65483400 \\
\hline $\mathrm{C}$ & -0.95631200 & 4.09404700 & -2.59162800 \\
\hline $\mathrm{C}$ & -0.65026500 & 3.02650800 & -1.74915400 \\
\hline $\mathrm{O}$ & -2.06340100 & 4.10427000 & -3.39007400 \\
\hline $\mathrm{O}$ & 1.90005000 & 6.28812600 & -1.90051700 \\
\hline $\mathrm{C}$ & -0.00690300 & 1.68687200 & 1.29004000 \\
\hline $\mathrm{C}$ & -1.41546700 & 1.38805500 & 0.89134900 \\
\hline $\mathrm{C}$ & -2.03165000 & 0.20451800 & 1.09397300 \\
\hline $\mathrm{C}$ & -1.31049400 & -0.86712500 & 1.77298500 \\
\hline $\mathrm{C}$ & -0.03473600 & -0.78187200 & 2.21197700 \\
\hline $\mathrm{C}$ & 0.70547000 & 0.48322900 & 1.97241700 \\
\hline $\mathrm{O}$ & 1.88390400 & 0.60158000 & 2.27540900 \\
\hline $\mathrm{C}$ & -3.40773400 & 0.01532700 & 0.63483600 \\
\hline $\mathrm{C}$ & -4.11451700 & -1.12872000 & 0.63556500 \\
\hline $\mathrm{C}$ & -5.49056900 & -1.30209100 & 0.15986100 \\
\hline $\mathrm{C}$ & -5.95747800 & -2.60227800 & -0.05713000 \\
\hline $\mathrm{C}$ & -7.25459300 & -2.81543600 & -0.52815100 \\
\hline $\mathrm{C}$ & -8.10310800 & -1.74117700 & -0.77894700 \\
\hline
\end{tabular}




\begin{tabular}{|c|c|c|c|}
\hline $\mathrm{C}$ & -7.64131200 & -0.44240400 & -0.54794600 \\
\hline $\mathrm{C}$ & -6.35044300 & -0.21566000 & -0.08331000 \\
\hline $\mathrm{O}$ & -8.52461400 & 0.56894800 & -0.79580900 \\
\hline $\mathrm{O}$ & -7.63599800 & -4.11028300 & -0.72378600 \\
\hline $\mathrm{H}$ & 0.78473000 & -1.59389400 & -2.11089400 \\
\hline $\mathrm{H}$ & 3.42455200 & 0.93178400 & 0.17021200 \\
\hline $\mathrm{H}$ & -0.01187300 & 0.34266700 & -1.26295000 \\
\hline $\mathrm{H}$ & 1.87793600 & 2.13762300 & 0.36568300 \\
\hline $\mathrm{H}$ & 2.25746400 & 4.23158000 & -0.45659200 \\
\hline $\mathrm{H}$ & -0.38978000 & 6.02891500 & -3.32026500 \\
\hline $\mathrm{H}$ & -1.30296600 & 2.16200700 & -1.73719100 \\
\hline $\mathrm{H}$ & -2.53367000 & 3.26989400 & -3.29361100 \\
\hline $\mathrm{H}$ & 1.59346200 & 6.95059800 & -2.52817000 \\
\hline $\mathrm{H}$ & -1.95908700 & 2.18319000 & 0.39966500 \\
\hline $\mathrm{H}$ & -1.85434900 & -1.78661800 & 1.93521700 \\
\hline $\mathrm{H}$ & -3.64537400 & -2.04144200 & 0.99043700 \\
\hline $\mathrm{H}$ & -5.32458600 & -3.46191100 & 0.12482800 \\
\hline $\mathrm{H}$ & -9.11651700 & -1.88351600 & -1.13698600 \\
\hline $\mathrm{H}$ & -6.02771700 & 0.80001700 & 0.11732300 \\
\hline $\mathrm{H}$ & -8.10966700 & 1.41238700 & -0.58879400 \\
\hline $\mathrm{H}$ & -8.54362000 & -4.13312600 & -1.04368900 \\
\hline $\mathrm{C}$ & 2.45378300 & -3.74761100 & -2.51033500 \\
\hline $\mathrm{C}$ & 2.63478000 & -4.91875800 & -1.51433000 \\
\hline $\mathrm{C}$ & 1.02060900 & -3.82582200 & -3.06934800 \\
\hline $\mathrm{C}$ & 3.43331300 & -3.90746600 & -3.69750600 \\
\hline $\mathrm{H}$ & 3.65221700 & -4.95671600 & -1.13102200 \\
\hline $\mathrm{H}$ & 1.94626200 & -4.81494700 & -0.67004600 \\
\hline $\mathrm{H}$ & 2.41486200 & -5.86663800 & -2.01515200 \\
\hline $\mathrm{H}$ & 0.82516400 & -3.04129200 & -3.80623700 \\
\hline $\mathrm{H}$ & 0.88179400 & -4.78772600 & -3.56911000 \\
\hline $\mathrm{H}$ & 0.26809400 & -3.75483900 & -2.27830400 \\
\hline $\mathrm{H}$ & 3.22618500 & -4.84481400 & -4.22321500 \\
\hline $\mathrm{H}$ & 3.30959800 & -3.08625500 & -4.40992900 \\
\hline $\mathrm{H}$ & 4.46615700 & -3.92132800 & -3.35634300 \\
\hline $\mathrm{C}$ & 5.63713600 & -0.65950900 & 0.24489100 \\
\hline $\mathrm{C}$ & 5.67584400 & 0.69667000 & 0.97544900 \\
\hline $\mathrm{C}$ & 5.88704100 & -1.76575200 & 1.29899700 \\
\hline $\mathrm{C}$ & 6.76987000 & -0.66831700 & -0.80863900 \\
\hline $\mathrm{H}$ & 5.54901500 & 1.53583500 & 0.28480100 \\
\hline $\mathrm{H}$ & 4.90720000 & 0.76429500 & 1.75031700 \\
\hline $\mathrm{H}$ & 6.64870100 & 0.81524700 & 1.45913300 \\
\hline $\mathrm{H}$ & 5.92628100 & -2.75034700 & 0.83779300 \\
\hline $\mathrm{H}$ & 6.83895100 & -1.58042500 & 1.80603800 \\
\hline $\mathrm{H}$ & 5.09633600 & -1.76177900 & 2.05451400 \\
\hline $\mathrm{H}$ & 7.72897600 & -0.46958200 & -0.32027700 \\
\hline $\mathrm{H}$ & 6.83030800 & -1.62971500 & -1.31459200 \\
\hline $\mathrm{H}$ & 6.60403000 & 0.11289700 & -1.55668500 \\
\hline $\mathrm{C}$ & 0.68308100 & -1.94509700 & 2.91155900 \\
\hline $\mathrm{C}$ & 1.16889900 & -1.49713800 & 4.31076000 \\
\hline $\mathrm{H}$ & 1.65011700 & -2.33786400 & 4.81894100 \\
\hline $\mathrm{H}$ & 1.88782400 & -0.68342300 & 4.23882600 \\
\hline $\mathrm{H}$ & 0.32575600 & -1.16893100 & 4.92624000 \\
\hline $\mathrm{C}$ & 1.88810900 & -2.40496400 & 2.05616000 \\
\hline $\mathrm{H}$ & 1.56279900 & -2.71752600 & 1.06050400 \\
\hline
\end{tabular}




$\begin{array}{lrrr}\mathrm{H} & 2.62057100 & -1.61097600 & 1.93905000 \\ \mathrm{H} & 2.37626400 & -3.25842500 & 2.53583400 \\ \mathrm{C} & -0.24833500 & -3.15668900 & 3.10123800 \\ \mathrm{H} & -1.12201100 & -2.91240400 & 3.71294100 \\ \mathrm{H} & -0.59299900 & -3.56115200 & 2.14494300 \\ \mathrm{H} & 0.29910100 & -3.95223600 & 3.61204600 \\ \mathrm{C} & -0.01164900 & 2.92020700 & 2.32859900 \\ \mathrm{C} & 1.38560200 & 3.53194400 & 2.54059900 \\ \mathrm{H} & 1.33050000 & 4.25148700 & 3.36298800 \\ \mathrm{H} & 2.12725000 & 2.77796400 & 2.79489100 \\ \mathrm{H} & 1.72188400 & 4.07834700 & 1.65950900 \\ \mathrm{C} & -0.95956300 & 4.05069800 & 1.87065900 \\ \mathrm{H} & -0.83300200 & 4.90496900 & 2.54119400 \\ \mathrm{H} & -0.74286800 & 4.39050800 & 0.85811500 \\ \mathrm{H} & -2.00911100 & 3.75319500 & 1.92228500 \\ \mathrm{C} & -0.52875800 & 2.39084800 & 3.68499400 \\ \mathrm{H} & -0.69514400 & 3.22913700 & 4.36707600 \\ \mathrm{H} & -1.47705000 & 1.85887100 & 3.57012800 \\ \mathrm{H} & 0.19014500 & 1.71880000 & 4.15778500 \\ \mathrm{H} & -3.86935000 & 0.91353400 & 0.23204000\end{array}$

di-ortho-TMS resveratrol radical<smiles>CSc1cc(/C=C/c2cc(O)cc(O)c2)cc([As](C)(=O)=O)c1[O]</smiles>

$\begin{array}{lrrr}\text { Enthalpy }= & -1583.020820 & \text { Free Energy= } \\ 02 & & & \\ \mathrm{C} & -1.87055300 & 1.26821400 & 0.00011300 \\ \mathrm{C} & -0.52478200 & 1.02250300 & 0.00001800 \\ \mathrm{C} & 0.01628000 & -0.30281100 & 0.00000600 \\ \mathrm{C} & -0.89891700 & -1.39955700 & 0.00009600 \\ \mathrm{C} & -2.25979700 & -1.23638700 & 0.00019300 \\ \mathrm{C} & -2.79969300 & 0.12861300 & 0.00020500 \\ \mathrm{O} & -4.03317300 & 0.32098200 & 0.00029800 \\ \mathrm{C} & 1.41992700 & -0.58105200 & -0.00009200 \\ \mathrm{C} & 2.43579500 & 0.32885200 & -0.00018400 \\ \mathrm{C} & 3.86135300 & 0.04407100 & -0.00028400 \\ \mathrm{C} & 4.74878400 & 1.13015100 & -0.00036500 \\ \mathrm{C} & 6.12709500 & 0.91481500 & -0.00046300 \\ \mathrm{C} & 6.63999400 & -0.37951600 & -0.00048100 \\ \mathrm{C} & 5.75685300 & -1.46518300 & -0.00040000 \\ \mathrm{C} & 4.38259100 & -1.26628800 & -0.00030300 \\ \mathrm{O} & 6.32568100 & -2.70483600 & -0.00042400 \\ \mathrm{O} & 6.92597000 & 2.01907600 & -0.00053700 \\ \mathrm{C} & -1.20529600 & 4.25855800 & -0.00001600 \\ \mathrm{C} & -3.67161100 & 3.22919800 & 1.54956600 \\ \mathrm{C} & -3.67186300 & 3.22912100 & -1.54914500\end{array}$




$\begin{array}{lrrr}\mathrm{C} & -2.52494000 & -4.28818300 & 0.00026900 \\ \mathrm{C} & -4.56724000 & -2.56407700 & -1.54875100 \\ \mathrm{C} & -4.56701400 & -2.56402200 & 1.54952500 \\ \mathrm{H} & 0.16579200 & 1.85887700 & -0.00005000 \\ \mathrm{H} & -0.47394400 & -2.40027600 & 0.00008500 \\ \mathrm{H} & 1.67512400 & -1.63730800 & -0.00008900 \\ \mathrm{H} & 2.18693900 & 1.38523700 & -0.00018500 \\ \mathrm{H} & 4.38318800 & 2.14928600 & -0.00035300 \\ \mathrm{H} & 7.70699600 & -0.57172000 & -0.00055600 \\ \mathrm{H} & 3.72283100 & -2.12608500 & -0.00024300 \\ \mathrm{H} & 5.63490400 & -3.37529900 & -0.00036300 \\ \mathrm{H} & 7.85015100 & 1.74959100 & -0.00060100 \\ \mathrm{H} & -0.57174800 & 4.15362400 & -0.88613100 \\ \mathrm{H} & -1.60226000 & 5.27827600 & -0.00000900 \\ \mathrm{H} & -0.57160200 & 4.15366700 & 0.88600000 \\ \mathrm{H} & -4.46479200 & 2.47889500 & 1.56059200 \\ \mathrm{H} & -4.12921100 & 4.22296500 & 1.57841400 \\ \mathrm{H} & -3.07072300 & 3.10747900 & 2.45551600 \\ \mathrm{H} & -4.12946700 & 4.22288700 & -1.57797000 \\ \mathrm{H} & -3.07112300 & 3.10735500 & -2.45518700 \\ \mathrm{H} & -4.46504600 & 2.47881700 & -1.56000300 \\ \mathrm{H} & -1.88878500 & -4.37799300 & 0.88615100 \\ \mathrm{H} & -3.21012300 & -5.14139800 & 0.00033500 \\ \mathrm{H} & -1.88891300 & -4.37802500 & -0.88570100 \\ \mathrm{H} & -5.09679400 & -1.60926200 & -1.56011700 \\ \mathrm{H} & -5.30359700 & -3.37328800 & -1.57652300 \\ \mathrm{H} & -3.95818200 & -2.62998700 & -2.45501500 \\ \mathrm{H} & -5.30336500 & -3.37323400 & 1.57743400 \\ \mathrm{H} & -3.95782400 & -2.62989700 & 2.45570300 \\ \mathrm{H} & -5.09656900 & -1.60920800 & 1.56093400 \\ \mathrm{Si} & -2.62478700 & 3.01037800 & 0.00013100 \\ \mathrm{Si} & -3.50246100 & -2.67022600 & 0.00031100\end{array}$

simplified TMS resveratrol dimer 5A (C8-C8', meso)

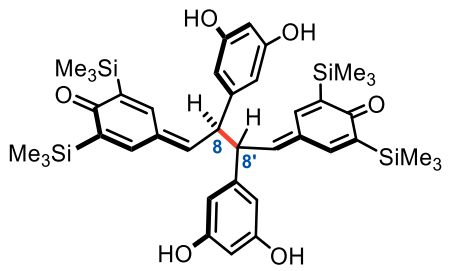

$\begin{array}{lrrr}\text { Enthalpy }= & -3166.081932 & \text { Free Energy }= \\ 01 & & & \\ \text { C } & -2.68040100 & 0.60606900 & -0.97111900 \\ \text { C } & -1.36862800 & 0.95349500 & -0.93370300 \\ \text { C } & -0.18821600 & 0.05122100 & -0.79018100 \\ \text { C } & 0.18821800 & -0.05121200 & 0.79017500 \\ \text { C } & 1.36862600 & -0.95349100 & 0.93369900 \\ \text { C } & 2.68040100 & -0.60607000 & 0.97111000 \\ \text { H } & -0.44628100 & -0.96428700 & -1.09427300 \\ \text { C } & -3.15960700 & -0.76639100 & -1.03673400\end{array}$




\begin{tabular}{|c|c|c|c|}
\hline $\mathrm{C}$ & -4.46709300 & -1.09050500 & -0.90738700 \\
\hline $\mathrm{C}$ & -5.45842700 & -0.01305100 & -0.68256700 \\
\hline $\mathrm{C}$ & -4.99372200 & 1.39082600 & -0.60527800 \\
\hline $\mathrm{C}$ & -3.68122500 & 1.65132800 & -0.79909200 \\
\hline $\mathrm{C}$ & 3.68122100 & -1.65133400 & 0.79908700 \\
\hline $\mathrm{C}$ & 4.99371800 & -1.39083700 & 0.60527400 \\
\hline $\mathrm{C}$ & 5.45843000 & 0.01303800 & 0.68256200 \\
\hline $\mathrm{C}$ & 4.46709900 & 1.09049800 & 0.90737300 \\
\hline $\mathrm{C}$ & 3.15961200 & 0.76638900 & 1.03671800 \\
\hline $\mathrm{O}$ & -6.65823500 & -0.28197600 & -0.56265000 \\
\hline $\mathrm{O}$ & 6.65824000 & 0.28195500 & 0.56265300 \\
\hline $\mathrm{C}$ & 1.02787800 & 0.50318200 & -1.58134600 \\
\hline $\mathrm{C}$ & 1.41078000 & 1.84157700 & -1.62609200 \\
\hline $\mathrm{C}$ & 2.60159700 & 2.20469200 & -2.25769900 \\
\hline $\mathrm{C}$ & 3.43266000 & 1.24494700 & -2.82542100 \\
\hline $\mathrm{C}$ & 3.04738700 & -0.09438200 & -2.77401900 \\
\hline $\mathrm{C}$ & 1.84389900 & -0.46402200 & -2.17471000 \\
\hline $\mathrm{O}$ & 2.90927800 & 3.53547300 & -2.25480600 \\
\hline $\mathrm{C}$ & -1.02787600 & -0.50316600 & 1.58134100 \\
\hline $\mathrm{C}$ & -1.84388600 & 0.46404100 & 2.17471500 \\
\hline $\mathrm{C}$ & -3.04737600 & 0.09440800 & 2.77402500 \\
\hline $\mathrm{C}$ & -3.43266200 & -1.24491700 & 2.82541600 \\
\hline $\mathrm{C}$ & -2.60161300 & -2.20466700 & 2.25768000 \\
\hline $\mathrm{C}$ & -1.41079200 & -1.84155700 & 1.62607600 \\
\hline $\mathrm{O}$ & -2.90930900 & -3.53544300 & 2.25477300 \\
\hline $\mathrm{O}$ & -3.90430900 & 1.00305300 & 3.32375400 \\
\hline $\mathrm{H}$ & 0.44628800 & 0.96429600 & 1.09426600 \\
\hline $\mathrm{H}$ & 1.15250200 & -2.01613500 & 0.87157000 \\
\hline $\mathrm{O}$ & 3.90433100 & -1.00302300 & -3.32373700 \\
\hline $\mathrm{C}$ & 6.26947600 & 3.03403000 & 2.47260400 \\
\hline $\mathrm{C}$ & 3.65829100 & 4.03816800 & 1.14388400 \\
\hline $\mathrm{C}$ & 6.07691500 & 3.23843600 & -0.60362500 \\
\hline $\mathrm{C}$ & 7.00040300 & -2.20207300 & -1.54200600 \\
\hline $\mathrm{C}$ & 7.62002600 & -2.81692900 & 1.43809200 \\
\hline $\mathrm{C}$ & 5.37938500 & -4.35544700 & -0.03187600 \\
\hline $\mathrm{C}$ & -5.37939900 & 4.35543600 & 0.03187200 \\
\hline C & -7.62004300 & 2.81690100 & -1.43807600 \\
\hline $\mathrm{C}$ & -7.00039200 & 2.20205300 & 1.54201800 \\
\hline $\mathrm{C}$ & -6.07689900 & -3.23842000 & 0.60364200 \\
\hline $\mathrm{C}$ & -3.65828200 & -4.03817700 & -1.14387800 \\
\hline $\mathrm{C}$ & -6.26947700 & -3.03406400 & -2.47258800 \\
\hline $\mathrm{H}$ & -1.15251000 & 2.01614000 & -0.87156600 \\
\hline $\mathrm{H}$ & -2.42484500 & -1.54875700 & -1.18556500 \\
\hline $\mathrm{H}$ & -3.31017700 & 2.67297200 & -0.75783800 \\
\hline $\mathrm{H}$ & 3.31016900 & -2.67297700 & 0.75783700 \\
\hline $\mathrm{H}$ & 2.42485200 & 1.54875700 & 1.18554400 \\
\hline $\mathrm{H}$ & 0.82487800 & 2.62805400 & -1.17030900 \\
\hline $\mathrm{H}$ & 4.37440200 & 1.50637200 & -3.29305600 \\
\hline $\mathrm{H}$ & 1.56616700 & -1.51211900 & -2.12939000 \\
\hline $\mathrm{H}$ & 3.76699800 & 3.67717000 & -2.66882600 \\
\hline $\mathrm{H}$ & -1.56614400 & 1.51213600 & 2.12940200 \\
\hline $\mathrm{H}$ & -4.37440300 & -1.50633600 & 3.29305800 \\
\hline $\mathrm{H}$ & -0.82490100 & -2.62803500 & 1.17028100 \\
\hline $\mathrm{H}$ & -3.76706000 & -3.67712900 & 2.66873200 \\
\hline
\end{tabular}




$\begin{array}{lrrr}\mathrm{H} & -3.56626400 & 1.89325800 & 3.18248200 \\ \mathrm{H} & 3.56629900 & -1.89323100 & -3.18245000 \\ \mathrm{H} & 7.08790700 & 2.31571500 & 2.38850200 \\ \mathrm{H} & 6.69518600 & 4.04054700 & 2.53193700 \\ \mathrm{H} & 5.73453600 & 2.83797600 & 3.40639000 \\ \mathrm{H} & 4.01220700 & 5.06758400 & 1.25774700 \\ \mathrm{H} & 3.02797000 & 4.00283900 & 0.25159400 \\ \mathrm{H} & 3.04505300 & 3.80616000 & 2.02014100 \\ \mathrm{H} & 5.46049600 & 3.01625800 & -1.47755600 \\ \mathrm{H} & 6.96664400 & 2.60916700 & -0.65576300 \\ \mathrm{H} & 6.37778100 & 4.28937100 & -0.65113000 \\ \mathrm{H} & 6.20914700 & -1.98135100 & -2.26470700 \\ \mathrm{H} & 7.63724000 & -2.99083000 & -1.95408200 \\ \mathrm{H} & 7.59839000 & -1.29640000 & -1.42122800 \\ \mathrm{H} & 8.09383200 & -1.84063600 & 1.55987100 \\ \mathrm{H} & 8.38699200 & -3.54419700 & 1.15418100 \\ \mathrm{H} & 7.20600300 & -3.12118200 & 2.40377900 \\ \mathrm{H} & 4.59492600 & -4.32282500 & -0.79465700 \\ \mathrm{H} & 6.08446000 & -5.14062400 & -0.32106900 \\ \mathrm{H} & 4.91876900 & -4.66069000 & 0.91267200 \\ \mathrm{H} & -4.91879400 & 4.66068000 & -0.91268100 \\ \mathrm{H} & -6.08447700 & 5.14060800 & 0.32107000 \\ \mathrm{H} & -4.59493300 & 4.32281900 & 0.79464600 \\ \mathrm{H} & -8.09385000 & 1.84060700 & -1.55984400 \\ \mathrm{H} & -8.38700800 & 3.54417200 & -1.15416500 \\ \mathrm{H} & -7.20602800 & 3.12114800 & -2.40376800 \\ \mathrm{H} & -6.20912900 & 1.98134300 & 2.26471500 \\ \mathrm{H} & -7.59837000 & 1.29637200 & 1.42124600 \\ \mathrm{H} & -7.63723700 & 2.99080400 & 1.95409600 \\ \mathrm{H} & -6.37759300 & -4.28939900 & 0.65126300 \\ \mathrm{H} & -5.46055200 & -3.01602700 & 1.47757000 \\ \mathrm{H} & -6.96673100 & -2.60928900 & 0.65567800 \\ \mathrm{H} & -3.02795700 & -4.00284600 & -0.25159000 \\ \mathrm{H} & -4.01219800 & -5.06759300 & -1.25773800 \\ \mathrm{H} & -3.04504600 & -3.80617100 & -2.02013700 \\ \mathrm{H} & -6.69520000 & -4.04057700 & -2.53189100 \\ \mathrm{H} & -7.08790000 & -2.31573800 & -2.38849700 \\ \mathrm{H} & -5.73454200 & -2.83804000 & -3.40638200 \\ \mathrm{Si} & 6.27827400 & -2.69849200 & 0.12482500 \\ \mathrm{Si} & 5.12929100 & 2.86614000 & 0.98387200 \\ \mathrm{Si} & -5.12928200 & -2.86614900 & -0.98386700 \\ \mathrm{Si} & -6.27828000 & 2.69847500 & -0.12481900\end{array}$

simplified TMS resveratrol dimer 5B (C3-C8')

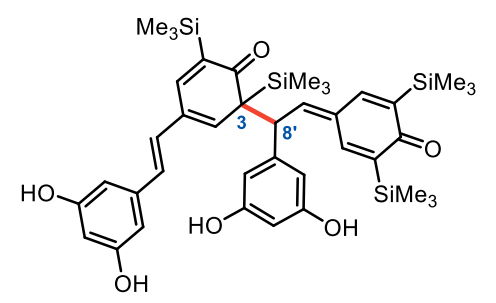

Enthalpy $=\quad-3166.070238 \quad$ Free Energy $=\quad-3166.234398$ 


\begin{tabular}{|c|c|c|c|}
\hline & & & \\
\hline $\mathrm{C}$ & 5.52692700 & 0.19612000 & -0.15477300 \\
\hline $\mathrm{C}$ & 4.33618100 & 0.78981400 & 0.09594200 \\
\hline $\mathrm{C}$ & 3.10027200 & 0.04625800 & 0.30293700 \\
\hline $\mathrm{C}$ & 3.19699800 & -1.40517300 & 0.29581400 \\
\hline $\mathrm{C}$ & 4.35441900 & -2.06358300 & 0.05236900 \\
\hline $\mathrm{C}$ & 5.58857200 & -1.28237200 & -0.20421800 \\
\hline $\mathrm{O}$ & 6.65167900 & -1.86182100 & -0.45134100 \\
\hline $\mathrm{C}$ & 1.93480800 & 0.73200100 & 0.43166700 \\
\hline $\mathrm{C}$ & 0.56370200 & 0.14478000 & 0.58232900 \\
\hline $\mathrm{C}$ & 0.00742200 & 0.42316600 & 1.98141700 \\
\hline $\mathrm{C}$ & -0.41208000 & -0.64676100 & 2.76586200 \\
\hline $\mathrm{C}$ & -0.98280700 & -0.40808000 & 4.01984600 \\
\hline $\mathrm{C}$ & -1.14264300 & 0.89154400 & 4.49246200 \\
\hline $\mathrm{C}$ & -0.71154200 & 1.95753800 & 3.70185300 \\
\hline $\mathrm{C}$ & -0.12309700 & 1.73201100 & 2.45958000 \\
\hline $\mathrm{O}$ & -0.90266100 & 3.21466100 & 4.19875900 \\
\hline $\mathrm{O}$ & -1.37885500 & -1.50083600 & 4.73537100 \\
\hline $\mathrm{C}$ & -0.48815100 & 0.59081500 & -0.51307300 \\
\hline $\mathrm{C}$ & -1.69154700 & -0.26366800 & -0.38391200 \\
\hline $\mathrm{C}$ & -2.96892400 & 0.18988200 & -0.46671400 \\
\hline $\mathrm{C}$ & -3.17015300 & 1.62646600 & -0.56100300 \\
\hline $\mathrm{C}$ & -2.16231100 & 2.53611900 & -0.59125300 \\
\hline $\mathrm{C}$ & -0.76643000 & 2.07918100 & -0.53837800 \\
\hline $\mathrm{O}$ & 0.15588000 & 2.90232500 & -0.52152000 \\
\hline $\mathrm{C}$ & -4.08807100 & -0.74672600 & -0.40590700 \\
\hline $\mathrm{C}$ & -5.38920000 & -0.46279000 & -0.59380000 \\
\hline $\mathrm{C}$ & -6.50760100 & -1.40792100 & -0.53052000 \\
\hline $\mathrm{C}$ & -7.75526600 & -0.98967300 & -1.00480800 \\
\hline $\mathrm{C}$ & -8.84702900 & -1.85946600 & -0.97833900 \\
\hline $\mathrm{C}$ & -8.71350500 & -3.14970300 & -0.47436400 \\
\hline $\mathrm{C}$ & -7.46962800 & -3.56371600 & 0.01064400 \\
\hline $\mathrm{C}$ & -6.37355900 & -2.70869500 & -0.01207500 \\
\hline $\mathrm{O}$ & -7.40354700 & -4.83459300 & 0.50563600 \\
\hline $\mathrm{O}$ & -10.02965100 & -1.38268700 & -1.46432200 \\
\hline $\mathrm{H}$ & 4.25553300 & 1.87353600 & 0.12786100 \\
\hline $\mathrm{H}$ & 2.29567000 & -1.97210400 & 0.50069000 \\
\hline $\mathrm{H}$ & 1.97219700 & 1.81397800 & 0.40335900 \\
\hline $\mathrm{H}$ & 0.62969900 & -0.93915100 & 0.48613200 \\
\hline $\mathrm{H}$ & -0.31823200 & -1.67065000 & 2.42587500 \\
\hline $\mathrm{H}$ & -1.59555000 & 1.09702800 & 5.45600500 \\
\hline $\mathrm{H}$ & 0.20912600 & 2.57261200 & 1.86661900 \\
\hline $\mathrm{H}$ & -0.59928500 & 3.86238500 & 3.55285700 \\
\hline $\mathrm{H}$ & -1.74964500 & -1.21511100 & 5.57657600 \\
\hline $\mathrm{H}$ & -1.51959200 & -1.32624800 & -0.23556500 \\
\hline $\mathrm{H}$ & -4.19441600 & 1.98441300 & -0.55721500 \\
\hline $\mathrm{H}$ & -5.67740000 & 0.55476700 & -0.84094800 \\
\hline $\mathrm{H}$ & -7.89605500 & 0.00607700 & -1.40647900 \\
\hline $\mathrm{H}$ & -9.54888300 & -3.83987700 & -0.43843000 \\
\hline $\mathrm{H}$ & -5.42750700 & -3.04671300 & 0.39590600 \\
\hline $\mathrm{H}$ & -6.50856900 & -5.01011400 & 0.81340000 \\
\hline $\mathrm{H}$ & -10.70393600 & -2.06596200 & -1.39329200 \\
\hline $\mathrm{C}$ & 7.76511800 & 0.74182300 & -2.19691000 \\
\hline
\end{tabular}




\begin{tabular}{|c|c|c|c|}
\hline $\mathrm{C}$ & 6.76084300 & 2.99272800 & -0.33538400 \\
\hline $\mathrm{C}$ & 8.41002900 & 0.65402300 & 0.83097200 \\
\hline $\mathrm{H}$ & 7.93965200 & -0.33292200 & -2.27955400 \\
\hline $\mathrm{H}$ & 7.03626600 & 1.03142200 & -2.95967000 \\
\hline $\mathrm{H}$ & 8.70247100 & 1.26529100 & -2.40947900 \\
\hline $\mathrm{H}$ & 6.38454100 & 3.25970000 & 0.65683800 \\
\hline $\mathrm{H}$ & 7.67000500 & 3.57613100 & -0.51019700 \\
\hline $\mathrm{H}$ & 6.01969000 & 3.30951900 & -1.07548000 \\
\hline $\mathrm{H}$ & 9.35766600 & 1.17850800 & 0.67367800 \\
\hline $\mathrm{H}$ & 8.05747900 & 0.88855400 & 1.83964500 \\
\hline $\mathrm{H}$ & 8.59298200 & -0.42094900 & 0.77322200 \\
\hline $\mathrm{C}$ & 2.77175000 & -4.66182300 & 0.35506800 \\
\hline $\mathrm{C}$ & 5.04819900 & -4.48053000 & -1.72032700 \\
\hline $\mathrm{C}$ & 5.71165800 & -4.53471400 & 1.30741600 \\
\hline $\mathrm{H}$ & 2.40479600 & -4.36272300 & 1.34176500 \\
\hline $\mathrm{H}$ & 2.03957000 & -4.34186900 & -0.39315800 \\
\hline $\mathrm{H}$ & 2.80359700 & -5.75538800 & 0.33474100 \\
\hline $\mathrm{H}$ & 6.01804000 & -4.03042800 & -1.94156200 \\
\hline $\mathrm{H}$ & 5.14343600 & -5.56861100 & -1.78940400 \\
\hline $\mathrm{H}$ & 4.33612300 & -4.15461700 & -2.48436500 \\
\hline $\mathrm{H}$ & 5.81860900 & -5.62378600 & 1.29315600 \\
\hline $\mathrm{H}$ & 6.68861900 & -4.08603100 & 1.11593100 \\
\hline $\mathrm{H}$ & 5.38905900 & -4.23947000 & 2.31008500 \\
\hline $\mathrm{C}$ & -1.63425200 & 5.25283400 & -1.98124200 \\
\hline $\mathrm{H}$ & -1.76758200 & 6.33811400 & -1.93794900 \\
\hline $\mathrm{H}$ & -0.56495000 & 5.03312000 & -1.99083500 \\
\hline $\mathrm{H}$ & -2.06690700 & 4.89932300 & -2.92192000 \\
\hline $\mathrm{C}$ & -1.70211400 & 5.03673900 & 1.11585100 \\
\hline $\mathrm{H}$ & -2.12380200 & 4.48823400 & 1.96366900 \\
\hline $\mathrm{H}$ & -0.61944700 & 4.89139000 & 1.09717200 \\
\hline $\mathrm{H}$ & -1.90837500 & 6.10085000 & 1.26309200 \\
\hline $\mathrm{C}$ & -4.30213100 & 4.72754800 & -0.51274800 \\
\hline $\mathrm{H}$ & -4.77568500 & 4.34342300 & -1.42143200 \\
\hline $\mathrm{H}$ & -4.79678100 & 4.26871800 & 0.34857200 \\
\hline $\mathrm{H}$ & -4.49969700 & 5.80287500 & -0.47016500 \\
\hline $\mathrm{C}$ & 1.68057100 & 1.21494800 & -2.78442300 \\
\hline $\mathrm{H}$ & 1.77432600 & 1.27117300 & -3.87394800 \\
\hline $\mathrm{H}$ & 1.59069600 & 2.22982100 & -2.39324200 \\
\hline $\mathrm{H}$ & 2.59354400 & 0.77175200 & -2.38530500 \\
\hline $\mathrm{C}$ & 0.54816200 & -1.64518000 & -2.44145300 \\
\hline $\mathrm{H}$ & 0.87823600 & -1.89894600 & -3.45375900 \\
\hline $\mathrm{H}$ & 1.35903100 & -1.91515200 & -1.76098500 \\
\hline $\mathrm{H}$ & -0.32281300 & -2.26400600 & -2.21257800 \\
\hline $\mathrm{C}$ & -1.23778600 & 0.63957600 & -3.54321900 \\
\hline $\mathrm{H}$ & -0.95537400 & 0.36393100 & -4.56398400 \\
\hline $\mathrm{H}$ & -2.16638600 & 0.12127600 & -3.29601600 \\
\hline $\mathrm{H}$ & -1.43521300 & 1.71434400 & -3.53067300 \\
\hline $\mathrm{H}$ & -3.79961300 & -1.77555700 & -0.20637900 \\
\hline $\mathrm{Si}$ & 7.13845700 & 1.14587400 & -0.46756800 \\
\hline $\mathrm{Si}$ & 4.48985800 & -3.95445800 & -0.00083500 \\
\hline $\mathrm{Si}$ & -2.44144200 & 4.40958200 & -0.50583300 \\
\hline $\mathrm{Si}$ & 0.15908800 & 0.19583500 & -2.36452400 \\
\hline
\end{tabular}




\section{References:}

(1) Keylor, M. H.; Matsuura, B. S.; Stephenson, C. R. J. Chemistry and Biology of Resveratrol-Derived Natural Products. Chem. Rev. 2015, 115 (17), 8976-9027. https://doi.org/10.1021/cr500689b.

(2) Sako, M.; Hosokawa, H.; Ito, T.; Iinuma, M. Regioselective Oxidative Coupling of 4-Hydroxystilbenes: Synthesis of Resveratrol and $\varepsilon$-Viniferin (E)-Dehydrodimers. J. Org. Chem. 2004, 69 (7), 2598-2600. https://doi.org/10.1021/jo035791c.

(3) Romero, K. J.; Galliher, M. S.; Raycroft, M. A. R.; Chauvin, J.-P. R.; Bosque, I.; Pratt, D. A.; Stephenson, C. R. J. Electrochemical Dimerization of Phenylpropenoids and the Surprising Antioxidant Activity of the Resultant Quinone Methide Dimers. Angew. Chem. Int. Ed. 2018, 57 (52), 17125-17129. https://doi.org/10.1002/anie.201810870.

(4) Keylor, M. H.; Matsuura, B. S.; Griesser, M.; Chauvin, J.-P. R.; Harding, R. A.; Kirillova, M. S.; Zhu, X.; Fischer, O. J.; Pratt, D. A.; Stephenson, C. R. J. Synthesis of Resveratrol Tetramers via a Stereoconvergent Radical Equilibrium. Science 2016, 354 (6317), 1260-1265. https://doi.org/10.1126/science.aaj1597.

(5) Bowler, J. M.; Hervert, K. L.; Kearley, M. L.; Miller, B. G. Small-Molecule Allosteric Activation of Human Glucokinase in the Absence of Glucose. ACS Med. Chem. Lett. 2013, 4 (7), 580-584. https://doi.org/10.1021/ml400061x.

(6) Xiong, X.; Tan, F.; Yeung, Y.-Y. Zwitterionic-Salt-Catalyzed Site-Selective Monobromination of Arenes. Org. Lett. 2017, 19 (16), 4243-4246. https://doi.org/10.1021/acs.orglett.7b01899.

(7) Oshima, Y.; Kamijou, A.; Moritani, H.; Namao, K.; Ohizumi, Y. Vitisin A and Cis-Vitisin A, Strongly Hepatotoxic Plant Oligostilbenes from Vitis Coignetiae (Vitaceae). J. Org. Chem. 1993, 58 (4), 850-853. https://doi.org/10.1021/jo00056a016.

(8) Ohshima, Y.; Shinoda, K.; Takaya, Y.; Ohta, T.; Niwa, M.; Hisamichi, K.; Takeshita, M. Vitisins D and E, Novel Oligostilbenes from Vitis Coignetilae Stem Barks. HETEROCYCLES 1997, 46 (1), 169. https://doi.org/10.3987/COM-97-S62.

(9) Lv, H.; Zhou, W.; Wang, X.; Wang, Z.; Suo, Y.; Wang, H. Extraction and Separation of Vitisin D, Ampelopsin B and Cis-Vitisin A from Iris Lactea Pall. Var. Chinensis (Fisch.) Koidz by Alkaline Extraction-Acid Precipitation and High-Speed Counter-Current Chromatography. J. Chromatogr. Sci. 2016, 54 (5), 744-751.

https://doi.org/10.1093/chromsci/bmv249. 